\title{
Discovery of Novel Small-Molecule Antiangiogenesis Agents to Treat Diabetic Retinopathy
}

Donghwa Kim, ${ }^{\text {a, }}{ }^{\dagger}$ Sang Won Choi, ${ }^{\text {a, }}{ }^{\dagger}$ Jihee Cho, ${ }^{a}$ Jae-Hui Been, ${ }^{a}$ Kyoungsun Choi, ${ }^{a}$ Wenzhe Jiang, ${ }^{\mathrm{a}}$ Jaeho Han, ${ }^{\mathrm{a}}$ Jedo Oh, ${ }^{\mathrm{b}}$ Changmin Park, ${ }^{\mathrm{b}}$ Soongyu Choi, ${ }^{\mathrm{b}}$ Songyi Seo, ${ }^{\mathrm{c}}$ Koung Li Kim, ${ }^{\mathrm{c}}$ Wonhee Suh, ${ }^{\mathrm{c}}$ Sang Kook Lee ${ }^{\mathrm{a}, *}$ and Sanghee Kim ${ }^{\mathrm{a}, *}$

${ }^{a}$ College of Pharmacy, Seoul National University, Seoul 08826, Korea

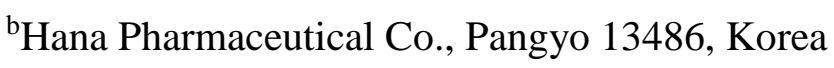

cDepartment of Global Innovative Drug, Graduate School of Chung-Ang University, College of Pharmacy, Chung-Ang University, Seoul 06974, Korea

$\dagger$ equally contributed

\section{Contents}

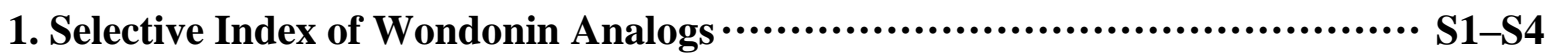

2. IC 50 Graphs for Tube Formation and Cell Viability of $31 \cdots \ldots \ldots \ldots . . . . . . . . . . . . . . .55$

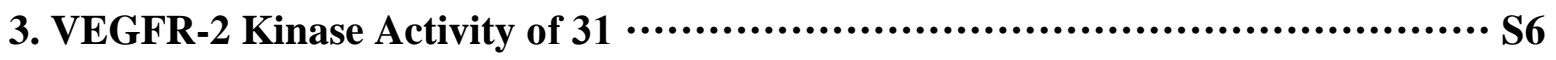

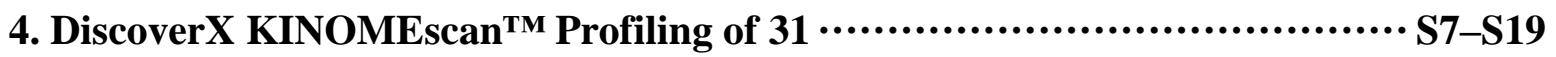

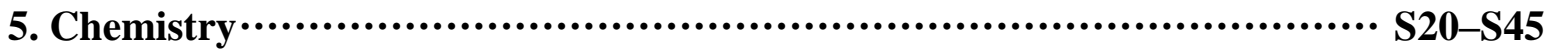

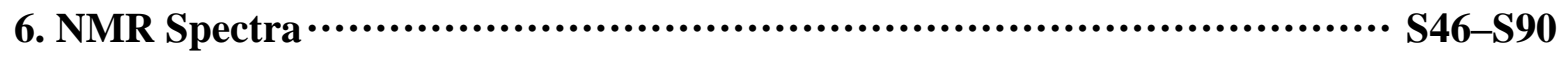

7. HRMS Analysis of Final Target Compounds

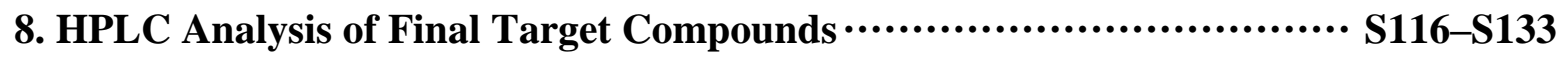

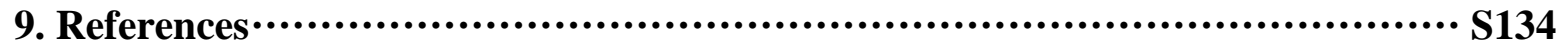




\section{Selective Index of Wondonin Analogs}
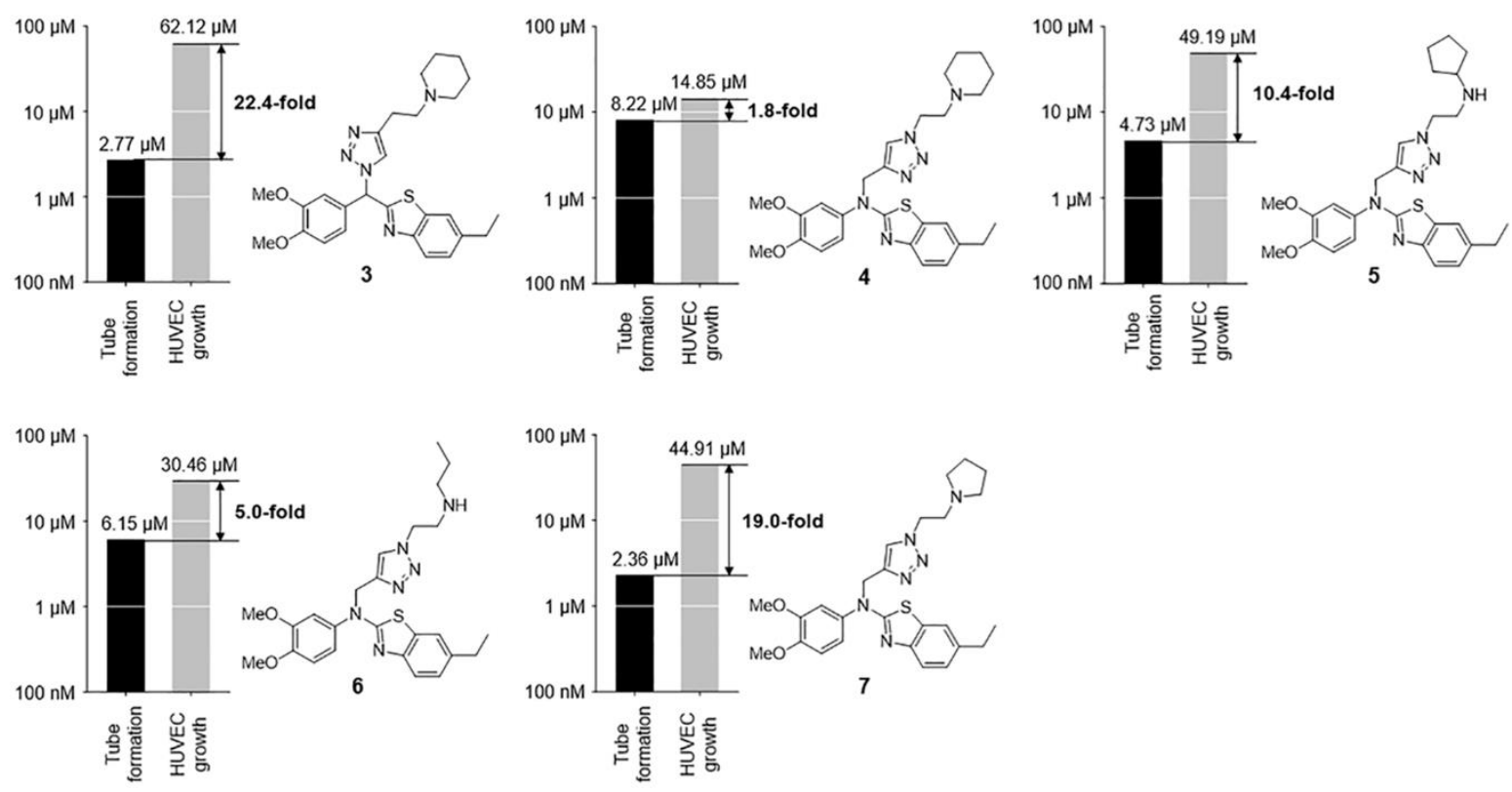

Figure S1. Chemical structures of wondonin analogs 4-7 (scaffold I) and graphs representing the $\mathrm{IC}_{50}$ value of their effects on VEGF-induced tube formation and cell viability of HUVECs. The values represent the average $\mathrm{IC}_{50}$ in duplicate experiments for tube formation and triplicate experiments for cell viability. The fold change was calculated as the ratio of the $\mathrm{IC}_{50}$ values of cell viability and tube formation. 

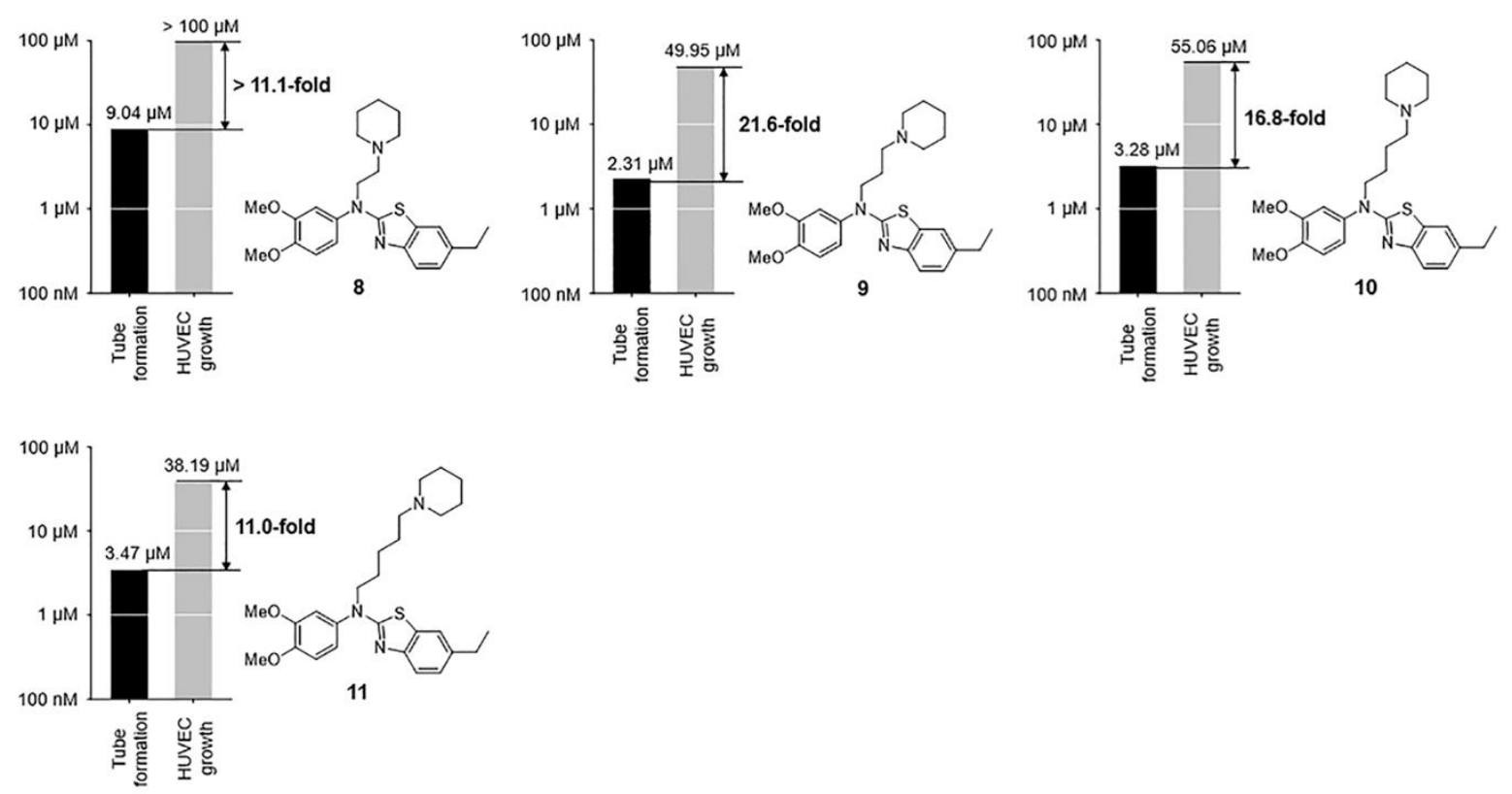

Figure S2. Chemical structures of wondonin analogs 8-11 (scaffold II-A) and graphs representing the $\mathrm{IC}_{50}$ value of their effects on VEGF-induced tube formation and cell viability of HUVECs. The values represent the average $\mathrm{IC}_{50}$ in duplicate experiments for tube formation and triplicate experiments for cell viability. The fold change was calculated as the ratio of the $\mathrm{IC}_{50}$ values of cell viability and tube formation.
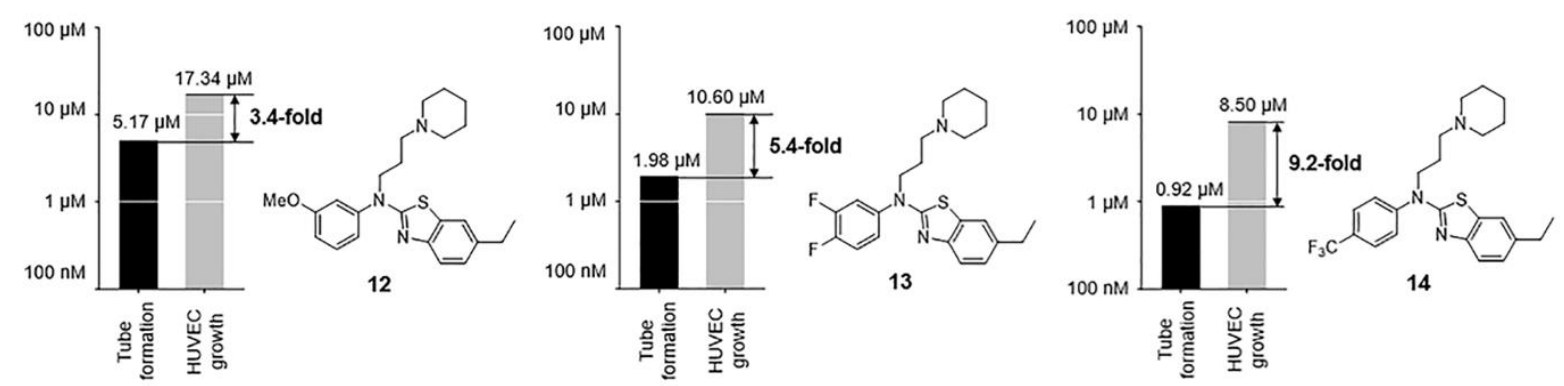

Figure S3. Chemical structures of wondonin analogs 12-14 (scaffold II-B) and graphs representing the $\mathrm{IC}_{50}$ value of their effects on VEGF-induced tube formation and cell viability of HUVECs. The values represent the average $\mathrm{IC}_{50}$ in duplicate experiments for tube formation and triplicate experiments for cell viability. The fold change was calculated as the ratio of the $\mathrm{IC}_{50}$ values of cell viability and tube formation. 

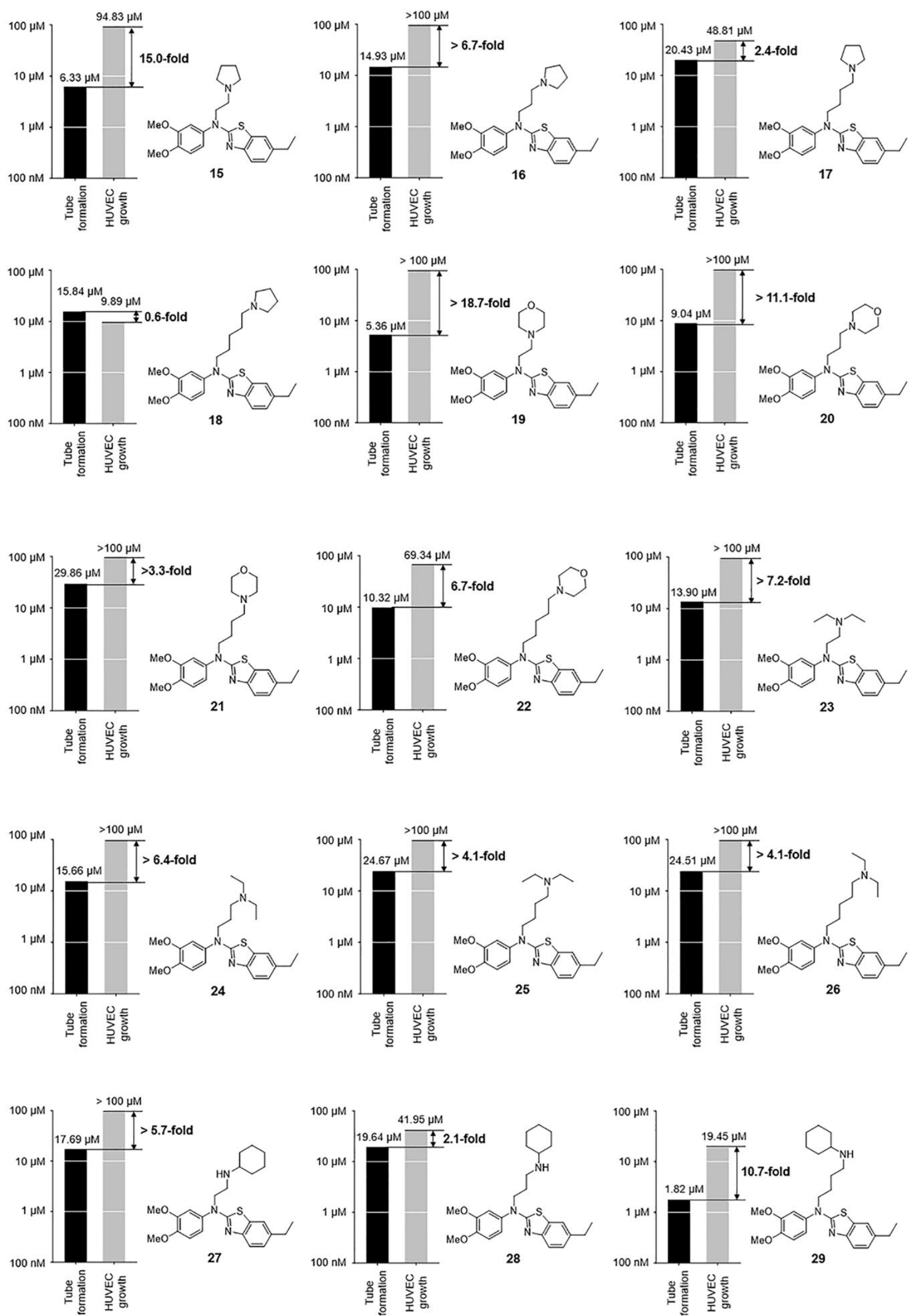

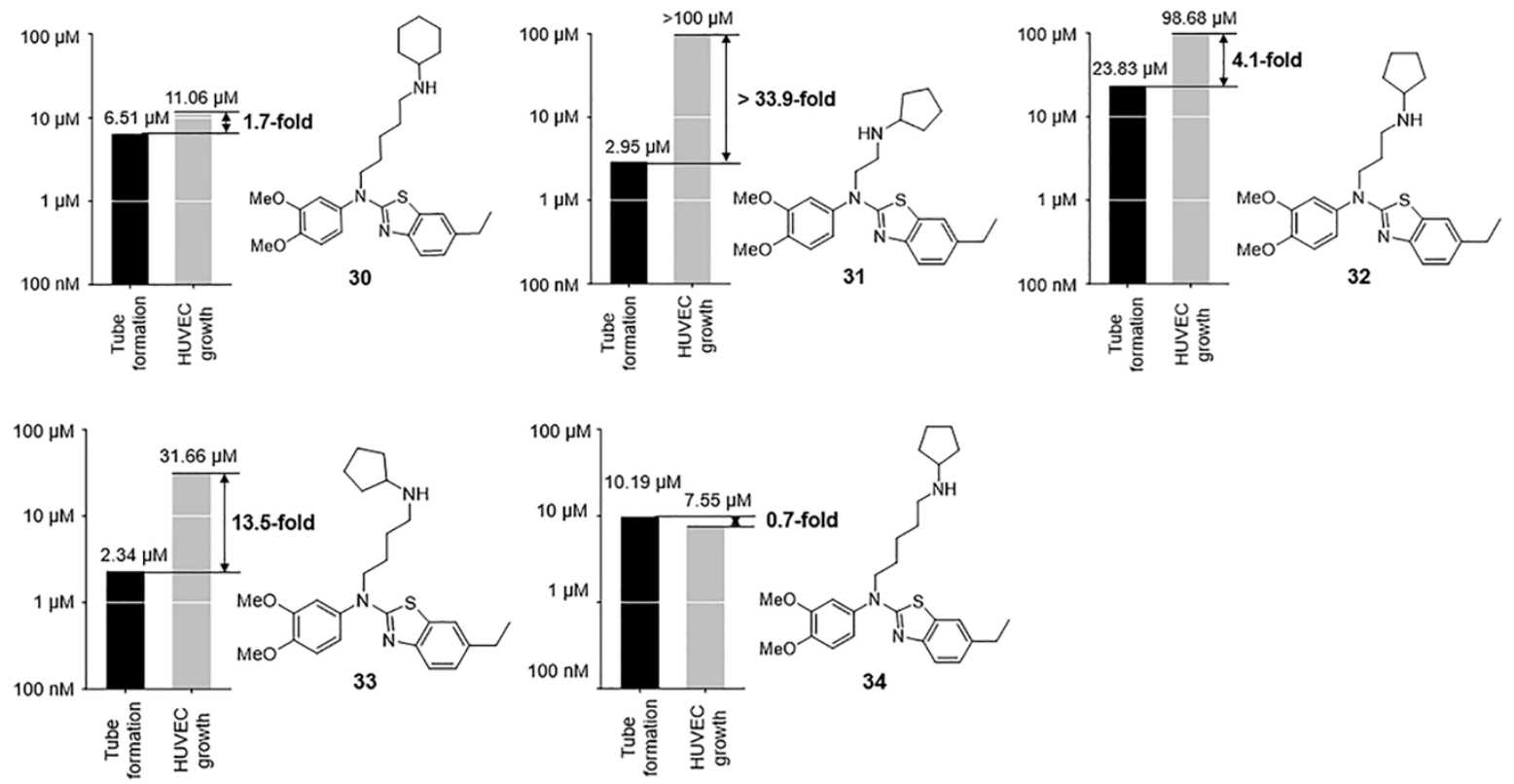

Figure S4. Chemical structures of wondonin analogs 15-34 (scaffold II-C) and graphs representing the $\mathrm{IC}_{50}$ value of their effects on VEGF-induced tube formation and cell viability of HUVECs. The values represent the average $\mathrm{IC}_{50}$ in duplicate experiments for tube formation and triplicate experiments for cell viability. The fold change was calculated as the ratio of the $\mathrm{IC}_{50}$ values of cell viability and tube formation. 


\section{IC $_{50}$ Graphs for Tube Formation and Cell Viability of $\mathbf{3 1}$}

A

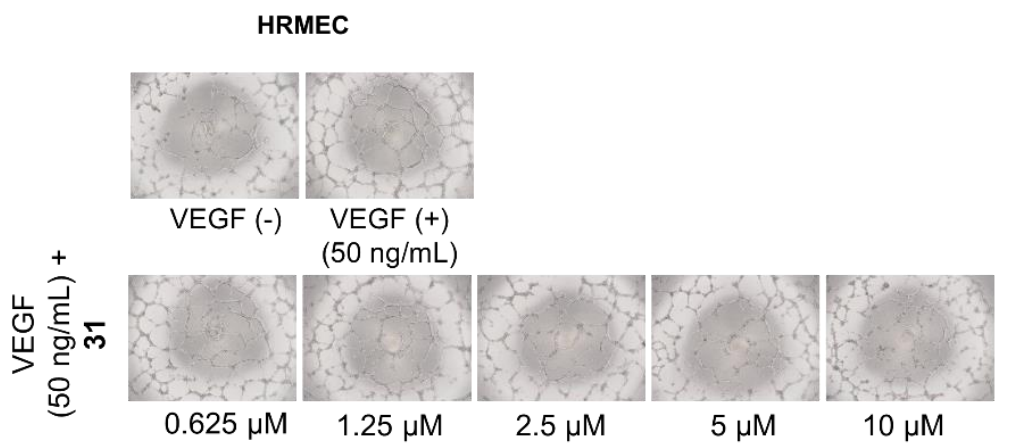

B
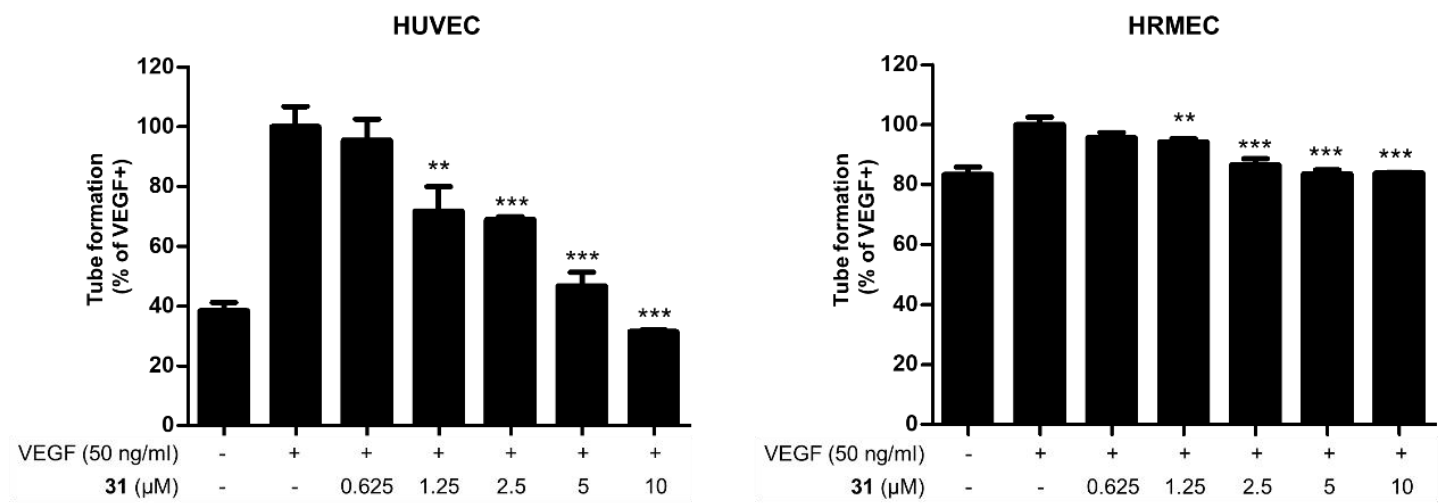

C
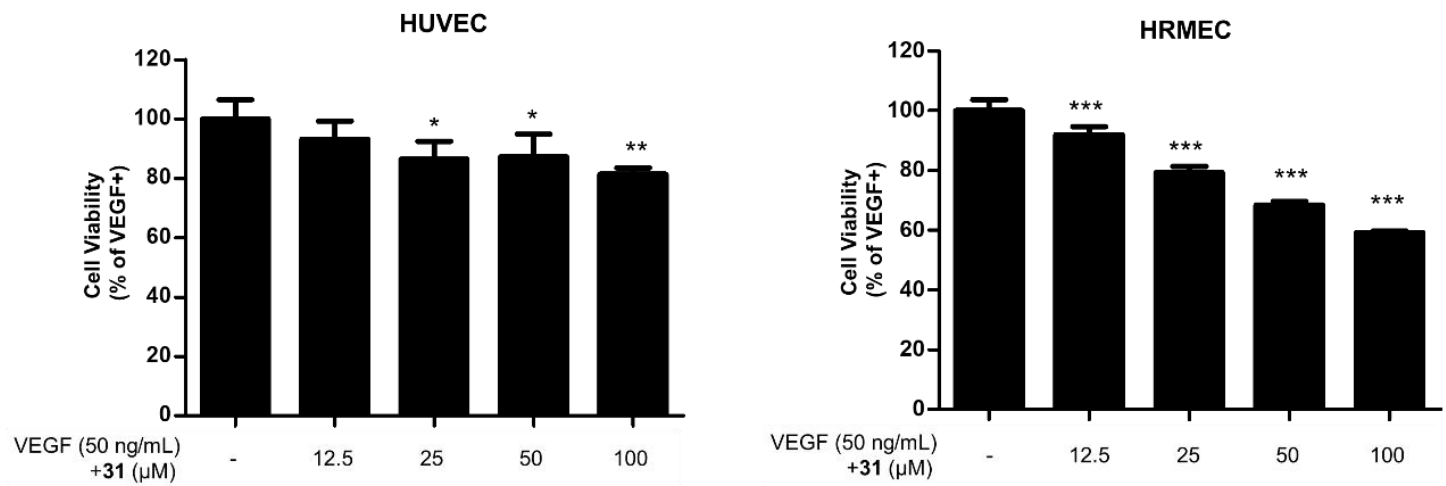

Figure S5. The graphs for tube formation and cell viability of 31. (A) HRMEC cells were seeded in Matrigel-coated 96-well plates and treated with VEGF $(50 \mathrm{ng} / \mathrm{ml})$ in the presence or absence of various concentration of 31. After incubation for 4-8 h, the capillary structures were photographed. (B) Images were quantified using Angiogenesis Analyzer in ImageJ and normalized to values of VEGF (+) control. (C) The cytotoxicity of $\mathbf{3 1}$ was measured after $24 \mathrm{~h}$ treatment for indicated concentrations in the presence of VEGF $(50 \mathrm{ng} / \mathrm{mL})$. Data are presented as the mean fold changes \pm SD of three independent experiments. $* P<0.05, * * P<0.01, * * * P<0.005$ by $t$-test. 


\section{VEGFR-2 Kinase Activity of 31}

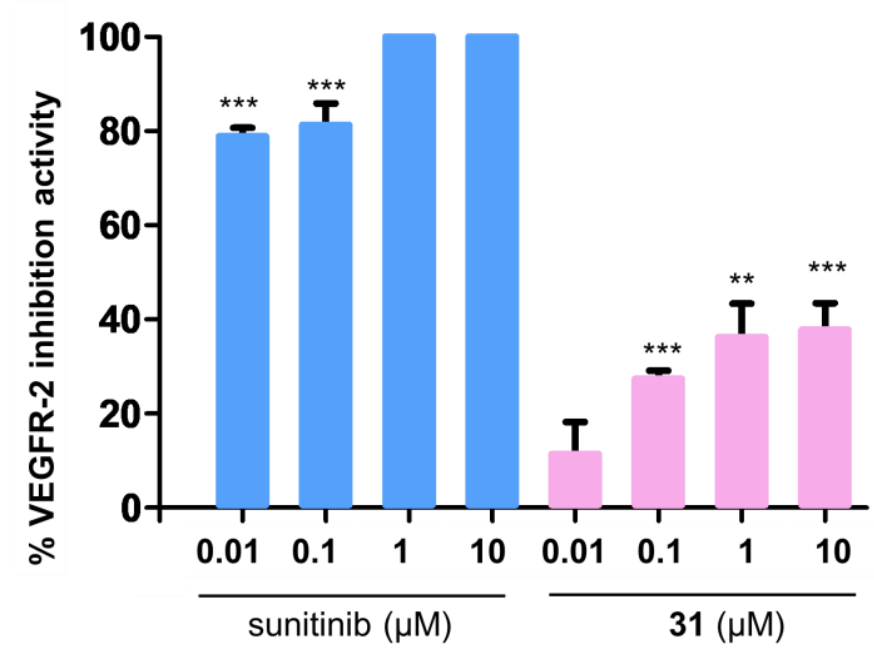

Figure S6. VEGFR-2 kinase activity of 31. VEGFR-2 tyrosine kinase activity of $\mathbf{3 1}$ was determined using Kinase-Glo MAX luminescence kinase assay. Inhibition activity was calculated using following equation: $100-[100 \times$ (test compound signal - blank signal) / (negative control signal - blank signal )]. Data are presented as the mean fold changes \pm SD of three independent experiments. Statistical significance was determined by ANOVA using Dunnett's test (two-sided). $* P<0.05$, $* * P<0.01$, $* * * P<0.005$. 


\section{DiscoverX KINOMEscan ${ }^{\mathrm{TM}}$ Profiling of 31}

Table S1. DiscoverX KINOMEscan ${ }^{\mathrm{TM}}$ Profiling of 31

\begin{tabular}{|c|c|c|}
\hline DiscoveRx Gene Symbol & Entrez Gene Symbol & Percent Control \\
\hline AAK1 & AAK1 & 98 \\
\hline ABL1(E255K)-phosphorylated & ABL1 & 100 \\
\hline ABL1(F317I)-nonphosphorylated & ABL1 & 100 \\
\hline ABL1(F317I)-phosphorylated & ABL1 & 98 \\
\hline ABL1(F317L)-nonphosphorylated & ABL1 & 100 \\
\hline ABL1(F317L)-phosphorylated & ABL1 & 100 \\
\hline ABL1(H396P)-nonphosphorylated & ABL1 & 96 \\
\hline ABL1(H396P)-phosphorylated & ABL1 & 100 \\
\hline ABL1(M351T)-phosphorylated & ABL1 & 100 \\
\hline ABL1(Q252H)-nonphosphorylated & ABL1 & 100 \\
\hline ABL1(Q252H)-phosphorylated & ABL1 & 100 \\
\hline ABL1(T315I)-nonphosphorylated & ABL1 & 100 \\
\hline ABL1(T315I)-phosphorylated & ABL1 & 100 \\
\hline ABL1(Y253F)-phosphorylated & ABL1 & 100 \\
\hline ABL1-nonphosphorylated & ABL1 & 100 \\
\hline ABL1-phosphorylated & ABL1 & 100 \\
\hline ABL2 & ABL2 & 89 \\
\hline ACVR1 & ACVR1 & 100 \\
\hline ACVR1B & ACVR1B & 87 \\
\hline ACVR2A & ACVR2A & 100 \\
\hline ACVR2B & ACVR2B & 99 \\
\hline ACVRL1 & ACVRL1 & 100 \\
\hline ADCK3 & $\mathrm{CABC} 1$ & 97 \\
\hline ADCK4 & ADCK4 & 100 \\
\hline AKT1 & AKT1 & 100 \\
\hline AKT2 & AKT2 & 100 \\
\hline AKT3 & AKT3 & 88 \\
\hline ALK & ALK & 99 \\
\hline $\operatorname{ALK}(\mathrm{C} 1156 \mathrm{Y})$ & ALK & 100 \\
\hline ALK(L1196M) & ALK & 100 \\
\hline AMPK-alpha1 & PRKAA1 & 99 \\
\hline AMPK-alpha2 & PRKAA2 & 96 \\
\hline ANKK1 & ANKK1 & 97 \\
\hline ARK5 & NUAK1 & 100 \\
\hline
\end{tabular}




\begin{tabular}{|c|c|c|}
\hline ASK1 & MAP3K5 & 100 \\
\hline ASK2 & MAP3K6 & 100 \\
\hline AURKA & AURKA & 100 \\
\hline AURKB & AURKB & 100 \\
\hline AURKC & AURKC & 92 \\
\hline AXL & AXL & 76 \\
\hline BIKE & BMP2K & 95 \\
\hline BLK & BLK & 89 \\
\hline BMPR1A & BMPR1A & 91 \\
\hline BMPR1B & BMPR1B & 100 \\
\hline BMPR2 & BMPR2 & 100 \\
\hline BMX & BMX & 91 \\
\hline BRAF & BRAF & 100 \\
\hline BRAF(V600E) & BRAF & 100 \\
\hline BRK & PTK6 & 100 \\
\hline BRSK1 & BRSK1 & 86 \\
\hline BRSK2 & BRSK2 & 100 \\
\hline BTK & BTK & 97 \\
\hline BUB1 & BUB1 & 100 \\
\hline CAMK1 & CAMK1 & 71 \\
\hline CAMK1B & PNCK & 88 \\
\hline CAMK1D & CAMK1D & 100 \\
\hline CAMK1G & CAMK1G & 100 \\
\hline CAMK2A & CAMK2A & 91 \\
\hline CAMK2B & CAMK2B & 95 \\
\hline CAMK2D & CAMK2D & 99 \\
\hline CAMK2G & CAMK2G & 99 \\
\hline CAMK4 & CAMK4 & 100 \\
\hline CAMKK1 & CAMKK1 & 99 \\
\hline CAMKK2 & CAMKK2 & 98 \\
\hline CASK & CASK & 100 \\
\hline CDC2L1 & CDK11B & 100 \\
\hline CDC2L2 & CDC2L2 & 100 \\
\hline CDC2L5 & CDK13 & 96 \\
\hline CDK11 & CDK19 & 100 \\
\hline CDK2 & $\mathrm{CDK} 2$ & 93 \\
\hline CDK3 & CDK3 & 100 \\
\hline CDK4 & $\mathrm{CDK} 4$ & 100 \\
\hline
\end{tabular}




\begin{tabular}{|c|c|c|}
\hline CDK4-cyclinD1 & CDK4 & 99 \\
\hline CDK4-cyclinD3 & CDK4 & 100 \\
\hline CDK5 & CDK5 & 93 \\
\hline CDK7 & CDK7 & 100 \\
\hline CDK8 & CDK8 & 100 \\
\hline CDK9 & CDK9 & 100 \\
\hline CDKL1 & CDKL1 & 100 \\
\hline CDKL2 & CDKL2 & 100 \\
\hline CDKL3 & CDKL3 & 100 \\
\hline CDKL5 & CDKL5 & 100 \\
\hline CHEK1 & CHEK1 & 99 \\
\hline CHEK2 & CHEK2 & 100 \\
\hline CIT & CIT & 100 \\
\hline CLK1 & CLK1 & 100 \\
\hline CLK2 & CLK2 & 100 \\
\hline CLK3 & CLK3 & 100 \\
\hline CLK4 & CLK4 & 89 \\
\hline CSF1R & CSF1R & 100 \\
\hline CSF1R-autoinhibited & CSF1R & 100 \\
\hline CSK & CSK & 100 \\
\hline CSNK1A1 & CSNK1A1 & 100 \\
\hline CSNK1A1L & CSNK1A1L & 95 \\
\hline CSNK1D & CSNK1D & 99 \\
\hline CSNK1E & CSNK1E & 97 \\
\hline CSNK1G1 & CSNK1G1 & 100 \\
\hline CSNK1G2 & CSNK1G2 & 91 \\
\hline CSNK1G3 & CSNK1G3 & 100 \\
\hline CSNK2A1 & CSNK2A1 & 100 \\
\hline CSNK2A2 & CSNK2A2 & 100 \\
\hline CTK & MATK & 100 \\
\hline DAPK1 & DAPK1 & 98 \\
\hline DAPK2 & DAPK2 & 96 \\
\hline DAPK3 & DAPK3 & 96 \\
\hline DCAMKL1 & DCLK1 & 93 \\
\hline DCAMKL2 & DCLK2 & 100 \\
\hline DCAMKL3 & DCLK3 & 97 \\
\hline DDR1 & DDR1 & 98 \\
\hline DDR2 & DDR2 & 81 \\
\hline
\end{tabular}




\begin{tabular}{|c|c|c|}
\hline DLK & MAP3K12 & 90 \\
\hline DMPK & DMPK & 100 \\
\hline DMPK2 & CDC42BPG & 96 \\
\hline DRAK1 & STK17A & 94 \\
\hline DRAK2 & STK17B & 92 \\
\hline DYRK1A & DYRK1A & 100 \\
\hline DYRK1B & DYRK1B & 100 \\
\hline DYRK2 & DYRK2 & 100 \\
\hline EGFR & EGFR & 98 \\
\hline EGFR(E746-A750del) & EGFR & 85 \\
\hline EGFR(G719C) & EGFR & 89 \\
\hline EGFR(G719S) & EGFR & 86 \\
\hline EGFR(L747-E749del, A750P) & EGFR & 96 \\
\hline EGFR(L747-S752del, P753S) & EGFR & 96 \\
\hline EGFR(L747-T751del,Sins) & EGFR & 86 \\
\hline EGFR(L858R) & EGFR & 100 \\
\hline EGFR(L858R,T790M) & EGFR & 100 \\
\hline EGFR(L861Q) & EGFR & 87 \\
\hline EGFR(S752-I759del) & EGFR & 88 \\
\hline EGFR(T790M) & EGFR & 98 \\
\hline EIF2AK1 & EIF2AK1 & 100 \\
\hline EPHA1 & EPHA1 & 100 \\
\hline EPHA2 & EPHA2 & 91 \\
\hline EPHA3 & EPHA3 & 88 \\
\hline EPHA4 & EPHA4 & 91 \\
\hline EPHA5 & EPHA5 & 91 \\
\hline EPHA6 & EPHA6 & 98 \\
\hline EPHA7 & EPHA7 & 89 \\
\hline EPHA8 & EPHA8 & 96 \\
\hline EPHB1 & EPHB1 & 94 \\
\hline EPHB2 & EPHB2 & 100 \\
\hline EPHB3 & EPHB3 & 100 \\
\hline EPHB4 & EPHB4 & 100 \\
\hline EPHB6 & EPHB6 & 100 \\
\hline ERBB2 & ERBB2 & 100 \\
\hline ERBB3 & ERBB3 & 90 \\
\hline ERBB4 & ERBB4 & 93 \\
\hline ERK1 & MAPK3 & 92 \\
\hline
\end{tabular}




\begin{tabular}{|c|c|c|}
\hline ERK2 & MAPK1 & 95 \\
\hline ERK3 & MAPK6 & 100 \\
\hline ERK4 & MAPK4 & 100 \\
\hline ERK5 & MAPK7 & 100 \\
\hline ERK8 & MAPK15 & 100 \\
\hline ERN1 & ERN1 & 100 \\
\hline FAK & PTK2 & 98 \\
\hline FER & FER & 97 \\
\hline FES & FES & 100 \\
\hline FGFR1 & FGFR1 & 100 \\
\hline FGFR2 & FGFR2 & 98 \\
\hline FGFR3 & FGFR3 & 92 \\
\hline FGFR3(G697C) & FGFR3 & 100 \\
\hline FGFR4 & FGFR4 & 96 \\
\hline FGR & FGR & 100 \\
\hline FLT1 & FLT1 & 96 \\
\hline FLT3 & FLT3 & 83 \\
\hline FLT3(D835H) & FLT3 & 100 \\
\hline FLT3(D835V) & FLT3 & 100 \\
\hline FLT3(D835Y) & FLT3 & 96 \\
\hline FLT3(ITD) & FLT3 & 99 \\
\hline FLT3(ITD,D835V) & FLT3 & 100 \\
\hline FLT3(ITD,F691L) & FLT3 & 94 \\
\hline FLT3(K663Q) & FLT3 & 100 \\
\hline FLT3(N841I) & FLT3 & 100 \\
\hline FLT3(R834Q) & FLT3 & 86 \\
\hline FLT3-autoinhibited & FLT3 & 100 \\
\hline FLT4 & FLT4 & 100 \\
\hline FRK & FRK & 100 \\
\hline FYN & FYN & 100 \\
\hline GAK & GAK & 100 \\
\hline GCN2(Kin.Dom.2,S808G) & EIF2AK4 & 100 \\
\hline GRK1 & GRK1 & 100 \\
\hline GRK2 & ADRBK1 & 76 \\
\hline GRK3 & ADRBK2 & 92 \\
\hline GRK4 & GRK4 & 100 \\
\hline GRK7 & GRK7 & 100 \\
\hline GSK3A & GSK3A & 100 \\
\hline
\end{tabular}




\begin{tabular}{|c|c|c|}
\hline GSK3B & GSK3B & 100 \\
\hline HASPIN & GSG2 & 91 \\
\hline HCK & $\mathrm{HCK}$ & 100 \\
\hline HIPK1 & HIPK1 & 100 \\
\hline HIPK2 & HIPK2 & 96 \\
\hline HIPK3 & HIPK3 & 100 \\
\hline HIPK4 & HIPK4 & 100 \\
\hline HPK1 & MAP4K1 & 89 \\
\hline HUNK & HUNK & 63 \\
\hline ICK & ICK & 93 \\
\hline IGF1R & IGF1R & 95 \\
\hline IKK-alpha & CHUK & 100 \\
\hline IKK-beta & IKBKB & 100 \\
\hline IKK-epsilon & IKBKE & 100 \\
\hline INSR & INSR & 97 \\
\hline INSRR & INSRR & 100 \\
\hline IRAK1 & IRAK1 & 100 \\
\hline IRAK3 & IRAK3 & 84 \\
\hline IRAK4 & IRAK4 & 94 \\
\hline ITK & ITK & 90 \\
\hline JAK1(JH1domain-catalytic) & JAK1 & 99 \\
\hline JAK1(JH2domain-pseudokinase) & JAK1 & 97 \\
\hline JAK2(JH1domain-catalytic) & JAK2 & 100 \\
\hline JAK3(JH1domain-catalytic) & JAK3 & 94 \\
\hline JNK1 & MAPK8 & 100 \\
\hline JNK2 & MAPK9 & 100 \\
\hline JNK3 & MAPK10 & 100 \\
\hline KIT & KIT & 98 \\
\hline KIT(A829P) & KIT & 100 \\
\hline KIT(D816H) & KIT & 100 \\
\hline KIT(D816V) & KIT & 98 \\
\hline KIT(L576P) & KIT & 97 \\
\hline KIT(V559D) & KIT & 97 \\
\hline KIT(V559D,T670I) & KIT & 97 \\
\hline KIT(V559D,V654A) & KIT & 95 \\
\hline KIT-autoinhibited & KIT & 100 \\
\hline LATS1 & LATS1 & 96 \\
\hline LATS2 & LATS2 & 100 \\
\hline
\end{tabular}




\begin{tabular}{|c|c|c|}
\hline LCK & LCK & 99 \\
\hline LIMK1 & LIMK1 & 100 \\
\hline LIMK2 & LIMK2 & 99 \\
\hline LKB1 & STK11 & 94 \\
\hline LOK & STK10 & 100 \\
\hline LRRK2 & LRRK2 & 88 \\
\hline LRRK2(G2019S) & LRRK2 & 86 \\
\hline LTK & LTK & 96 \\
\hline LYN & LYN & 100 \\
\hline LZK & MAP3K13 & 87 \\
\hline MAK & MAK & 96 \\
\hline MAP3K1 & MAP3K1 & 100 \\
\hline MAP3K15 & MAP3K15 & 100 \\
\hline MAP3K2 & MAP3K2 & 100 \\
\hline MAP3K3 & MAP3K3 & 100 \\
\hline MAP3K4 & MAP3K4 & 100 \\
\hline MAP4K2 & MAP4K2 & 100 \\
\hline MAP4K3 & MAP4K3 & 99 \\
\hline MAP4K4 & MAP4K4 & 100 \\
\hline MAP4K5 & MAP4K5 & 100 \\
\hline MAPKAPK2 & MAPKAPK2 & 100 \\
\hline MAPKAPK5 & MAPKAPK5 & 100 \\
\hline MARK1 & MARK1 & 100 \\
\hline MARK2 & MARK2 & 89 \\
\hline MARK3 & MARK3 & 94 \\
\hline MARK4 & MARK4 & 100 \\
\hline MAST1 & MAST1 & 100 \\
\hline MEK1 & MAP2K1 & 100 \\
\hline MEK2 & MAP2K2 & 100 \\
\hline MEK3 & MAP2K3 & 100 \\
\hline MEK4 & MAP2K4 & 100 \\
\hline MEK5 & MAP2K5 & 100 \\
\hline MEK6 & MAP2K6 & 100 \\
\hline MELK & MELK & 100 \\
\hline MERTK & MERTK & 100 \\
\hline MET & MET & 93 \\
\hline MET(M1250T) & MET & 100 \\
\hline MET(Y1235D) & MET & 100 \\
\hline
\end{tabular}




\begin{tabular}{|c|c|c|}
\hline MINK & MINK1 & 97 \\
\hline MKK7 & MAP2K7 & 100 \\
\hline MKNK1 & MKNK1 & 100 \\
\hline MKNK2 & MKNK2 & 100 \\
\hline MLCK & MYLK3 & 94 \\
\hline MLK1 & MAP3K9 & 95 \\
\hline MLK2 & MAP3K10 & 100 \\
\hline MLK3 & MAP3K11 & 100 \\
\hline MRCKA & CDC42BPA & 100 \\
\hline MRCKB & CDC42BPB & 100 \\
\hline MST1 & STK4 & 100 \\
\hline MST1R & MST1R & 99 \\
\hline MST2 & STK3 & 100 \\
\hline MST3 & STK24 & 100 \\
\hline MST4 & MST4 & 100 \\
\hline MTOR & MTOR & 100 \\
\hline MUSK & MUSK & 99 \\
\hline MYLK & MYLK & 100 \\
\hline MYLK2 & MYLK2 & 94 \\
\hline MYLK4 & MYLK4 & 100 \\
\hline MYO3A & MYO3A & 100 \\
\hline MYO3B & MYO3B & 100 \\
\hline NDR1 & STK38 & 100 \\
\hline NDR2 & STK38L & 85 \\
\hline NEK1 & NEK1 & 86 \\
\hline NEK10 & NEK10 & 91 \\
\hline NEK11 & NEK11 & 100 \\
\hline NEK2 & NEK2 & 100 \\
\hline NEK3 & NEK3 & 100 \\
\hline NEK4 & NEK4 & 100 \\
\hline NEK5 & NEK5 & 93 \\
\hline NEK6 & NEK6 & 100 \\
\hline NEK7 & NEK7 & 91 \\
\hline NEK9 & NEK9 & 95 \\
\hline NIK & MAP3K14 & 100 \\
\hline NIM1 & MGC42105 & 100 \\
\hline NLK & NLK & 98 \\
\hline OSR1 & OXSR1 & 96 \\
\hline
\end{tabular}




\begin{tabular}{|c|c|c|}
\hline p38-alpha & MAPK14 & 100 \\
\hline p38-beta & MAPK11 & 100 \\
\hline p38-delta & MAPK13 & 100 \\
\hline p38-gamma & MAPK12 & 100 \\
\hline PAK1 & PAK1 & 95 \\
\hline PAK2 & PAK2 & 83 \\
\hline PAK3 & PAK3 & 95 \\
\hline PAK4 & PAK4 & 95 \\
\hline PAK6 & PAK6 & 100 \\
\hline PAK7 & PAK7 & 93 \\
\hline PCTK1 & CDK16 & 99 \\
\hline PCTK2 & CDK17 & 97 \\
\hline PCTK3 & CDK18 & 100 \\
\hline PDGFRA & PDGFRA & 100 \\
\hline PDGFRB & PDGFRB & 100 \\
\hline PDPK1 & PDPK1 & 100 \\
\hline PFCDPK1(P.falciparum) & CDPK1 & 95 \\
\hline PFPK5(P.falciparum) & MAL13P1.279 & 94 \\
\hline PFTAIRE2 & CDK15 & 93 \\
\hline PFTK1 & CDK14 & 98 \\
\hline PHKG1 & PHKG1 & 87 \\
\hline PHKG2 & PHKG2 & 100 \\
\hline PIK3C2B & PIK3C2B & 99 \\
\hline PIK3C2G & PIK3C2G & 100 \\
\hline PIK3CA & PIK3CA & 100 \\
\hline PIK3CA(C420R) & PIK3CA & 100 \\
\hline PIK3CA(E542K) & PIK3CA & 100 \\
\hline PIK3CA(E545A) & PIK3CA & 100 \\
\hline PIK3CA(E545K) & PIK3CA & 100 \\
\hline PIK3CA(H1047L) & PIK3CA & 100 \\
\hline PIK3CA(H1047Y) & PIK3CA & 98 \\
\hline PIK3CA(I800L) & PIK3CA & 100 \\
\hline PIK3CA(M1043I) & PIK3CA & 100 \\
\hline PIK3CA(Q546K) & PIK3CA & 100 \\
\hline PIK3CB & PIK3CB & 100 \\
\hline PIK3CD & PIK3CD & 93 \\
\hline PIK3CG & PIK3CG & 100 \\
\hline PIK4CB & PI4KB & 100 \\
\hline
\end{tabular}




\begin{tabular}{|c|c|c|}
\hline PIKFYVE & PIKFYVE & 100 \\
\hline PIM1 & PIM1 & 96 \\
\hline PIM2 & PIM2 & 98 \\
\hline PIM3 & PIM3 & 94 \\
\hline PIP5K1A & PIP5K1A & 100 \\
\hline PIP5K1C & PIP5K1C & 100 \\
\hline PIP5K2B & PIP4K2B & 97 \\
\hline PIP5K2C & PIP4K2C & 100 \\
\hline PKAC-alpha & PRKACA & 97 \\
\hline PKAC-beta & PRKACB & 89 \\
\hline PKMYT1 & PKMYT1 & 100 \\
\hline PKN1 & PKN1 & 99 \\
\hline PKN2 & PKN2 & 78 \\
\hline PKNB(M.tuberculosis) & pknB & 95 \\
\hline PLK1 & PLK1 & 100 \\
\hline PLK2 & PLK2 & 100 \\
\hline PLK3 & PLK3 & 100 \\
\hline PLK4 & PLK4 & 100 \\
\hline PRKCD & PRKCD & 100 \\
\hline PRKCE & PRKCE & 100 \\
\hline PRKCH & PRKCH & 94 \\
\hline PRKCI & PRKCI & 100 \\
\hline PRKCQ & PRKCQ & 92 \\
\hline PRKD1 & PRKD1 & 100 \\
\hline PRKD2 & PRKD2 & 100 \\
\hline PRKD3 & PRKD3 & 100 \\
\hline PRKG1 & PRKG1 & 92 \\
\hline PRKG2 & PRKG2 & 100 \\
\hline PRKR & EIF2AK2 & 100 \\
\hline PRKX & PRKX & 88 \\
\hline PRP4 & PRPF4B & 92 \\
\hline PYK2 & PTK2B & 98 \\
\hline QSK & KIAA0999 & 100 \\
\hline RAF1 & RAF1 & 89 \\
\hline RET & RET & 86 \\
\hline RET(M918T) & RET & 98 \\
\hline RET(V804L) & RET & 95 \\
\hline RET(V804M) & RET & 96 \\
\hline
\end{tabular}




\begin{tabular}{|c|c|c|}
\hline RIOK1 & RIOK1 & 98 \\
\hline RIOK2 & RIOK2 & 100 \\
\hline RIOK3 & RIOK3 & 91 \\
\hline RIPK1 & RIPK1 & 100 \\
\hline RIPK2 & RIPK2 & 98 \\
\hline RIPK4 & RIPK4 & 100 \\
\hline RIPK5 & DSTYK & 100 \\
\hline ROCK1 & ROCK1 & 94 \\
\hline ROCK2 & ROCK2 & 100 \\
\hline ROS1 & ROS1 & 100 \\
\hline RPS6KA4(Kin.Dom.1-N-terminal) & RPS6KA4 & 95 \\
\hline RPS6KA4(Kin.Dom.2-C-terminal) & RPS6KA4 & 99 \\
\hline RPS6KA5(Kin.Dom.1-N-terminal) & RPS6KA5 & 100 \\
\hline RPS6KA5(Kin.Dom.2-C-terminal) & RPS6KA5 & 88 \\
\hline RSK1(Kin.Dom.1-N-terminal) & RPS6KA1 & 100 \\
\hline RSK1(Kin.Dom.2-C-terminal) & RPS6KA1 & 99 \\
\hline RSK2(Kin.Dom.1-N-terminal) & RPS6KA3 & 100 \\
\hline RSK2(Kin.Dom.2-C-terminal) & RPS6KA3 & 96 \\
\hline RSK3(Kin.Dom.1-N-terminal) & RPS6KA2 & 98 \\
\hline RSK3(Kin.Dom.2-C-terminal) & RPS6KA2 & 100 \\
\hline RSK4(Kin.Dom.1-N-terminal) & RPS6KA6 & 99 \\
\hline RSK4(Kin.Dom.2-C-terminal) & RPS6KA6 & 99 \\
\hline S6K1 & RPS6KB1 & 100 \\
\hline SBK1 & SBK1 & 92 \\
\hline SGK & SGK1 & 100 \\
\hline SgK110 & SgK110 & 100 \\
\hline SGK2 & SGK2 & 98 \\
\hline SGK3 & SGK3 & 99 \\
\hline SIK & SIK1 & 97 \\
\hline SIK2 & SIK2 & 97 \\
\hline SLK & SLK & 100 \\
\hline SNARK & NUAK2 & 96 \\
\hline SNRK & SNRK & 90 \\
\hline SRC & SRC & 99 \\
\hline SRMS & SRMS & 100 \\
\hline SRPK1 & SRPK1 & 100 \\
\hline SRPK2 & SRPK2 & 100 \\
\hline SRPK3 & SRPK3 & 95 \\
\hline
\end{tabular}




\begin{tabular}{|c|c|c|}
\hline STK16 & STK16 & 100 \\
\hline STK33 & STK33 & 100 \\
\hline STK35 & STK35 & 100 \\
\hline STK36 & STK36 & 100 \\
\hline STK39 & STK39 & 100 \\
\hline SYK & SYK & 100 \\
\hline TAK1 & MAP3K7 & 95 \\
\hline TAOK1 & TAOK1 & 100 \\
\hline TAOK2 & TAOK2 & 100 \\
\hline TAOK3 & TAOK3 & 100 \\
\hline TBK1 & TBK1 & 100 \\
\hline TEC & TEC & 77 \\
\hline TESK1 & TESK1 & 100 \\
\hline TGFBR1 & TGFBR1 & 96 \\
\hline TGFBR2 & TGFBR2 & 97 \\
\hline TIE1 & TIE1 & 100 \\
\hline TIE2 & TEK & 91 \\
\hline TLK1 & TLK1 & 99 \\
\hline TLK2 & TLK2 & 100 \\
\hline TNIK & TNIK & 98 \\
\hline TNK1 & TNK1 & 100 \\
\hline TNK2 & TNK2 & 100 \\
\hline TNNI3K & TNNI3K & 87 \\
\hline TRKA & NTRK1 & 100 \\
\hline TRKB & NTRK2 & 100 \\
\hline TRKC & NTRK3 & 100 \\
\hline TRPM6 & TRPM6 & 86 \\
\hline TSSK1B & TSSK1B & 100 \\
\hline TSSK3 & TSSK3 & 92 \\
\hline TTK & TTK & 93 \\
\hline TXK & TXK & 98 \\
\hline TYK2(JH1domain-catalytic) & TYK2 & 94 \\
\hline TYK2(JH2domain-pseudokinase) & TYK2 & 100 \\
\hline TYRO3 & TYRO3 & 98 \\
\hline ULK1 & ULK1 & 97 \\
\hline ULK2 & ULK2 & 100 \\
\hline ULK3 & ULK3 & 100 \\
\hline VEGFR2 & KDR & 100 \\
\hline
\end{tabular}




\begin{tabular}{|l|l|l|}
\hline VPS34 & PIK3C3 & 92 \\
\hline VRK2 & VRK2 & 97 \\
\hline WEE1 & WEE1 & 98 \\
\hline WEE2 & WEE2 & 99 \\
\hline WNK1 & WNK1 & 99 \\
\hline WNK2 & WNK2 & 94 \\
\hline WNK3 & WNK3 & 94 \\
\hline WNK4 & WNK4 & 82 \\
\hline YANK1 & STK32A & 100 \\
\hline YANK2 & STK32B & 89 \\
\hline YANK3 & STK32C & 100 \\
\hline YES & YES1 & 100 \\
\hline YSK1 & STK25 & 100 \\
\hline YSK4 & MAP3K19 & 100 \\
\hline ZAK & ZAK & 100 \\
\hline ZAP70 & ZAP70 & 98 \\
\hline
\end{tabular}




\section{Chemistry}

General Methods. All the chemicals used were purchased in reagent grade from commercial suppliers, and all the reactions were executed under an inert atmosphere comprising dry nitrogen using distilled dry solvents. The reactions were monitored by thin-layer chromatography (TLC) performed on silica gel $60 \mathrm{~F}_{254}$ TLC plates. The synthesized products were purified by flash column chromatography on silica gel $60(40-63 \mu \mathrm{m}, 230-400$ mesh, Merck). NMR spectra were recorded using a JNMECZ400S/L1 spectrometer (Jeol, Akishima, Tokyo, Japan). ${ }^{1} \mathrm{H}$ NMR (400 MHz) and ${ }^{13} \mathrm{C}$ NMR (100 $\mathrm{MHz}$ ) spectra were obtained in $\delta$ units using residual $\mathrm{CDCl}_{3}\left({ }^{1} \mathrm{H} \mathrm{NMR} \delta=7.26 \mathrm{ppm},{ }^{13} \mathrm{C}\right.$ NMR $\delta=$ $77.16 \mathrm{ppm})$ and $\mathrm{CD}_{2} \mathrm{Cl}_{2}\left({ }^{1} \mathrm{H}\right.$ NMR $\delta=5.32 \mathrm{ppm},{ }^{13} \mathrm{C}$ NMR $\left.\delta=53.84 \mathrm{ppm}\right)$ as the internal standards. IR spectra were obtained using an Agilent 5500a FTIR system (Agilent, USA). High-resolution mass spectra (HRMS) were obtained using fast atom bombardment (FAB) and electrospray ionization (ESI). The purity of all the final target compounds was confirmed to be at least $>95 \%$ pure by analytical HPLC (Agilent 1200 Series; Agilent Technologies, Palo Alto, CA, USA).

\section{(1) Synthesis of Phenylaminothiazolines 35-38}

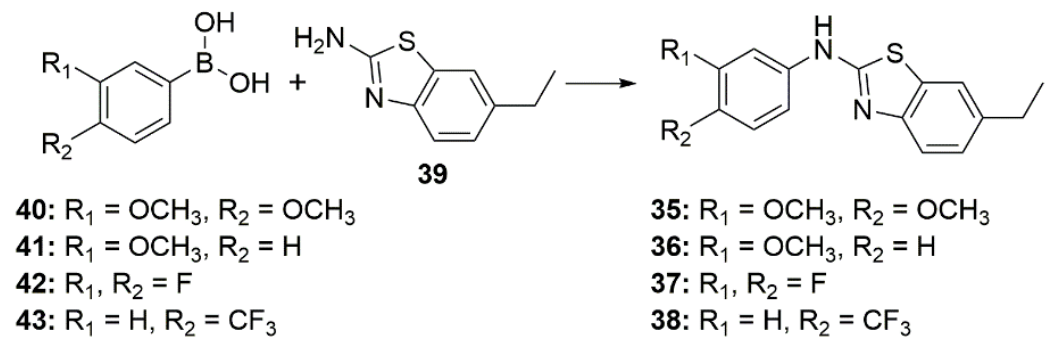

$N$-(3,4-Dimethoxyphenyl)-6-ethylbenzo[d]thiazol-2-amine (35). Compound 35 was synthesized from commercially purchased (3,4-dimethoxyphenyl)boronic acid $\mathbf{4 0}$ and aminothiazoline $\mathbf{3 9} .{ }^{\mathrm{S} 1}$ To a solution of compound 39 (4.00 g, $22.4 \mathrm{mmol})$ in DMSO $(44.9 \mathrm{~mL}, 0.5 \mathrm{M})$ was added compound 40 (6.11 g, $33.6 \mathrm{mmol}), \mathrm{Cu}(\mathrm{OAc})_{2}(1.63 \mathrm{~g}, 8.96 \mathrm{mmol}), 2,2^{\prime}$-bipyridine $(1.40 \mathrm{~g}, 8.96 \mathrm{mmol})$ and $\mathrm{Cs}_{2} \mathrm{CO}_{3}$ (14.6 g, $44.8 \mathrm{mmol})$ at room temperature. The reaction mixture was stirred at $70{ }^{\circ} \mathrm{C}$ for $14 \mathrm{~h}$ and then 
diluted with $\mathrm{H}_{2} \mathrm{O}$ and extracted with EtOAc. The obtained organic layer was dried over $\mathrm{MgSO}_{4}$, filtered and evaporated in vacuo. The crude product was separated by silica gel column chromatography (hexane/EtOAc, 5:1, $\left.\mathrm{R}_{\mathrm{f}}=0.25\right)$ to produce the desired product $35\left(3.73 \mathrm{~g}, 53 \%\right.$, white solid). ${ }^{1} \mathrm{H}$ NMR (400 MHz, $\left.\mathrm{CD}_{2} \mathrm{Cl}_{2}\right) \delta 7.37(\mathrm{~d}, J=2.0 \mathrm{~Hz}, 1 \mathrm{H}), 7.27-7.24(\mathrm{~m}, 1 \mathrm{H}), 7.18(\mathrm{~d}, J=8.3 \mathrm{~Hz}, 1 \mathrm{H}), 6.81$ (brs, $1 \mathrm{H}), 6.75(\mathrm{~d}, J=8.1 \mathrm{~Hz}, 1 \mathrm{H}), 6.68-6.65(\mathrm{~m}, 2 \mathrm{H}), 3.76(\mathrm{~s}, 3 \mathrm{H}), 3.71(\mathrm{~s}, 3 \mathrm{H}), 2.58(\mathrm{q}, J=7.6 \mathrm{~Hz}, 2 \mathrm{H})$, $1.18(\mathrm{t}, J=7.6 \mathrm{~Hz}, 3 \mathrm{H}) ;{ }^{13} \mathrm{C} \mathrm{NMR}\left(100 \mathrm{MHz}, \mathrm{CDCl}_{3}\right) \delta 149.7,148.6,140.3,136.8,135.8,130.8,125.6$, $121.1,119.0,115.3,112.1,111.9,110.7,56.1,56.0,28.0,15.7$; IR (neat) $v_{\max }=3193,2964,2228$, 1496, 1249, 1278, 1136, 1022, 831, $797\left(\mathrm{~cm}^{-1}\right)$; HRMS (FAB): calcd. for $\mathrm{C}_{17} \mathrm{H}_{18} \mathrm{~N}_{2} \mathrm{O}_{2} \mathrm{~S}[\mathrm{M}]^{+}$ 314.1089, found 314.1085.

6-Ethyl- $N$-(3-methoxyphenyl)benzo[d]thiazol-2-amine (36). Compound 36 was synthesized from 39 (500 $\mathrm{mg} ; 2.81 \mathrm{mmol}$ ) following the procedure for the synthesis of 35. Instead of $(3,4-$ dimethoxyphenyl)boronic acid 40, (3-methoxyphenyl)boronic acid 41 (640 mg, $4.21 \mathrm{mmol}$ ) was used, and the reaction mixture was stirred for $14 \mathrm{~h}$ at $70^{\circ} \mathrm{C}$. The crude mixture was separated by silica gel column chromatography (hexane/EtOAc, $\left.5: 1, \mathrm{R}_{\mathrm{f}}=0.3\right)$ to produce the desired product $\mathbf{3 6}(359 \mathrm{mg}, 45 \%$, white solid). ${ }^{1} \mathrm{H}$ NMR (400 MHz, $\left.\mathrm{CDCl}_{3}\right) \delta 7.42(\mathrm{~d}, J=1.8 \mathrm{~Hz}, 1 \mathrm{H}), 7.33-7.31(\mathrm{~m}, 1 \mathrm{H}), 7.26(\mathrm{~d}, J=$ $8.3 \mathrm{~Hz}, 1 \mathrm{H}), 7.17$ (t, $J=8.0 \mathrm{~Hz}, 1 \mathrm{H}), 6.79$ (brs, 1H), 6.72-6.69 (m, 1H), 6.60-6.58 (m, 1H), 6.55 (t, $J$ $=2.1 \mathrm{~Hz}, 1 \mathrm{H}), 3.73(\mathrm{~s}, 3 \mathrm{H}), 2.63(\mathrm{q}, J=7.6 \mathrm{~Hz}, 2 \mathrm{H}), 1.23(\mathrm{t}, J=7.6 \mathrm{~Hz}, 3 \mathrm{H}) ;{ }^{13} \mathrm{C} \mathrm{NMR}(100 \mathrm{MHz}$, $\left.\mathrm{CDCl}_{3}\right) \delta 160.4,140.5,137.6,136.9,136.6,131.6,130.4,119.0,116.7,115.4,112.6,111.9,110.5,55.4$ 28.0, 15.6; IR (neat) $v_{\max }=3171,2963,2229,1584,1494,1244,1030,831,779\left(\mathrm{~cm}^{-1}\right)$; HRMS (ESI): calcd. for $\mathrm{C}_{16} \mathrm{H}_{17} \mathrm{~N}_{2} \mathrm{OS}[\mathrm{M}+\mathrm{H}]^{+} 285.1056$, found 285.1052 .

$N$-(3,4-Difluorophenyl)-6-ethylbenzo[d] thiazol-2-amine (37). Compound 37 was synthesized from 39 (500 $\mathrm{mg}, 2.81 \mathrm{mmol}$ ) following the procedure for the synthesis of 35. Instead of $(3,4-$ dimethoxyphenyl)boronic acid compound 40, (3,4-difluorophenyl)boronic acid compound 42 (665 mg, $4.21 \mathrm{mmol}$ ) was used, and the reaction mixture was stirred for $14 \mathrm{~h}$ at $70{ }^{\circ} \mathrm{C}$. The crude mixture was separated by silica gel column chromatography (hexane/EtOAc, $7: 1, \mathrm{R}_{\mathrm{f}}=0.2$ ) to produce the desired product 37 (506 mg, 62\%, white solid). ${ }^{1} \mathrm{H}$ NMR (400 MHz, $\left.\mathrm{CDCl}_{3}\right) \delta 7.39$ (d, $\left.J=2.0 \mathrm{~Hz}, 1 \mathrm{H}\right), 7.36-$ 
$7.34(\mathrm{~m}, 1 \mathrm{H}), 7.28(\mathrm{~d}, J=8.3 \mathrm{~Hz}, 1 \mathrm{H}), 7.07$ (td, $J=9.9,8.4 \mathrm{~Hz}, 1 \mathrm{H}), 6.84-6.75(\mathrm{~m}, 3 \mathrm{H}), 2.64$ (q, $J=$ $7.6 \mathrm{~Hz}, 2 \mathrm{H}), 1.23(\mathrm{t}, J=7.6 \mathrm{~Hz}, 3 \mathrm{H}) ;{ }^{13} \mathrm{C} \mathrm{NMR}\left(100 \mathrm{MHz}, \mathrm{CDCl}_{3}\right) \delta 151.32(\mathrm{dd}, J=13.0,158.6 \mathrm{~Hz}$, 1C), $148.83(\mathrm{dd}, J=12.8,155.5 \mathrm{~Hz}, 1 \mathrm{C}), 140.9,137.4,136.8,132.0,131.70$ (q, $J=3.2 \mathrm{~Hz}, 1 \mathrm{C}), 123.06$ $(\mathrm{q}, J=3.2 \mathrm{~Hz}, 1 \mathrm{C}), 118.38(\mathrm{~d}, J=17.9 \mathrm{~Hz}, 1 \mathrm{C}), 116.33$ (d, $J=14.4 \mathrm{~Hz}, 1 \mathrm{C}), 116.1,115.6,110.3,28.0$, 15.6; IR (neat) $v_{\max }=3185,8966,2228,1599,1496,1394,1271,1196,900,770\left(\mathrm{~cm}^{-1}\right)$; HRMS (ESI): calcd. for $\mathrm{C}_{15} \mathrm{H}_{13} \mathrm{~F}_{2} \mathrm{~N}_{2} \mathrm{~S}[\mathrm{M}+\mathrm{H}]^{+}$291.0762, found 291.0759 .

6-Ethyl- $N$-(4-(trifluoromethyl)phenyl)benzo $[\boldsymbol{d}]$ thiazol-2-amine $\quad \mathbf{( 3 8 )}$. Compound $\quad \mathbf{3 8}$ was synthesized from 39 (400 $\mathrm{mg}, 2.24 \mathrm{mmol})$ following the procedure for the synthesis of $\mathbf{3 5}$. Instead of (3,4-dimethoxyphenyl)boronic acid 40, (4-(trifluoromethyl)phenyl)boronic acid 43 (639 mg, 3.37 mmol) was used, and the reaction mixture was stirred for $14 \mathrm{~h}$ at $70^{\circ} \mathrm{C}$. The crude mixture was separated by silica gel column chromatography (hexane/EtOAc, 5:1, $\mathrm{R}_{\mathrm{f}}=0.3$ ) to produce the desired product 38 (333 mg, 46\%, ivory solid). ${ }^{1} \mathrm{H}$ NMR (400 MHz, $\left.\mathrm{CDCl}_{3}\right) \delta 7.49$ (d, $\left.J=8.4 \mathrm{~Hz}, 2 \mathrm{H}\right), 7.42-7.37$ (m, 2H), $7.30(\mathrm{~d}, J=8.3 \mathrm{~Hz}, 1 \mathrm{H}), 7.06(\mathrm{~d}, J=8.3 \mathrm{~Hz}, 2 \mathrm{H}), 2.65(\mathrm{q}, J=7.6 \mathrm{~Hz}, 2 \mathrm{H}), 1.24(\mathrm{t}, J=7.6 \mathrm{~Hz}, 3 \mathrm{H}) ;{ }^{13} \mathrm{C}$ $\operatorname{NMR}\left(100 \mathrm{MHz}, \mathrm{CDCl}_{3}\right) \delta 140.9,140.7,137.9,137.1,132.2,128.51$ (q, $\left.J=29.3 \mathrm{~Hz}, 1 \mathrm{C}\right), 126.33$ (q, $J$ $=3.6 \mathrm{~Hz}, 2 \mathrm{C}), 126.1(2 \mathrm{C}), 123.97(\mathrm{q}, J=267.1 \mathrm{~Hz}, 1 \mathrm{C}), 115.7,115.2,110.3,28.0,15.6$; IR (neat) $v_{\text {ma }}$ $x=3202,2970,2227,1603,1496,1324,1288,1107,825\left(\mathrm{~cm}^{-1}\right)$; HRMS (ESI): calcd. for $\mathrm{C}_{16} \mathrm{H}_{14} \mathrm{~F}_{3} \mathrm{~N}_{2} \mathrm{~S}[\mathrm{M}+\mathrm{H}]^{+}$323.0824, found 323.0815.

\section{(2) Synthesis of 1,2,3-Triazole Analogs 4-7}

\section{A. Synthetic Procedures for the Preparation of 44, 46 and 47}

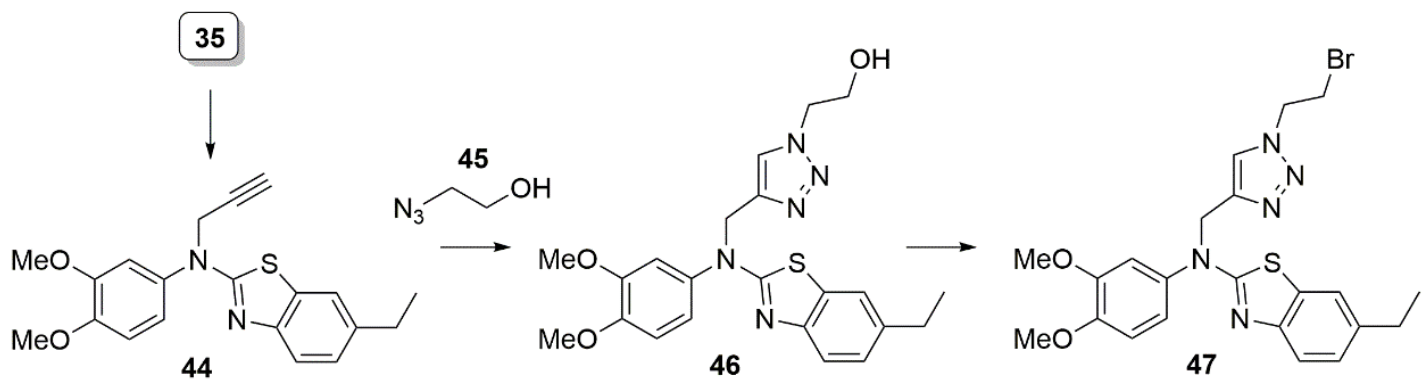


$N$-(3,4-Dimethoxyphenyl)-6-ethyl- $N$-(prop-2-yn-1-yl)benzo[d] thiazol-2-amine (44). To a solution of compound 35 (700 mg, $2.23 \mathrm{mmol})$ in dry DMF $(7.42 \mathrm{~mL}, 0.3 \mathrm{M})$ was added $\mathrm{K}_{2} \mathrm{CO}_{3}(616 \mathrm{mg}, 4.46$ $\mathrm{mmol}$ ), and the mixture was stirred at $0{ }^{\circ} \mathrm{C}$ for $10 \mathrm{~min}$. To this reaction mixture was added propargyl bromide ( $80 \%$ in toluene, $298 \mu \mathrm{L}, 2.67 \mathrm{mmol}$ ), and then the mixture was allowed to warm to room temperature slowly and stirred for $16 \mathrm{~h}$. The reaction mixture was diluted with $\mathrm{H}_{2} \mathrm{O}$ and extracted with EtOAc. The organic layer was dried over $\mathrm{MgSO}_{4}$, filtered and evaporated in vacuo. The crude product was separated by silica gel column chromatography (hexane/EtOAc, 5:1, $\mathrm{R}_{\mathrm{f}}=0.3$ ) to produce the desired product 44 (723 mg, 92\%, white solid). ${ }^{1} \mathrm{H}$ NMR (400 MHz, $\left.\mathrm{CDCl}_{3}\right) \delta 7.38(\mathrm{~d}, J=8.0 \mathrm{~Hz}, 1 \mathrm{H})$, 7.10-7.07 (m, 1H), 7.03-7.00 (m, 1H), $6.98(\mathrm{~d}, J=2.1 \mathrm{~Hz}, 1 \mathrm{H}), 6.90(\mathrm{~d}, J=8.3 \mathrm{~Hz}, 1 \mathrm{H}), 6.73(\mathrm{~d}, J=$ $1.9 \mathrm{~Hz}, 1 \mathrm{H}), 4.23(\mathrm{~d}, J=2.5 \mathrm{~Hz}, 2 \mathrm{H}), 3.91(\mathrm{~s}, 3 \mathrm{H}), 3.85(\mathrm{~s}, 3 \mathrm{H}), 2.52-2.46(\mathrm{~m}, 3 \mathrm{H}), 1.10(\mathrm{t}, J=7.6 \mathrm{~Hz}$ $3 \mathrm{H}) ;{ }^{13} \mathrm{C} \mathrm{NMR}\left(100 \mathrm{MHz}, \mathrm{CDCl}_{3}\right) \delta 150.1,149.7,145.9,136.4,134.1,128.4,127.7,126.9,126.5,121.8$, $117.1,115.0,112.0,76.4,76.0,56.2,56.1,43.7,28.6,15.4$; IR (neat) $v_{\max }=3296,2966,2220,158$ 4, 1502, 1248, 1233, 1134, 1019, $850\left(\mathrm{~cm}^{-1}\right)$; HRMS (FAB): calcd. for $\mathrm{C}_{20} \mathrm{H}_{21} \mathrm{~N}_{2} \mathrm{O}_{2} \mathrm{~S}[\mathrm{M}+\mathrm{H}]^{+}$ 353.1324, found 353.1317 .

\section{2-(4-(((3,4-Dimethoxyphenyl)(6-ethylbenzo[d] thiazol-2-yl)amino)methyl)-1H-1,2,3-triazol-1-}

yl)ethan-1-ol (46). Compound 46 was synthesized from compound 44 and azide $45 .{ }^{\text {S2 }}$ To a solution of compound $44(723 \mathrm{mg}, 2.05 \mathrm{mmol})$ in $\mathrm{EtOH} / t-\mathrm{BuOH} / \mathrm{H}_{2} \mathrm{O}(1: 1: 1,6.83 \mathrm{~mL}, 0.3 \mathrm{M})$ was added compound 45 (214 mg, $2.46 \mathrm{mmol}), \mathrm{CuSO}_{4} \cdot 5 \mathrm{H}_{2} \mathrm{O}(25.6 \mathrm{mg}, 0.10 \mathrm{mmol})$ and sodium ascorbate (40.6 $\mathrm{mg}, 0.21 \mathrm{mmol})$ at room temperature. The reaction mixture was stirred at $60{ }^{\circ} \mathrm{C}$ for $20 \mathrm{~min}$ and then diluted with $\mathrm{H}_{2} \mathrm{O}$ and extracted with EtOAc. The organic layer was dried over $\mathrm{MgSO}_{4}$, filtered and evaporated in vacuo. The crude product was separated by silica gel column chromatography $\left(\mathrm{CH}_{2} \mathrm{Cl}_{2} / \mathrm{MeOH}, 50: 1, \mathrm{R}_{\mathrm{f}}=0.25\right)$ to produce the desired product $46\left(788 \mathrm{mg}, 87 \%\right.$, white solid). ${ }^{1} \mathrm{H}$ NMR (400 MHz, $\left.\mathrm{CDCl}_{3}\right) \delta 7.90(\mathrm{~s}, 1 \mathrm{H}), 7.17(\mathrm{~d}, J=8.1 \mathrm{~Hz}, 1 \mathrm{H}), 7.09-7.07(\mathrm{~m}, 1 \mathrm{H}), 6.99(\mathrm{~d}, J=1.8$ $\mathrm{Hz}, 1 \mathrm{H}), 6.96-6.94(\mathrm{~m}, 1 \mathrm{H}), 6.89(\mathrm{~d}, J=8.3 \mathrm{~Hz}, 1 \mathrm{H}), 6.72-6.71(\mathrm{~m}, 1 \mathrm{H}), 4.71(\mathrm{~s}, 2 \mathrm{H}), 4.49(\mathrm{t}, J=4.9$ Hz, 2H), 4.01 (t, $J=4.9 \mathrm{~Hz}, 2 \mathrm{H}$ ), $3.91(\mathrm{~s}, 3 \mathrm{H}), 3.84(\mathrm{~s}, 3 \mathrm{H}), 2.78$ (brs, $1 \mathrm{H}), 2.46$ (q, $J=7.6 \mathrm{~Hz}, 2 \mathrm{H})$, $1.07(\mathrm{t}, J=7.6 \mathrm{~Hz}, 3 \mathrm{H}) ;{ }^{13} \mathrm{C} \mathrm{NMR}\left(100 \mathrm{MHz}, \mathrm{CDCl}_{3}\right) \delta 150.0,149.7,145.6,141.5,136.3,134.6,128.6$, 127.6, 126.7, 126.6, 124.8, 122.1, 117.1, 115.4, 112.0, 61.1, 56.2, 56.1, 52.9, 49.1, 28.5, 15.4; IR (nea 
t) $v_{\max }=3468,3150,2928,2220,1584,1501,1249,1228,1130,1019,840\left(\mathrm{~cm}^{-1}\right)$; HRMS (FAB): calcd. for $\mathrm{C}_{22} \mathrm{H}_{26} \mathrm{~N}_{5} \mathrm{O}_{3} \mathrm{~S}[\mathrm{M}+\mathrm{H}]^{+} 440.1756$, found 440.1759 .

\section{$N$-((1-(2-Bromoethyl)-1H-1,2,3-triazol-4-yl)methyl)- $N$-(3,4-dimethoxyphenyl)-6}

ethylbenzo[d]thiazol-2-amine (47). To a solution of compound 46 (98.4 mg, $0.22 \mathrm{mmol})$ in dry $\mathrm{CH}_{2} \mathrm{Cl}_{2}$ (1.10 mL, $0.2 \mathrm{M})$ was added $\mathrm{PPh}_{3}(115 \mathrm{mg}, 0.44 \mathrm{mmol})$ and $\mathrm{CBr}_{4}(146 \mathrm{mg}, 0.44 \mathrm{mmol})$, and the mixture was stirred at room temperature for $2 \mathrm{~h}$. The reaction mixture was diluted with $\mathrm{H}_{2} \mathrm{O}$ and extracted with EtOAc. The organic layer was dried over $\mathrm{MgSO}_{4}$, filtered and evaporated in vacuo. The crude product was separated by silica gel column chromatography (hexane/EtOAc, 1:1.2, $\mathrm{R}_{\mathrm{f}}=0.25$ ) to produce the desired product $47\left(97.7 \mathrm{mg}, 88 \%\right.$, white solid). ${ }^{1} \mathrm{H}$ NMR (400 MHz, $\left.\mathrm{CDCl}_{3}\right) \delta 7.86(\mathrm{~s}, 1 \mathrm{H}), 7.14(\mathrm{~d}, J$ $=8.0 \mathrm{~Hz}, 1 \mathrm{H}), 7.09-7.07(\mathrm{~m}, 1 \mathrm{H}), 6.99(\mathrm{~d}, J=2.1 \mathrm{~Hz}, 1 \mathrm{H}), 6.95-6.92(\mathrm{~m}, 1 \mathrm{H}), 6.89(\mathrm{~d}, J=8.4 \mathrm{~Hz}, 1 \mathrm{H})$, $6.73(\mathrm{~d}, J=1.8 \mathrm{~Hz}, 1 \mathrm{H}), 4.77-4.67(\mathrm{~m}, 4 \mathrm{H}), 3.92-3.90(\mathrm{~m}, 3.5 \mathrm{H}), 3.84(\mathrm{~s}, 3 \mathrm{H}), 3.75(\mathrm{t}, J=6.1 \mathrm{~Hz}$, $1.5 \mathrm{H}), 2.46(\mathrm{q}, J=7.6 \mathrm{~Hz}, 2 \mathrm{H}), 1.07(\mathrm{t}, J=7.6 \mathrm{~Hz}, 3 \mathrm{H}) ;{ }^{13} \mathrm{C} \mathrm{NMR}\left(100 \mathrm{MHz}, \mathrm{CDCl}_{3}\right) \delta 149.9,149.7$, $145.5,141.7,141.6,136.2,134.6,128.7,127.5,126.7,126.6,124.5,124.3,122.3,117.0,115.3,112.0$, $56.2,56.1,51.9,51.8,49.1,42.5,29.5,28.5,15.4$; IR (neat) $v_{\max }=3144,2966,2216,1581,1501$, 1228, 1251, 1132, 1021, $840\left(\mathrm{~cm}^{-1}\right)$; HRMS (FAB): calcd. for $\mathrm{C}_{22} \mathrm{H}_{25} \mathrm{BrN}_{5} \mathrm{O}_{2} \mathrm{~S}[\mathrm{M}+\mathrm{H}]^{+} 502.0912$, found 502.0920 .

\section{B. Synthetic Procedures for the Preparation of 4-7}

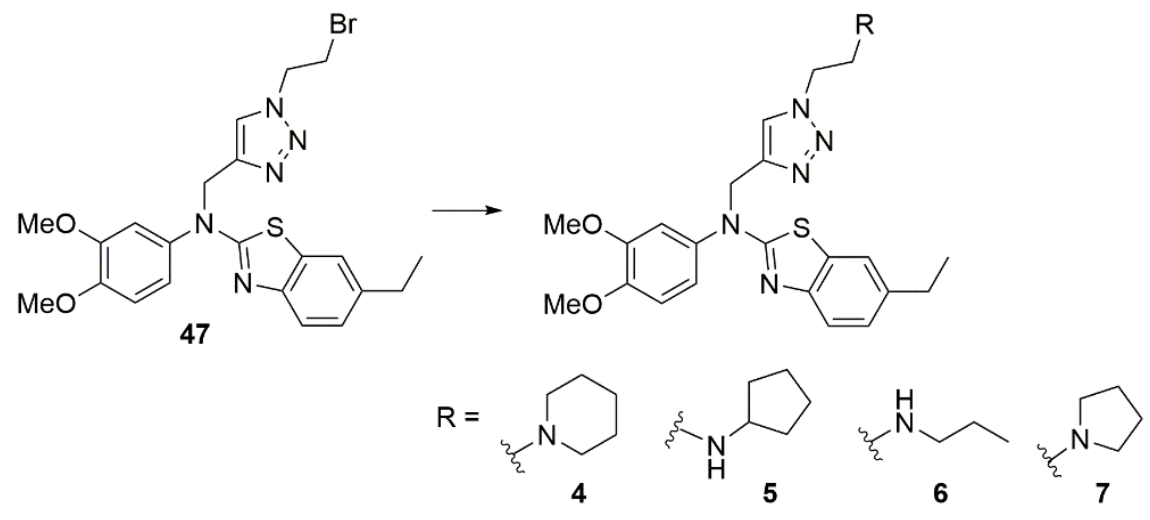


$N$-(3,4-Dimethoxyphenyl)-6-ethyl- $N$-((1-(2-(piperidin-1-yl)ethyl)-1H-1,2,3-triazol-4-yl)methyl)be nzo $[\boldsymbol{d}]$ thiazol-2-amine (4). To a solution of $47(100 \mathrm{mg}, 0.20 \mathrm{mmol})$ in $\mathrm{DMF} / \mathrm{MeCN}(1: 1,2 \mathrm{~mL}, 0$. $1 \mathrm{M})$ was added $\mathrm{K}_{2} \mathrm{CO}_{3}(110 \mathrm{mg}, 0.80 \mathrm{mmol})$ and piperidine $(200 \mu \mathrm{L}, 1.99 \mathrm{mmol})$. The reaction mixture was stirred at $50{ }^{\circ} \mathrm{C}$ for $19 \mathrm{~h}$ and then diluted with $\mathrm{H}_{2} \mathrm{O}$ and extracted with EtOAc. The organic layer was dried over $\mathrm{MgSO}_{4}$, filtered and evaporated in vacuo. The crude product was separated by silica gel column chromatography $\left(\mathrm{CH}_{2} \mathrm{Cl}_{2} / \mathrm{MeOH}, 30: 1, \mathrm{R}_{\mathrm{f}}=0.2\right)$ to produce the desired product $4(96.0 \mathrm{mg}$, 95\%, ivory solid). ${ }^{1} \mathrm{H}$ NMR (400 MHz, $\left.\mathrm{CDCl}_{3}\right) \delta 7.96(\mathrm{~s}, 1 \mathrm{H}), 7.15(\mathrm{~d}, J=8.0 \mathrm{~Hz}, 1 \mathrm{H}), 7.11-7.08$ (m, $1 \mathrm{H}), 7.01(\mathrm{~d}, J=2.0 \mathrm{~Hz}, 1 \mathrm{H}), 6.96-6.93(\mathrm{~m}, 1 \mathrm{H}), 6.90(\mathrm{~d}, J=8.3 \mathrm{~Hz}, 1 \mathrm{H}), 6.74-6.73(\mathrm{~m}, 1 \mathrm{H}), 4.74(\mathrm{~s}$, 2H), $4.46(\mathrm{t}, J=6.2 \mathrm{~Hz}, 2 \mathrm{H}), 3.92(\mathrm{~s}, 3 \mathrm{H}), 3.86(\mathrm{~s}, 3 \mathrm{H}), 2.75(\mathrm{t}, J=6.2 \mathrm{~Hz}, 2 \mathrm{H}), 2.50-2.41(\mathrm{~m}, 6 \mathrm{H})$, $1.60-1.54(\mathrm{~m}, 4 \mathrm{H}), 1.45-1.40(\mathrm{~m}, 2 \mathrm{H}), 1.08(\mathrm{t}, J=7.6 \mathrm{~Hz}, 3 \mathrm{H}) ;{ }^{13} \mathrm{C} \mathrm{NMR}\left(100 \mathrm{MHz}, \mathrm{CDCl}_{3}\right) \delta 150.0$, $149.8,145.5,141.6,136.4,134.9,128.7,127.6,126.7,126.6,124.1,122.4,117.2,115.5,112.0,58.3$, $56.3,56.1,54.7(2 \mathrm{C}), 49.5,48.1,28.6,26.1(2 \mathrm{C}), 24.3,15.4$; IR (neat) $v_{\max }=2933,2214,1584,15$ 03, 1440, 1253, 1230, 1135, 1022, 807, $762\left(\mathrm{~cm}^{-1}\right)$; HRMS (FAB): calcd. for $\mathrm{C}_{27} \mathrm{H}_{35} \mathrm{~N}_{6} \mathrm{O}_{2} \mathrm{~S}$ $[\mathrm{M}+\mathrm{H}]^{+}$507.2542, found 507.2539.

\section{$\mathrm{N}$-((1-(2-(Cyclopentylamino)ethyl)-1H-1,2,3-triazol-4-yl)methyl)- $N$-(3,4-dimethoxyphenyl)-6-eth}

ylbenzo[d]thiazol-2-amine (5). To a solution of $47(100 \mathrm{mg}, 0.20 \mathrm{mmol})$ in DMF/MeCN $(1: 1,2 \mathrm{~mL}$, $0.1 \mathrm{M})$ was added $\mathrm{K}_{2} \mathrm{CO}_{3}(110 \mathrm{mg}, 0.80 \mathrm{mmol})$ and cyclopentylamine $(200 \mu \mathrm{L}, 1.99 \mathrm{mmol})$. The reaction mixture was stirred at $50{ }^{\circ} \mathrm{C}$ for $16 \mathrm{~h}$ and then diluted with $\mathrm{H}_{2} \mathrm{O}$ and extracted with EtOAc. The organic layer was dried over $\mathrm{MgSO}_{4}$, filtered and evaporated in vacuo. The crude product was separated by silica gel column chromatography $\left(\mathrm{CH}_{2} \mathrm{Cl}_{2} / \mathrm{MeOH}, 25: 1, \mathrm{R}_{\mathrm{f}}=0.2\right)$ to produce the desired product 5 (90.0 mg, 89\%, yellow oil). ${ }^{1} \mathrm{H}$ NMR (400 MHz, $\left.\mathrm{CDCl}_{3}\right) \delta 7.86(\mathrm{~s}, 1 \mathrm{H}), 7.16(\mathrm{~d}, J=8.0 \mathrm{~Hz}, 1 \mathrm{H}), 7.09-$ $7.06(\mathrm{~m}, 1 \mathrm{H}), 6.99(\mathrm{~d}, J=2.1 \mathrm{~Hz}, 1 \mathrm{H}), 6.95-6.92(\mathrm{~m}, 1 \mathrm{H}), 6.89(\mathrm{~d}, J=8.3 \mathrm{~Hz}, 1 \mathrm{H}), 6.73-6.72(\mathrm{~m}, 1 \mathrm{H})$, $4.72(\mathrm{~s}, 2 \mathrm{H}), 4.45(\mathrm{t}, J=5.9 \mathrm{~Hz}, 2 \mathrm{H}), 3.90(\mathrm{~s}, 3 \mathrm{H}), 3.84(\mathrm{~s}, 3 \mathrm{H}), 3.09-3.03(\mathrm{~m}, 3 \mathrm{H}), 2.46(\mathrm{q}, J=7.6 \mathrm{~Hz}$, $2 \mathrm{H}), 1.81-1.75(\mathrm{~m}, 2 \mathrm{H}), 1.66-1.58(\mathrm{~m}, 2 \mathrm{H}), 1.52-1.46(\mathrm{~m}, 3 \mathrm{H}), 1.29-1.21(\mathrm{~m}, 2 \mathrm{H}), 1.07$ (t, $J=7.6 \mathrm{~Hz}$, $3 \mathrm{H}) ;{ }^{13} \mathrm{C}$ NMR $\left(100 \mathrm{MHz}, \mathrm{CDCl}_{3}\right) \delta 150.0,149.7,145.5,141.6,136.3,134.8,128.7,127.6,126.62$, $126.56,124.0,122.3,117.1,115.3,112.0,59.6,56.2,56.1,51.1,49.3,47.8,33.2(2 \mathrm{C}), 28.5,24.0(2 \mathrm{C})$, 15.4; IR (neat) $v_{\max }=2954,2852,2214,1584,1503,1398,1253,1230,1022,762\left(\mathrm{~cm}^{-1}\right)$; 
HRMS (FAB): calcd. for $\mathrm{C}_{27} \mathrm{H}_{35} \mathrm{~N}_{6} \mathrm{O}_{2} \mathrm{~S}[\mathrm{M}+\mathrm{H}]^{+}$507.2542, found 507.2552.

\section{$N$-(3,4-Dimethoxyphenyl)-6-ethyl- $N-((1-(2-($ propylamino)ethyl)-1H-1,2,3-triazol-4-yl)methyl)ben}

zo[d]thiazol-2-amine (6). To a solution of $47(100 \mathrm{mg}, 0.20 \mathrm{mmol})$ in DMF/MeCN $(1: 1,2 \mathrm{~mL}, 0.1$ M) was added $\mathrm{K}_{2} \mathrm{CO}_{3}(110 \mathrm{mg}, 0.80 \mathrm{mmol})$ and propylamine $(164 \mu \mathrm{L}, 1.99 \mathrm{mmol})$. The reaction mixture was stirred at $50{ }^{\circ} \mathrm{C}$ for $16 \mathrm{~h}$ and then diluted with $\mathrm{H}_{2} \mathrm{O}$ and extracted with EtOAc. The organic layer was dried over $\mathrm{MgSO}_{4}$, filtered and evaporated in vacuo. The crude product was separated by silica gel column chromatography $\left(\mathrm{CH}_{2} \mathrm{Cl}_{2} / \mathrm{MeOH}, 20: 1, \mathrm{R}_{\mathrm{f}}=0.2\right)$ to produce the desired product $6(70.0 \mathrm{mg}$, $73 \%$, white solid). ${ }^{1} \mathrm{H}$ NMR $\left(400 \mathrm{MHz}, \mathrm{CDCl}_{3}\right) \delta 7.86(\mathrm{~s}, 1 \mathrm{H}), 7.16(\mathrm{~d}, J=8.0 \mathrm{~Hz}, 1 \mathrm{H}), 7.08(\mathrm{dd}, J=$ 8.2, 2.1 Hz, 1H), $6.99(\mathrm{~d}, J=2.0 \mathrm{~Hz}, 1 \mathrm{H}), 6.94(\mathrm{dd}, J=8.0,1.9 \mathrm{~Hz}, 1 \mathrm{H}), 6.88(\mathrm{~d}, J=8.4 \mathrm{~Hz}, 1 \mathrm{H}), 6.72-$ $6.71(\mathrm{~m}, 1 \mathrm{H}), 4.72(\mathrm{~s}, 2 \mathrm{H}), 4.45(\mathrm{t}, J=5.9 \mathrm{~Hz}, 2 \mathrm{H}), 3.90(\mathrm{~s}, 3 \mathrm{H}), 3.84(\mathrm{~s}, 3 \mathrm{H}), 3.09$ (t, $J=5.9 \mathrm{~Hz}, 2 \mathrm{H})$, $2.56(\mathrm{t}, J=7.2 \mathrm{~Hz}, 2 \mathrm{H}), 2.46(\mathrm{q}, J=7.6 \mathrm{~Hz}, 2 \mathrm{H}), 1.49-1.40(\mathrm{~m}, 3 \mathrm{H}), 1.07(\mathrm{t}, J=7.6 \mathrm{~Hz}, 3 \mathrm{H}), 0.87$ (t, $J$ $=7.4 \mathrm{~Hz}, 3 \mathrm{H}) ;{ }^{13} \mathrm{C} \mathrm{NMR}\left(100 \mathrm{MHz}, \mathrm{CDCl}_{3}\right) \delta 150.0,149.7,145.5,141.6,136.3,134.8,128.6,127.6$, $126.63,126.55,124.0,122.3,117.1,115.3,112.0,56.2,56.1,51.4,50.7,49.3,49.0,28.5,23.2,15.4$, 11.8; IR (neat) $v_{\max }=2927,2214,1584,1503,1440,1253,1230,1138,1022,763\left(\mathrm{~cm}^{-1}\right)$; HRMS (ESI): calcd. for $\mathrm{C}_{25} \mathrm{H}_{33} \mathrm{~N}_{6} \mathrm{O}_{2} \mathrm{~S}[\mathrm{M}+\mathrm{H}]^{+} 481.2380$, found 481.2387 .

\section{$N$-(3,4-Dimethoxyphenyl)-6-ethyl- $N$-((1-(2-(pyrrolidin-1-yl)ethyl)-1H-1,2,3-triazol-4-yl)methyl)b} enzo[d]thiazol-2-amine (7). To a solution of $47(100 \mathrm{mg}, 0.20 \mathrm{mmol})$ in $\mathrm{DMF} / \mathrm{MeCN}(1: 1,2 \mathrm{~mL}, 0$. $1 \mathrm{M})$ was added $\mathrm{K}_{2} \mathrm{CO}_{3}(110 \mathrm{mg}, 0.80 \mathrm{mmol})$ and pyrrolidine $(66.5 \mu \mathrm{L}, 0.80 \mathrm{mmol})$. The reaction mixture was stirred at $50{ }^{\circ} \mathrm{C}$ for $19 \mathrm{~h}$ and then diluted with $\mathrm{H}_{2} \mathrm{O}$ and extracted with EtOAc. The organic layer was dried over $\mathrm{MgSO}_{4}$, filtered and evaporated in vacuo. The crude product was separated by silica gel column chromatography $\left(\mathrm{CH}_{2} \mathrm{Cl}_{2} / \mathrm{MeOH}, 25: 1, \mathrm{R}_{\mathrm{f}}=0.2\right)$ to produce the desired product 7 (62.0 mg, 63\%, ivory solid). ${ }^{1} \mathrm{H}$ NMR (400 MHz, $\left.\mathrm{CDCl}_{3}\right) \delta 7.89(\mathrm{~s}, 1 \mathrm{H}), 7.14(\mathrm{~d}, J=8.0 \mathrm{~Hz}, 1 \mathrm{H}), 7.10$ $7.07(\mathrm{~m}, 1 \mathrm{H}), 7.00(\mathrm{~d}, J=2.0 \mathrm{~Hz}, 1 \mathrm{H}), 6.95-6.93(\mathrm{~m}, 1 \mathrm{H}), 6.89(\mathrm{~d}, J=8.3 \mathrm{~Hz}, 1 \mathrm{H}), 6.73-6.72(\mathrm{~m}, 1 \mathrm{H})$, $4.73(\mathrm{~s}, 2 \mathrm{H}), 4.49(\mathrm{t}, J=6.4 \mathrm{~Hz}, 2 \mathrm{H}), 3.91(\mathrm{~s}, 3 \mathrm{H}), 3.85(\mathrm{~s}, 3 \mathrm{H}), 2.95(\mathrm{t}, J=6.4 \mathrm{~Hz}, 2 \mathrm{H}), 2.56-2.52(\mathrm{~m}$, 4H), 2.47 (q, $J=7.6 \mathrm{~Hz}, 2 \mathrm{H}), 1.81-1.74(\mathrm{~m}, 4 \mathrm{H}), 1.08$ (t, $J=7.6 \mathrm{~Hz}, 3 \mathrm{H}) ;{ }^{13} \mathrm{C} \mathrm{NMR}\left(100 \mathrm{MHz}, \mathrm{CDCl}_{3}\right)$ $\delta 150.0,149.8,145.5,141.7,136.3,134.8,128.7,127.6,126.7,126.6,123.9,122.4,117.1,115.5,112.0$, 
56.2, 56.1, 55.5, 54.2 (2C), 49.7, 49.4, 28.5, 23.7 (2C), 15.4; IR (neat) $v_{\max }=2962,2798,2214,15$ 84, 1503, 1398, 1253, 1230, 1137, 1022, $763\left(\mathrm{~cm}^{-1}\right)$; HRMS (FAB): calcd. for $\mathrm{C}_{26} \mathrm{H}_{33} \mathrm{~N}_{6} \mathrm{O}_{2} \mathrm{~S}$ $[\mathrm{M}+\mathrm{H}]^{+}$493.2386, found 493.2382.

\section{(3) Synthesis of Straight-Chain Alkyl Analogs 8-11 and 15-34}

\section{A. Synthetic Procedures for the Preparation of 49-52}

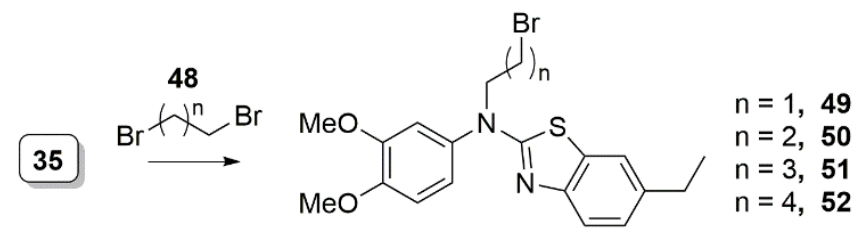

$N$-(2-Bromoethyl)- $N$-(3,4-dimethoxyphenyl)-6-ethylbenzo[d]thiazol-2-amine (49). To a solution of compound 35 (4.22 g, $13.44 \mathrm{mmol})$ in dry DMF (67 mL, 0.2 M) was added $\mathrm{NaH}$ (dry 90\%, $896 \mathrm{mg}$, $33.6 \mathrm{mmol}$ ), and the mixture was stirred at $0{ }^{\circ} \mathrm{C}$ for $10 \mathrm{~min}$. To this reaction mixture was added 1,2dibromo-ethane $(11.6 \mathrm{~mL}, 134 \mathrm{mmol})$, and then the mixture was allowed to warm to room temperature slowly and stirred at $50{ }^{\circ} \mathrm{C}$ for $13 \mathrm{~h}$. Next, the reaction mixture was diluted with $\mathrm{H}_{2} \mathrm{O}$ and extracted with EtOAc. The organic layer was dried over $\mathrm{MgSO}_{4}$, filtered and evaporated in vacuo. The crude product was separated by silica gel column chromatography (hexane/ether, $2: 1, \mathrm{R}_{\mathrm{f}}=0.2$ ) to produce the desired product 49 (4.01 g, 71\%, white solid). ${ }^{1} \mathrm{H}$ NMR (400 MHz, $\left.\mathrm{CDCl}_{3}\right) \delta 7.32(\mathrm{~d}, J=8.0 \mathrm{~Hz}, 1 \mathrm{H}), 7.10$ $7.07(\mathrm{~m}, 1 \mathrm{H}), 7.02-6.98(\mathrm{~m}, 2 \mathrm{H}), 6.90(\mathrm{~d}, J=8.3 \mathrm{~Hz}, 1 \mathrm{H}), 6.73-6.72(\mathrm{~m}, 1 \mathrm{H}), 3.92(\mathrm{~s}, 3 \mathrm{H}), 3.88-3.84$ $(\mathrm{m}, 5 \mathrm{H}), 3.56(\mathrm{t}, J=6.9 \mathrm{~Hz}, 2 \mathrm{H}), 2.49(\mathrm{q}, J=7.6 \mathrm{~Hz}, 2 \mathrm{H}), 1.09(\mathrm{t}, J=7.6 \mathrm{~Hz}, 3 \mathrm{H}) ;{ }^{13} \mathrm{C} \mathrm{NMR}(100 \mathrm{MHz}$, $\left.\mathrm{CDCl}_{3}\right) \delta 150.1,149.8,145.9,136.8,133.7,128.5,127.8,127.3,126.5,121.7,117.2,115.0,112.0,56.2$, $56.1,54.7,28.5,27.6,15.4$; IR (neat) $v_{\max }=2961,2218,1585,1502,1438,1246,1229,1135$, 1018, $847\left(\mathrm{~cm}^{-1}\right)$; HRMS (FAB): calcd. for $\mathrm{C}_{19} \mathrm{H}_{22} \mathrm{BrN}_{2} \mathrm{O}_{2} \mathrm{~S}[\mathrm{M}+\mathrm{H}]^{+} 421.0585$, found 421.0589 .

$N$-(3-Bromopropyl)- $N$-(3,4-dimethoxyphenyl)-6-ethylbenzo[d] thiazol-2-amine (50). To a solution of compound 35 (1.50 g, $4.77 \mathrm{mmol})$ in dry DMF (24 mL, $0.2 \mathrm{M})$ was added $\mathrm{NaH}$ (dry 90\%, $318 \mathrm{mg}$, $11.9 \mathrm{mmol}$ ), and the mixture was stirred at $0{ }^{\circ} \mathrm{C}$ for $10 \mathrm{~min}$. To this reaction mixture was added $1,3-$ 
dibromo-propane $(4.86 \mathrm{~mL}, 47.7 \mathrm{mmol})$, and then the mixture was allowed to warm to room temperature slowly and stirred at $50{ }^{\circ} \mathrm{C}$ for $13 \mathrm{~h}$. Next, the reaction mixture was diluted with $\mathrm{H}_{2} \mathrm{O}$ and extracted with EtOAc. The organic layer was dried over $\mathrm{MgSO}_{4}$, filtered and evaporated in vacuo. The crude product was separated by silica gel column chromatography (hexane/ether, $2: 1, \mathrm{R}_{\mathrm{f}}=0.2$ ) to produce the desired product 50 (1.10 g, 53\%, white solid). ${ }^{1} \mathrm{H}$ NMR (400 MHz, $\left.\mathrm{CDCl}_{3}\right) \delta 7.21(\mathrm{~d}, J=$ $8.0 \mathrm{~Hz}, 1 \mathrm{H}), 7.10-7.07(\mathrm{~m}, 1 \mathrm{H}), 7.01-6.98(\mathrm{~m}, 2 \mathrm{H}), 6.89(\mathrm{~d}, J=8.3 \mathrm{~Hz}, 1 \mathrm{H}), 6.72-6.71(\mathrm{~m}, 1 \mathrm{H}), 3.91$ (s, 3H), $3.84(\mathrm{~s}, 3 \mathrm{H}), 3.62(\mathrm{t}, J=6.8 \mathrm{~Hz}, 2 \mathrm{H}), 3.56(\mathrm{t}, J=6.3 \mathrm{~Hz}, 2 \mathrm{H}), 2.48(\mathrm{q}, J=7.6 \mathrm{~Hz}, 2 \mathrm{H}), 2.34$ $2.27(\mathrm{~m}, 2 \mathrm{H}), 1.08(\mathrm{t}, J=7.6 \mathrm{~Hz}, 3 \mathrm{H}) ;{ }^{13} \mathrm{C} \mathrm{NMR}\left(100 \mathrm{MHz}, \mathrm{CDCl}_{3}\right) \delta 150.0,149.7,145.4,136.6,134.8$, $128.6,127.7,126.5,126.2,122.1,117.1,115.2,112.0,56.2,56.0,52.3,30.8,29.7,28.5,15.4$; IR (nea t) $v_{\max }=2959,2213,1586,1502,1440,1246,1233,1139,1021,803,764\left(\mathrm{~cm}^{-1}\right)$; HRMS (FAB): calcd. for $\mathrm{C}_{20} \mathrm{H}_{24} \mathrm{BrN}_{2} \mathrm{O}_{2} \mathrm{~S}[\mathrm{M}+\mathrm{H}]^{+}$435.0742, found 435.0739 .

$N$-(4-Bromobutyl)- $N$-(3,4-dimethoxyphenyl)-6-ethylbenzo[d]thiazol-2-amine (51). To a solution of compound 35 (1.50 g, $4.77 \mathrm{mmol})$ in dry DMF (24 mL, $0.2 \mathrm{M})$ was added $\mathrm{NaH}($ dry $90 \%, 318 \mathrm{mg}, 11.9$ $\mathrm{mmol}$ ), and the mixture was stirred at $0{ }^{\circ} \mathrm{C}$ for $10 \mathrm{~min}$. To this reaction mixture was added 1,4-dibromobutane $(5.70 \mathrm{~mL}, 47.7 \mathrm{mmol})$, and then the mixture was allowed to warm to room temperature slowly and stirred at $50{ }^{\circ} \mathrm{C}$ for $13 \mathrm{~h}$. Next, the reaction mixture was diluted with $\mathrm{H}_{2} \mathrm{O}$ and extracted with EtOAc. The organic layer was dried over $\mathrm{MgSO}_{4}$, filtered and evaporated in vacuo. The crude product was separated by silica gel column chromatography (hexane/ether, $2: 1, \mathrm{R}_{\mathrm{f}}=0.25$ ) to produce the desired product $51\left(1.70 \mathrm{~g}, 79 \%\right.$, white solid). ${ }^{1} \mathrm{H} \mathrm{NMR}\left(400 \mathrm{MHz}, \mathrm{CDCl}_{3}\right) \delta 7.22(\mathrm{~d}, J=8.0 \mathrm{~Hz}, 1 \mathrm{H}), 7.08$ (dd, $J=8.3,2.1 \mathrm{~Hz}, 1 \mathrm{H}), 7.01-6.98(\mathrm{~m}, 2 \mathrm{H}), 6.90(\mathrm{~d}, J=8.3 \mathrm{~Hz}, 1 \mathrm{H}), 6.72-6.71(\mathrm{~m}, 1 \mathrm{H}), 3.92(\mathrm{~s}, 3 \mathrm{H}), 3.85$ (s, 3H), 3.49-3.43 (m, 4H), 2.48 (q, $J=7.6 \mathrm{~Hz}, 2 \mathrm{H}), 2.06-2.00(\mathrm{~m}, 2 \mathrm{H}), 1.97-1.91(\mathrm{~m}, 2 \mathrm{H}), 1.09$ (t, $J$ $=7.6 \mathrm{~Hz}, 3 \mathrm{H}) ;{ }^{13} \mathrm{C} \mathrm{NMR}\left(100 \mathrm{MHz}, \mathrm{CDCl}_{3}\right) \delta 150.0,149.7,145.3,136.6,134.8,128.4,127.7,126.4$, $126.3,122.0,117.1,115.5,112.0,56.2,56.0,53.1,32.9,29.5,28.5,26.6,15.4$; IR (neat) $v_{\max }=2924$ , 2216, 1585, 1505, 1443, 1254, 1236, 1137, 1023, $811\left(\mathrm{~cm}^{-1}\right)$; HRMS (ESI): calcd. for $\mathrm{C}_{21} \mathrm{H}_{26} \mathrm{BrN}_{2} \mathrm{O}_{2} \mathrm{~S}[\mathrm{M}+\mathrm{H}]^{+}$451.0874, found 451.0866.

$N$-(5-Bromopentyl)- $N$-(3,4-dimethoxyphenyl)-6-ethylbenzo[d] thiazol-2-amine (52). To a solution 
of compound $35(1.50 \mathrm{~g}, 4.77 \mathrm{mmol})$ in dry DMF $(23.9 \mathrm{~mL}, 0.2 \mathrm{M})$ was added $\mathrm{NaH}(60 \%$ in mineral oil, $477 \mathrm{mg}, 11.9 \mathrm{mmol}$ ), and the mixture was stirred at $0{ }^{\circ} \mathrm{C}$ for $10 \mathrm{~min}$. To this reaction mixture was added 1,5-dibromo-pentane $(6.50 \mathrm{~mL}, 47.7 \mathrm{mmol})$, and then the mixture was allowed to warm to room temperature slowly and stirred at $50{ }^{\circ} \mathrm{C}$ for $13 \mathrm{~h}$. Next, the reaction mixture was diluted with $\mathrm{H}_{2} \mathrm{O}$ and extracted with EtOAc. The organic layer was dried over $\mathrm{MgSO}_{4}$, filtered and evaporated in vacuo. The crude product was separated by silica gel column chromatography (hexane/ether, $2: 1, R_{f}=0.25$ ) to produce the desired product $52\left(1.40 \mathrm{~g}, 63 \%\right.$, white solid). ${ }^{1} \mathrm{H}$ NMR $\left(400 \mathrm{MHz}, \mathrm{CDCl}_{3}\right) \delta 7.22(\mathrm{~d}, J=$ $8.0 \mathrm{~Hz}, 1 \mathrm{H}), 7.10-7.08(\mathrm{~m}, 1 \mathrm{H}), 7.01-6.98(\mathrm{~m}, 2 \mathrm{H}), 6.90(\mathrm{~d}, J=8.3 \mathrm{~Hz}, 1 \mathrm{H}), 6.72-6.71(\mathrm{~m}, 1 \mathrm{H}), 3.92$ (s, 3H), $3.86(\mathrm{~s}, 3 \mathrm{H}), 3.46-3.38(\mathrm{~m}, 4 \mathrm{H}), 2.49(\mathrm{q}, J=7.6 \mathrm{~Hz}, 2 \mathrm{H}), 1.95-1.88(\mathrm{~m}, 2 \mathrm{H}), 1.84-1.77$ (m, 2H), 1.63-1.56 (m, 2H), $1.10(\mathrm{t}, J=7.6 \mathrm{~Hz}, 3 \mathrm{H}) ;{ }^{13} \mathrm{C} \mathrm{NMR}\left(100 \mathrm{MHz}, \mathrm{CDCl}_{3}\right) \delta 150.0,149.8,145.3$, 136.6, 135.1, 128.5, 127.7, 126.42, 126.39, 122.2, 117.2, 115.7, 112.1, 56.2, 56.1 53.8, 33.4, 32.4, 28.5, 27.2, 25.2, 15.5; IR (neat) $v_{\max }=2938,2210,1582,1503,1251,1234,1021,839,762\left(\mathrm{~cm}^{-1}\right)$; HRMS (ESI): calcd. for $\mathrm{C}_{22} \mathrm{H}_{28} \mathrm{BrN}_{2} \mathrm{O}_{2} \mathrm{~S}[\mathrm{M}+\mathrm{H}]^{+} 465.1031$, found 465.1028 .

\section{B. Synthetic Procedures for the Preparation of 8-11 and 15-34}

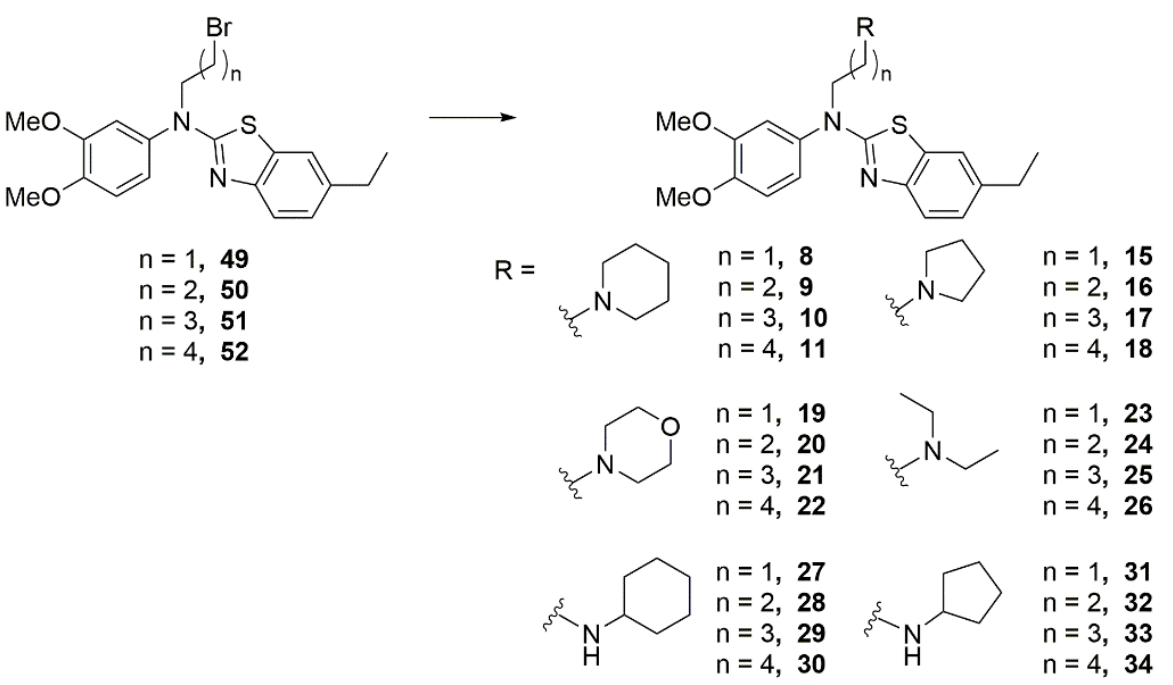

$N$-(3,4-Dimethoxyphenyl)-6-ethyl- $N$-(2-(piperidin-1-yl)ethyl)benzo[d]thiazol-2-amine (8). To a solution of $49(150 \mathrm{mg}, 0.36 \mathrm{mmol})$ in $\mathrm{DMF} / \mathrm{MeCN}(1: 1,3.56 \mathrm{~mL}, 0.1 \mathrm{M})$ was added $\mathrm{K}_{2} \mathrm{CO}_{3}(197$ 
$\mathrm{mg}, 1.42 \mathrm{mmol})$ and piperidine $(352 \mu \mathrm{L}, 3.56 \mathrm{mmol})$. The reaction mixture was stirred at $50{ }^{\circ} \mathrm{C}$ for 13 $\mathrm{h}$ and then diluted with $\mathrm{H}_{2} \mathrm{O}$ and extracted with EtOAc. The organic layer was dried over $\mathrm{MgSO}_{4}$, filtered and evaporated in vacuo. The crude product was separated by silica gel column chromatography $\left(\mathrm{CH}_{2} \mathrm{Cl}_{2} / \mathrm{MeOH}, 20: 1, \mathrm{R}_{\mathrm{f}}=0.2\right)$ to produce the desired product 8 (90.0 mg, 59\%, white solid). ${ }^{1} \mathrm{H}$ NMR $\left(400 \mathrm{MHz}, \mathrm{CDCl}_{3}\right) \delta 7.32(\mathrm{~d}, J=8.0 \mathrm{~Hz}, 1 \mathrm{H}), 7.09-7.07(\mathrm{~m}, 1 \mathrm{H}), 7.00-6.96(\mathrm{~m}, 2 \mathrm{H}), 6.89(\mathrm{~d}, J=8.3$ $\mathrm{Hz}, 1 \mathrm{H}), 6.72-6.71(\mathrm{~m}, 1 \mathrm{H}), 3.91(\mathrm{~s}, 3 \mathrm{H}), 3.85(\mathrm{~s}, 3 \mathrm{H}), 3.55(\mathrm{t}, J=6.8 \mathrm{~Hz}, 2 \mathrm{H}), 2.61(\mathrm{t}, J=6.8 \mathrm{~Hz}, 2 \mathrm{H})$, 2.51-2.41 (m, 6H), 1.60-1.54 (m, 4H), 1.45-1.41 (m, 2H), $1.09(\mathrm{t}, J=7.6 \mathrm{~Hz}, 3 \mathrm{H}) ;{ }^{13} \mathrm{C}$ NMR $(100$ $\left.\mathrm{MHz}, \mathrm{CDCl}_{3}\right) \delta 150.0,149.7,145.1,136.4,135.2,128.4,127.6,127.1,126.3,122.5,117.2,116.0,112.0$, 56.24, 56.21, 56.1, $54.7(2 \mathrm{C}), 51.0,28.5,26.0(2 \mathrm{C}), 24.4,15.5$; IR (neat) $v_{\max }=2934,2214,1584$, 1503, 1398, 1253, 1231, 1135, 1023, $762\left(\mathrm{~cm}^{-1}\right)$; HRMS (ESI): calcd. for $\mathrm{C}_{24} \mathrm{H}_{32} \mathrm{~N}_{3} \mathrm{O}_{2} \mathrm{~S}[\mathrm{M}+\mathrm{H}]^{+}$ 426.2210, found 426.2204 .

$N$-(3,4-Dimethoxyphenyl)-6-ethyl- $N$-(3-(piperidin-1-yl)propyl)benzo[ $d]$ thiazol-2-amine (9). To a solution of $\mathbf{5 0}$ (150 mg, $0.34 \mathrm{mmol})$ in DMF/MeCN (1:1, $3.4 \mathrm{~mL}, 0.1 \mathrm{M})$ was added $\mathrm{K}_{2} \mathrm{CO}_{3}(188 \mathrm{mg}$, $1.36 \mathrm{mmol})$ and piperidine $(336 \mu \mathrm{L}, 3.40 \mathrm{mmol})$. The reaction mixture was stirred at $50{ }^{\circ} \mathrm{C}$ for $15 \mathrm{~h}$ and then diluted with $\mathrm{H}_{2} \mathrm{O}$ and extracted with EtOAc. The organic layer was dried over $\mathrm{MgSO}_{4}$, filtered and evaporated in vacuo. The crude product was separated by silica gel column chromatography $\left(\mathrm{CH}_{2} \mathrm{Cl}_{2} / \mathrm{MeOH}, 20: 1, \mathrm{R}_{\mathrm{f}}=0.2\right)$ to produce the desired product 9 (102 $\mathrm{mg}, 68 \%$, ivory solid). ${ }^{1} \mathrm{H}$ NMR $\left(400 \mathrm{MHz}, \mathrm{CDCl}_{3}\right) \delta 7.23(\mathrm{~d}, J=8.0 \mathrm{~Hz}, 1 \mathrm{H}), 7.09(\mathrm{dd}, J=8.3,2.1 \mathrm{~Hz}, 1 \mathrm{H}), 7.00-6.97(\mathrm{~m}, 2 \mathrm{H}), 6.90$ (d, $J=8.4 \mathrm{~Hz}, 1 \mathrm{H}), 6.71-6.70(\mathrm{~m}, 1 \mathrm{H}), 3.92(\mathrm{~s}, 3 \mathrm{H}), 3.85$ (s, 3H), 3.49 (t, $J=7.3 \mathrm{~Hz}, 2 \mathrm{H}), 2.50-2.39$ (m, 8H), 2.00-1.92 (m, 2H), 1.60-1.54 (m, 4H), 1.45-1.42 (m, 2H), 1.09 (t, J=7.6 Hz, 3H); ${ }^{13} \mathrm{C}$ NMR $\left(100 \mathrm{MHz}, \mathrm{CDCl}_{3}\right) \delta 150.0,149.8,145.2,136.6,135.2,128.5,127.7,126.4$ (2C), 122.4, 117.2, 115.8, 112.0, 56.2, 56.1, 55.8, 54.6(2C), 52.4, 28.6, $26.0(2 \mathrm{C}), 25.3,24.5,15.5$; IR (neat) $v_{\max }=2933,221$ $3,1584,1503,1400,1252,1230,1136,1023,851,762\left(\mathrm{~cm}^{-1}\right)$; HRMS (ESI): calcd. for $\mathrm{C}_{25} \mathrm{H}_{34} \mathrm{~N}_{3} \mathrm{O}_{2} \mathrm{~S}[\mathrm{M}+\mathrm{H}]^{+} 440.2366$, found 440.2370 .

$N$-(3,4-Dimethoxyphenyl)-6-ethyl- $N$-(4-(piperidin-1-yl)butyl)benzo[d]thiazol-2-amine (10). To a solution of $51(130 \mathrm{mg}, 0.29 \mathrm{mmol})$ in DMF/MeCN (1:1, $3.0 \mathrm{~mL}, 0.1 \mathrm{M})$ was added $\mathrm{K}_{2} \mathrm{CO}_{3}(160 \mathrm{mg}$, 
$1.16 \mathrm{mmol})$ and piperidine $(286 \mu \mathrm{L}, 2.89 \mathrm{mmol})$. The reaction mixture was stirred at $50{ }^{\circ} \mathrm{C}$ for $15 \mathrm{~h}$ and then diluted with $\mathrm{H}_{2} \mathrm{O}$ and extracted with EtOAc. The organic layer was dried over $\mathrm{MgSO}_{4}$, filtered and evaporated in vacuo. The crude product was separated by silica gel column chromatography $\left(\mathrm{CH}_{2} \mathrm{Cl}_{2} / \mathrm{MeOH}, 20: 1, \mathrm{R}_{\mathrm{f}}=0.2\right)$ to produce the desired product 10 (87.0 $\mathrm{mg}, 66 \%$, yellow oil). ${ }^{1} \mathrm{H} \mathrm{NMR}$ $\left(400 \mathrm{MHz}, \mathrm{CDCl}_{3}\right) \delta 7.20(\mathrm{~d}, J=8.0 \mathrm{~Hz}, 1 \mathrm{H}), 7.06(\mathrm{dd}, J=8.2,2.1 \mathrm{~Hz}, 1 \mathrm{H}), 6.98-6.95(\mathrm{~m}, 2 \mathrm{H}), 6.88$ $(\mathrm{d}, J=8.4 \mathrm{~Hz}, 1 \mathrm{H}), 6.69-6.68(\mathrm{~m}, 1 \mathrm{H}), 3.90(\mathrm{~s}, 3 \mathrm{H}), 3.83(\mathrm{~s}, 3 \mathrm{H}), 3.43(\mathrm{t}, J=7.1 \mathrm{~Hz}, 2 \mathrm{H}), 2.49-2.39$ $(\mathrm{m}, 8 \mathrm{H}), 1.81-1.60(\mathrm{~m}, 8 \mathrm{H}), 1.46-1.43(\mathrm{~m}, 2 \mathrm{H}), 1.07(\mathrm{t}, J=7.6 \mathrm{~Hz}, 3 \mathrm{H}) ;{ }^{13} \mathrm{C}$ NMR $\left(100 \mathrm{MHz}, \mathrm{CDCl}_{3}\right)$ $\delta 150.0,149.7,145.2,136.5,135.1,128.4,127.7,126.4,126.3,122.2,117.2,115.7,112.0,58.6,56.2$, $56.0,54.4(2 \mathrm{C}), 53.8,28.5,26.0,25.5(2 \mathrm{C}), 24.1,23.4,15.4$; IR (neat) $v_{\max }=2935,2213,1584,15$ 03, 1441, 1253, 1230, 1136, 1023, $728\left(\mathrm{~cm}^{-1}\right)$; HRMS (ESI): calcd. for $\mathrm{C}_{26} \mathrm{H}_{36} \mathrm{~N}_{3} \mathrm{O}_{2} \mathrm{~S}[\mathrm{M}+\mathrm{H}]^{+}$ 454.2523, found 454.2527 .

$N$-(3,4-Dimethoxyphenyl)-6-ethyl- $N$-(5-(piperidin-1-yl)pentyl)benzo[d]thiazol-2-amine (11). To a solution of 52 (130 mg, $0.28 \mathrm{mmol})$ in DMF/MeCN $(1: 1,2.8 \mathrm{~mL}, 0.1 \mathrm{M})$ was added $\mathrm{K}_{2} \mathrm{CO}_{3}(154 \mathrm{mg}$, $1.12 \mathrm{mmol})$ and piperidine $(278 \mu \mathrm{L}, 2.81 \mathrm{mmol})$. The reaction mixture was stirred at $50^{\circ} \mathrm{C}$ for $14 \mathrm{~h}$ and then diluted with $\mathrm{H}_{2} \mathrm{O}$ and extracted with EtOAc. The organic layer was dried over $\mathrm{MgSO}_{4}$, filtered and evaporated in vacuo. The crude product was separated by silica gel column chromatography $\left(\mathrm{CH}_{2} \mathrm{Cl}_{2} / \mathrm{MeOH}, 30: 1, \mathrm{R}_{\mathrm{f}}=0.2\right)$ to produce the desired product 11 (109 mg, 83\%, ivory solid). ${ }^{1} \mathrm{H}$ NMR $\left(400 \mathrm{MHz}, \mathrm{CDCl}_{3}\right) \delta 7.20(\mathrm{~d}, J=8.0 \mathrm{~Hz}, 1 \mathrm{H}), 7.08(\mathrm{dd}, J=8.2,2.1 \mathrm{~Hz}, 1 \mathrm{H}), 6.99-6.96(\mathrm{~m}, 2 \mathrm{H}), 6.89$ $(\mathrm{d}, J=8.3 \mathrm{~Hz}, 1 \mathrm{H}), 6.70(\mathrm{~d}, J=2.0 \mathrm{~Hz}, 1 \mathrm{H}), 3.91(\mathrm{~s}, 3 \mathrm{H}), 3.85(\mathrm{~s}, 3 \mathrm{H}), 3.41(\mathrm{t}, J=7.4 \mathrm{~Hz}, 2 \mathrm{H}), 2.50$ $2.36(\mathrm{~m}, 8 \mathrm{H}), 1.82-1.75(\mathrm{~m}, 2 \mathrm{H}), 1.67-1.56(\mathrm{~m}, 6 \mathrm{H}), 1.48-1.40(\mathrm{~m}, 4 \mathrm{H}), 1.08(\mathrm{t}, J=7.6 \mathrm{~Hz}, 3 \mathrm{H}) ;{ }^{13} \mathrm{C}$ $\operatorname{NMR}\left(100 \mathrm{MHz}, \mathrm{CDCl}_{3}\right) \delta 150.0,149.8,145.2,136.6,135.1,128.4,127.7,126.41,126.36,122.3,117.3$, $115.8,112.0,59.0,56.2,56.1,54.5(2 \mathrm{C}), 53.9,28.5,27.8,26.1,25.5$ (2C), 24.6, 24.2, 15.5; IR (neat) $v_{\max }=2934,2213,1584,1503,1321,1253,1231,1136,1022,762\left(\mathrm{~cm}^{-1}\right)$; HRMS (FAB): calcd. for $\mathrm{C}_{27} \mathrm{H}_{38} \mathrm{~N}_{3} \mathrm{O}_{2} \mathrm{~S}[\mathrm{M}+\mathrm{H}]^{+}$468.2685, found 468.2690.

$N$-(3,4-Dimethoxyphenyl)-6-ethyl- $N$-(2-(pyrrolidin-1-yl)ethyl)benzo[d] thiazol-2-amine (15). To a solution of 49 (100 mg, $0.24 \mathrm{mmol})$ in DMF/MeCN $(1: 1,2.4 \mathrm{~mL}, 0.1 \mathrm{M})$ was added $\mathrm{K}_{2} \mathrm{CO}_{3}(131 \mathrm{mg}$, 
$0.95 \mathrm{mmol})$ and pyrrolidine $(200 \mu \mathrm{L}, 2.37 \mathrm{mmol})$. The reaction mixture was stirred at $50{ }^{\circ} \mathrm{C}$ for $13 \mathrm{~h}$ and then diluted with $\mathrm{H}_{2} \mathrm{O}$ and extracted with EtOAc. The organic layer was dried over $\mathrm{MgSO}_{4}$, filtered and evaporated in vacuo. The crude product was separated by silica gel column chromatography $\left(\mathrm{CH}_{2} \mathrm{Cl}_{2} / \mathrm{MeOH}, 15: 1, \mathrm{R}_{\mathrm{f}}=0.2\right)$ to produce the desired product 15 (87.0 $\mathrm{mg}, 89 \%$, yellow oil). ${ }^{1} \mathrm{H}$ NMR $\left(400 \mathrm{MHz}, \mathrm{CDCl}_{3}\right) \delta 7.29(\mathrm{~d}, J=8.0 \mathrm{~Hz}, 1 \mathrm{H}), 7.08(\mathrm{dd}, J=8.3,2.0 \mathrm{~Hz}, 1 \mathrm{H}), 7.00-6.96(\mathrm{~m}, 2 \mathrm{H}), 6.89$ $(\mathrm{d}, J=8.3 \mathrm{~Hz}, 1 \mathrm{H}), 6.71-6.70(\mathrm{~m}, 1 \mathrm{H}), 3.91(\mathrm{~s}, 3 \mathrm{H}), 3.85(\mathrm{~s}, 3 \mathrm{H}), 3.57(\mathrm{t}, J=7.2 \mathrm{~Hz}, 2 \mathrm{H}), 2.82(\mathrm{t}, J=$ $7.2 \mathrm{~Hz}, 2 \mathrm{H}), 2.57-2.56(\mathrm{~m}, 4 \mathrm{H}), 2.48$ (q, $J=7.6 \mathrm{~Hz}, 2 \mathrm{H}), 1.80-1.77(\mathrm{~m}, 4 \mathrm{H}), 1.09$ (t, $J=7.6 \mathrm{~Hz}, 3 \mathrm{H})$; ${ }^{13} \mathrm{C} \mathrm{NMR}\left(100 \mathrm{MHz}, \mathrm{CDCl}_{3}\right) \delta 150.0,149.7,145.2,136.6,135.0,128.4,127.7,126.9,126.4,122.4$, $117.3,115.9,112.0,56.2,56.1,54.2(2 \mathrm{C}), 53.4,52.5,28.5,23.7$ (2C) 15.5; IR (neat) $v_{\max }=2963,2214$, 1584, 1503, 1440, 1252, 1230, 1135, 1022, $806\left(\mathrm{~cm}^{-1}\right)$; HRMS (ESI): calcd. for $\mathrm{C}_{23} \mathrm{H}_{30} \mathrm{~N}_{3} \mathrm{O}_{2} \mathrm{~S}[\mathrm{M}+\mathrm{H}]^{+}$ 412.2053, found 412.2048.

$N$-(3,4-Dimethoxyphenyl)-6-ethyl- $N$-(3-(pyrrolidin-1-yl)propyl)benzo[d] thiazol-2-amine (16). To a solution of $\mathbf{5 0}(150 \mathrm{mg}, 0.34 \mathrm{mmol})$ in $\mathrm{DMF} / \mathrm{MeCN}(1: 1,3.4 \mathrm{~mL}, 0.1 \mathrm{M})$ was added $\mathrm{K}_{2} \mathrm{CO}_{3}(191 \mathrm{mg}$, $1.38 \mathrm{mmol})$ and pyrrolidine $(280 \mu \mathrm{L}, 3.41 \mathrm{mmol})$. The reaction mixture was stirred at $50{ }^{\circ} \mathrm{C}$ for $15 \mathrm{~h}$ and then diluted with $\mathrm{H}_{2} \mathrm{O}$ and extracted with EtOAc. The organic layer was dried over $\mathrm{MgSO}_{4}$, filtered and evaporated in vacuo. The crude product was separated by silica gel column chromatography $\left(\mathrm{CH}_{2} \mathrm{Cl}_{2} / \mathrm{MeOH}, 20: 1, \mathrm{R}_{\mathrm{f}}=0.2\right)$ to produce the desired product 16 (74.0 $\mathrm{mg}, 50 \%$, yellow oil). ${ }^{1} \mathrm{H}$ NMR $\left(400 \mathrm{MHz}, \mathrm{CDCl}_{3}\right) \delta 7.22(\mathrm{~d}, J=8.0 \mathrm{~Hz}, 1 \mathrm{H}), 7.07(\mathrm{dd}, J=8.3,2.1 \mathrm{~Hz}, 1 \mathrm{H}), 6.99-6.96(\mathrm{~m}, 2 \mathrm{H}), 6.88$ (d, $J=8.3 \mathrm{~Hz}, 1 \mathrm{H}), 6.70-6.69(\mathrm{~m}, 1 \mathrm{H}), 3.90(\mathrm{~s}, 3 \mathrm{H}), 3.84(\mathrm{~s}, 3 \mathrm{H}), 3.51(\mathrm{t}, J=7.2 \mathrm{~Hz}, 2 \mathrm{H}), 2.68(\mathrm{t}, J=$ $7.3 \mathrm{~Hz}, 2 \mathrm{H}), 2.62-2.58(\mathrm{~m}, 4 \mathrm{H}), 2.46(\mathrm{q}, J=7.6 \mathrm{~Hz}, 2 \mathrm{H}), 2.06-1.99(\mathrm{~m}, 2 \mathrm{H}), 1.82-1.78(\mathrm{~m}, 4 \mathrm{H}), 1.07$ $(\mathrm{t}, J=7.6 \mathrm{~Hz}, 3 \mathrm{H}) ;{ }^{13} \mathrm{C}$ NMR $\left(100 \mathrm{MHz}, \mathrm{CDCl}_{3}\right) \delta 150.0,149.7,145.2,136.5,135.0,128.5,127.7$, $126.42,126.35,122.2,117.2,115.6,112.0,56.2,56.1,54.1(2 \mathrm{C}), 53.1,52.3,28.5,27.0,23.5(2 \mathrm{C}), 15.4$; IR (neat) $v_{\max }=2985,2214,1585,1503,1442,1253,1231,1137,1023,728\left(\mathrm{~cm}^{-1}\right)$; HRMS (FAB): calcd. for $\mathrm{C}_{24} \mathrm{H}_{32} \mathrm{~N}_{3} \mathrm{O}_{2} \mathrm{~S}[\mathrm{M}+\mathrm{H}]^{+} 426.2215$, found 426.2220 .

$N$-(3,4-Dimethoxyphenyl)-6-ethyl- $N$-(4-(pyrrolidin-1-yl)butyl)benzo[d] thiazol-2-amine (17). To a solution of 51 (138 mg, $0.31 \mathrm{mmol})$ in DMF/MeCN $(1: 1,3.0 \mathrm{~mL}, 0.1 \mathrm{M})$ was added $\mathrm{K}_{2} \mathrm{CO}_{3}(170 \mathrm{mg}$, 
$1.23 \mathrm{mmol})$ and pyrrolidine $(256 \mu \mathrm{L}, 3.07 \mathrm{mmol})$. The reaction mixture was stirred at $50{ }^{\circ} \mathrm{C}$ for $15 \mathrm{~h}$ and then diluted with $\mathrm{H}_{2} \mathrm{O}$ and extracted with EtOAc. The organic layer was dried over $\mathrm{MgSO}_{4}$, filtered and evaporated in vacuo. The crude product was separated by silica gel column chromatography $\left(\mathrm{CH}_{2} \mathrm{Cl}_{2} / \mathrm{MeOH}, 20: 1, \mathrm{R}_{\mathrm{f}}=0.2\right)$ to produce the desired product 17 (101 $\mathrm{mg}, 74 \%$, yellow oil). ${ }^{1} \mathrm{H}$ NMR $\left(400 \mathrm{MHz}, \mathrm{CDCl}_{3}\right) \delta 7.20(\mathrm{~d}, J=8.0 \mathrm{~Hz}, 1 \mathrm{H}), 7.07(\mathrm{dd}, J=8.3,2.1 \mathrm{~Hz}, 1 \mathrm{H}), 6.98-6.96(\mathrm{~m}, 2 \mathrm{H}), 6.88$ (d, $J=8.4 \mathrm{~Hz}, 1 \mathrm{H}), 6.69-6.68(\mathrm{~m}, 1 \mathrm{H}), 3.90(\mathrm{~s}, 3 \mathrm{H}), 3.84(\mathrm{~s}, 3 \mathrm{H}), 3.44$ (t, $J=6.9 \mathrm{~Hz}, 2 \mathrm{H}), 2.69-2.61$ (m, 6H), $2.46(\mathrm{q}, J=7.6 \mathrm{~Hz}, 2 \mathrm{H}), 1.87-1.74(\mathrm{~m}, 8 \mathrm{H}), 1.07(\mathrm{t}, J=7.6 \mathrm{~Hz}, 3 \mathrm{H}) ;{ }^{13} \mathrm{C}$ NMR $(100 \mathrm{MHz}$, $\left.\mathrm{CDCl}_{3}\right) \delta 150.0,149.7,145.3,136.5,135.0,128.4,127.7,126.4,126.3,122.1,117.2,115.6,112.0,56.2$, $56.1,55.7,54.1(2 \mathrm{C}), 53.7,28.5,25.9,25.1,23.5$ (2C), 15.4; IR (neat) $v_{\max }=2963,2213,1584,1503$, 1441, 1253, 1230, 1136, 1022, $728\left(\mathrm{~cm}^{-1}\right)$; HRMS (ESI): calcd. for $\mathrm{C}_{25} \mathrm{H}_{34} \mathrm{~N}_{3} \mathrm{O}_{2} \mathrm{~S}[\mathrm{M}+\mathrm{H}]^{+} 440.2366$, found 440.2364 .

$N$-(3,4-Dimethoxyphenyl)-6-ethyl- $N$-(5-(pyrrolidin-1-yl)pentyl)benzo[d] thiazol-2-amine (18). To a solution of 52 (150 mg, $0.32 \mathrm{mmol})$ in DMF/MeCN $(1: 1,3.2 \mathrm{~mL}, 0.1 \mathrm{M})$ was added $\mathrm{K}_{2} \mathrm{CO}_{3}(177 \mathrm{mg}$, $1.28 \mathrm{mmol})$ and pyrrolidine $(270 \mu \mathrm{L}, 3.21 \mathrm{mmol})$. The reaction mixture was stirred at $50{ }^{\circ} \mathrm{C}$ for $18 \mathrm{~h}$ and then diluted with $\mathrm{H}_{2} \mathrm{O}$ and extracted with EtOAc. The organic layer was dried over $\mathrm{MgSO}_{4}$, filtered and evaporated in vacuo. The crude product was separated by silica gel column chromatography $\left(\mathrm{CH}_{2} \mathrm{Cl}_{2} / \mathrm{MeOH}, 20: 1, \mathrm{R}_{\mathrm{f}}=0.2\right)$ to produce the desired product $18\left(87.0 \mathrm{mg}, 60 \%\right.$, yellow oil). ${ }^{1} \mathrm{H}$ NMR $\left(400 \mathrm{MHz}, \mathrm{CDCl}_{3}\right) \delta 7.20(\mathrm{~d}, J=8.0 \mathrm{~Hz}, 1 \mathrm{H}), 7.08(\mathrm{dd}, J=8.3,2.0 \mathrm{~Hz}, 1 \mathrm{H}), 6.99-6.97(\mathrm{~m}, 2 \mathrm{H}), 6.90$ (d, $J=8.3 \mathrm{~Hz}, 1 \mathrm{H}), 6.70-6.69(\mathrm{~m}, 1 \mathrm{H}), 3.91(\mathrm{~s}, 3 \mathrm{H}), 3.85(\mathrm{~s}, 3 \mathrm{H}), 3.43(\mathrm{t}, J=7.3 \mathrm{~Hz}, 2 \mathrm{H}), 2.90-2.77$ (m, 4H), $2.70(\mathrm{t}, J=7.9 \mathrm{~Hz}, 2 \mathrm{H}), 2.47(\mathrm{q}, J=7.6 \mathrm{~Hz}, 2 \mathrm{H}), 1.94-1.89(\mathrm{~m}, 4 \mathrm{H}), 1.84-1.70(\mathrm{~m}, 4 \mathrm{H}), 1.55-$ $1.47(\mathrm{~m}, 2 \mathrm{H}), 1.09(\mathrm{t}, J=7.6 \mathrm{~Hz}, 3 \mathrm{H}) ;{ }^{13} \mathrm{C} \mathrm{NMR}\left(100 \mathrm{MHz}, \mathrm{CDCl}_{3}\right) \delta 150.0,149.8,145.3,136.6,135.0$, $128.4,127.8,126.44,126.38,122.2,117.3,115.7,112.1,56.3,56.1,55.9,54.0(2 \mathrm{C}), 53.7,28.5,27.6$, $27.2,24.3,23.5$ (2C), 15.5; IR (neat) $v_{\max }=2934,2213,1736,1584,1503,1441,1251,1231,1136$, 1023, $806\left(\mathrm{~cm}^{-1}\right)$; HRMS (FAB): calcd. for $\mathrm{C}_{26} \mathrm{H}_{36} \mathrm{~N}_{3} \mathrm{O}_{2} \mathrm{~S}[\mathrm{M}+\mathrm{H}]^{+} 454.2528$, found 454.2520.

$N$-(3,4-Dimethoxyphenyl)-6-ethyl- $N$-(2-morpholinoethyl)benzo[ $d]$ thiazol-2-amine $\quad(19) . \quad$ To a solution of $49(20 \mathrm{mg}, 0.05 \mathrm{mmol})$ in $\mathrm{DMF} / \mathrm{MeCN}(1: 1,0.5 \mathrm{~mL}, 0.1 \mathrm{M})$ was added $\mathrm{K}_{2} \mathrm{CO}_{3}(26 \mathrm{mg}, 0.19$ 
mmol) and morpholine ( $43 \mu \mathrm{L}, 0.5 \mathrm{mmol})$. The reaction mixture was stirred at $50{ }^{\circ} \mathrm{C}$ for $12 \mathrm{~h}$ and then diluted with $\mathrm{H}_{2} \mathrm{O}$ and extracted with EtOAc. The organic layer was dried over $\mathrm{MgSO}_{4}$, filtered and evaporated in vacuo. The crude product was separated by silica gel column chromatography $\left(\mathrm{CH}_{2} \mathrm{Cl}_{2} / \mathrm{MeOH}, 30: 1, \mathrm{R}_{\mathrm{f}}=0.2\right)$ to produce the desired product 19 (18.0 $\mathrm{mg}, 84 \%$, ivory solid). ${ }^{1} \mathrm{H} \mathrm{NMR}$ $\left(400 \mathrm{MHz}, \mathrm{CDCl}_{3}\right) \delta 7.33(\mathrm{~d}, J=8.0 \mathrm{~Hz}, 1 \mathrm{H}), 7.09-7.07(\mathrm{~m}, 1 \mathrm{H}), 7.00-6.97(\mathrm{~m}, 2 \mathrm{H}), 6.90(\mathrm{~d}, J=8.3$ Hz, 1H), 6.70-6.99 (m, 1H), $3.92(\mathrm{~s}, 3 \mathrm{H}), 3.85(\mathrm{~s}, 3 \mathrm{H}), 3.73-3.71(\mathrm{~m}, 4 \mathrm{H}), 3.58(\mathrm{t}, J=6.3 \mathrm{~Hz}, 2 \mathrm{H}), 2.67$ (t, $J=6.1 \mathrm{~Hz}, 2 \mathrm{H}), 2.51-2.45(\mathrm{~m}, 6 \mathrm{H}), 1.09(\mathrm{t}, J=7.6 \mathrm{~Hz}, 3 \mathrm{H}) ;{ }^{13} \mathrm{C} \mathrm{NMR}\left(100 \mathrm{MHz}, \mathrm{CDCl}_{3}\right) \delta 150.0$, $149.8,145.3,136.5,135.0,128.4,127.7,127.0,126.3,122.2,117.2,115.9,112.0,66.9(2 \mathrm{C}), 56.2,56.1$, $55.9,53.6$ (2C), 50.2, 28.5, 15.5; IR (neat) $v_{\max }=2934,2214,1584,1503,1440,1252,1230,1115$, 1022, $729\left(\mathrm{~cm}^{-1}\right)$; HRMS (ESI): calcd. for $\mathrm{C}_{23} \mathrm{H}_{30} \mathrm{~N}_{3} \mathrm{O}_{3} \mathrm{~S}[\mathrm{M}+\mathrm{H}]^{+} 428.2002$, found 428.1996 .

$N$-(3,4-Dimethoxyphenyl)-6-ethyl- $N$-(3-morpholinopropyl)benzo[d]thiazol-2-amine (20). То a solution of $\mathbf{5 0}$ (100 mg, $0.23 \mathrm{mmol})$ in DMF/MeCN (1:1, $2.4 \mathrm{~mL}, 0.1 \mathrm{M})$ was added $\mathrm{K}_{2} \mathrm{CO}_{3}(127 \mathrm{mg}$, $0.92 \mathrm{mmol})$ and morpholine $(200 \mu \mathrm{L}, 2.30 \mathrm{mmol})$. The reaction mixture was stirred at $50{ }^{\circ} \mathrm{C}$ for $12 \mathrm{~h}$ and then diluted with $\mathrm{H}_{2} \mathrm{O}$ and extracted with EtOAc. The organic layer was dried over $\mathrm{MgSO}_{4}$, filtered and evaporated in vacuo. The crude product was separated by silica gel column chromatography $\left(\mathrm{CH}_{2} \mathrm{Cl}_{2} / \mathrm{MeOH}, 30: 1, \mathrm{R}_{\mathrm{f}}=0.25\right)$ to produce the desired product $\mathbf{2 0}\left(100 \mathrm{mg}, 98 \%\right.$, yellow oil). ${ }^{1} \mathrm{H}$ NMR $\left(400 \mathrm{MHz}, \mathrm{CDCl}_{3}\right) \delta 7.22(\mathrm{~d}, J=8.0 \mathrm{~Hz}, 1 \mathrm{H}), 7.09-7.06(\mathrm{~m}, 1 \mathrm{H}), 6.99-6.96(\mathrm{~m}, 2 \mathrm{H}), 6.89(\mathrm{~d}, J=8.3$ $\mathrm{Hz}, 1 \mathrm{H}), 6.71-6.70(\mathrm{~m}, 1 \mathrm{H}), 3.90(\mathrm{~s}, 3 \mathrm{H}), 3.84(\mathrm{~s}, 3 \mathrm{H}), 3.68-3.66(\mathrm{~m}, 4 \mathrm{H}), 3.51(\mathrm{t}, J=7.2 \mathrm{~Hz}, 2 \mathrm{H})$, $2.50-2.41(\mathrm{~m}, 8 \mathrm{H}), 1.97-1.90(\mathrm{~m}, 2 \mathrm{H}), 1.08(\mathrm{t}, J=7.6 \mathrm{~Hz}, 3 \mathrm{H}) ;{ }^{13} \mathrm{C} \mathrm{NMR}\left(100 \mathrm{MHz}, \mathrm{CDCl}_{3}\right) \delta 150.1$, $149.8,145.3,136.6,135.1,128.4,127.8,126.4,126.3,122.2,117.2,115.7,112.0,66.9(2 \mathrm{C}), 56.2,56.1$, $55.4,53.6,52.1,29.8,28.6,24.8,15.5$; IR (neat) $v_{\max }=2935,2213,1584,1503,1441,1252,1230$, 1115, 1022, $728\left(\mathrm{~cm}^{-1}\right)$; HRMS (ESI): calcd. for $\mathrm{C}_{24} \mathrm{H}_{32} \mathrm{~N}_{3} \mathrm{O}_{3} \mathrm{~S}[\mathrm{M}+\mathrm{H}]^{+} 442.2159$, found 442.2162.

$N$-(3,4-Dimethoxyphenyl)-6-ethyl- $N$-(4-morpholinobutyl)benzo[d] thiazol-2-amine (21). To a solution of $51(134 \mathrm{mg}, 0.30 \mathrm{mmol})$ in $\mathrm{DMF} / \mathrm{MeCN}(1: 1,3.0 \mathrm{~mL}, 0.1 \mathrm{M})$ was added $\mathrm{K}_{2} \mathrm{CO}_{3}(165 \mathrm{mg}$, $1.19 \mathrm{mmol})$ and morpholine $(260 \mu \mathrm{L}, 2.98 \mathrm{mmol})$. The reaction mixture was stirred at $50{ }^{\circ} \mathrm{C}$ for $15 \mathrm{~h}$ and then diluted with $\mathrm{H}_{2} \mathrm{O}$ and extracted with EtOAc. The organic layer was dried over $\mathrm{MgSO}_{4}$, filtered 
and evaporated in vacuo. The crude product was separated by silica gel column chromatography $\left(\mathrm{CH}_{2} \mathrm{Cl}_{2} / \mathrm{MeOH}, 30: 1, \mathrm{R}_{\mathrm{f}}=0.2\right)$ to produce the desired product $21\left(125 \mathrm{mg}, 92 \%\right.$, white solid). ${ }^{1} \mathrm{H}$ NMR $\left(400 \mathrm{MHz}, \mathrm{CDCl}_{3}\right) \delta 7.21(\mathrm{~d}, J=8.0 \mathrm{~Hz}, 1 \mathrm{H}), 7.09-7.06(\mathrm{~m}, 1 \mathrm{H}), 6.99-6.97(\mathrm{~m}, 2 \mathrm{H}), 6.89(\mathrm{~d}, J=8.4$ $\mathrm{Hz}, 1 \mathrm{H}), 6.70-6.69(\mathrm{~m}, 1 \mathrm{H}), 3.91(\mathrm{~s}, 3 \mathrm{H}), 3.84(\mathrm{~s}, 3 \mathrm{H}), 3.71-3.68(\mathrm{~m}, 4 \mathrm{H}), 3.45(\mathrm{t}, J=7.3 \mathrm{~Hz}, 2 \mathrm{H})$, 2.50-2.36 (m, 8H), 1.85-1.77 (m, 2H), $1.68-1.61(\mathrm{~m}, 2 \mathrm{H}), 1.08(\mathrm{t}, J=7.6 \mathrm{~Hz}, 3 \mathrm{H}) ;{ }^{13} \mathrm{C}$ NMR $(100$ $\left.\mathrm{MHz}, \mathrm{CDCl}_{3}\right) \delta 150.0,149.8,145.2,136.6,135.2,128.4,127.7,126.4,126.3,122.2,117.2,115.7,112.0$ 67.0 (2C), 58.4, 56.2, 56.1, 53.9, 53.7 (2C), 28.5, 25.9, 23.4, 15.5; IR (neat) $v_{\max }=2934,2213,1677$, 1584, 1503, 1253, 1231, 1116, 1022, $733\left(\mathrm{~cm}^{-1}\right)$; HRMS (FAB): calcd. for $\mathrm{C}_{25} \mathrm{H}_{34} \mathrm{~N}_{3} \mathrm{O}_{3} \mathrm{~S}[\mathrm{M}+\mathrm{H}]^{+}$ 456.2321, found 456.2313.

$N$-(3,4-Dimethoxyphenyl)-6-ethyl- $N$-(5-morpholinopentyl)benzo[d] thiazol-2-amine (22). To a solution of $52(150 \mathrm{mg}, 0.32 \mathrm{mmol})$ in $\mathrm{DMF} / \mathrm{MeCN}(1: 1,3.2 \mathrm{~mL}, 0.1 \mathrm{M})$ was added $\mathrm{K}_{2} \mathrm{CO}_{3}(179 \mathrm{mg}$, $1.30 \mathrm{mmol})$ and morpholine $(283 \mu \mathrm{L}, 3.28 \mathrm{mmol})$. The reaction mixture was stirred at $50{ }^{\circ} \mathrm{C}$ for $13 \mathrm{~h}$ and then diluted with $\mathrm{H}_{2} \mathrm{O}$ and extracted with EtOAc. The organic layer was dried over $\mathrm{MgSO}_{4}$, filtered and evaporated in vacuo. The crude product was separated by silica gel column chromatography $\left(\mathrm{CH}_{2} \mathrm{Cl}_{2} / \mathrm{MeOH}, 30: 1, \mathrm{R}_{\mathrm{f}}=0.2\right)$ to produce the desired product $22(110 \mathrm{mg}, 72 \%$, yellow oil $) .{ }^{1} \mathrm{H}$ NMR $\left(400 \mathrm{MHz}, \mathrm{CDCl}_{3}\right) \delta 7.21(\mathrm{~d}, J=8.0 \mathrm{~Hz}, 1 \mathrm{H}), 7.08(\mathrm{dd}, J=8.3,2.1 \mathrm{~Hz}, 1 \mathrm{H}), 6.99-6.96(\mathrm{~m}, 2 \mathrm{H}), 6.89$ (d, $J=8.4 \mathrm{~Hz}, 1 \mathrm{H}), 6.70-6.69(\mathrm{~m}, 1 \mathrm{H}), 3.91(\mathrm{~s}, 3 \mathrm{H}), 3.84(\mathrm{~s}, 3 \mathrm{H}), 3.70-3.68(\mathrm{~m}, 4 \mathrm{H}), 3.41(\mathrm{t}, J=7.4$ $\mathrm{Hz}, 2 \mathrm{H}), 2.50-2.41(\mathrm{~m}, 6 \mathrm{H}), 2.33(\mathrm{t}, J=7.4 \mathrm{~Hz}, 2 \mathrm{H}), 1.83-1.75(\mathrm{~m}, 2 \mathrm{H}), 1.56-1.43(\mathrm{~m}, 4 \mathrm{H}), 1.08(\mathrm{t}, J$ $=7.6 \mathrm{~Hz}, 3 \mathrm{H}) ;{ }^{13} \mathrm{C} \mathrm{NMR}\left(100 \mathrm{MHz}, \mathrm{CDCl}_{3}\right) \delta 150.0,149.7,145.2,136.6,135.2,128.4,127.7,126.4$, $126.3,122.2,117.3,115.7,112.0,67.0(2 \mathrm{C}), 58.8,56.2,56.1,53.9,53.8(2 \mathrm{C}), 28.5,27.9,26.2,24.4$, 15.5; IR (neat) $v_{\max }=2936,2213,1584,1503,1398,1253,1231,1116,1022,731\left(\mathrm{~cm}^{-1}\right) ;$ HRMS (ESI): calcd. for $\mathrm{C}_{26} \mathrm{H}_{36} \mathrm{~N}_{3} \mathrm{O}_{3} \mathrm{~S}[\mathrm{M}+\mathrm{H}]^{+} 470.2472$, found 470.2464 .

$N^{1}$-(3,4-Dimethoxyphenyl)- $N^{2}, N^{2}$-diethyl- $N^{1}$-(6-ethylbenzo[d] thiazol-2-yl)ethane-1,2-diamine (23). To a solution of $49(100 \mathrm{mg}, 0.24 \mathrm{mmol})$ in DMF/MeCN $(1: 1,2.4 \mathrm{~mL}, 0.1 \mathrm{M})$ was added $\mathrm{K}_{2} \mathrm{CO}_{3}(131$ $\mathrm{mg}, 0.95 \mathrm{mmol})$ and diethylamine $(247 \mu \mathrm{L}, 2.37 \mathrm{mmol})$. The reaction mixture was stirred at $50{ }^{\circ} \mathrm{C}$ for $18 \mathrm{~h}$ and then diluted with $\mathrm{H}_{2} \mathrm{O}$ and extracted with EtOAc. The organic layer was dried over $\mathrm{MgSO}_{4}$, 
filtered and evaporated in vacuo. The crude product was separated by silica gel column chromatography $\left(\mathrm{CH}_{2} \mathrm{Cl}_{2} / \mathrm{MeOH}, 15: 1, \mathrm{R}_{\mathrm{f}}=0.25\right)$ to produce the desired product 23 (68 $\mathrm{mg}, 69 \%$, yellow oil). ${ }^{1} \mathrm{H}$ NMR $\left(400 \mathrm{MHz}, \mathrm{CDCl}_{3}\right) \delta 7.30(\mathrm{~d}, J=8.0 \mathrm{~Hz}, 1 \mathrm{H}), 7.09-7.06(\mathrm{~m}, 1 \mathrm{H}), 6.99-6.97(\mathrm{~m}, 2 \mathrm{H}), 6.89(\mathrm{~d}, J=8.3$ Hz, 1H), 6.73-6.72 (m, 1H), $3.91(\mathrm{~s}, 3 \mathrm{H}), 3.84(\mathrm{~s}, 3 \mathrm{H}), 3.52$ (t, $J=7.1 \mathrm{~Hz}, 2 \mathrm{H}), 2.79(\mathrm{t}, J=7.1 \mathrm{~Hz}, 2 \mathrm{H})$, $2.58(\mathrm{q}, J=7.1 \mathrm{~Hz}, 4 \mathrm{H}), 2.48(\mathrm{q}, J=7.6 \mathrm{~Hz}, 2 \mathrm{H}), 1.09(\mathrm{t}, J=7.6 \mathrm{~Hz}, 3 \mathrm{H}), 1.02(\mathrm{t}, J=7.1 \mathrm{~Hz}, 6 \mathrm{H}) ;{ }^{13} \mathrm{C}$ $\operatorname{NMR}\left(100 \mathrm{MHz}, \mathrm{CDCl}_{3}\right) \delta 149.9,149.7,145.1,136.2,135.4,128.5,127.6,126.8,126.4,122.4,117.1$, $115.9,112.0,56.2,56.1,51.8,50.8,47.3(2 \mathrm{C}), 28.5,15.5,11.7(2 \mathrm{C})$; IR (neat) $v_{\max }=2966,2213,1584$, 1503, 1440, 1252, 1230, 1136, 1022, $729\left(\mathrm{~cm}^{-1}\right)$; HRMS (ESI): calcd. for $\mathrm{C}_{23} \mathrm{H}_{32} \mathrm{~N}_{3} \mathrm{O}_{2} \mathrm{~S}[\mathrm{M}+\mathrm{H}]^{+}$ 414.2210, found 414.2204.

\section{$N^{1}$-(3,4-Dimethoxyphenyl)- $N^{3}, N^{3}$-diethyl- $N^{1}$-(6-ethylbenzo $[d]$ thiazol-2-yl)propane-1,3-diamine}

(24). To a solution of $\mathbf{5 0}(100 \mathrm{mg}, 0.23 \mathrm{mmol})$ in $\mathrm{DMF} / \mathrm{MeCN}(1: 1,2.4 \mathrm{~mL}, 0.1 \mathrm{M})$ was added $\mathrm{K}_{2} \mathrm{CO}_{3}$ (127 mg, $0.92 \mathrm{mmol})$ and diethylamine $(240 \mu \mathrm{L}, 2.30 \mathrm{mmol})$. The reaction mixture was stirred at $50{ }^{\circ} \mathrm{C}$ for $12 \mathrm{~h}$ and then diluted with $\mathrm{H}_{2} \mathrm{O}$ and extracted with EtOAc. The organic layer was dried over $\mathrm{MgSO}_{4}$, filtered and evaporated in vacuo. The crude product was separated by silica gel column chromatography $\left(\mathrm{CH}_{2} \mathrm{Cl}_{2} / \mathrm{MeOH}, 20: 1, \mathrm{R}_{\mathrm{f}}=0.2\right)$ to produce the desired product $24\left(80.0 \mathrm{mg}, 81 \%\right.$, yellow oil). ${ }^{1} \mathrm{H}$ NMR $\left(400 \mathrm{MHz}, \mathrm{CDCl}_{3}\right) \delta 7.22(\mathrm{~d}, J=8.0 \mathrm{~Hz}, 1 \mathrm{H}), 7.08(\mathrm{dd}, J=8.3,2.1 \mathrm{~Hz}, 1 \mathrm{H}), 7.00-6.97(\mathrm{~m}, 2 \mathrm{H}), 6.89$ (d, $J=8.3 \mathrm{~Hz}, 1 \mathrm{H}), 6.71-6.70(\mathrm{~m}, 1 \mathrm{H}), 3.91(\mathrm{~s}, 3 \mathrm{H}), 3.85(\mathrm{~s}, 3 \mathrm{H}), 3.49(\mathrm{t}, J=7.3 \mathrm{~Hz}, 2 \mathrm{H}), 2.59-2.44$ $(\mathrm{m}, 8 \mathrm{H}), 1.96-1.88(\mathrm{~m}, 2 \mathrm{H}), 1.09(\mathrm{t}, J=7.6 \mathrm{~Hz}, 3 \mathrm{H}), 1.01(\mathrm{t}, J=7.1 \mathrm{~Hz}, 6 \mathrm{H}) ;{ }^{13} \mathrm{C}$ NMR $(100 \mathrm{MHz}$ $\left.\mathrm{CDCl}_{3}\right) \delta 150.0,149.8,145.3,136.6,135.1,128.4,127.8,126.4,126.3,122.2,117.3,115.6,112.0,56.2$, $56.1,52.4,49.8,46.8$ (2C), 29.8, 28.6, 25.3, 15.5, 11.3; IR (neat) $v_{\max }=2987,2213,1584,1503,1441$, 1253, 1231, 1136, 1023, 806, $734\left(\mathrm{~cm}^{-1}\right)$; HRMS (ESI): calcd. for $\mathrm{C}_{24} \mathrm{H}_{34} \mathrm{~N}_{3} \mathrm{O}_{2} \mathrm{~S}[\mathrm{M}+\mathrm{H}]^{+} 428.2366$, found 428.2369 .

\section{$N^{1}$-(3,4-Dimethoxyphenyl)- $N^{4}, N^{4}$-diethyl- $N^{1}$-(6-ethylbenzo[d]thiazol-2-yl)butane-1,4-diamine}

(25). To a solution of 51 (134 mg, $0.30 \mathrm{mmol})$ in $\mathrm{DMF} / \mathrm{MeCN}(1: 1,3.0 \mathrm{~mL}, 0.1 \mathrm{M})$ was added $\mathrm{K}_{2} \mathrm{CO}_{3}$ (165 mg, $1.19 \mathrm{mmol})$ and diethylamine $(310 \mu \mathrm{L}, 2.98 \mathrm{mmol})$. The reaction mixture was stirred at $50{ }^{\circ} \mathrm{C}$ for $15 \mathrm{~h}$ and then diluted with $\mathrm{H}_{2} \mathrm{O}$ and extracted with EtOAc. The organic layer was dried over $\mathrm{MgSO}_{4}$, 
filtered and evaporated in vacuo. The crude product was separated by silica gel column chromatography $\left(\mathrm{CH}_{2} \mathrm{Cl}_{2} / \mathrm{MeOH}, 15: 1, \mathrm{R}_{\mathrm{f}}=0.2\right)$ to produce the desired product $25\left(108 \mathrm{mg}, 82 \%\right.$, yellow oil). ${ }^{1} \mathrm{H}$ NMR $\left(400 \mathrm{MHz}, \mathrm{CDCl}_{3}\right) \delta 7.22(\mathrm{~d}, J=8.0 \mathrm{~Hz}, 1 \mathrm{H}), 7.08(\mathrm{dd}, J=8.3,2.1 \mathrm{~Hz}, 1 \mathrm{H}), 6.99-6.97(\mathrm{~m}, 2 \mathrm{H}), 6.89$ (d, $J=8.3 \mathrm{~Hz}, 1 \mathrm{H}), 6.70-6.69(\mathrm{~m}, 1 \mathrm{H}), 3.91(\mathrm{~s}, 3 \mathrm{H}), 3.85(\mathrm{~s}, 3 \mathrm{H}), 3.45(\mathrm{t}, J=7.4 \mathrm{~Hz}, 2 \mathrm{H}), 2.55(\mathrm{q}, J=$ $7.2 \mathrm{~Hz}, 4 \mathrm{H}), 2.51-2.44(\mathrm{~m}, 4 \mathrm{H}), 1.82-1.74(\mathrm{~m}, 2 \mathrm{H}), 1.65-1.58(\mathrm{~m}, 2 \mathrm{H}), 1.08(\mathrm{t}, J=7.6 \mathrm{~Hz}, 3 \mathrm{H}), 1.03$ $(\mathrm{t}, J=7.2 \mathrm{~Hz}, 6 \mathrm{H}) ;{ }^{13} \mathrm{C}$ NMR $\left(100 \mathrm{MHz}, \mathrm{CDCl}_{3}\right) \delta 150.0,149.8,145.2,136.6,135.1,128.4,127.8$, $126.42,126.36,122.3,117.3,115.7,112.0,56.2,56.1,54.0,52.4,46.9(2 \mathrm{C}), 28.5,26.1,23.9,15.5,11.6$ (2C); IR (neat) $v_{\max }=2966,2213,1584,1503,1441,1398,1253,1231,1138,1023,762\left(\mathrm{~cm}^{-1}\right)$; HRMS (ESI): calcd. for $\mathrm{C}_{25} \mathrm{H}_{36} \mathrm{~N}_{3} \mathrm{O}_{2} \mathrm{~S}[\mathrm{M}+\mathrm{H}]^{+} 442.2523$, found 442.2519 .

\section{$N^{1}$-(3,4-Dimethoxyphenyl)- $N^{5}, N^{5}$-diethyl- $N^{1}$-(6-ethylbenzo[ $\left.d\right]$ thiazol-2-yl)pentane-1,5-diamine}

(26). To a solution of $52(150 \mathrm{mg}, 0.32 \mathrm{mmol})$ in $\mathrm{DMF} / \mathrm{MeCN}(1: 1,3.2 \mathrm{~mL}, 0.1 \mathrm{M})$ was added $\mathrm{K}_{2} \mathrm{CO}_{3}$ (179 mg, $1.30 \mathrm{mmol})$ and diethylamine $(335 \mu \mathrm{L}, 3.24 \mathrm{mmol})$. The reaction mixture was stirred at $50{ }^{\circ} \mathrm{C}$ for $13 \mathrm{~h}$ and then diluted with $\mathrm{H}_{2} \mathrm{O}$ and extracted with EtOAc. The organic layer was dried over $\mathrm{MgSO}_{4}$, filtered and evaporated in vacuo. The crude product was separated by silica gel column chromatography $\left(\mathrm{CH}_{2} \mathrm{Cl}_{2} / \mathrm{MeOH}, 10: 1, \mathrm{R}_{\mathrm{f}}=0.25\right)$ to produce the desired product $\mathbf{2 6}\left(119 \mathrm{mg}, 80 \%\right.$, yellow oil). ${ }^{1} \mathrm{H}$ NMR $\left(400 \mathrm{MHz}, \mathrm{CDCl}_{3}\right) \delta 7.22(\mathrm{~d}, J=8.0 \mathrm{~Hz}, 1 \mathrm{H}), 7.09(\mathrm{dd}, J=8.3,2.1 \mathrm{~Hz}, 1 \mathrm{H}), 7.00-6.97(\mathrm{~m}, 2 \mathrm{H}), 6.90$ $(\mathrm{d}, J=8.3 \mathrm{~Hz}, 1 \mathrm{H}), 6.71(\mathrm{~d}, J=2.0 \mathrm{~Hz}, 1 \mathrm{H}), 3.92(\mathrm{~s}, 3 \mathrm{H}), 3.86(\mathrm{~s}, 3 \mathrm{H}), 3.43(\mathrm{t}, J=7.4 \mathrm{~Hz}, 2 \mathrm{H}), 2.62-$ $2.57(\mathrm{~m}, 4 \mathrm{H}), 2.51-2.45(\mathrm{~m}, 4 \mathrm{H}), 1.85-1.77(\mathrm{~m}, 2 \mathrm{H}), 1.60-1.52(\mathrm{~m}, 2 \mathrm{H}), 1.49-1.41(\mathrm{~m}, 2 \mathrm{H}), 1.11-1.04$ $(\mathrm{m}, 9 \mathrm{H}) ;{ }^{13} \mathrm{C} \mathrm{NMR}\left(100 \mathrm{MHz}, \mathrm{CDCl}_{3}\right) \delta 150.0,149.8,145.3,136.6,135.1,128.3,127.8,126.4(2 \mathrm{C})$, $122.2,117.3,115.7,112.1,56.2,56.1,53.8,52.4,46.8(2 \mathrm{C}), 28.5,27.8,25.7,24.4,15.5,10.7$ (2C); IR (neat) $v_{\max }=2967,2213,1738,1584,1503,1253,1231,1137,1023,762\left(\mathrm{~cm}^{-1}\right)$; HRMS (ESI): calcd. for $\mathrm{C}_{26} \mathrm{H}_{38} \mathrm{~N}_{3} \mathrm{O}_{2} \mathrm{~S}[\mathrm{M}+\mathrm{H}]^{+} 456.2679$, found 456.2675.

\section{$N^{1}$-Cyclohexyl- $N^{2}$-(3,4-dimethoxyphenyl)- $N^{2}$-(6-ethylbenzo[d]thiazol-2-yl)ethane-1,2-diamine}

(27). To a solution of 49 (100 mg, $0.24 \mathrm{mmol})$ in $\mathrm{DMF} / \mathrm{MeCN}(1: 1,2.4 \mathrm{~mL}, 0.1 \mathrm{M})$ was added $\mathrm{K}_{2} \mathrm{CO}_{3}$ (131 mg, $0.95 \mathrm{mmol})$ and cyclohexylamine $(272 \mu \mathrm{L}, 2.37 \mathrm{mmol})$. The reaction mixture was stirred at $50{ }^{\circ} \mathrm{C}$ for $13 \mathrm{~h}$ and then diluted with $\mathrm{H}_{2} \mathrm{O}$ and extracted with EtOAc. The organic layer was dried over 
$\mathrm{MgSO}_{4}$, filtered and evaporated in vacuo. The crude product was separated by silica gel column chromatography $\left(\mathrm{CH}_{2} \mathrm{Cl}_{2} / \mathrm{MeOH}, 10: 1, \mathrm{R}_{\mathrm{f}}=0.2\right)$ to produce the desired product $27(99.0 \mathrm{mg}, 95 \%$, ivory solid). ${ }^{1} \mathrm{H}$ NMR $\left(400 \mathrm{MHz}, \mathrm{CDCl}_{3}\right) \delta 7.11(\mathrm{~d}, J=8.0 \mathrm{~Hz}, 1 \mathrm{H}), 7.07-7.04(\mathrm{~m}, 1 \mathrm{H}), 6.99-6.96(\mathrm{~m}$, 2H), $6.86(\mathrm{~d}, J=8.3 \mathrm{~Hz}, 1 \mathrm{H}), 6.68-6.67(\mathrm{~m}, 1 \mathrm{H}), 4.10-3.95(\mathrm{~m}, 3 \mathrm{H}), 3.88(\mathrm{~s}, 3 \mathrm{H}), 3.82(\mathrm{~s}, 3 \mathrm{H}), 3.70-$ $3.69(\mathrm{~m}, 2 \mathrm{H}), 3.45(\mathrm{t}, J=7.7 \mathrm{~Hz}, 2 \mathrm{H}), 2.46(\mathrm{q}, J=7.6 \mathrm{~Hz}, 2 \mathrm{H}), 1.89-1.87(\mathrm{~m}, 2 \mathrm{H}), 1.79-1.76(\mathrm{~m}, 2 \mathrm{H})$, $1.66-1.63(\mathrm{~m}, 1 \mathrm{H}), 1.52-1.30(\mathrm{~m}, 4 \mathrm{H}), 1.08(\mathrm{t}, J=7.6 \mathrm{~Hz}, 3 \mathrm{H}) ;{ }^{13} \mathrm{C} \mathrm{NMR}\left(100 \mathrm{MHz}, \mathrm{CDCl}_{3}\right) \delta 158.5$, $149.9,149.6,145.4,139.7,133.2,128.3,127.9,127.4,126.4,122.1,117.4,111.9,56.2,56.0,53.1(2 \mathrm{C})$, $47.4,40.6,29.8,28.6,25.7,25.4$ (2C), 15.4; IR (neat) $v_{\max }=3315,2931,1655,1549,1503,1253,1230$, 1136, 1020, $729\left(\mathrm{~cm}^{-1}\right)$; HRMS (ESI): calcd. for $\mathrm{C}_{25} \mathrm{H}_{34} \mathrm{~N}_{3} \mathrm{O}_{2} \mathrm{~S}[\mathrm{M}+\mathrm{H}]^{+} 440.2366$, found 440.2362.

\section{$N^{1}$-Cyclohexyl- $N^{3}$-(3,4-dimethoxyphenyl)- $N^{3}$-(6-ethylbenzo[d] thiazol-2-yl)propane-1,3-diamine}

(28). To a solution of $\mathbf{5 0}(150 \mathrm{mg}, 0.34 \mathrm{mmol})$ in $\mathrm{DMF} / \mathrm{MeCN}(1: 1,3.0 \mathrm{~mL}, 0.1 \mathrm{M})$ was added $\mathrm{K}_{2} \mathrm{CO}_{3}$ (190 mg, $1.38 \mathrm{mmol})$ and cyclohexylamine $(390 \mu \mathrm{L}, 3.40 \mathrm{mmol})$. The reaction mixture was stirred at $50{ }^{\circ} \mathrm{C}$ for $14 \mathrm{~h}$ and then diluted with $\mathrm{H}_{2} \mathrm{O}$ and extracted with EtOAc. The organic layer was dried over $\mathrm{MgSO}_{4}$, filtered and evaporated in vacuo. The crude product was separated by silica gel column chromatography $\left(\mathrm{CH}_{2} \mathrm{Cl}_{2} / \mathrm{MeOH}, 15: 1, \mathrm{R}_{\mathrm{f}}=0.2\right)$ to produce the desired product $\mathbf{2 8}(83 \mathrm{mg}, 54 \%$, ivory solid). ${ }^{1} \mathrm{H}$ NMR (400 MHz, $\left.\mathrm{CDCl}_{3}\right) \delta 7.12-7.09(\mathrm{~m}, 2 \mathrm{H}), 7.03-7.00(\mathrm{~m}, 2 \mathrm{H}), 6.94(\mathrm{~d}, J=8.4 \mathrm{~Hz}, 1 \mathrm{H})$, 6.60-6.59 (m, 1H), $4.52(\mathrm{tt}, J=11.6,3.5 \mathrm{~Hz}, 1 \mathrm{H}), 3.93(\mathrm{~s}, 3 \mathrm{H}), 3.88(\mathrm{~s}, 3 \mathrm{H}), 3.68-3.62(\mathrm{~m}, 1 \mathrm{H}), 3.55-$ $3.41(\mathrm{~m}, 3 \mathrm{H}), 2.48(\mathrm{q}, J=7.6 \mathrm{~Hz}, 2 \mathrm{H}), 2.28-2.14(\mathrm{~m}, 2 \mathrm{H}), 1.96-1.69(\mathrm{~m}, 8 \mathrm{H}), 1.54-1.43(\mathrm{~m}, 2 \mathrm{H}), 1.10$ $(\mathrm{t}, J=7.5 \mathrm{~Hz}, 3 \mathrm{H}) ;{ }^{13} \mathrm{C} \mathrm{NMR}\left(100 \mathrm{MHz}, \mathrm{CDCl}_{3}\right) \delta 152.4,150.7,150.0,147.7,139.5,132.1,129.1$, $128.6,127.3,126.9,119.2,118.1,112.3,58.1,56.5,56.1,48.0,39.7,30.14,30.09,28.8,25.4,24.9,24.8$, $21.8,15.4$; IR (neat) $v_{\max }=3453,2932,1583,1503,1321,1253,1231,1137,1020,725\left(\mathrm{~cm}^{-1}\right)$; HRMS (ESI): calcd. for $\mathrm{C}_{26} \mathrm{H}_{36} \mathrm{~N}_{3} \mathrm{O}_{2} \mathrm{~S}[\mathrm{M}+\mathrm{H}]^{+} 454.2523$, found 454.2517 .

\section{$N^{1}$-Cyclohexyl- $N^{4}$-(3,4-dimethoxyphenyl)- $N^{4}$-(6-ethylbenzo $[d]$ thiazol-2-yl)butane-1,4-diamine}

(29). To a solution of $51(150 \mathrm{mg}, 0.33 \mathrm{mmol})$ in $\mathrm{DMF} / \mathrm{MeCN}(1: 1,3.4 \mathrm{~mL}, 0.1 \mathrm{M})$ was added $\mathrm{K}_{2} \mathrm{CO}_{3}$ (185 mg, $1.34 \mathrm{mmol})$ and cyclohexylamine $(383 \mu \mathrm{L}, 3.34 \mathrm{mmol})$. The reaction mixture was stirred at $50{ }^{\circ} \mathrm{C}$ for $13 \mathrm{~h}$ and then diluted with $\mathrm{H}_{2} \mathrm{O}$ and extracted with EtOAc. The organic layer was dried over 
$\mathrm{MgSO}_{4}$, filtered and evaporated in vacuo. The crude product was separated by silica gel column chromatography $\left(\mathrm{CH}_{2} \mathrm{Cl}_{2} / \mathrm{MeOH}, 15: 1, \mathrm{R}_{\mathrm{f}}=0.2\right)$ to produce the desired product $29(91.0 \mathrm{mg}, 58 \%$, white solid). ${ }^{1} \mathrm{H}$ NMR $\left(400 \mathrm{MHz}, \mathrm{CDCl}_{3}\right) \delta 7.21(\mathrm{~d}, J=8.0 \mathrm{~Hz}, 1 \mathrm{H}), 7.08(\mathrm{dd}, J=8.3,2.0 \mathrm{~Hz}, 1 \mathrm{H})$, 6.99-6.96 (m, 2H), $6.89(\mathrm{~d}, J=8.3 \mathrm{~Hz}, 1 \mathrm{H}), 6.70-6.69(\mathrm{~m}, 1 \mathrm{H}), 3.91(\mathrm{~s}, 3 \mathrm{H}), 3.84(\mathrm{~s}, 3 \mathrm{H}), 3.43$ (t, $J=$ $7.4 \mathrm{~Hz}, 2 \mathrm{H}), 2.69(\mathrm{t}, J=7.3 \mathrm{~Hz}, 2 \mathrm{H}), 2.50-2.44(\mathrm{~m}, 3 \mathrm{H}), 1.90-1.57(\mathrm{~m}, 10 \mathrm{H}), 1.28-1.05(\mathrm{~m}, 8 \mathrm{H}) ;{ }^{13} \mathrm{C}$ $\operatorname{NMR}\left(100 \mathrm{MHz}, \mathrm{CDCl}_{3}\right) \delta 150.0,149.7,145.2,136.6,135.1,128.3,127.8,126.41,126.35,122.2,117.3$, 115.7, 112.0, 57.0, 56.2, 56.0, 53.9 (2C), 46.2, 33.1, 28.5, 26.9, 26.1, 25.9, 25.1 (2C), 15.5; IR (neat) $v_{\max }=2929,2213,1585,1503,1442,1253,1230,1136,1023,728\left(\mathrm{~cm}^{-1}\right)$; HRMS (ESI): calcd. for $\mathrm{C}_{27} \mathrm{H}_{38} \mathrm{~N}_{3} \mathrm{O}_{2} \mathrm{~S}[\mathrm{M}+\mathrm{H}]^{+}$468.2679, found 468.2675.

\section{$N^{1}$-Cyclohexyl- $N^{5}$-(3,4-dimethoxyphenyl)- $N^{5}$-(6-ethylbenzo $[d]$ thiazol-2-yl)pentane-1,5-diamine}

(30). To a solution of $52(150 \mathrm{mg}, 0.32 \mathrm{mmol})$ in $\mathrm{DMF} / \mathrm{MeCN}(1: 1,3.2 \mathrm{~mL}, 0.1 \mathrm{M})$ was added $\mathrm{K}_{2} \mathrm{CO}_{3}$ (179 mg, $1.30 \mathrm{mmol})$ and cyclohexylamine $(371 \mu \mathrm{L}, 3.24 \mathrm{mmol})$. The reaction mixture was stirred at $50{ }^{\circ} \mathrm{C}$ for $16 \mathrm{~h}$ and then diluted with $\mathrm{H}_{2} \mathrm{O}$ and extracted with EtOAc. The organic layer was dried over $\mathrm{MgSO}_{4}$, filtered and evaporated in vacuo. The crude product was separated by silica gel column chromatography $\left(\mathrm{CH}_{2} \mathrm{Cl}_{2} / \mathrm{MeOH}, 15: 1, \mathrm{R}_{\mathrm{f}}=0.2\right)$ to produce the desired product 30 (112 $\mathrm{mg}, 72 \%$, yellow oil). ${ }^{1} \mathrm{H}$ NMR (400 MHz, $\left.\mathrm{CDCl}_{3}\right) \delta 7.19(\mathrm{~d}, J=8.0 \mathrm{~Hz}, 1 \mathrm{H}), 7.07(\mathrm{dd}, J=8.3,2.1 \mathrm{~Hz}, 1 \mathrm{H})$, 6.99-6.95 (m, 2H), $6.88(\mathrm{~d}, J=8.3 \mathrm{~Hz}, 1 \mathrm{H}), 6.69-6.68(\mathrm{~m}, 1 \mathrm{H}), 3.90(\mathrm{~s}, 3 \mathrm{H}), 3.84(\mathrm{~s}, 3 \mathrm{H}), 3.41(\mathrm{t}, J=$ $7.4 \mathrm{~Hz}, 2 \mathrm{H}), 2.69$ (t, $J=7.5 \mathrm{~Hz}, 2 \mathrm{H}), 2.53-2.43(\mathrm{~m}, 3 \mathrm{H}), 1.94-1.90(\mathrm{~m}, 2 \mathrm{H}), 1.82-1.70(\mathrm{~m}, 4 \mathrm{H}), 1.66-$ $1.58(\mathrm{~m}, 3 \mathrm{H}), 1.51-1.45(\mathrm{~m}, 2 \mathrm{H}), 1.25-1.11(\mathrm{~m}, 5 \mathrm{H}), 1.07(\mathrm{t}, J=7.6 \mathrm{~Hz}, 3 \mathrm{H}) ;{ }^{13} \mathrm{C}$ NMR $(100 \mathrm{MHz}$, $\left.\mathrm{CDCl}_{3}\right) \delta 150.0,149.7,145.2,136.6,135.1,128.3,127.8,126.4,126.3,122.2,117.3,115.7,112.0,57.0$ $56.2,56.1,53.9$ (2C), 46.1, 32.6, 29.0, 28.5, 27.8, 25.9, 25.0 (2C), 24.3, 15.4; IR (neat) $v_{\max }=2929$, $2213,1584,1503,1321,1253,1231,1023,729\left(\mathrm{~cm}^{-1}\right)$; HRMS (ESI): calcd. for $\mathrm{C}_{28} \mathrm{H}_{40} \mathrm{~N}_{3} \mathrm{O}_{2} \mathrm{~S}[\mathrm{M}+\mathrm{H}]^{+}$ 482.2836, found 482.2833 .

\section{$N^{1}$-Cyclopentyl- $N^{2}$-(3,4-dimethoxyphenyl)- $N^{2}$-(6-ethylbenzo $[d]$ thiazol-2-yl)ethane-1,2-diamine}

(31). To a solution of $49(1.0 \mathrm{~g}, 2.37 \mathrm{mmol})$ in $\mathrm{DMF} / \mathrm{MeCN}(1: 1,24 \mathrm{~mL}, 0.1 \mathrm{M})$ was added $\mathrm{K}_{2} \mathrm{CO}_{3}$ $(1.31 \mathrm{~g}, 9.49 \mathrm{mmol})$ and cyclopentylamine $(2.3 \mathrm{~mL}, 23.7 \mathrm{mmol})$. The reaction mixture was stirred at 
$50{ }^{\circ} \mathrm{C}$ for $13 \mathrm{~h}$ and then diluted with $\mathrm{H}_{2} \mathrm{O}$ and extracted with EtOAc. The organic layer was dried over $\mathrm{MgSO}_{4}$, filtered and evaporated in vacuo. The crude product was separated by silica gel column chromatography $\left(\mathrm{CH}_{2} \mathrm{Cl}_{2} / \mathrm{MeOH}, 15: 1, \mathrm{R}_{\mathrm{f}}=0.2\right)$ to produce the desired product 31 ( $767 \mathrm{mg}, 76 \%$, white solid). ${ }^{1} \mathrm{H}$ NMR (400 MHz, $\left.\mathrm{CD}_{2} \mathrm{Cl}_{2}\right) \delta 7.13(\mathrm{~d}, J=8.0 \mathrm{~Hz}, 1 \mathrm{H}), 7.09$ (dd, $\left.J=8.3,2.1 \mathrm{~Hz}, 1 \mathrm{H}\right), 7.02-$ $7.00(\mathrm{~m}, 2 \mathrm{H}), 6.90(\mathrm{~d}, J=8.3 \mathrm{~Hz}, 1 \mathrm{H}), 6.75-6.74(\mathrm{~m}, 1 \mathrm{H}), 4.38-4.31(\mathrm{~m}, 1 \mathrm{H}), 3.86(\mathrm{~s}, 3 \mathrm{H}), 3.79(\mathrm{~s}$, $3 \mathrm{H}), 3.72-3.61(\mathrm{~m}, 3 \mathrm{H}), 3.36(\mathrm{t}, J=7.2 \mathrm{~Hz}, 2 \mathrm{H}), 2.49$ (q, $J=7.6 \mathrm{~Hz}, 2 \mathrm{H}), 1.90-1.84(\mathrm{~m}, 2 \mathrm{H}), 1.74$ $1.54(\mathrm{~m}, 6 \mathrm{H}), 1.10(\mathrm{t}, J=7.6 \mathrm{~Hz}, 3 \mathrm{H}) ;{ }^{13} \mathrm{C} \mathrm{NMR}\left(100 \mathrm{MHz}, \mathrm{CDCl}_{3}\right) \delta 150.0,149.7,145.1,136.4,135.2$, $128.4,127.6,127.1,126.3,122.5,117.2,116.0,112.0,56.24,56.21,56.1,54.7(2 \mathrm{C}), 51.0,28.5,26.0$ (2C), 24.4, 15.5; IR (neat) $v_{\max }=3173,2961,1658,1547,1498,1253,1228,1136,1019,804\left(\mathrm{~cm}^{-1}\right)$; HRMS (FAB): calcd. for $\mathrm{C}_{24} \mathrm{H}_{32} \mathrm{~N}_{3} \mathrm{O}_{2} \mathrm{~S}[\mathrm{M}+\mathrm{H}]^{+}$426.2215, found 426.2214.

\section{$N^{1}$-Cyclopentyl- $N^{3}$-(3,4-dimethoxyphenyl)- $N^{3}$-(6-ethylbenzo $[d]$ thiazol-2-yl)propane-1,3-diamine}

(32). To a solution of $\mathbf{5 0}(150 \mathrm{mg}, 0.34 \mathrm{mmol})$ in $\mathrm{DMF} / \mathrm{MeCN}(1: 1,3.4 \mathrm{~mL}, 0.1 \mathrm{M})$ was added $\mathrm{K}_{2} \mathrm{CO}_{3}$ (188 mg, $1.36 \mathrm{mmol}$ ) and cyclopentylamine $(337 \mu \mathrm{L}, 3.40 \mathrm{mmol})$. The reaction mixture was stirred at $50{ }^{\circ} \mathrm{C}$ for $16 \mathrm{~h}$ and then diluted with $\mathrm{H}_{2} \mathrm{O}$ and extracted with EtOAc. The organic layer was dried over $\mathrm{MgSO}_{4}$, filtered and evaporated in vacuo. The crude product was separated by silica gel column chromatography $\left(\mathrm{CH}_{2} \mathrm{Cl}_{2} / \mathrm{MeOH}, 15: 1, \mathrm{R}_{\mathrm{f}}=0.2\right)$ to produce the desired product $32(91.0 \mathrm{mg}, 61 \%$, ivory solid). ${ }^{1} \mathrm{H}$ NMR (400 MHz, $\left.\mathrm{CDCl}_{3}\right) \delta 7.13-7.08(\mathrm{~m}, 2 \mathrm{H}), 7.02-6.99(\mathrm{~m}, 2 \mathrm{H}), 6.94$ (d, J=8.4 Hz, $1 \mathrm{H}), 6.60-6.59(\mathrm{~m}, 1 \mathrm{H}), 5.02-4.94(\mathrm{~m}, 1 \mathrm{H}), 3.93(\mathrm{~s}, 3 \mathrm{H}), 3.88(\mathrm{~s}, 3 \mathrm{H}), 3.70-3.64(\mathrm{~m}, 1 \mathrm{H}), 3.54-3.48$ (m, 1H), $3.43(\mathrm{t}, J=5.8 \mathrm{~Hz}, 2 \mathrm{H}), 2.48(\mathrm{q}, J=7.6 \mathrm{~Hz}, 2 \mathrm{H}), 2.32-2.13(\mathrm{~m}, 4 \mathrm{H}), 1.86-1.50(\mathrm{~m}, 7 \mathrm{H}), 1.09$ $(\mathrm{t}, J=7.6 \mathrm{~Hz}, 3 \mathrm{H}) ;{ }^{13} \mathrm{C} \mathrm{NMR}\left(100 \mathrm{MHz}, \mathrm{CDCl}_{3}\right) \delta 152.9,150.7,150.0,147.7,139.6,132.2,129.1$, $128.6,127.2,126.8,119.3,118.1,112.3,60.0,56.4,56.1,47.9,40.0,29.2,28.8,28.7,24.3,24.2,21.9$, 15.4; IR (neat) $v_{\max }=3320,2921,1639,1583,1503,1442,1253,1231,1021,729\left(\mathrm{~cm}^{-1}\right) ;$ HRMS (ESI): calcd. for $\mathrm{C}_{25} \mathrm{H}_{34} \mathrm{~N}_{3} \mathrm{O}_{2} \mathrm{~S}[\mathrm{M}+\mathrm{H}]^{+} 440.2366$, found 440.2373 .

\section{$N^{1}$-Cyclopentyl- $N^{4}$-(3,4-dimethoxyphenyl)- $N^{4}$-(6-ethylbenzo[ $\left.d\right]$ thiazol-2-yl)butane-1,4-diamine}

(33). To a solution of 51 (375 mg, $0.84 \mathrm{mmol})$ in $\mathrm{DMF} / \mathrm{MeCN}(1: 1,8.4 \mathrm{~mL}, 0.1 \mathrm{M})$ was added $\mathrm{K}_{2} \mathrm{CO}_{3}$ (462 mg, $3.34 \mathrm{mmol}$ ) and cyclopentylamine $(827 \mu \mathrm{L}, 8.35 \mathrm{mmol}$ ). The reaction mixture was stirred at 
$50{ }^{\circ} \mathrm{C}$ for $16 \mathrm{~h}$ and then diluted with $\mathrm{H}_{2} \mathrm{O}$ and extracted with EtOAc. The organic layer was dried over $\mathrm{MgSO}_{4}$, filtered and evaporated in vacuo. The crude product was separated by silica gel column chromatography $\left(\mathrm{CH}_{2} \mathrm{Cl}_{2} / \mathrm{MeOH}, 10: 1, \mathrm{R}_{\mathrm{f}}=0.2\right)$ to produce the desired product $33(140 \mathrm{mg}, 37 \%$, colorless oil). ${ }^{1} \mathrm{H}$ NMR (400 MHz, $\left.\mathrm{CDCl}_{3}\right) \delta 7.20(\mathrm{~d}, J=8.0 \mathrm{~Hz}, 1 \mathrm{H}), 7.06(\mathrm{dd}, J=8.3,2.1 \mathrm{~Hz}, 1 \mathrm{H})$, 6.99-6.95 (m, 2H), $6.88(\mathrm{~d}, J=8.3 \mathrm{~Hz}, 1 \mathrm{H}), 6.67-6.66(\mathrm{~m}, 1 \mathrm{H}), 3.90(\mathrm{~s}, 3 \mathrm{H}), 3.83(\mathrm{~s}, 3 \mathrm{H}), 3.43(\mathrm{t}, J=$ $7.3 \mathrm{~Hz}, 2 \mathrm{H}), 3.38-3.31(\mathrm{~m}, 1 \mathrm{H}), 2.92(\mathrm{t}, J=8.0 \mathrm{~Hz}, 2 \mathrm{H}), 2.45(\mathrm{q}, J=7.6 \mathrm{~Hz}, 2 \mathrm{H}), 2.05-1.97(\mathrm{~m}, 4 \mathrm{H})$, $1.92(\mathrm{~s}, 1 \mathrm{H}), 1.87-1.79(\mathrm{~m}, 6 \mathrm{H}), 1.58-1.49(\mathrm{~m}, 2 \mathrm{H}), 1.06(\mathrm{t}, J=7.6 \mathrm{~Hz}, 3 \mathrm{H}) ;{ }^{13} \mathrm{C}$ NMR $(100 \mathrm{MHz}$, $\left.\mathrm{CDCl}_{3}\right) \delta 150.1,149.8,145.5,136.7,134.6,128.3,127.9,126.54,126.49,121.9,117.3,115.5,112.1$, $59.3,56.3,56.1,53.2,46.4,29.8(2 \mathrm{C}), 28.5,25.5,23.9(2 \mathrm{C}), 23.0,15.5$; IR (neat) $v_{\max }=3401,2958$, 2212, 1583, 1502, 1440, 1252, 1230, 1135, 1021, $762\left(\mathrm{~cm}^{-1}\right)$; HRMS (FAB): calcd. for $\mathrm{C}_{26} \mathrm{H}_{36} \mathrm{~N}_{3} \mathrm{O}_{2} \mathrm{~S}$ $[\mathrm{M}+\mathrm{H}]^{+} 454.2528$, found 454.2527 .

\section{$N^{1}$-Cyclopentyl- $N^{5}$-(3,4-dimethoxyphenyl)- $N^{5}$-(6-ethylbenzo $[d]$ thiazol-2-yl)pentane-1,5-diamine}

(34). To a solution of $\mathbf{5 2}(150 \mathrm{mg}, 0.32 \mathrm{mmol})$ in $\mathrm{DMF} / \mathrm{MeCN}(1: 1,3.2 \mathrm{~mL}, 0.1 \mathrm{M})$ was added $\mathrm{K}_{2} \mathrm{CO}_{3}$ (179 $\mathrm{mg}, 1.30 \mathrm{mmol})$ and cyclopentylamine $(320 \mu \mathrm{L}, 3.24 \mathrm{mmol})$. The reaction mixture was stirred at $50{ }^{\circ} \mathrm{C}$ for $14 \mathrm{~h}$ and then diluted with $\mathrm{H}_{2} \mathrm{O}$ and extracted with EtOAc. The organic layer was dried over $\mathrm{MgSO}_{4}$, filtered and evaporated in vacuo. The crude product was separated by silica gel column chromatography $\left(\mathrm{CH}_{2} \mathrm{Cl}_{2} / \mathrm{MeOH}, 15: 1, \mathrm{R}_{\mathrm{f}}=0.2\right)$ to produce the desired product 34 (136 $\mathrm{mg}, 89 \%$, yellow oil). ${ }^{1} \mathrm{H}$ NMR (400 MHz, $\left.\mathrm{CDCl}_{3}\right) \delta 7.20(\mathrm{~d}, J=8.0 \mathrm{~Hz}, 1 \mathrm{H}), 7.09-7.06(\mathrm{~m}, 1 \mathrm{H}), 6.99-6.96(\mathrm{~m}$, 2H), $6.89(\mathrm{~d}, J=8.3 \mathrm{~Hz}, 1 \mathrm{H}), 6.69-6.68(\mathrm{~m}, 1 \mathrm{H}), 3.90(\mathrm{~s}, 3 \mathrm{H}), 3.84(\mathrm{~s}, 3 \mathrm{H}), 3.41(\mathrm{t}, J=7.4 \mathrm{~Hz}, 2 \mathrm{H})$, 3.10-3.03 (m, 1H), $2.61(\mathrm{t}, J=7.2 \mathrm{~Hz}, 2 \mathrm{H}), 2.47(\mathrm{q}, J=7.6 \mathrm{~Hz}, 2 \mathrm{H}), 1.90-1.74(\mathrm{~m}, 4 \mathrm{H}), 1.72-1.62(\mathrm{~m}$, 2H), 1.61-1.43 (m, 6H), 1.39-1.31 (m, 2H), $1.08(\mathrm{t}, J=7.6 \mathrm{~Hz}, 3 \mathrm{H}) ;{ }^{13} \mathrm{C}$ NMR $\left(100 \mathrm{MHz}, \mathrm{CDCl}_{3}\right) \delta$ $149.9,149.7,145.1,136.6,135.1,128.3,127.7,126.33,126.27122 .2,117.2,115.7,112.0,59.9,56.1$, $56.0,53.9,48.3,32.8(2 \mathrm{C}), 29.5,28.4,27.8,24.3,24.1(2 \mathrm{C}), 15.4$; IR (neat) $v_{\max }=2937,2213,1585$, 1503, 1321, 1253, 1231, 1023, $728\left(\mathrm{~cm}^{-1}\right)$; HRMS (ESI): calcd. for $\mathrm{C}_{27} \mathrm{H}_{38} \mathrm{~N}_{3} \mathrm{O}_{2} \mathrm{~S}[\mathrm{M}+\mathrm{H}]^{+} 468.2679$, found 468.2697 . 
(4) Synthesis of Straight-Chain Alkyl Analogs 12-14

\section{A. Synthetic Procedures for the Preparation of 53-55}

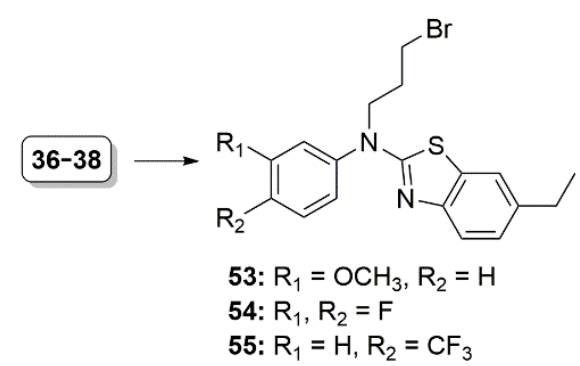

$N$-(3-Bromopropyl)-6-ethyl- $N$-(3-methoxyphenyl)benzo[d]thiazol-2-amine (53). To a solution of compound 36 (200 mg, $0.70 \mathrm{mmol})$ in dry DMF $(3.5 \mathrm{~mL}, 0.2 \mathrm{M})$ was added $\mathrm{NaH}(60 \%$ in mineral oil, $70 \mathrm{mg}, 1.76 \mathrm{mmol}$ ), and the mixture was stirred at $0{ }^{\circ} \mathrm{C}$ for $10 \mathrm{~min}$. To this reaction mixture was added 1,3-dibromo-propane $(717 \mu \mathrm{L}, 7.03 \mathrm{mmol})$ and then the mixture was allowed to warm to room temperature slowly and stirred at $50{ }^{\circ} \mathrm{C}$ for $14 \mathrm{~h}$. The reaction mixture was diluted with $\mathrm{H}_{2} \mathrm{O}$ and extracted with EtOAc. The organic layer was dried over $\mathrm{MgSO}_{4}$, filtered and evaporated in vacuo. The crude product was separated by silica gel column chromatography (hexane/ether, $2.5: 1, R_{f}=0.2$ ) to produce the desired product 53 (200 mg, 70\%, yellow oil). ${ }^{1} \mathrm{H}$ NMR (400 $\left.\mathrm{MHz}, \mathrm{CDCl}_{3}\right) \delta 7.28-7.23$ (m, 2H), 7.13-7.11 (m, 1H), 7.06-7.05 (m, 1H), $6.92(\mathrm{ddd}, J=7.7,1.6,0.9 \mathrm{~Hz}, 1 \mathrm{H}), 6.90-6.88(\mathrm{~m}, 1 \mathrm{H})$, $6.84(\mathrm{ddd}, J=8.3,2.5,0.9 \mathrm{~Hz}, 1 \mathrm{H}), 3.78(\mathrm{~s}, 3 \mathrm{H}), 3.60(\mathrm{t}, J=6.9 \mathrm{~Hz}, 2 \mathrm{H}), 3.49(\mathrm{t}, J=6.3 \mathrm{~Hz}, 2 \mathrm{H}), 2.56$ $(\mathrm{q}, J=7.6 \mathrm{~Hz}, 2 \mathrm{H}), 2.26(\mathrm{td}, J=13.2,6.6 \mathrm{~Hz}, 2 \mathrm{H}), 1.15(\mathrm{t}, J=7.6 \mathrm{~Hz}, 3 \mathrm{H}) ;{ }^{13} \mathrm{C} \mathrm{NMR}\left(100 \mathrm{MHz}, \mathrm{CDCl}_{3}\right)$ $\delta 160.4,145.4,137.2,135.0,133.2,132.4,130.4,128.2,126.3,123.8,116.7,115.1,113.9,55.5,52.4$, 30.8, 29.6, 28.4, 15.4; IR (neat) $v_{\max }=2965,2213,1588,1479,1403,1282,1245,1200,1036$, $857\left(\mathrm{~cm}^{-1}\right)$; HRMS (FAB): calcd. for $\mathrm{C}_{19} \mathrm{H}_{22} \mathrm{BrN}_{2} \mathrm{OS}[\mathrm{M}+\mathrm{H}]^{+}$405.0636, found 405.0635.

$N$-(3-Bromopropyl)- $N$-(3,4-difluorophenyl)-6-ethylbenzo[d] thiazol-2-amine (54). To a solution of compound 37 (300 mg, $1.03 \mathrm{mmol})$ in dry DMF (5.17 mL, $0.2 \mathrm{M})$ was added $\mathrm{NaH}(60 \%$ in mineral oil, $103 \mathrm{mg}, 2.58 \mathrm{mmol}$ ), and the mixture was stirred at $0{ }^{\circ} \mathrm{C}$ for $10 \mathrm{~min}$. To this reaction mixture was added 1,3-dibromo-propane $(1.05 \mathrm{~mL}, 10.3 \mathrm{mmol})$ and then the mixture was allowed to warm to room 
temperature slowly and stirred at $50{ }^{\circ} \mathrm{C}$ for $14 \mathrm{~h}$. The reaction mixture was diluted with $\mathrm{H}_{2} \mathrm{O}$ and extracted with EtOAc. The organic layer was dried over $\mathrm{MgSO}_{4}$, filtered and evaporated in vacuo. The crude product was separated by silica gel column chromatography (hexane/ether, $3: 1, \mathrm{R}_{\mathrm{f}}=0.2$ ) to produce the desired product $54\left(229 \mathrm{mg}, 54 \%\right.$, yellow oil). ${ }^{1} \mathrm{H}$ NMR $\left(400 \mathrm{MHz}, \mathrm{CDCl}_{3}\right) \delta 7.29(\mathrm{~d}, J=$ $8.1 \mathrm{~Hz}, 1 \mathrm{H}), 7.19-7.09(\mathrm{~m}, 4 \mathrm{H}), 7.00-6.99(\mathrm{~m}, 1 \mathrm{H}), 3.60(\mathrm{t}, J=6.9 \mathrm{~Hz}, 2 \mathrm{H}), 3.51(\mathrm{t}, J=6.3 \mathrm{~Hz}, 2 \mathrm{H})$, $2.56(\mathrm{q}, J=7.6 \mathrm{~Hz}, 2 \mathrm{H}), 2.27(\mathrm{td}, J=13.1,6.6 \mathrm{~Hz}, 2 \mathrm{H}), 1.16(\mathrm{t}, J=7.6 \mathrm{~Hz}, 3 \mathrm{H}) ;{ }^{13} \mathrm{C} \mathrm{NMR}(100 \mathrm{MHz}$, $\left.\mathrm{CDCl}_{3}\right) \delta 151.71(\mathrm{dd}, J=12.7,39.4 \mathrm{~Hz}, 1 \mathrm{C}), 149.22(\mathrm{dd}, J=12.8,37.7 \mathrm{~Hz}, 1 \mathrm{C}), 145.7,137.2,132.6$, $132.1,130.14(\mathrm{t}, J=4.9 \mathrm{~Hz}, 1 \mathrm{C}), 128.7,128.14(\mathrm{q}, J=3.2 \mathrm{~Hz}, 1 \mathrm{C}), 126.4,120.73(\mathrm{~d}, J=18.2 \mathrm{~Hz}, 1 \mathrm{C})$, $118.40(\mathrm{~d}, J=17.6 \mathrm{~Hz}, 1 \mathrm{C}), 114.8,52.4,30.6,29.5,28.3,15.3$; IR (neat) $v_{\max }=2968,2214,1600$, 1501, 1485, 1272, 1199, 1115, 902, $771\left(\mathrm{~cm}^{-1}\right)$; HRMS (ESI): calcd. for $\mathrm{C}_{18} \mathrm{H}_{18} \mathrm{BrF}_{2} \mathrm{~N}_{2} \mathrm{~S}[\mathrm{M}+\mathrm{H}]^{+}$ 411.0337, found 411.0326 .

$N$-(3-Bromopropyl)-6-ethyl- $N$-(4-(trifluoromethyl)phenyl)benzo[d]thiazol-2-amine (55). To a solution of compound $38(145 \mathrm{mg}, 0.45 \mathrm{mmol})$ in dry DMF $(2.25 \mathrm{~mL}, 0.2 \mathrm{M})$ was added $\mathrm{NaH}(60 \%$ in mineral oil, $45 \mathrm{mg}, 1.13 \mathrm{mmol}$ ), and the mixture was stirred at $0{ }^{\circ} \mathrm{C}$ for $10 \mathrm{~min}$. To this reaction mixture was added 1,3-dibromo-propane $(460 \mu \mathrm{L}, 4.50 \mathrm{mmol})$ and then the mixture was allowed to warm to room temperature slowly and stirred at $50{ }^{\circ} \mathrm{C}$ for $14 \mathrm{~h}$. The reaction mixture was diluted with $\mathrm{H}_{2} \mathrm{O}$ and extracted with EtOAc. The organic layer was dried over $\mathrm{MgSO}_{4}$, filtered and evaporated in vacuo. The crude product was separated by silica gel column chromatography (hexane/ether, $3: 1, \mathrm{R}_{\mathrm{f}}=0.2$ ) to produce the desired product $55\left(120 \mathrm{mg}, 60 \%\right.$, yellow oil). ${ }^{1} \mathrm{H}$ NMR $\left(400 \mathrm{MHz}, \mathrm{CDCl}_{3}\right) \delta 7.54(\mathrm{~d}, J=$ $8.4 \mathrm{~Hz}, 2 \mathrm{H}), 7.36-7.29(\mathrm{~m}, 3 \mathrm{H}), 7.26-7.24(\mathrm{~m}, 2 \mathrm{H}), 3.59(\mathrm{t}, J=6.9 \mathrm{~Hz}, 2 \mathrm{H}), 3.46(\mathrm{t}, J=6.2 \mathrm{~Hz}, 2 \mathrm{H})$, $2.62(\mathrm{q}, J=7.6 \mathrm{~Hz}, 2 \mathrm{H}), 2.23(\mathrm{td}, J=13.1,7.2 \mathrm{~Hz}, 2 \mathrm{H}), 1.20(\mathrm{t}, J=7.6 \mathrm{~Hz}, 3 \mathrm{H}) ;{ }^{13} \mathrm{C} \mathrm{NMR}(100 \mathrm{MHz}$, $\left.\mathrm{CDCl}_{3}\right) \delta 145.8,140.7,139.1134 .8,130.1,129.9,129.2(2 \mathrm{C}), 129.07$ (q, $\left.J=32.8 \mathrm{~Hz}, 1 \mathrm{C}\right), 126.4,126.3$ $(\mathrm{q}, J=3.7 \mathrm{~Hz}, 2 \mathrm{C}), 123.92(\mathrm{q}, J=220.8 \mathrm{~Hz}, 1 \mathrm{C}), 114.8,52.5,30.6,29.5,28.4,15.4$; IR (neat) $v_{\max }=$ 2969, 2216, 1605, 1485, 1324, 1164, 1122, 1063, 1014, $828\left(\mathrm{~cm}^{-1}\right)$; HRMS (ESI): calcd. for $\mathrm{C}_{19} \mathrm{H}_{19} \mathrm{BrF}_{3} \mathrm{~N}_{2} \mathrm{~S}[\mathrm{M}+\mathrm{H}]^{+}$443.0399, found 443.0405. 


\section{B. Synthetic Procedures for the Preparation of 12-14}

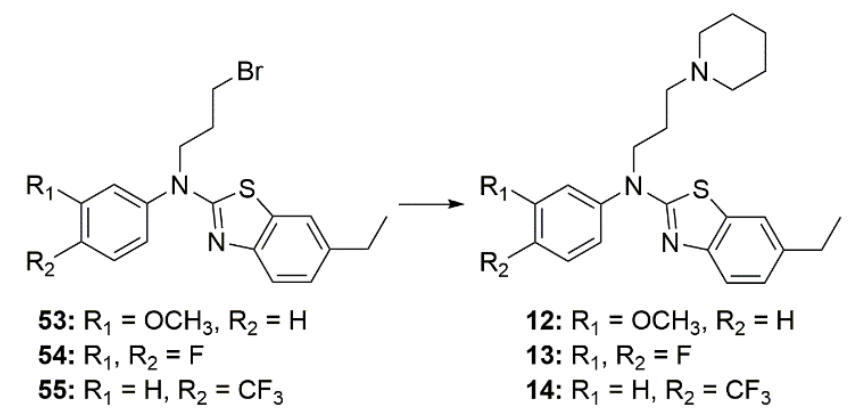

6-Ethyl- $N$-(3-methoxyphenyl)- $N$-(3-(piperidin-1-yl)propyl)benzo[d] thiazol-2-amine (12). То a solution of $53(200 \mathrm{mg}, 0.49 \mathrm{mmol})$ in $\mathrm{DMF} / \mathrm{MeCN}(1: 1,5 \mathrm{~mL}, 0.1 \mathrm{M})$ was added $\mathrm{K}_{2} \mathrm{CO}_{3}(273 \mathrm{mg}$, $1.97 \mathrm{mmol}$ ) and piperidine ( $484 \mu \mathrm{L}, 4.90 \mathrm{mmol})$. The reaction mixture was stirred at $50{ }^{\circ} \mathrm{C}$ for $14 \mathrm{~h}$ and then diluted with $\mathrm{H}_{2} \mathrm{O}$ and extracted with EtOAc. The organic layer was dried over $\mathrm{MgSO}_{4}$, filtered and evaporated in vacuo. The crude product was separated by silica gel column chromatography $\left(\mathrm{CH}_{2} \mathrm{Cl}_{2} / \mathrm{MeOH}, 20: 1, \mathrm{R}_{\mathrm{f}}=0.2\right)$ to produce the desired product $12(84.0 \mathrm{mg}, 42 \%$, colorless oil $) .{ }^{1} \mathrm{H}$ NMR $\left(400 \mathrm{MHz}, \mathrm{CDCl}_{3}\right) \delta 7.28(\mathrm{~d}, J=8.0 \mathrm{~Hz}, 1 \mathrm{H}), 7.26-7.22(\mathrm{~m}, 1 \mathrm{H}), 7.09(\mathrm{dd}, J=8.0,2.0 \mathrm{~Hz}, 1 \mathrm{H})$, 7.04-7.03 (m, 1H), 6.92-6.89 (m, 1H), 6.89-6.88 (m, 1H), 6.83 (ddd, $J=8.2,2.5,0.7 \mathrm{~Hz}, 1 \mathrm{H}), 3.77$ (s, $3 \mathrm{H}), 3.46(\mathrm{t}, J=7.3 \mathrm{~Hz}, 2 \mathrm{H}), 2.54(\mathrm{q}, J=7.6 \mathrm{~Hz}, 2 \mathrm{H}), 2.38-2.32(\mathrm{~m}, 6 \mathrm{H}), 1.88(\mathrm{td}, J=14.6,7.3 \mathrm{~Hz}$, $2 \mathrm{H}), 1.57-1.51(\mathrm{~m}, 4 \mathrm{H}), 1.43-1.39(\mathrm{~m}, 2 \mathrm{H}) 1.14(\mathrm{t}, J=7.6 \mathrm{~Hz}, 3 \mathrm{H}) ;{ }^{13} \mathrm{C}$ NMR $\left(100 \mathrm{MHz}, \mathrm{CDCl}_{3}\right) \delta$ $160.3,145.1,137.5,135.2,133.0,132.2,130.3,128.1,126.4,123.8,116.6,115.6,113.8,55.7,55.5$, 54.5 (2C), 52.5, 28.4, 25.9 (2C), 25.2, 24.4, 15.4; IR (neat) $v_{\max }=2933,2772,2213,1589,1480,1426$, 1230, 1038, 857, 774, $687\left(\mathrm{~cm}^{-1}\right)$; HRMS (FAB): calcd. for $\mathrm{C}_{24} \mathrm{H}_{32} \mathrm{~N}_{3} \mathrm{OS}[\mathrm{M}+\mathrm{H}]^{+} 410.2266$, found 410.2260 .

$N$-(3,4-Difluorophenyl)-6-ethyl- $N$-(3-(piperidin-1-yl)propyl)benzo[d] thiazol-2-amine (13). То а solution of 54 (55 mg, $0.13 \mathrm{mmol})$ in $\mathrm{DMF} / \mathrm{MeCN}(1: 1,1.34 \mathrm{~mL}, 0.1 \mathrm{M})$ was added $\mathrm{K}_{2} \mathrm{CO}_{3}(72 \mathrm{mg}$, $0.52 \mathrm{mmol})$ and piperidine $(128 \mu \mathrm{L}, 1.30 \mathrm{mmol})$. The reaction mixture was stirred at $50{ }^{\circ} \mathrm{C}$ for $16 \mathrm{~h}$ and then diluted with $\mathrm{H}_{2} \mathrm{O}$ and extracted with EtOAc. The organic layer was dried over $\mathrm{MgSO}_{4}$, filtered and evaporated in vacuo. The crude product was separated by silica gel column chromatography 
$\left(\mathrm{CH}_{2} \mathrm{Cl}_{2} / \mathrm{MeOH}, 20: 1, \mathrm{R}_{\mathrm{f}}=0.25\right)$ to produce the desired product $13\left(30.0 \mathrm{mg}, 56 \%\right.$, white solid). ${ }^{1} \mathrm{H}$ NMR (400 MHz, $\left.\mathrm{CDCl}_{3}\right) \delta 7.29(\mathrm{~d}, J=8.1 \mathrm{~Hz}, 1 \mathrm{H}), 7.18-7.07(\mathrm{~m}, 4 \mathrm{H}), 7.00-6.99(\mathrm{~m}, 1 \mathrm{H}), 3.46(\mathrm{t}, J$ $=7.6 \mathrm{~Hz}, 2 \mathrm{H}), 2.56(\mathrm{q}, J=7.6 \mathrm{~Hz}, 2 \mathrm{H}), 2.38-2.32(\mathrm{~m}, 6 \mathrm{H}), 1.88(\mathrm{td}, J=14.5,7.2 \mathrm{~Hz}, 2 \mathrm{H}), 1.56-1.50$ $(\mathrm{m}, 4 \mathrm{H}), 1.43-1.39(\mathrm{~m}, 2 \mathrm{H}), 1.15(\mathrm{t}, J=7.6 \mathrm{~Hz}, 3 \mathrm{H}) ;{ }^{13} \mathrm{C}$ NMR $\left(100 \mathrm{MHz}, \mathrm{CDCl}_{3}\right) \delta 151.70(\mathrm{dd}, J=$ $12.9,45.7 \mathrm{~Hz}, 1 \mathrm{C}), 149.20(\mathrm{dd}, J=12.7,44.1 \mathrm{~Hz}, 1 \mathrm{C}), 145.3,137.7,132.4,132.1,130.52(\mathrm{t}, J=5.0$ $\mathrm{Hz}, 1 \mathrm{C}), 128.6,128.04$ (q, $J=3.3 \mathrm{~Hz}, 1 \mathrm{C}), 126.5,120.65(\mathrm{~d}, J=18.2 \mathrm{~Hz}, 1 \mathrm{C}), 118.34(\mathrm{~d}, J=17.7 \mathrm{~Hz}$ 1C), 115.4, 55.7, 54.6 (2C), 52.6, 28.4, 26.1 (2C), 25.3, 24.5, 15.4; IR (neat) $v_{\max }=2934,2214,1600$, 1501, 1486, 1403, 1272, 1200, 1116, 903, $771\left(\mathrm{~cm}^{-1}\right)$; HRMS (FAB): calcd. for $\mathrm{C}_{23} \mathrm{H}_{28} \mathrm{~F}_{2} \mathrm{~N}_{3} \mathrm{~S}[\mathrm{M}+\mathrm{H}]^{+}$ 416.1972, found 416.1964 .

\section{6-Ethyl- $N$-(3-(piperidin-1-yl)propyl)- $N$-(4-(trifluoromethyl)phenyl)benzo[d] thiazol-2-amine (14).}

To a solution of $\mathbf{5 5}(80 \mathrm{mg}, 0.18 \mathrm{mmol})$ in $\mathrm{DMF} / \mathrm{MeCN}(1: 1,1.81 \mathrm{~mL}, 0.1 \mathrm{M})$ was added $\mathrm{K}_{2} \mathrm{CO}_{3}(100$ $\mathrm{mg}, 0.72 \mathrm{mmol}$ ) and piperidine ( $54 \mu \mathrm{L}, 0.54 \mathrm{mmol})$. The reaction mixture was stirred at $50{ }^{\circ} \mathrm{C}$ for $14 \mathrm{~h}$ and then diluted with $\mathrm{H}_{2} \mathrm{O}$ and extracted with EtOAc. The organic layer was dried over $\mathrm{MgSO}_{4}$, filtered and evaporated in vacuo. The crude product was separated by silica gel column chromatography $\left(\mathrm{CH}_{2} \mathrm{Cl}_{2} / \mathrm{MeOH}, 20: 1, \mathrm{R}_{\mathrm{f}}=0.2\right)$ to produce the desired product 14 (45.0 mg, 56\%, yellow oil). ${ }^{1} \mathrm{H}$ NMR $\left(400 \mathrm{MHz}, \mathrm{CDCl}_{3}\right) \delta 7.49(\mathrm{~d}, J=8.3 \mathrm{~Hz}, 2 \mathrm{H}), 7.34-7.31(\mathrm{~m}, 1 \mathrm{H}), 7.26-7.23(\mathrm{~m}, 2 \mathrm{H}), 7.20-7.19(\mathrm{~m}$, 2H), $3.43(\mathrm{t}, J=7.3 \mathrm{~Hz}, 2 \mathrm{H}), 2.57(\mathrm{q}, J=7.6 \mathrm{~Hz}, 2 \mathrm{H}), 2.31-2.28(\mathrm{~m}, 6 \mathrm{H}), 1.81(\mathrm{td}, J=14.4,7.2 \mathrm{~Hz}$, 2H), $1.52-1.46(\mathrm{~m}, 4 \mathrm{H}), 1.38-1.37(\mathrm{~m}, 2 \mathrm{H}), 1.16(\mathrm{t}, J=7.6 \mathrm{~Hz}, 3 \mathrm{H}) ;{ }^{13} \mathrm{C} \mathrm{NMR}\left(100 \mathrm{MHz}, \mathrm{CDCl}_{3}\right) \delta$ $145.4,141.0,139.5,134.9,129.8,129.6,129.0(2 \mathrm{C}), 128.83(\mathrm{q}, J=32.4 \mathrm{~Hz}, 1 \mathrm{C}), 126.5,126.19$ (q, $J=$ 3.7 Hz, 2C), 124.1 (q, $J=269.6 \mathrm{~Hz}, 1 \mathrm{C}), 115.3,55.6,54.6$ (2C), 52.7, 28.3, 26.0 (2C), 25.2, 24.4, 15.4; IR (neat) $v_{\max }=2935,2214,1605,1486,1323,1163,1122,1089,1062,1013,827\left(\mathrm{~cm}^{-1}\right)$; HRMS (FAB): calcd. for $\mathrm{C}_{24} \mathrm{H}_{29} \mathrm{~F}_{3} \mathrm{~N}_{3} \mathrm{~S}[\mathrm{M}+\mathrm{H}]^{+} 448.2034$, found 448.2024 . 


\section{NMR Spectra}

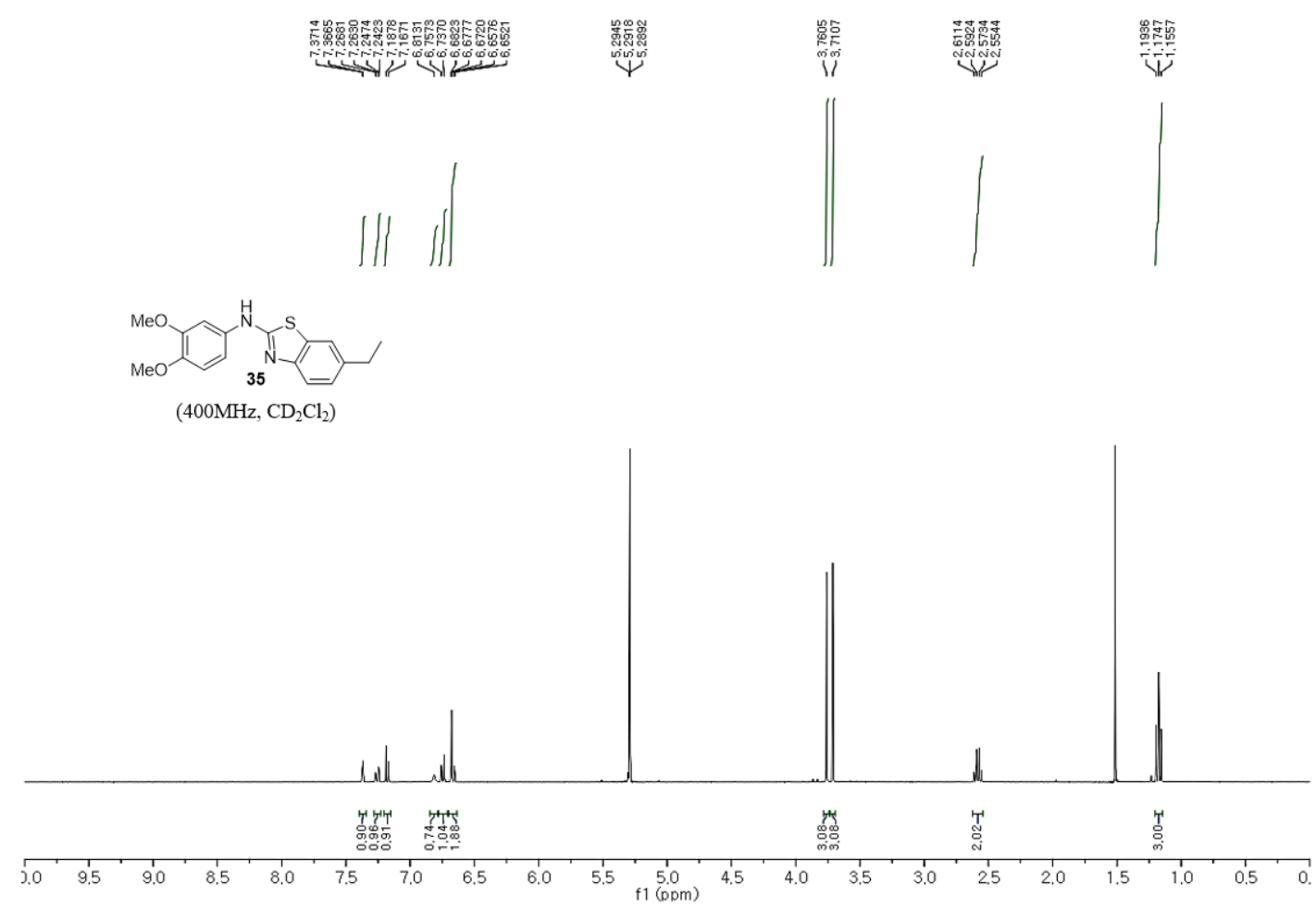

${ }^{1} \mathrm{H}$ NMR spectrum (400 MHz) of $\mathbf{3 5}$ in $\mathrm{CD}_{2} \mathrm{Cl}_{2}$

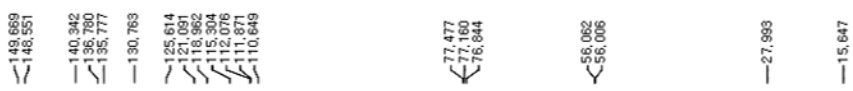
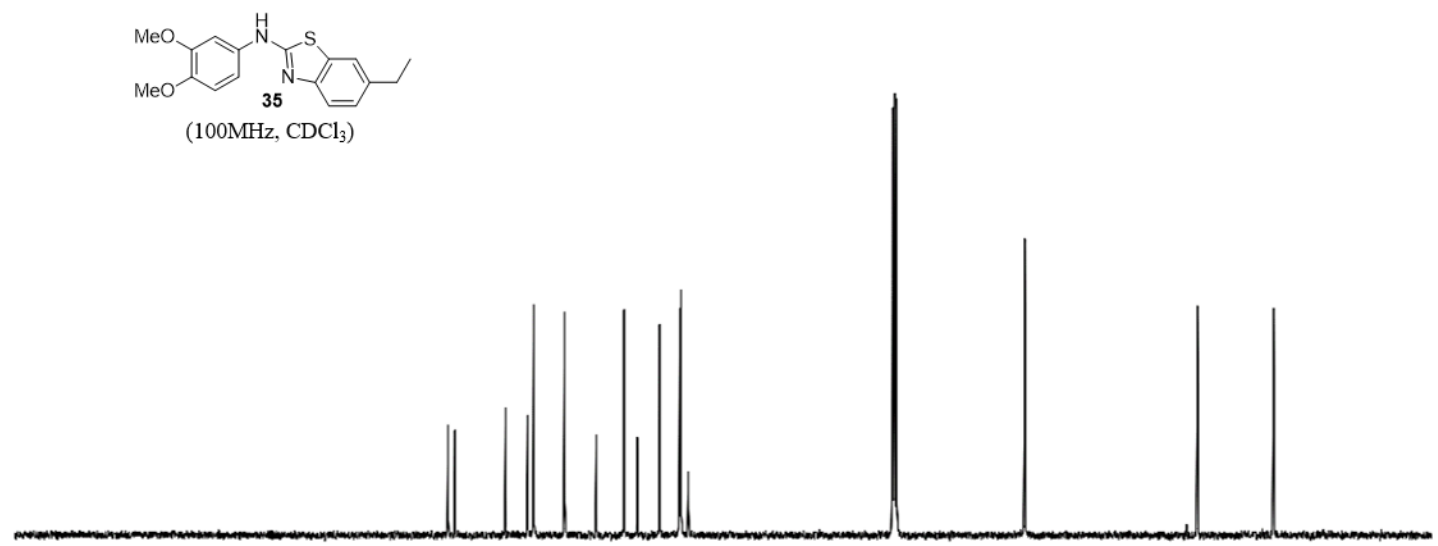

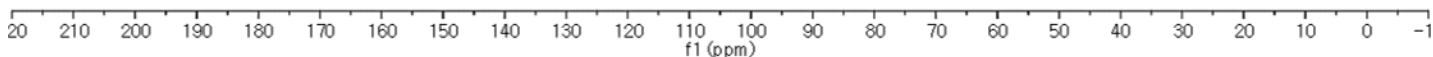

${ }^{13} \mathrm{C}$ NMR spectrum $(100 \mathrm{MHz})$ of 35 in $\mathrm{CDCl}_{3}$ 


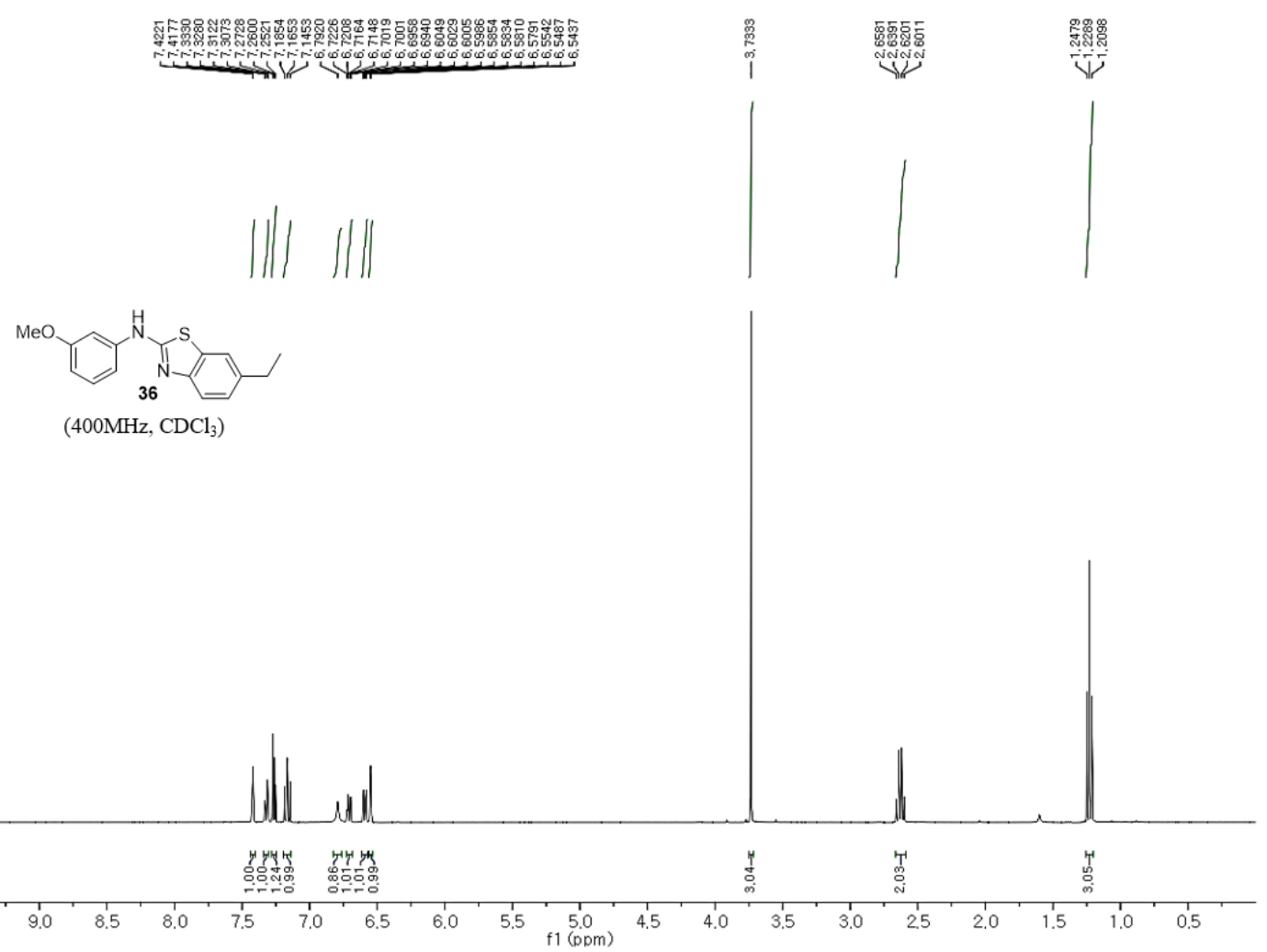

${ }^{1} \mathrm{H}$ NMR spectrum (400 MHz) of $\mathbf{3 6}$ in $\mathrm{CDCl}_{3}$

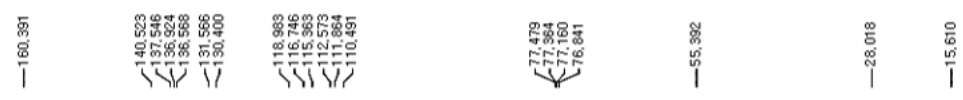

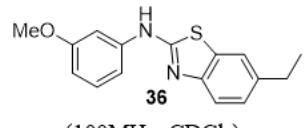

$\left(100 \mathrm{MHz}, \mathrm{CDCl}_{3}\right.$ )

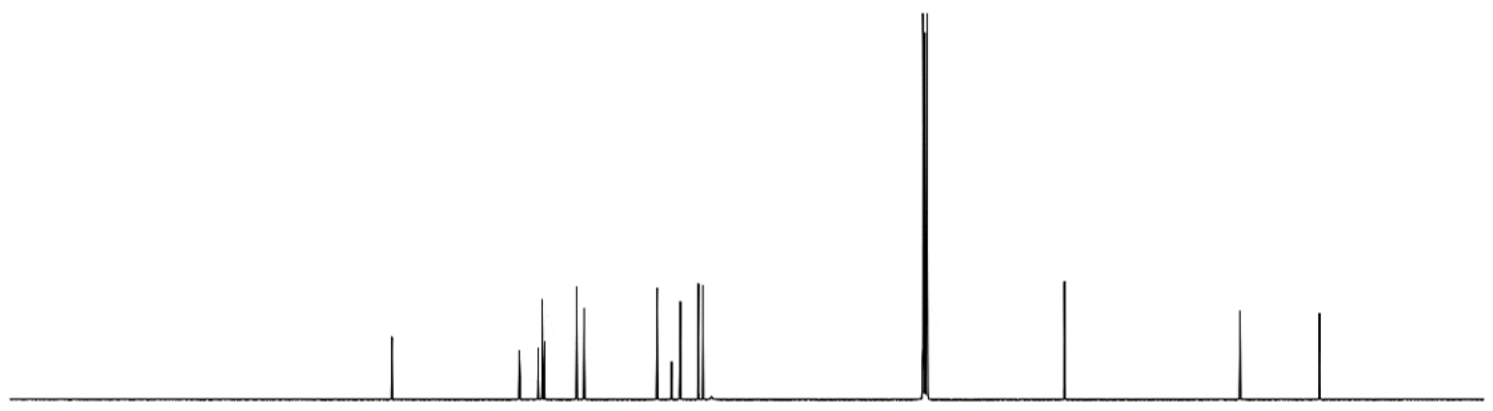

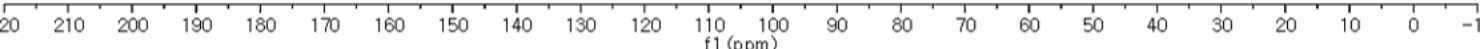

${ }^{13} \mathrm{C}$ NMR spectrum $(100 \mathrm{MHz})$ of $\mathbf{3 6}$ in $\mathrm{CDCl}_{3}$ 

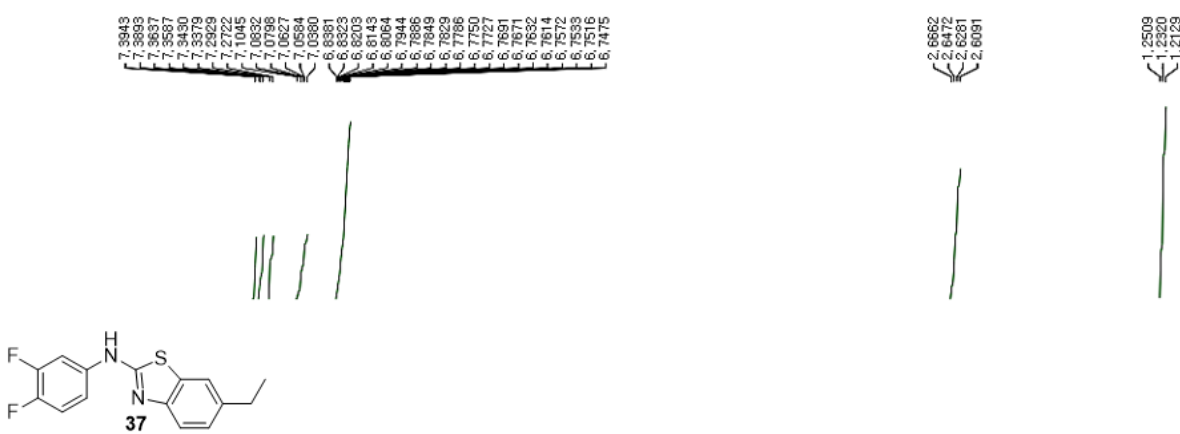

$\left(400 \mathrm{MHz}, \mathrm{CDCl}_{3}\right)$

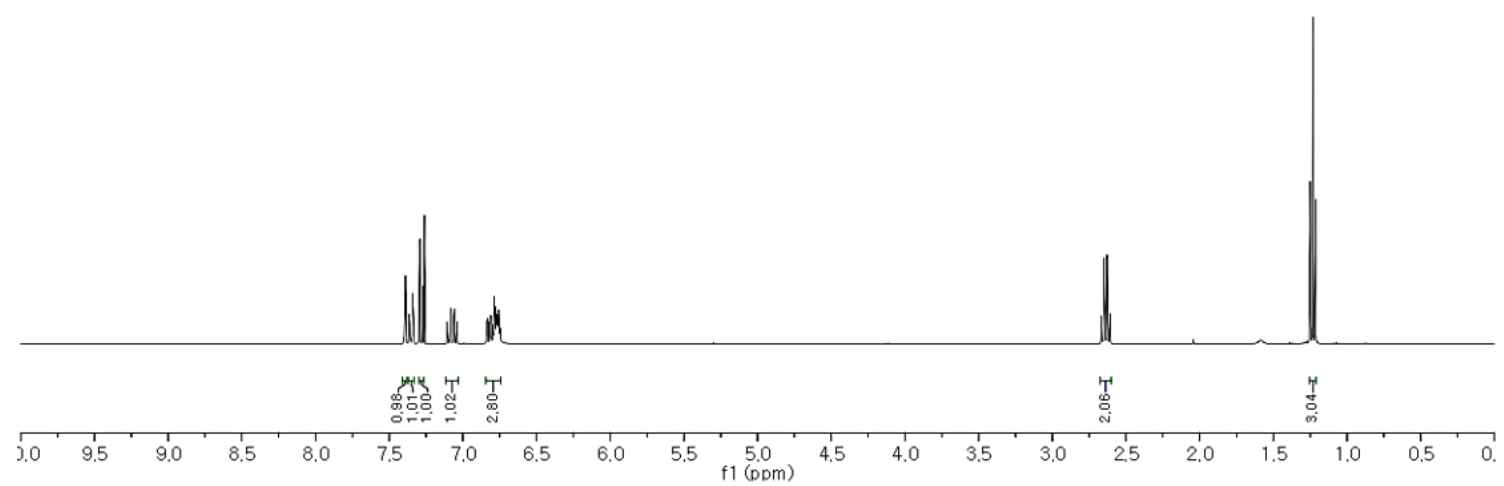

${ }^{1} \mathrm{H}$ NMR spectrum (400 MHz) of 37 in $\mathrm{CDCl}_{3}$

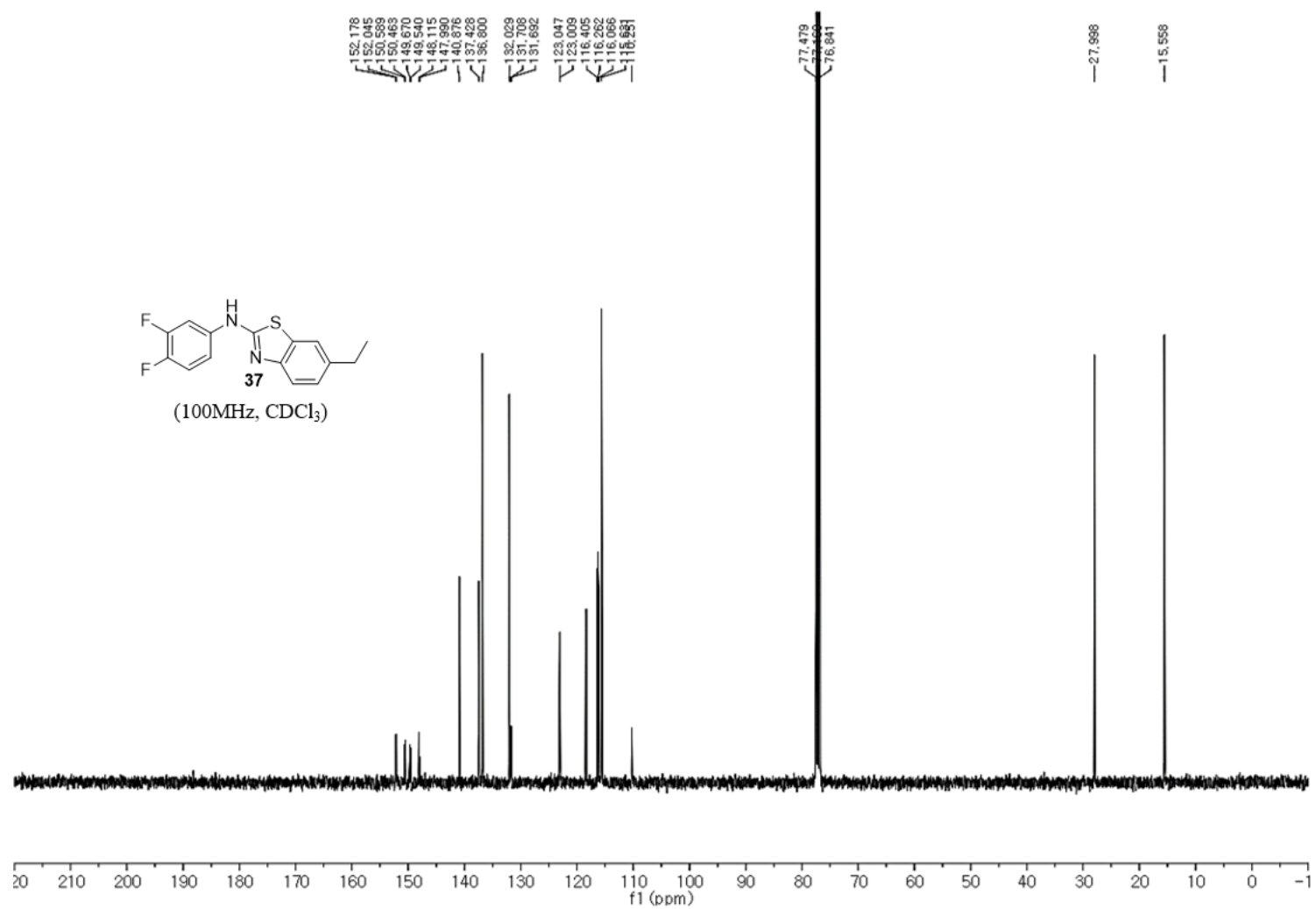

${ }^{13} \mathrm{C}$ NMR spectrum $(100 \mathrm{MHz})$ of $\mathbf{3 7}$ in $\mathrm{CDCl}_{3}$ 

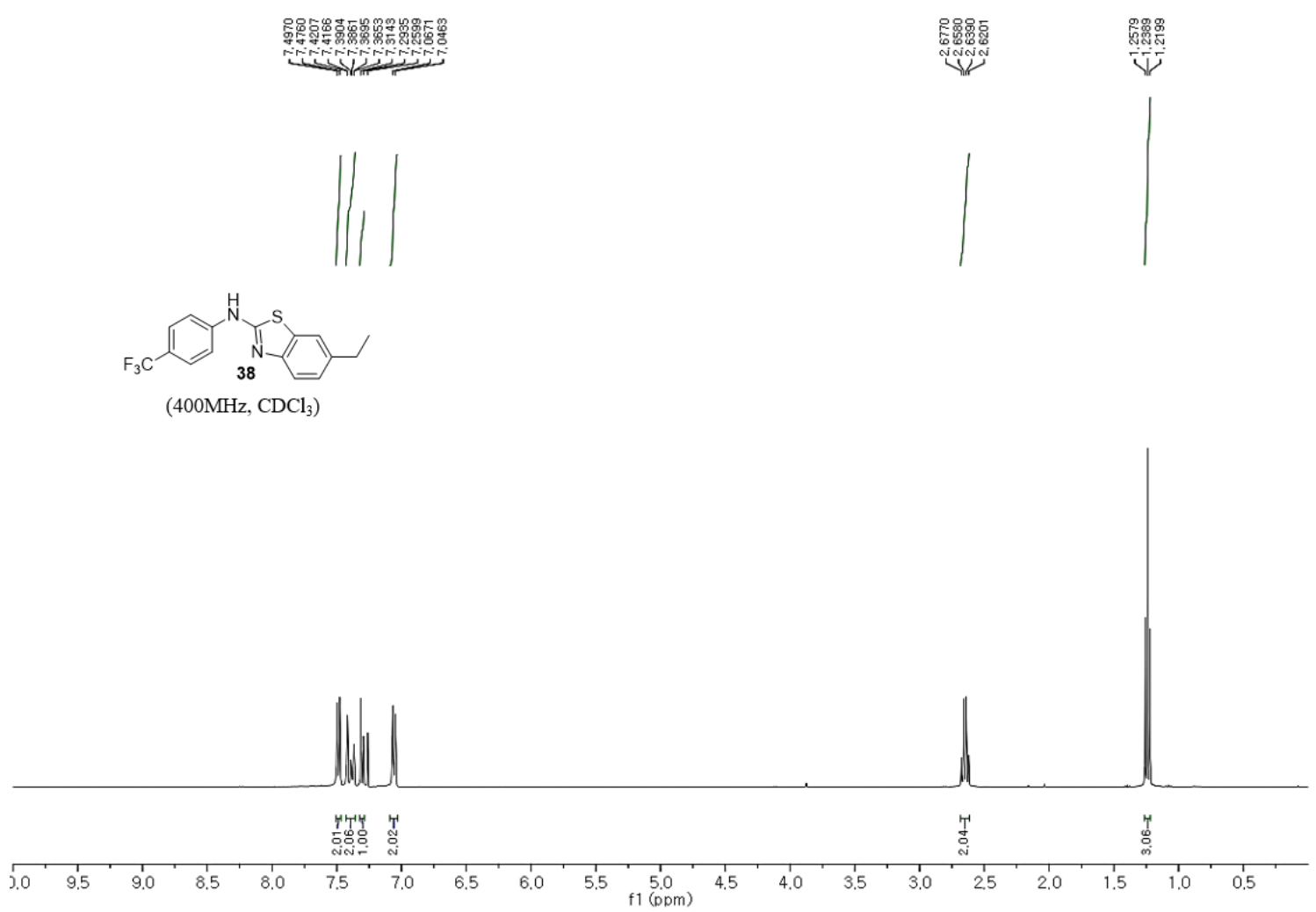

${ }^{1} \mathrm{H}$ NMR spectrum $(400 \mathrm{MHz})$ of 38 in $\mathrm{CDCl}_{3}$

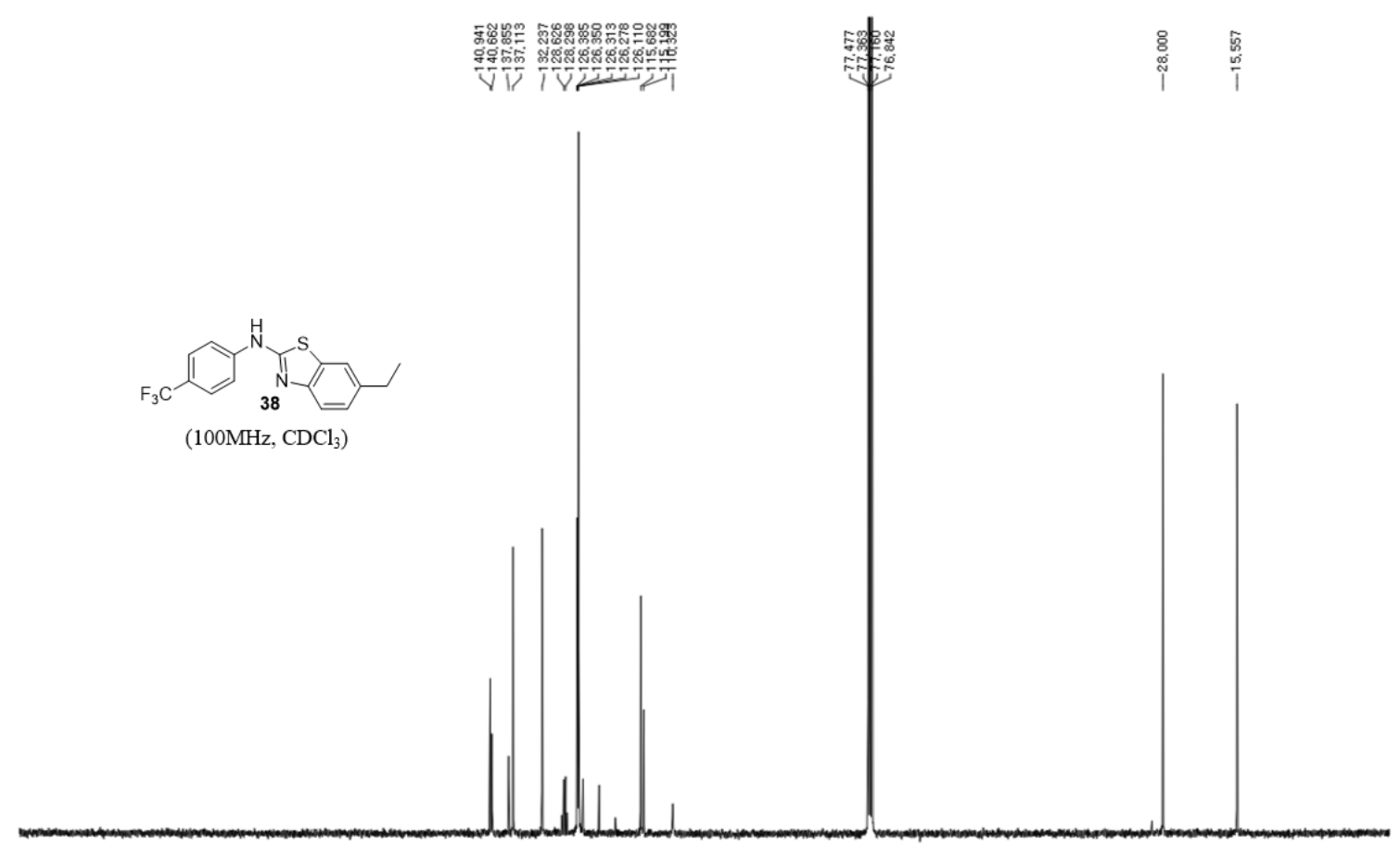

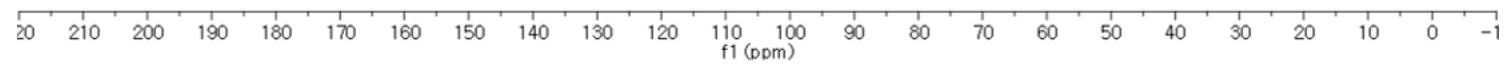

${ }^{13} \mathrm{C}$ NMR spectrum $(100 \mathrm{MHz})$ of $\mathbf{3 8}$ in $\mathrm{CDCl}_{3}$ 

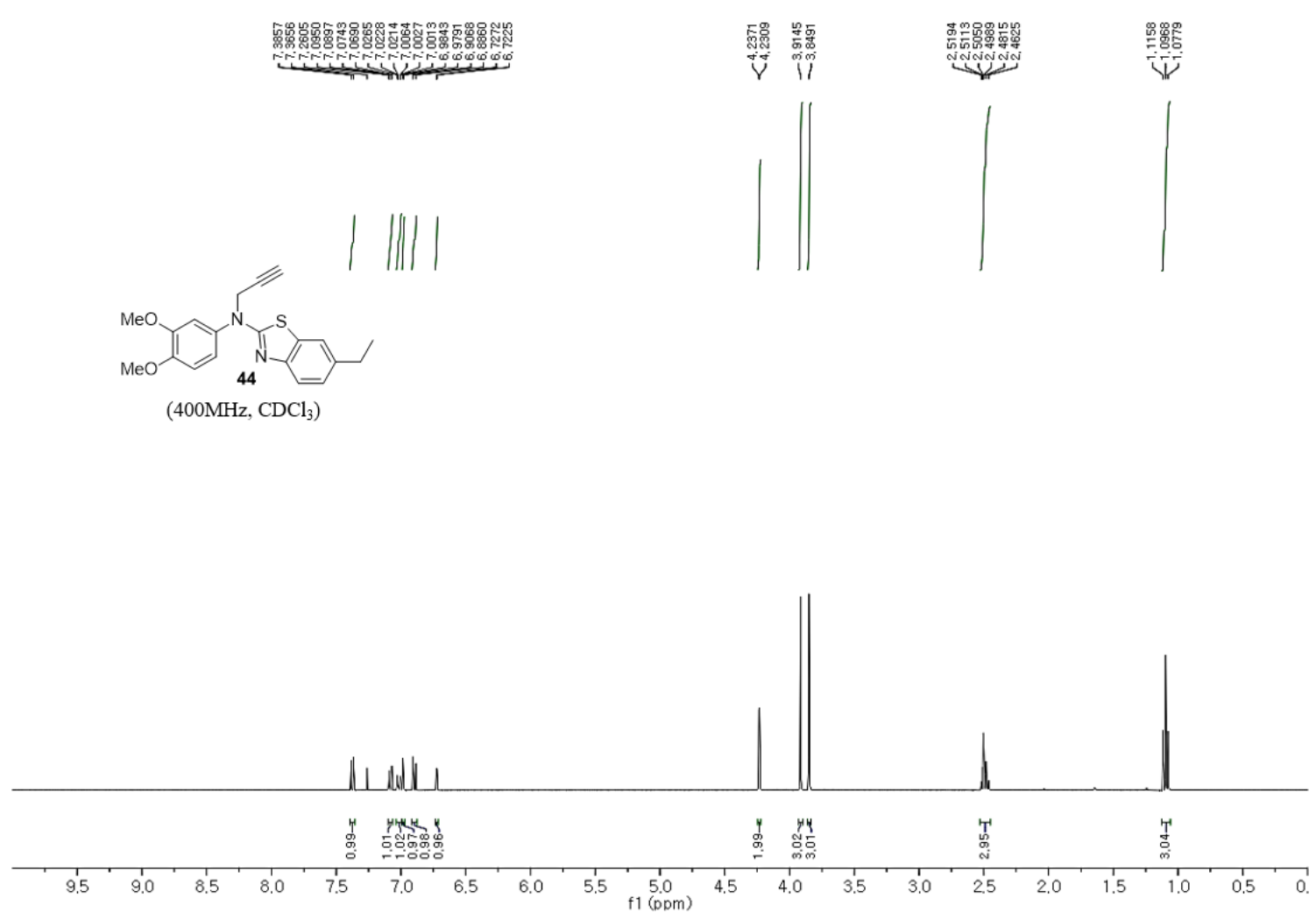

${ }^{1} \mathrm{H}$ NMR spectrum $(400 \mathrm{MHz})$ of 44 in $\mathrm{CDCl}_{3}$

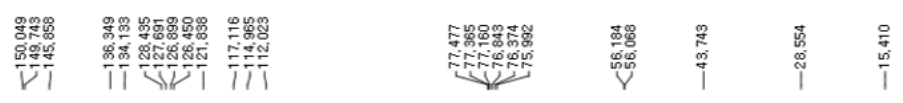

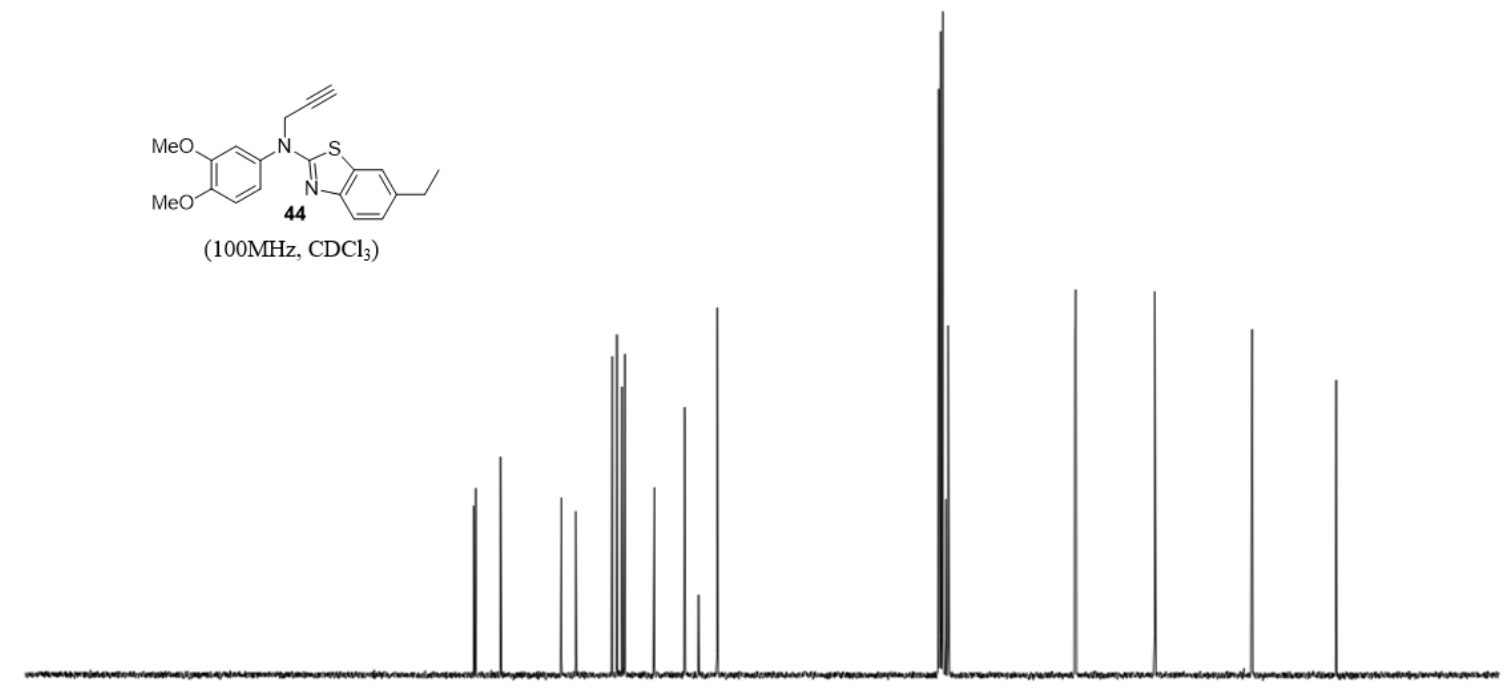

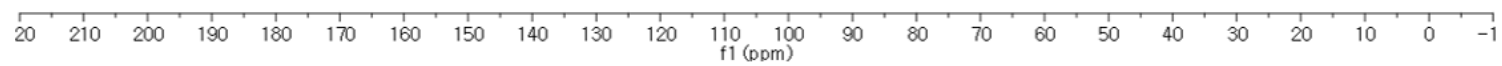

${ }^{13} \mathrm{C}$ NMR spectrum $(100 \mathrm{MHz})$ of $\mathbf{4 4}$ in $\mathrm{CDCl}_{3}$ 


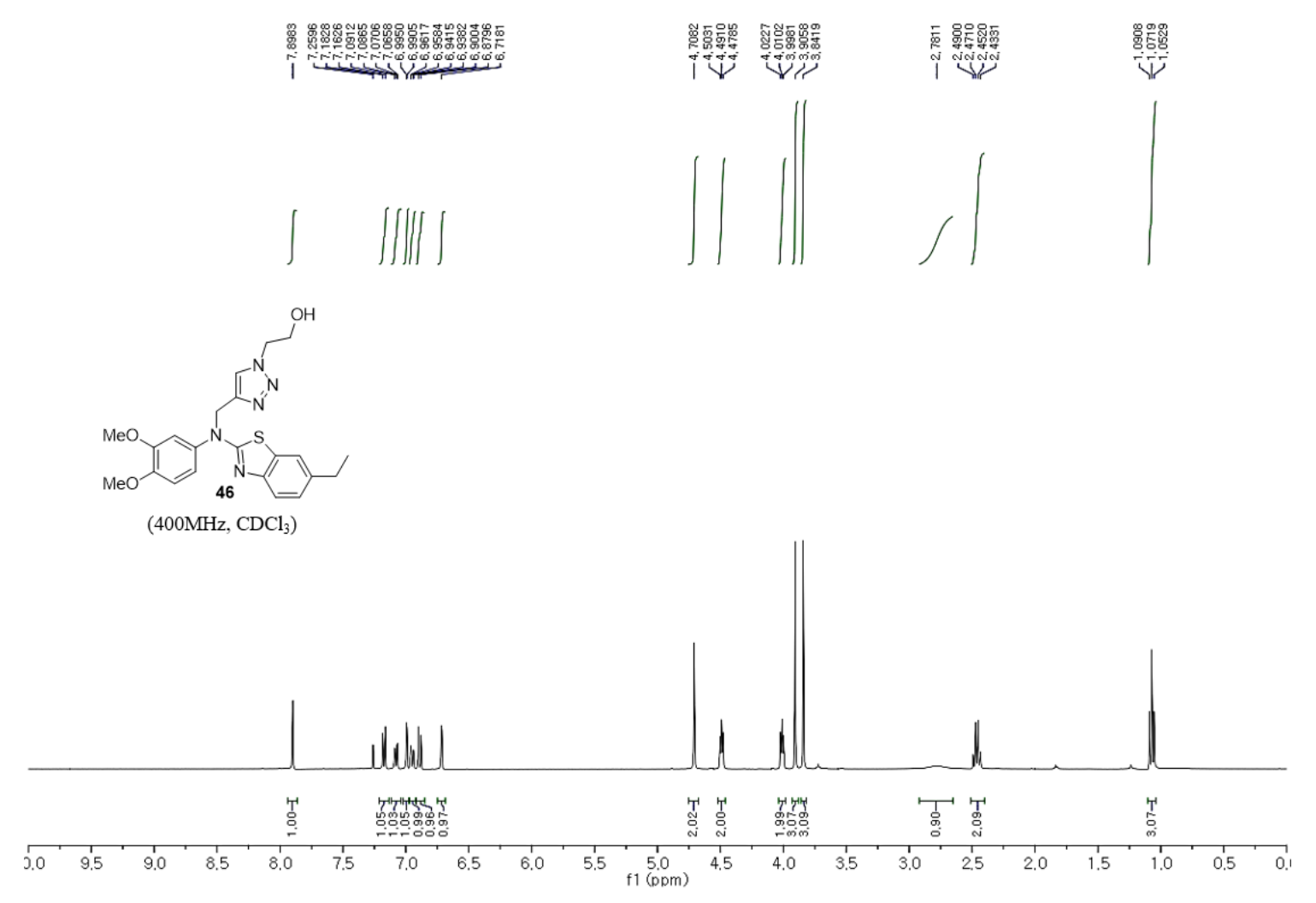

${ }^{1} \mathrm{H}$ NMR spectrum (400 MHz) of 46 in $\mathrm{CDCl}_{3}$

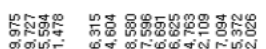

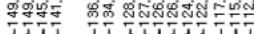

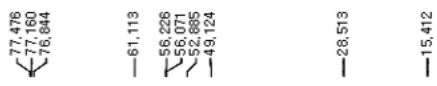
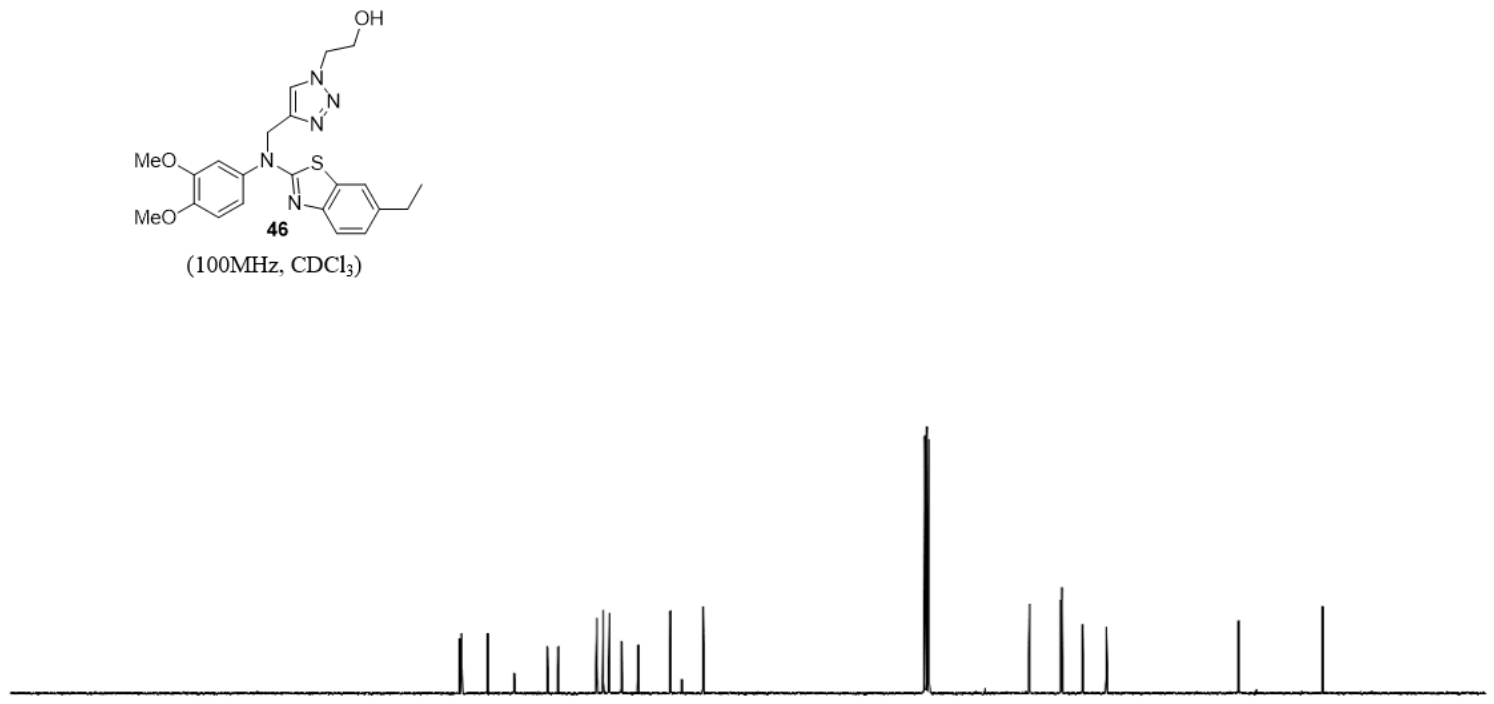

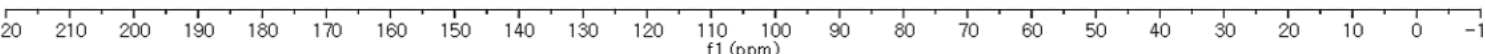

${ }^{13} \mathrm{C}$ NMR spectrum $(100 \mathrm{MHz})$ of 46 in $\mathrm{CDCl}_{3}$ 


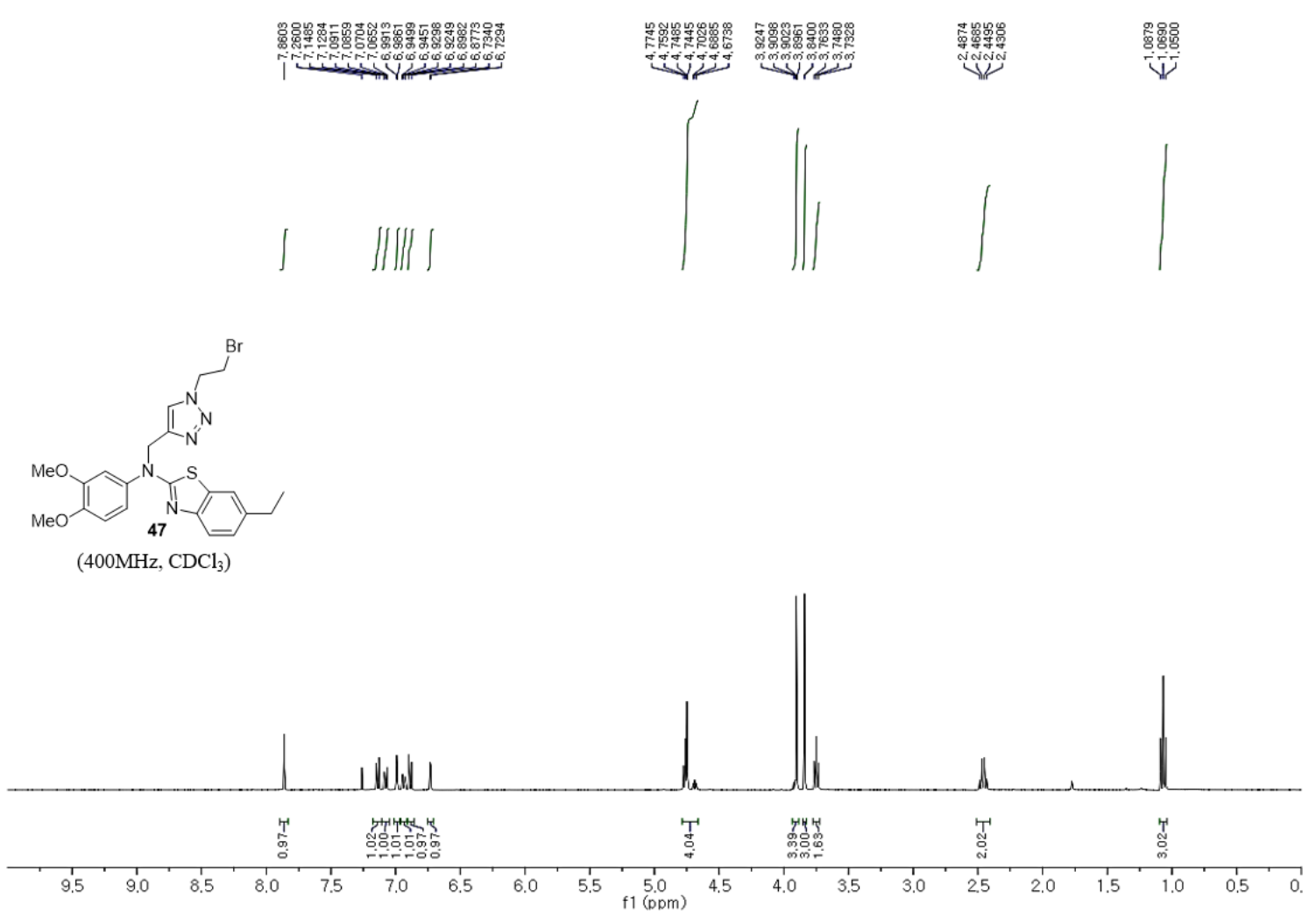

${ }^{1} \mathrm{H}$ NMR spectrum (400 MHz) of 47 in $\mathrm{CDCl}_{3}$

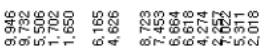

察守守守

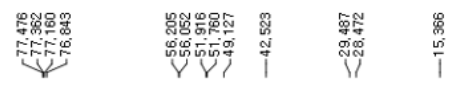
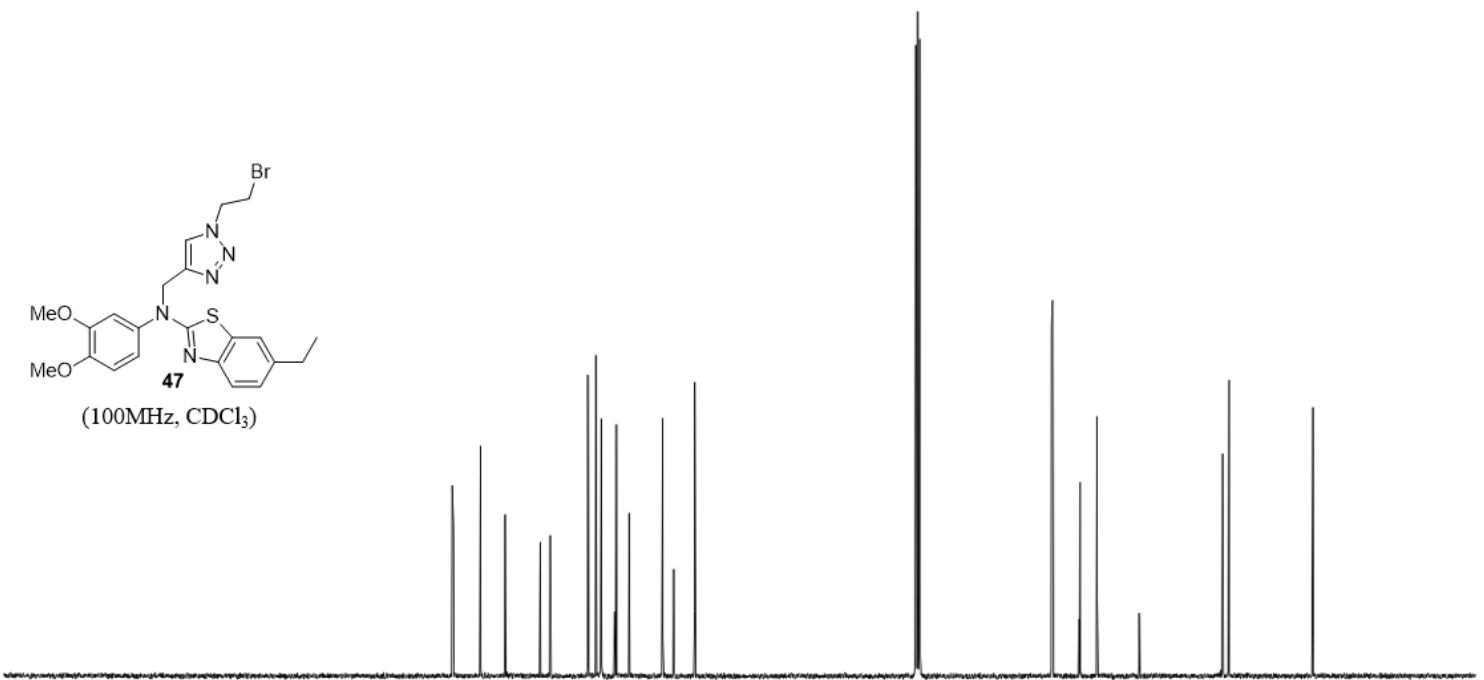

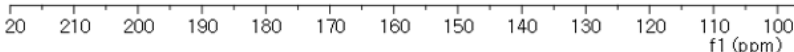

${ }^{13} \mathrm{C}$ NMR spectrum $(100 \mathrm{MHz})$ of 47 in $\mathrm{CDCl}_{3}$ 


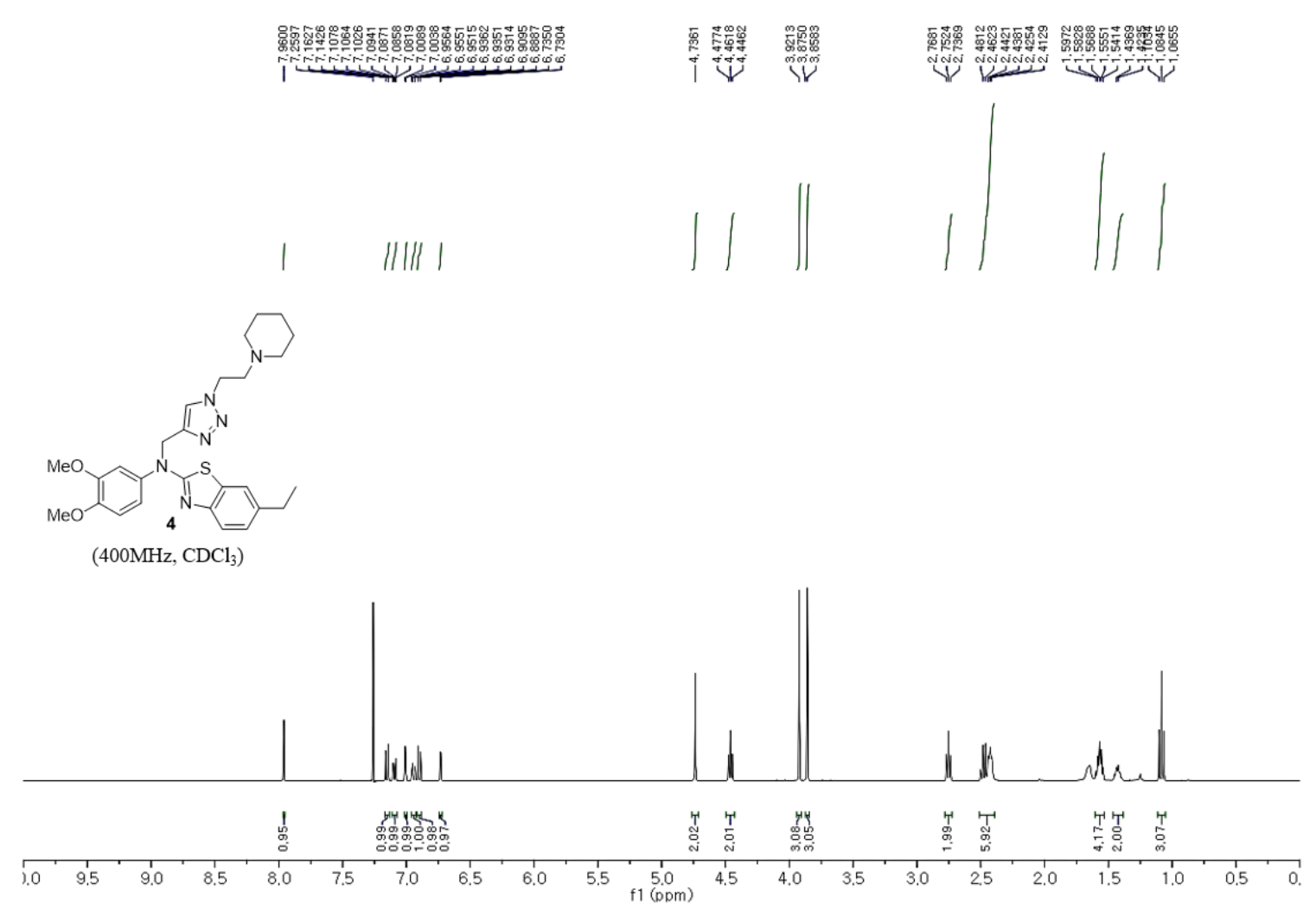

${ }^{1} \mathrm{H}$ NMR spectrum $(400 \mathrm{MHz})$ of 4 in $\mathrm{CDCl}_{3}$
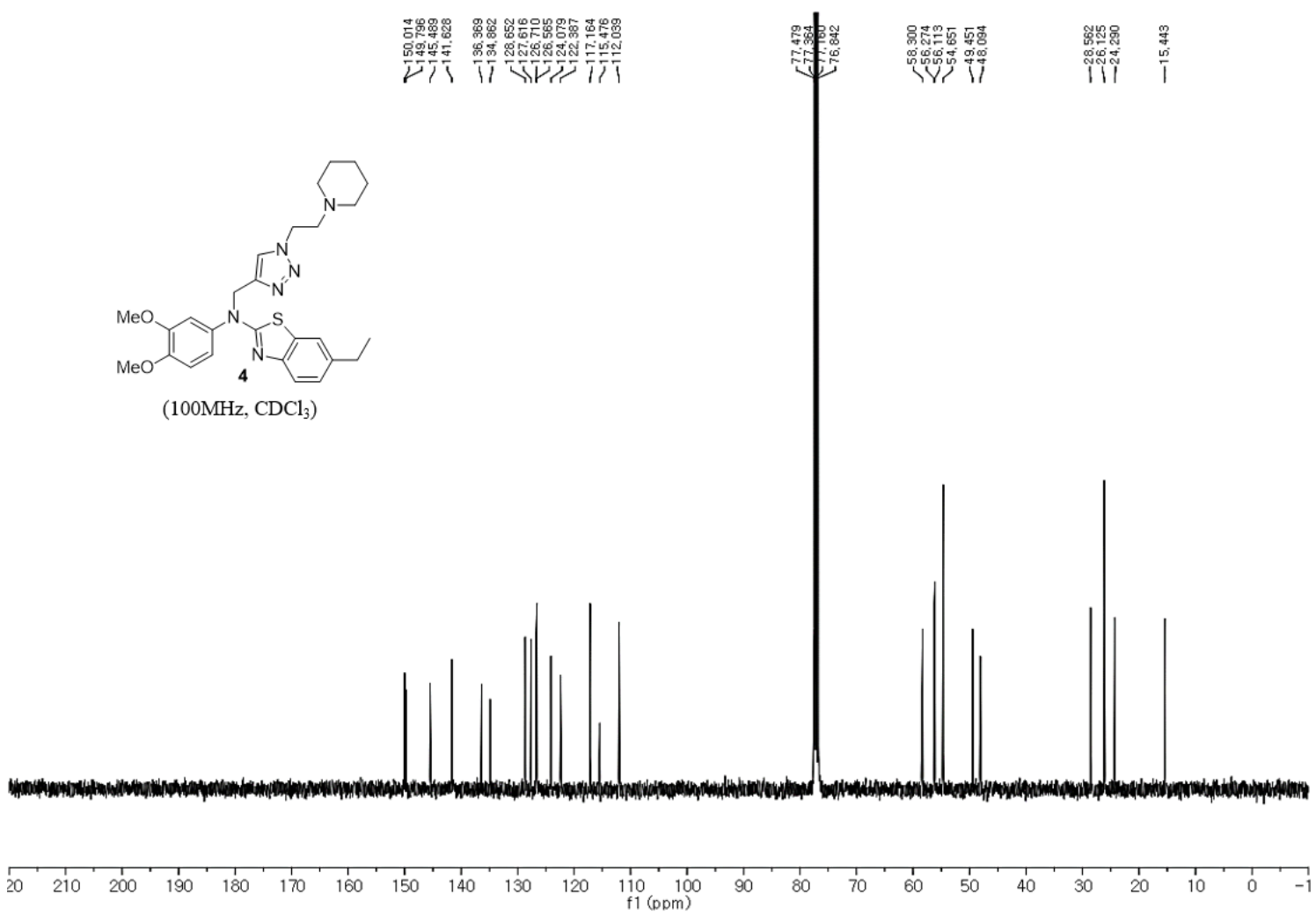

${ }^{13} \mathrm{C}$ NMR spectrum $(100 \mathrm{MHz})$ of 4 in $\mathrm{CDCl}_{3}$ 


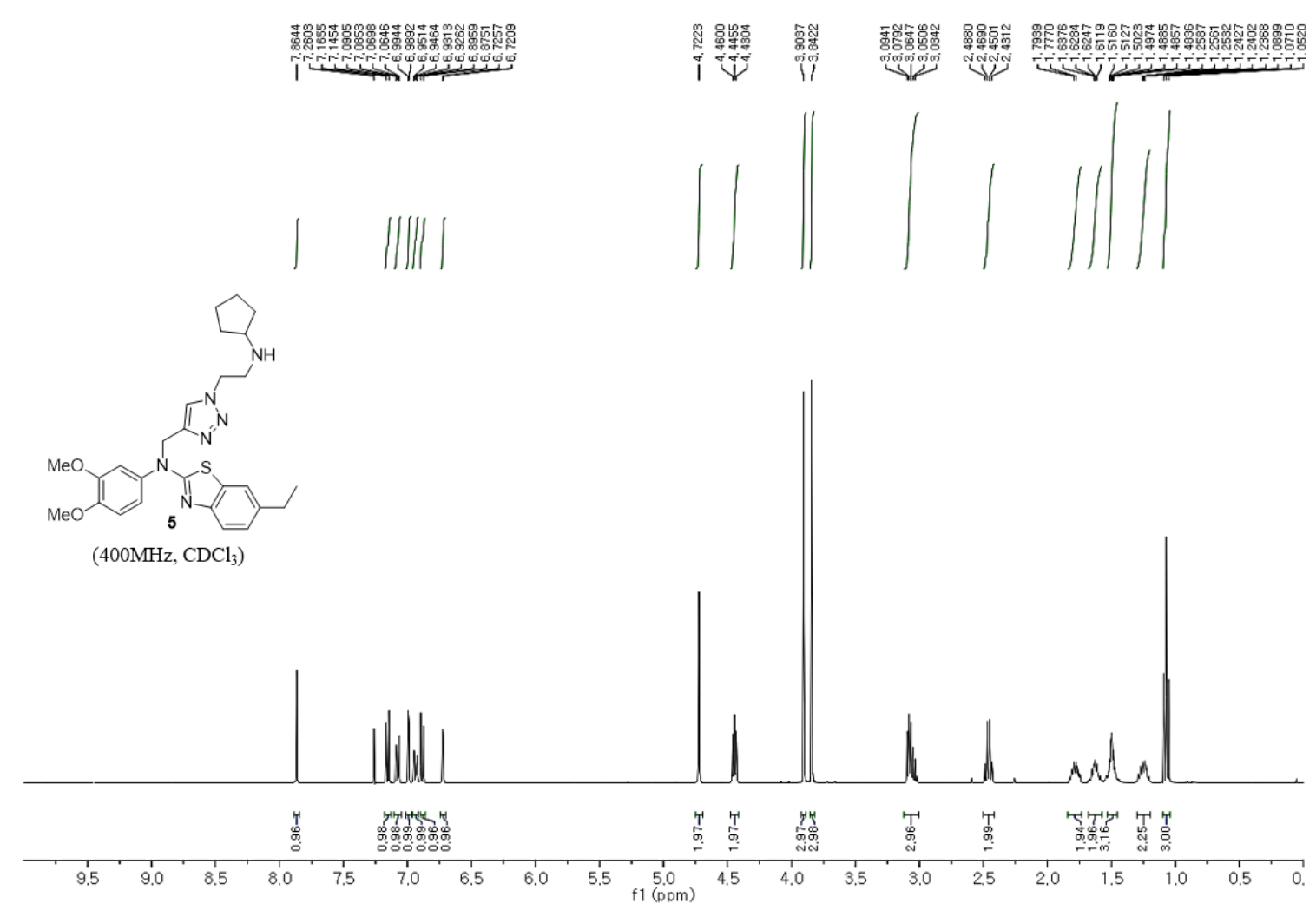

${ }^{1} \mathrm{H}$ NMR spectrum $(400 \mathrm{MHz})$ of 5 in $\mathrm{CDCl}_{3}$

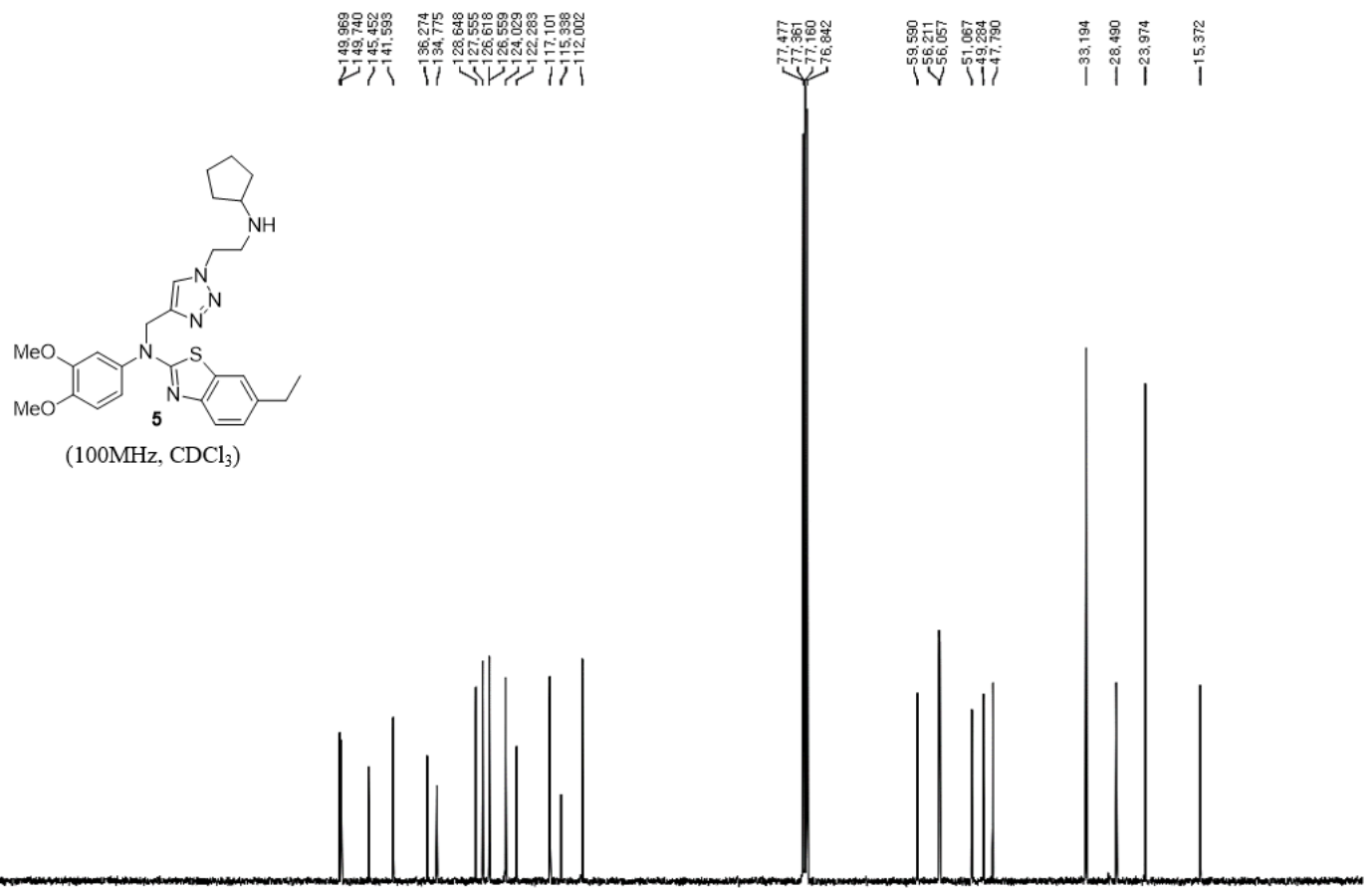

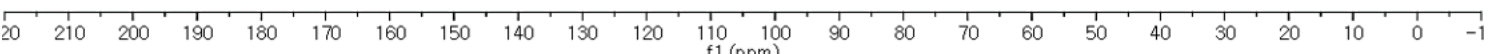

${ }^{13} \mathrm{C}$ NMR spectrum $(100 \mathrm{MHz})$ of 5 in $\mathrm{CDCl}_{3}$ 


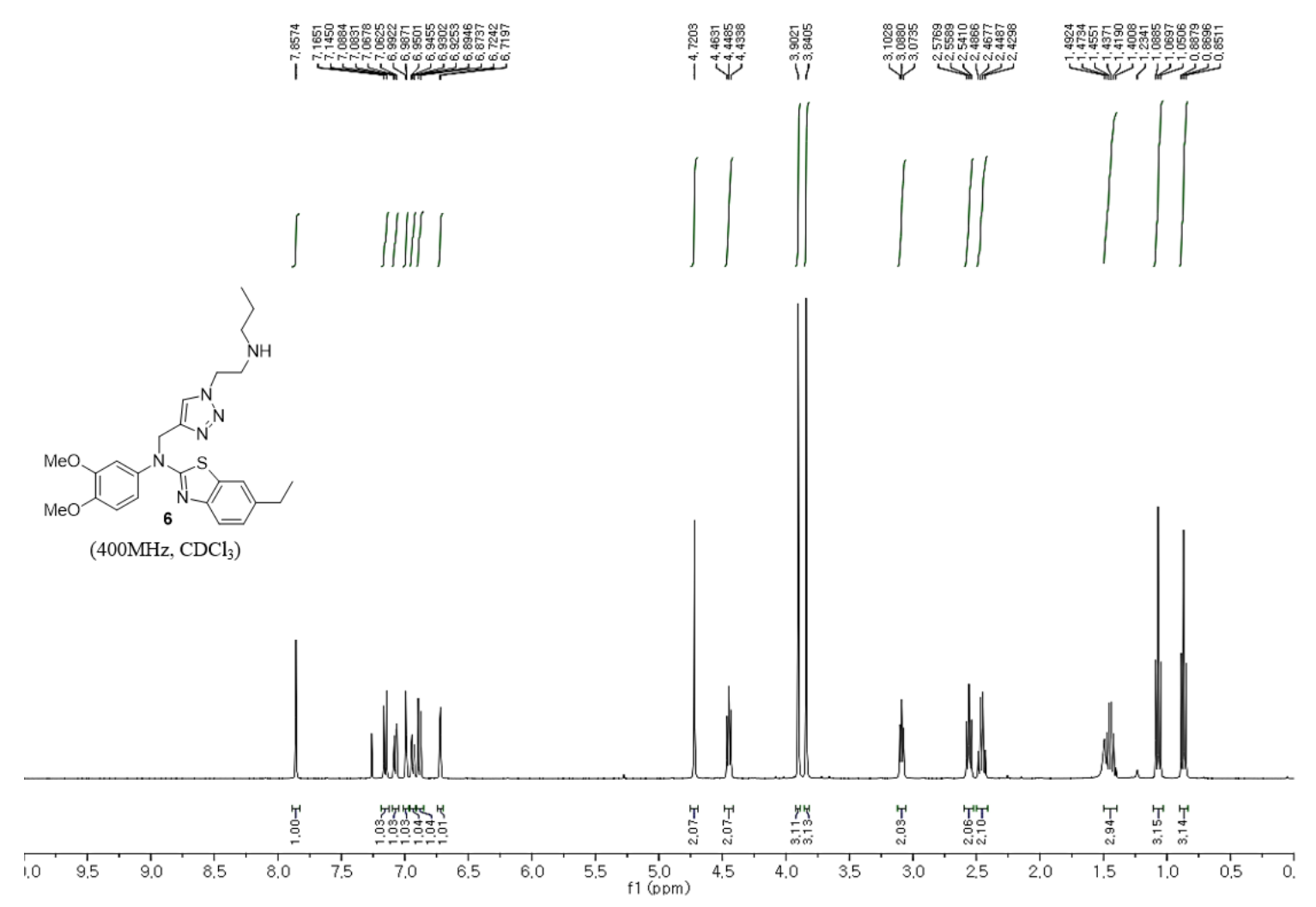

${ }^{1} \mathrm{H}$ NMR spectrum (400 MHz) of 6 in $\mathrm{CDCl}_{3}$

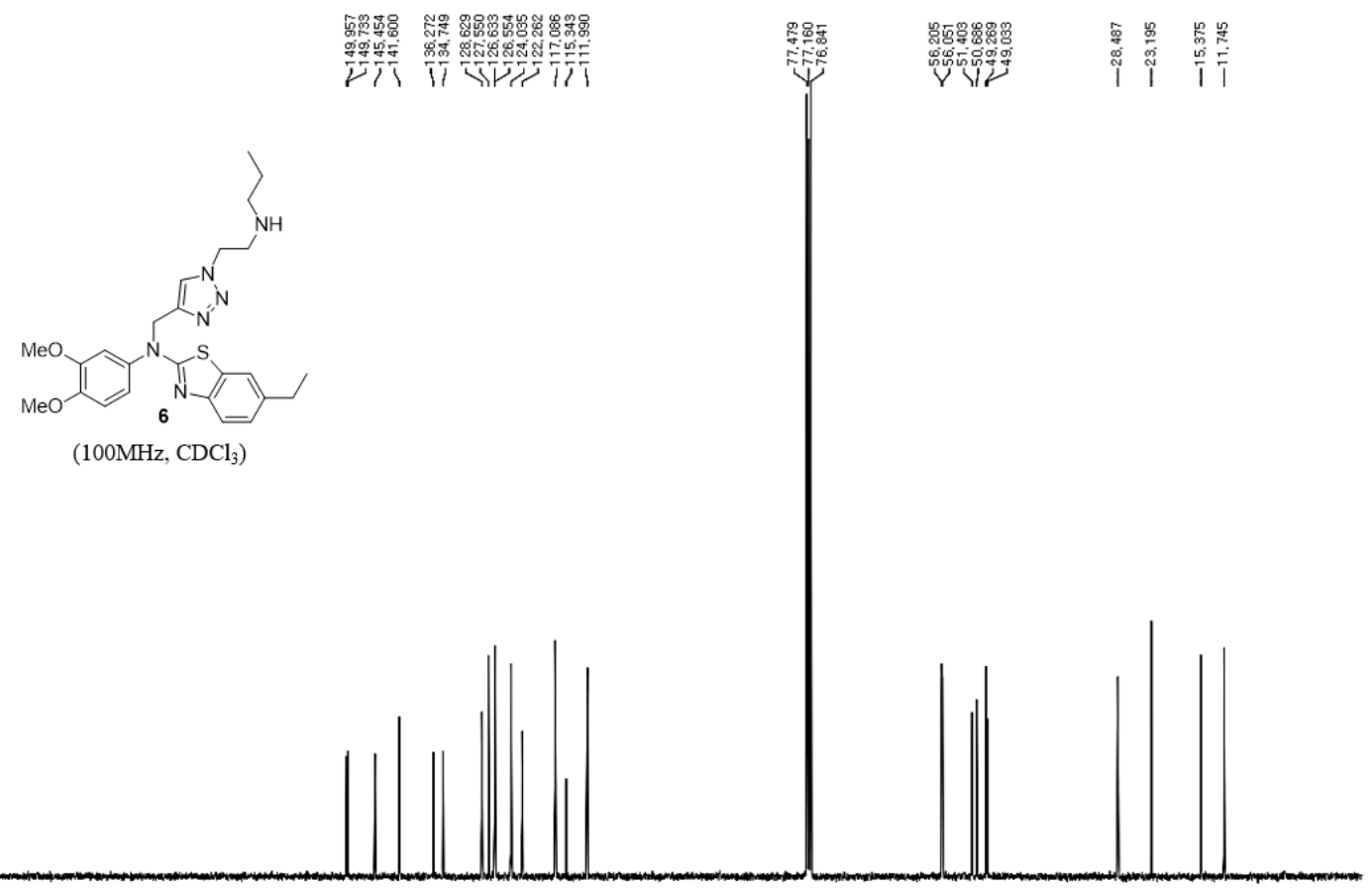

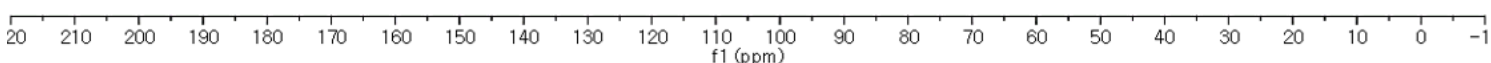

${ }^{13} \mathrm{C}$ NMR spectrum $(100 \mathrm{MHz})$ of 6 in $\mathrm{CDCl}_{3}$ 


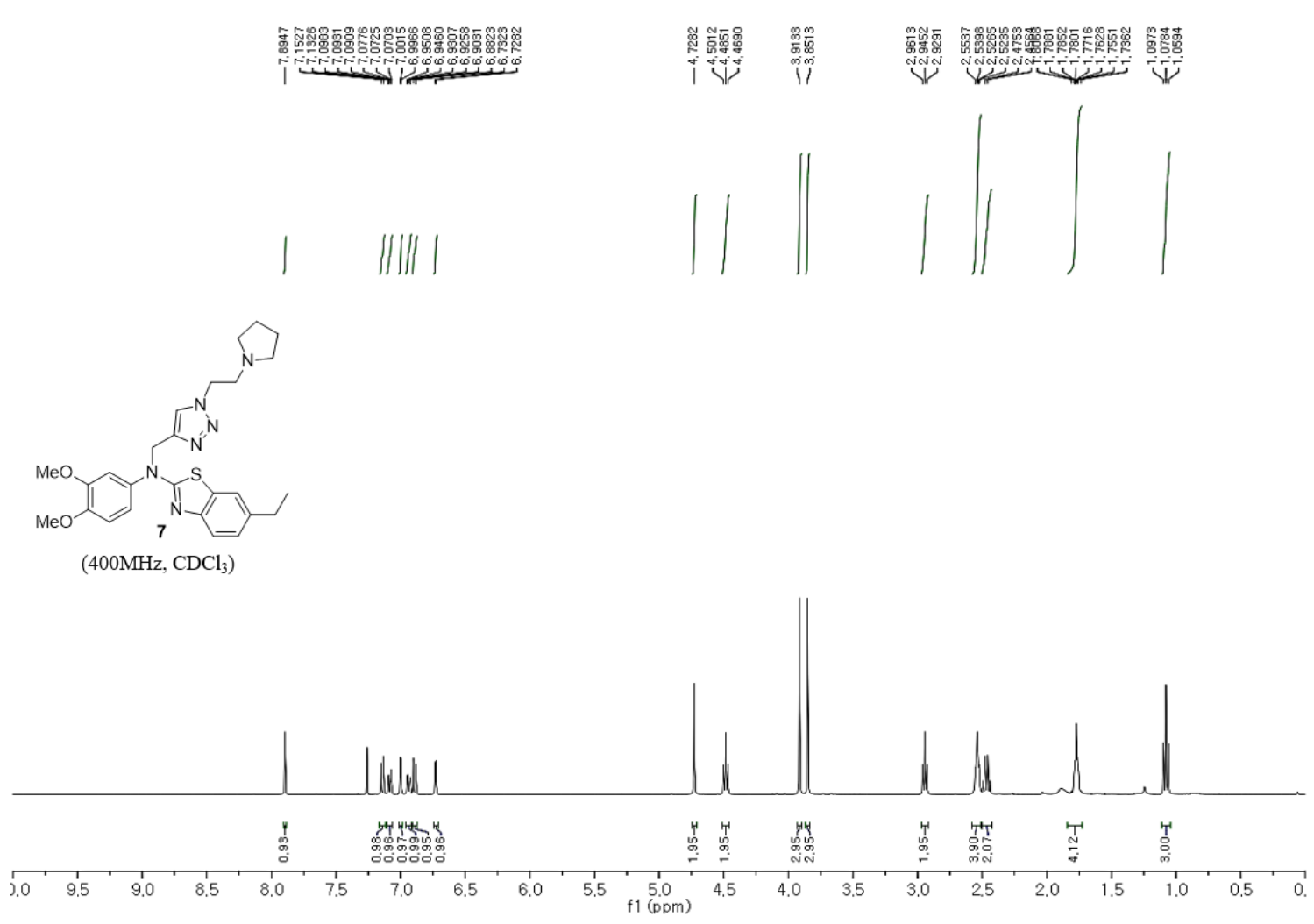

${ }^{1} \mathrm{H}$ NMR spectrum (400 MHz) of 7 in $\mathrm{CDCl}_{3}$

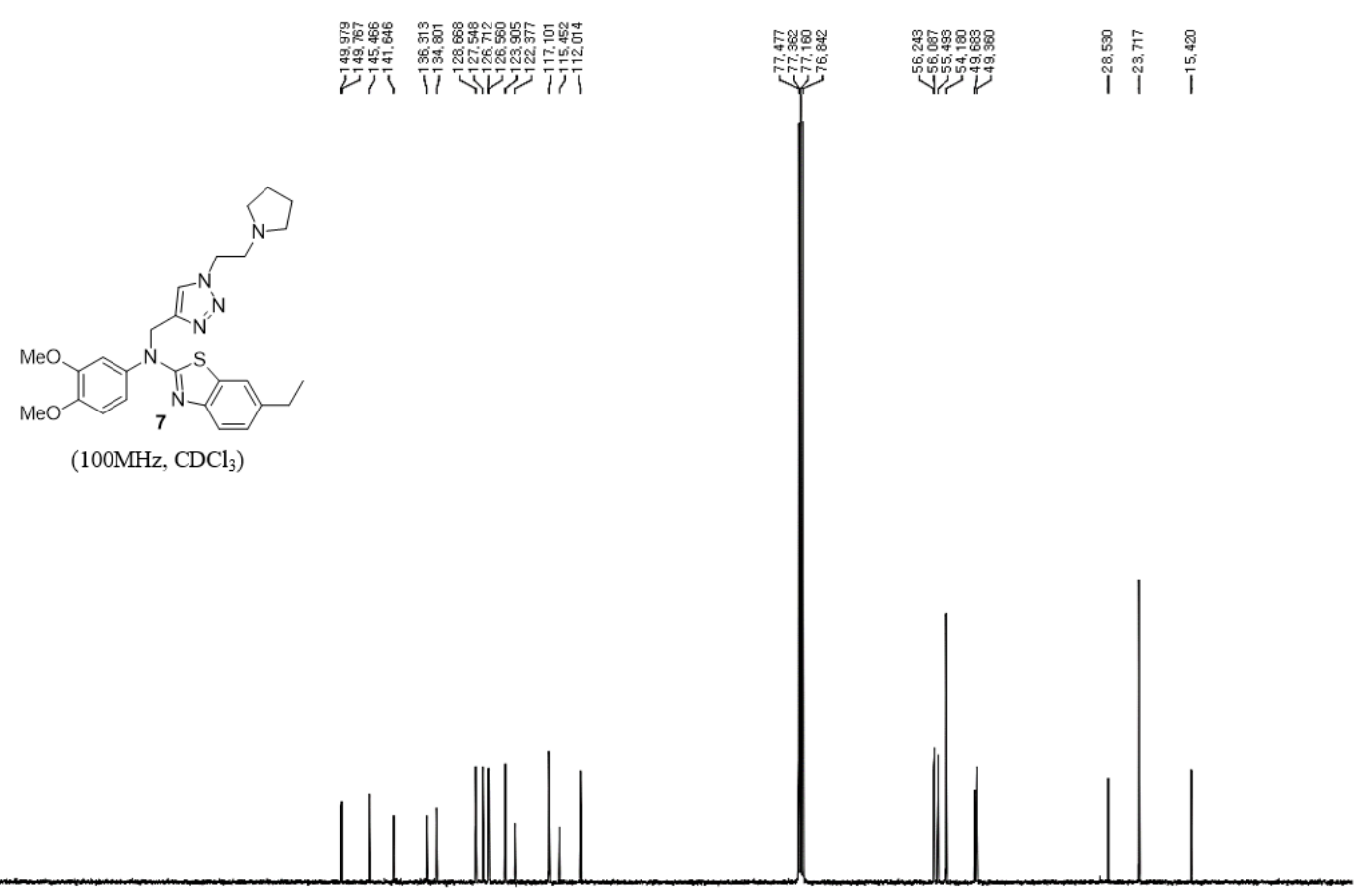

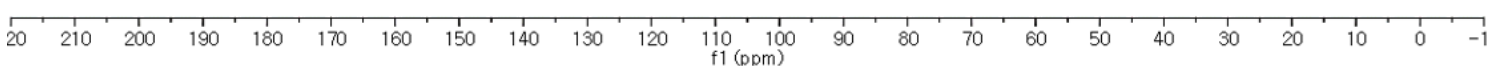

${ }^{13} \mathrm{C}$ NMR spectrum $(100 \mathrm{MHz})$ of 7 in $\mathrm{CDCl}_{3}$ 


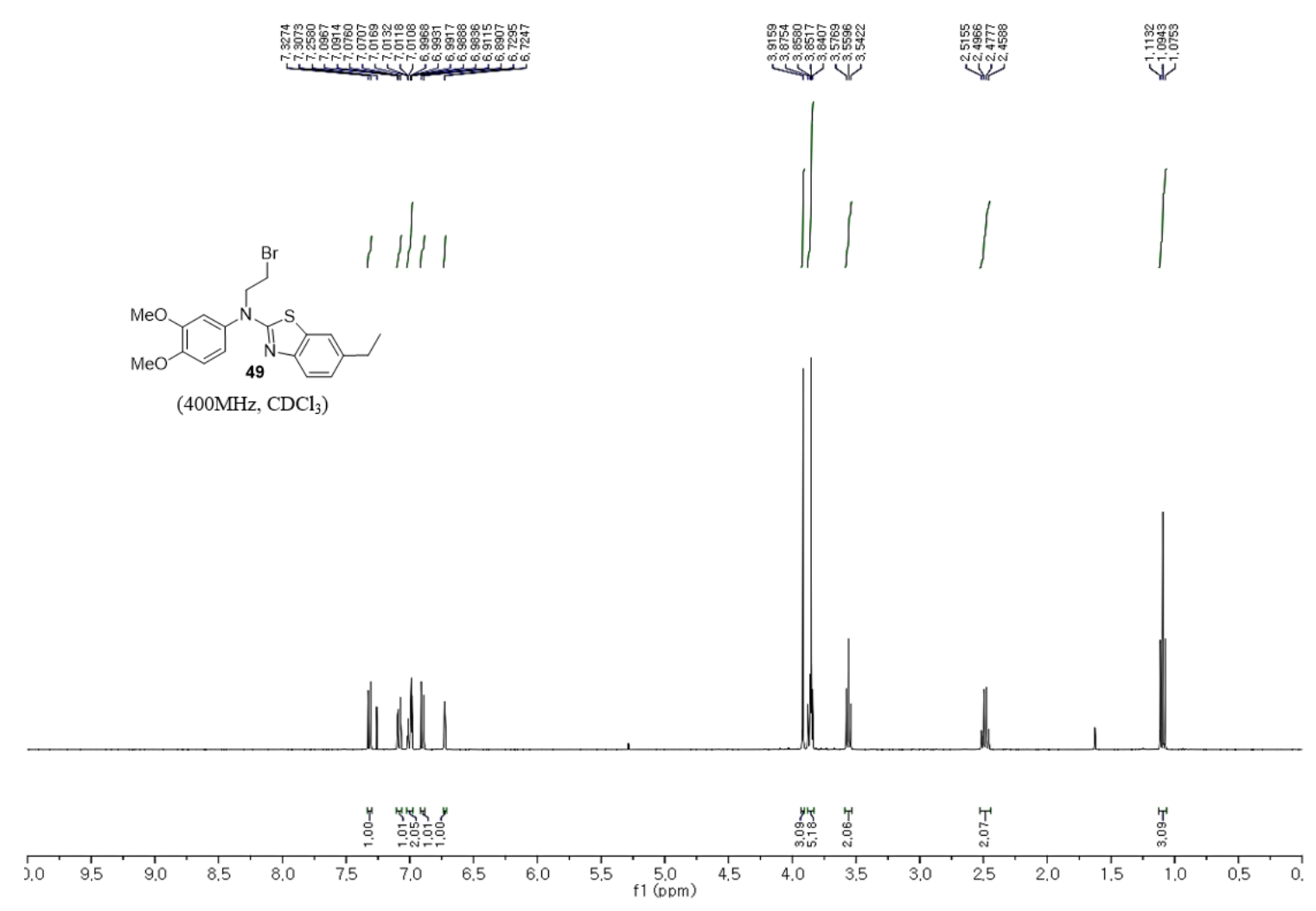

${ }^{1} \mathrm{H}$ NMR spectrum (400 MHz) of 49 in $\mathrm{CDCl}_{3}$
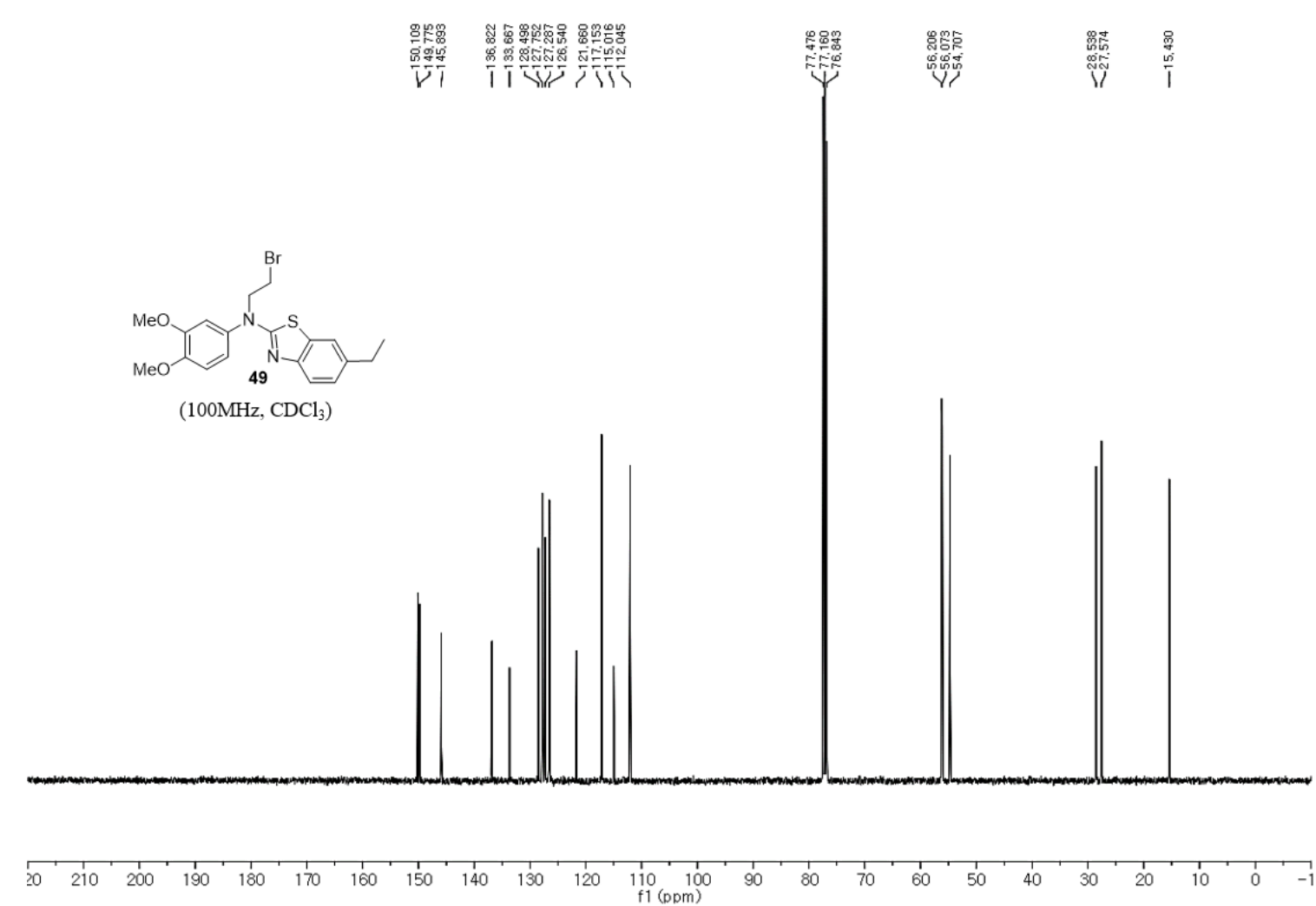

${ }^{13} \mathrm{C}$ NMR spectrum $(100 \mathrm{MHz})$ of 49 in $\mathrm{CDCl}_{3}$ 


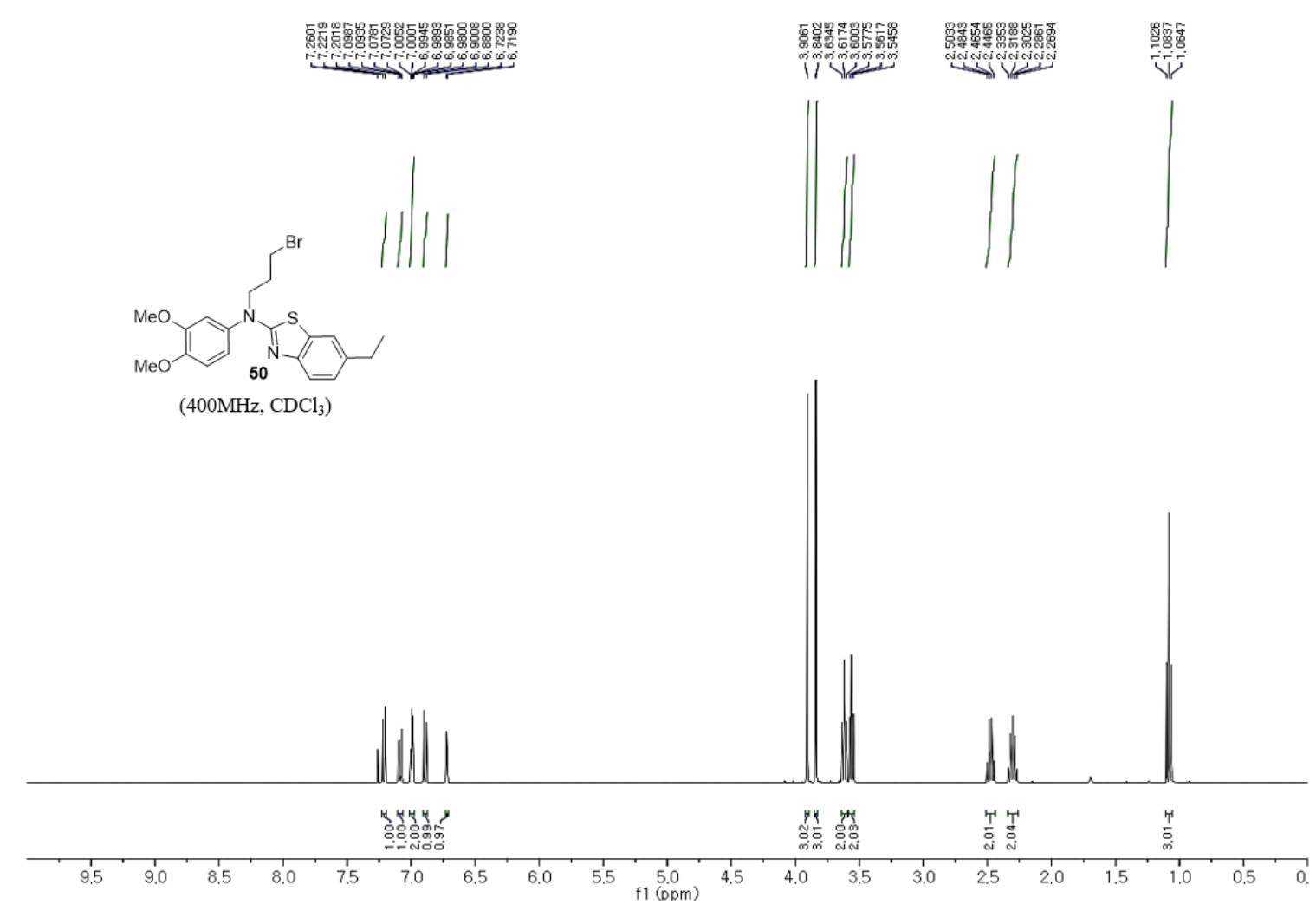

${ }^{1} \mathrm{H}$ NMR spectrum (400 MHz) of $\mathbf{5 0}$ in $\mathrm{CDCl}_{3}$
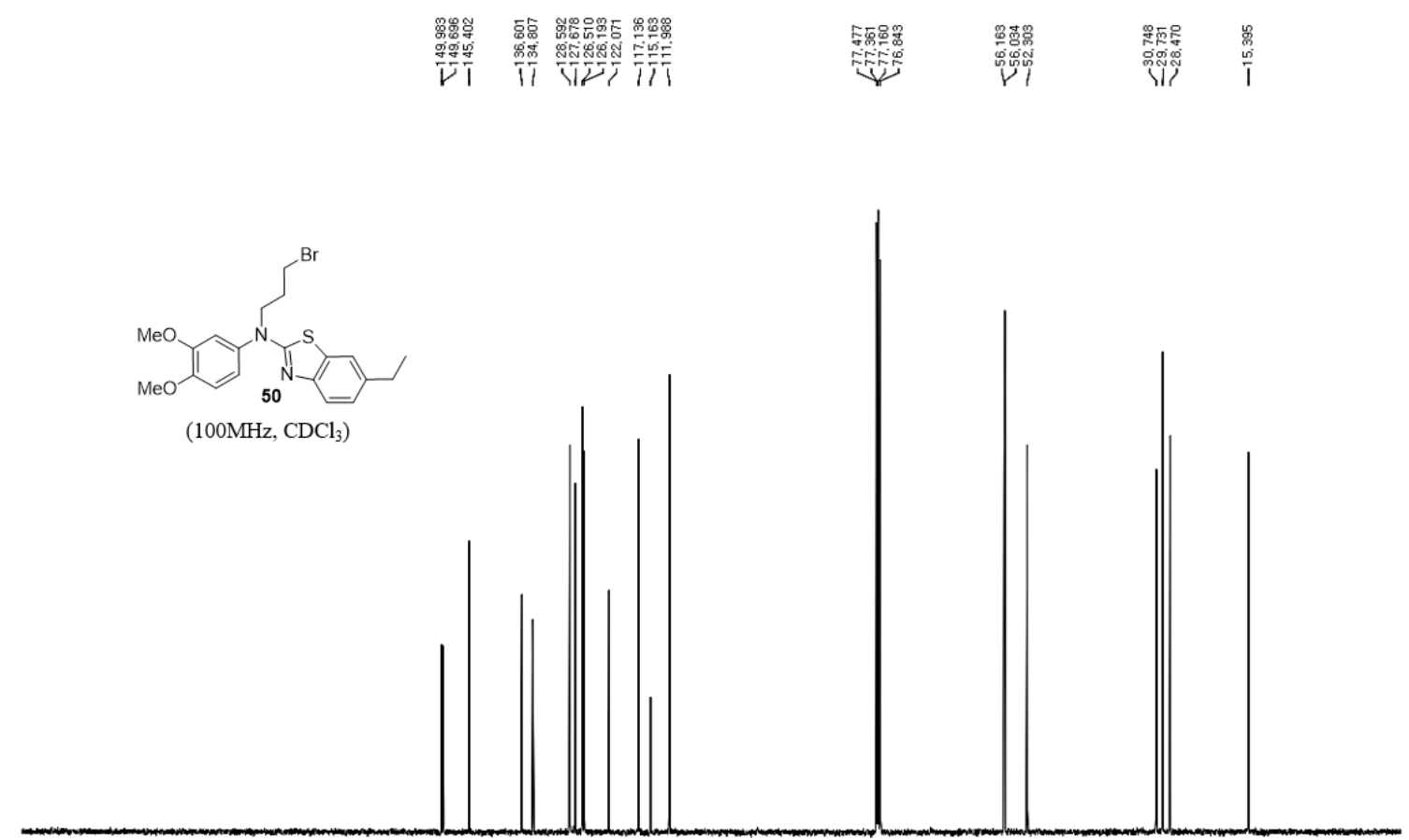

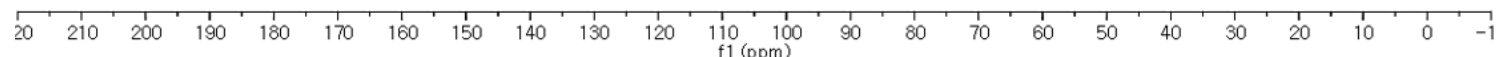

${ }^{13} \mathrm{C}$ NMR spectrum $(100 \mathrm{MHz})$ of $\mathbf{5 0}$ in $\mathrm{CDCl}_{3}$ 


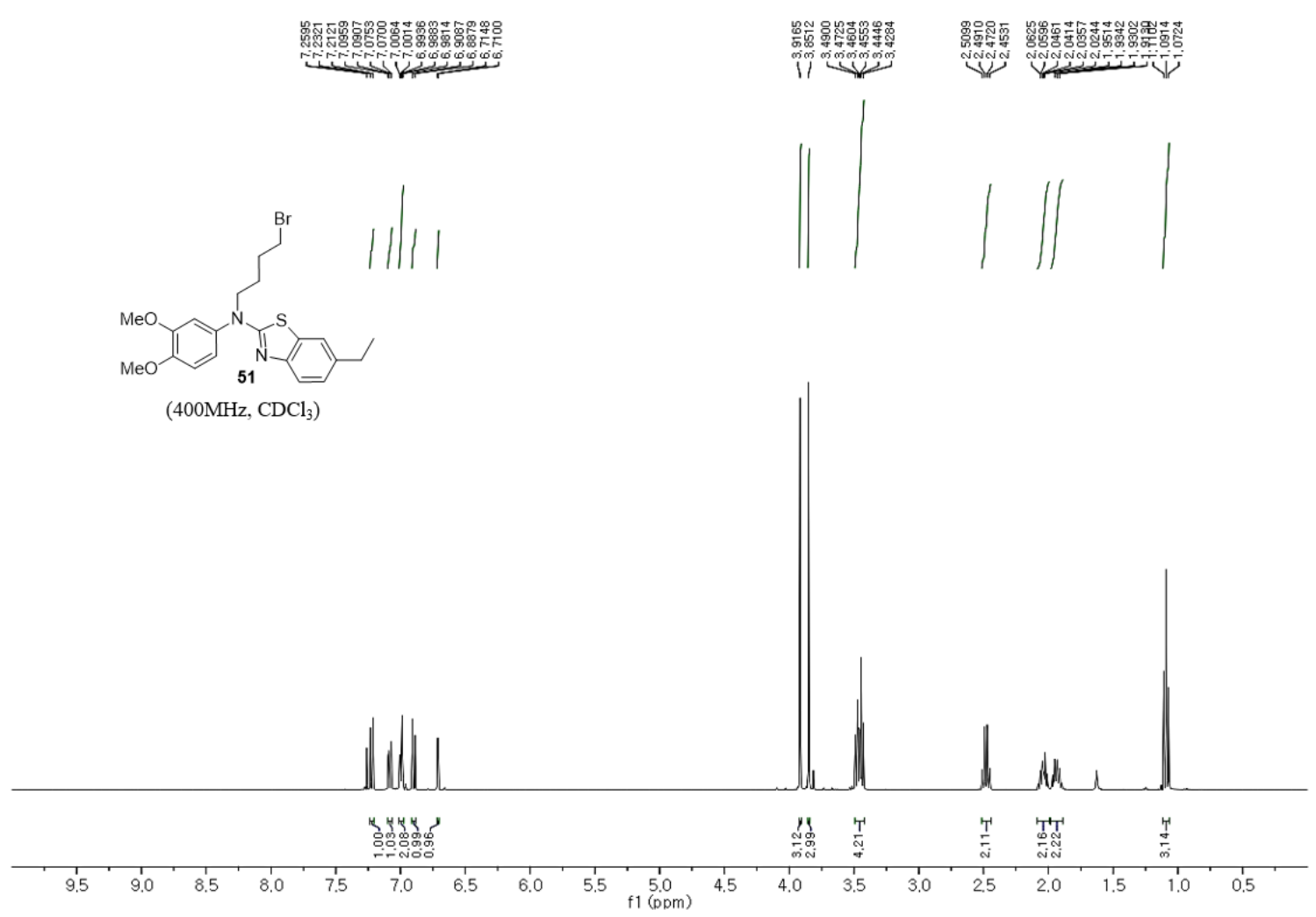

${ }^{1} \mathrm{H}$ NMR spectrum $(400 \mathrm{MHz})$ of $\mathbf{5 1}$ in $\mathrm{CDCl}_{3}$

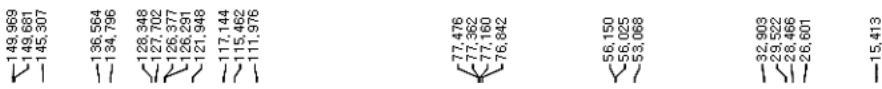
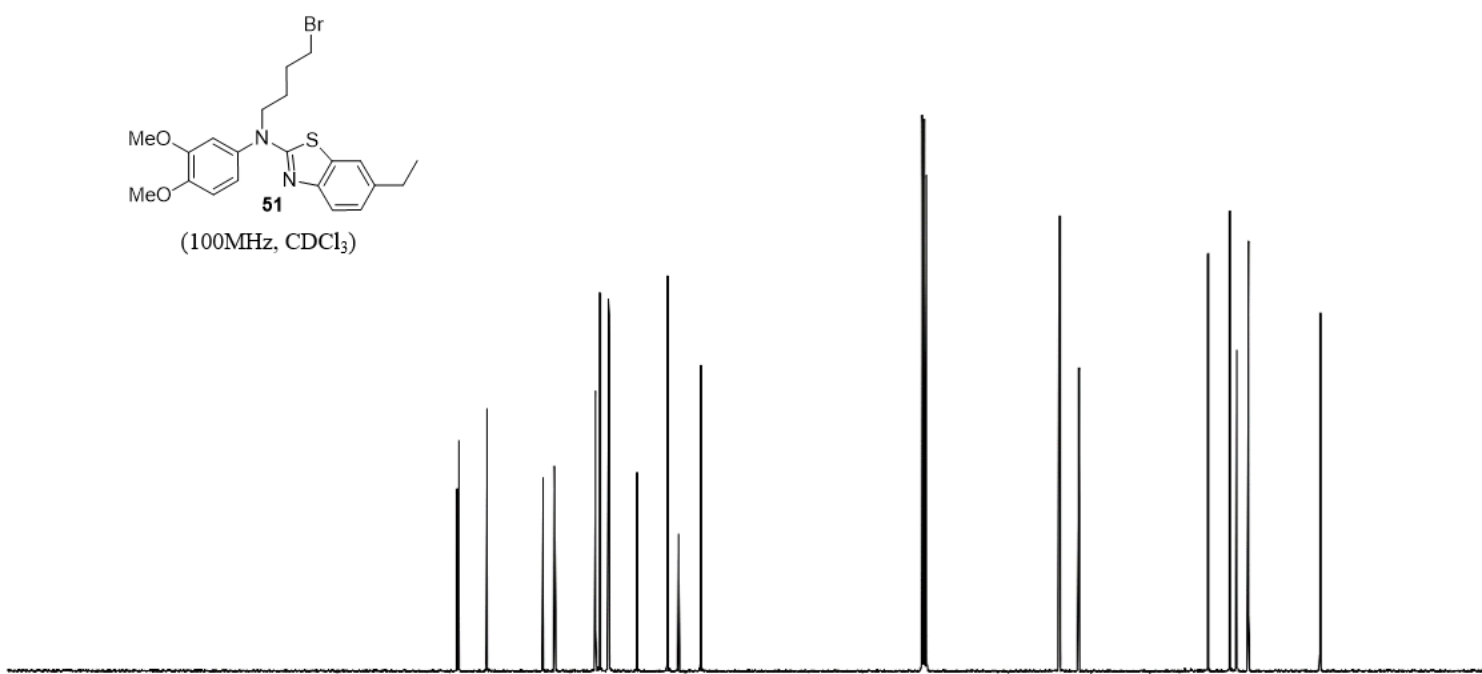

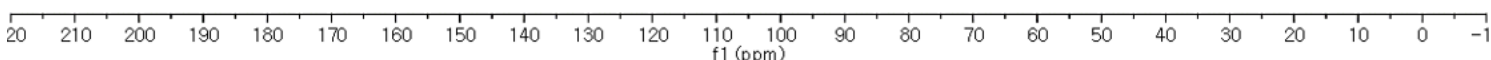

${ }^{13} \mathrm{C}$ NMR spectrum $(100 \mathrm{MHz})$ of 51 in $\mathrm{CDCl}_{3}$ 

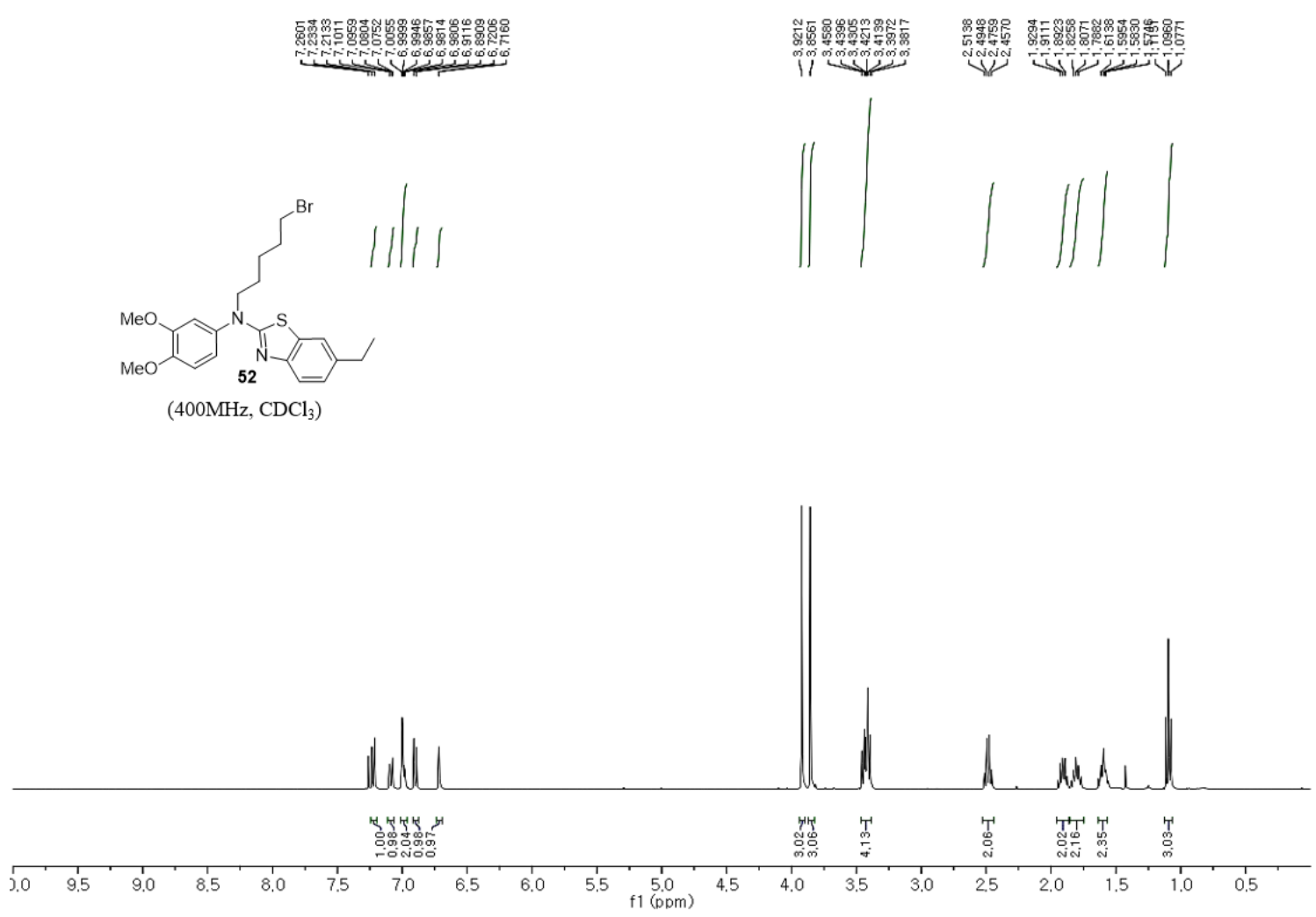

${ }^{1} \mathrm{H}$ NMR spectrum $(400 \mathrm{MHz})$ of $\mathbf{5 2}$ in $\mathrm{CDCl}_{3}$

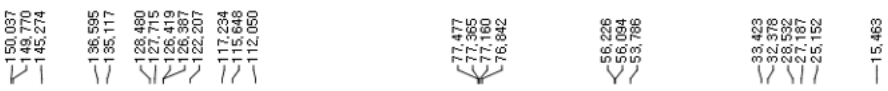

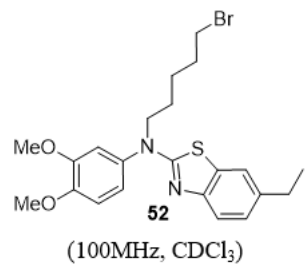

$\left(100 \mathrm{MHz}, \mathrm{CDCl}_{3}\right)$

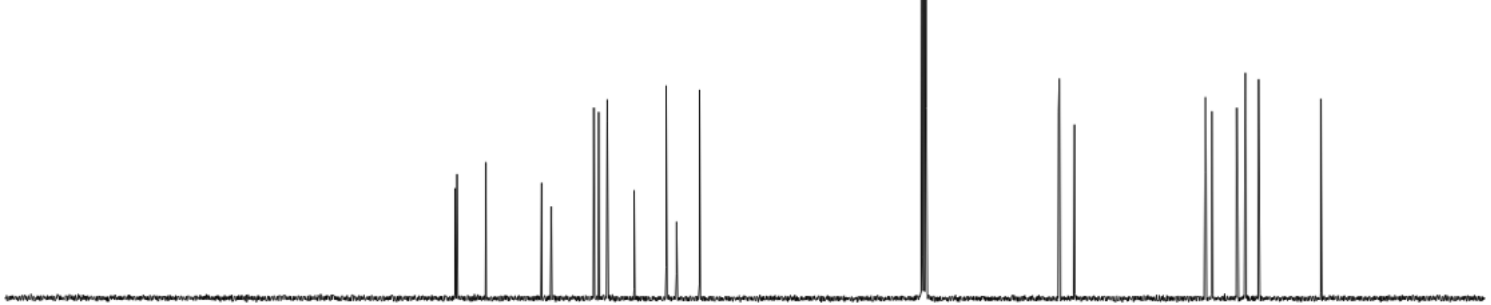

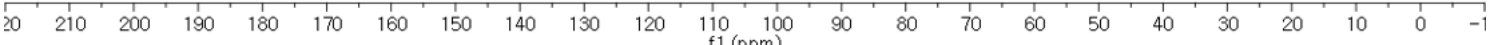

${ }^{13} \mathrm{C}$ NMR spectrum $(100 \mathrm{MHz})$ of $\mathbf{5 2}$ in $\mathrm{CDCl}_{3}$ 

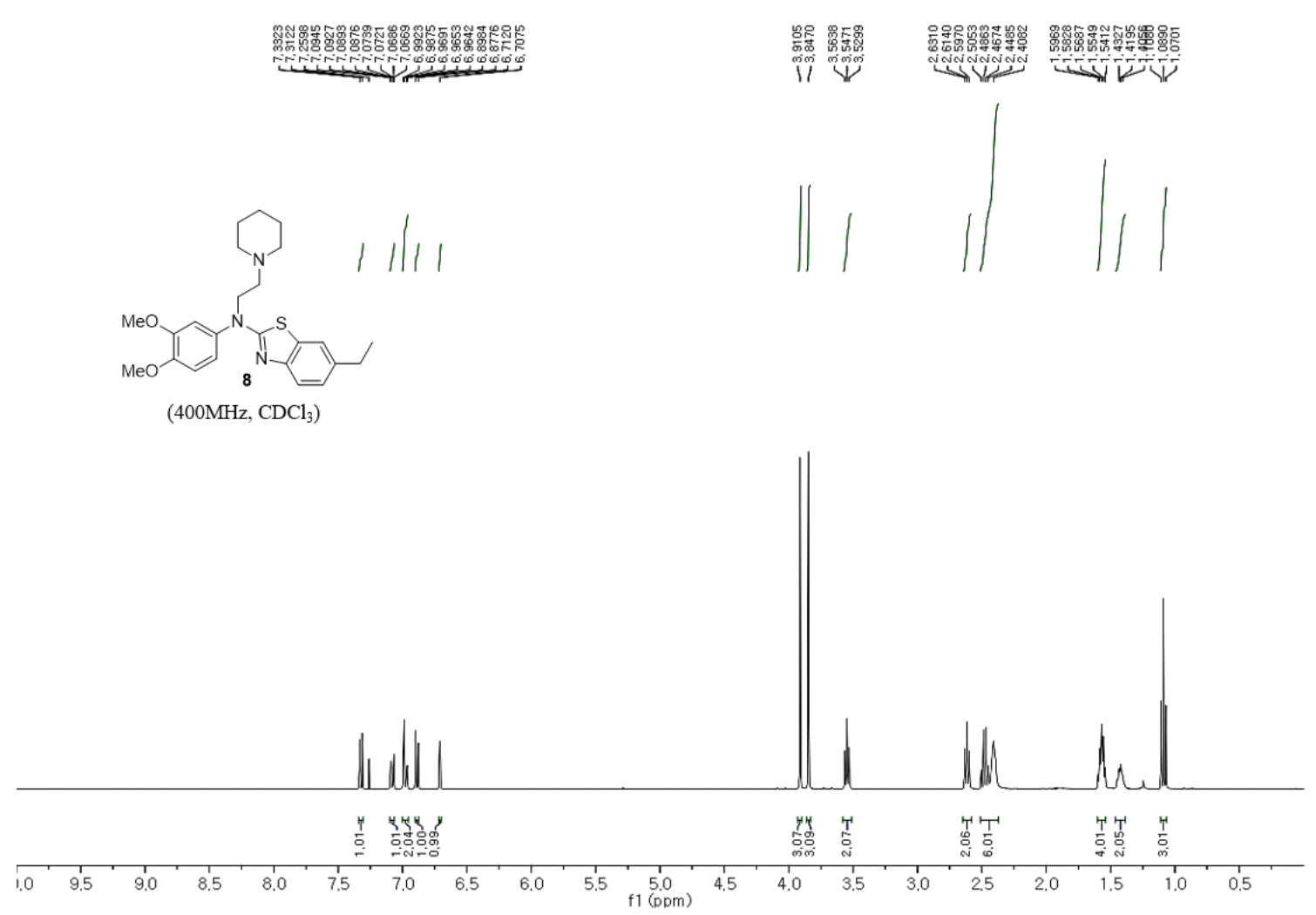

${ }^{1} \mathrm{H}$ NMR spectrum $(400 \mathrm{MHz})$ of $\mathbf{8}$ in $\mathrm{CDCl}_{3}$

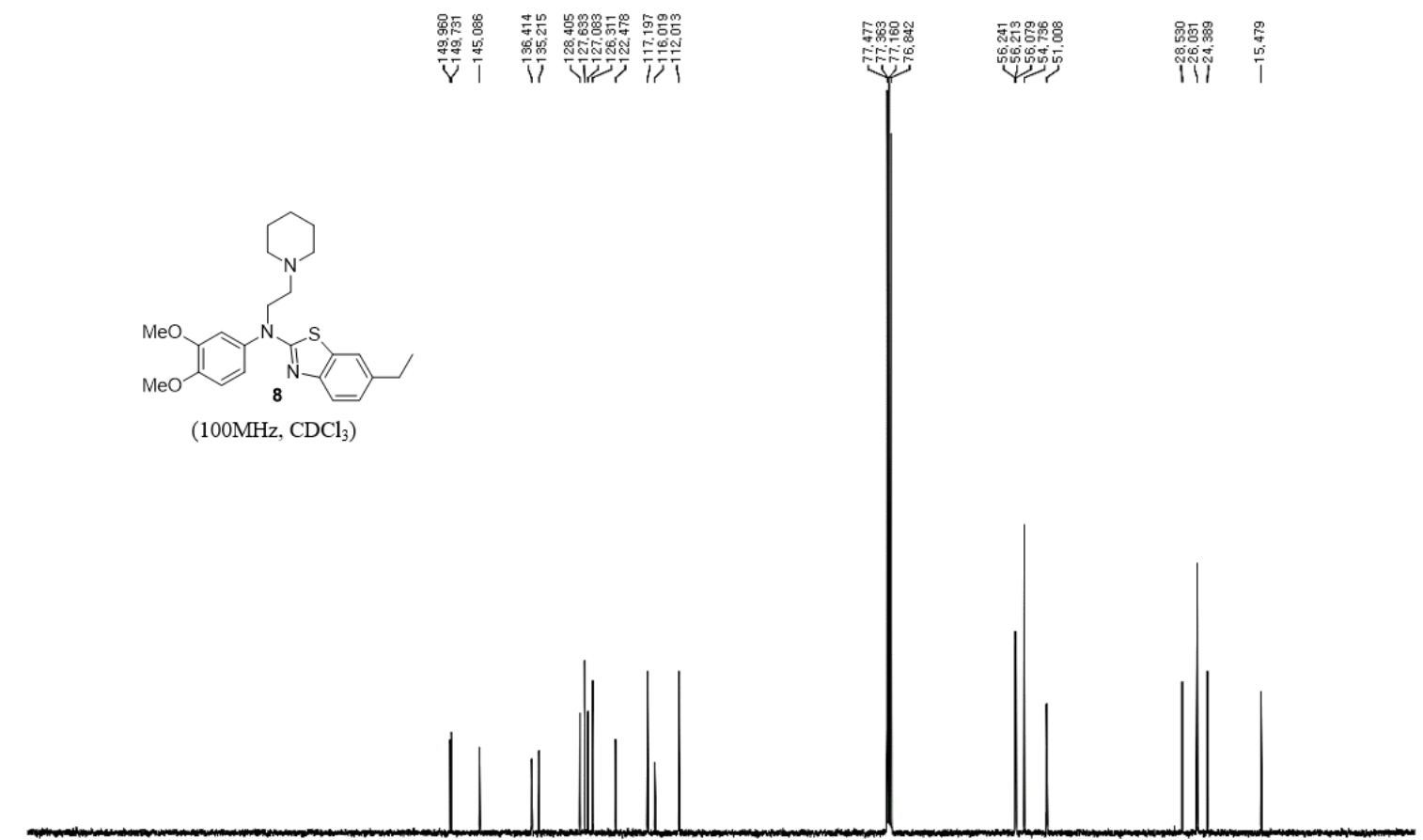

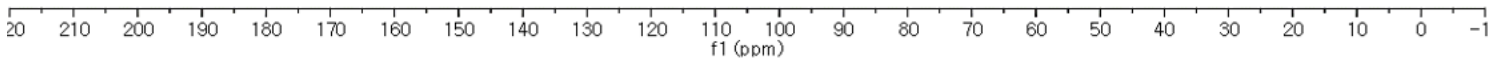

${ }^{13} \mathrm{C}$ NMR spectrum $(100 \mathrm{MHz})$ of 8 in $\mathrm{CDCl}_{3}$ 

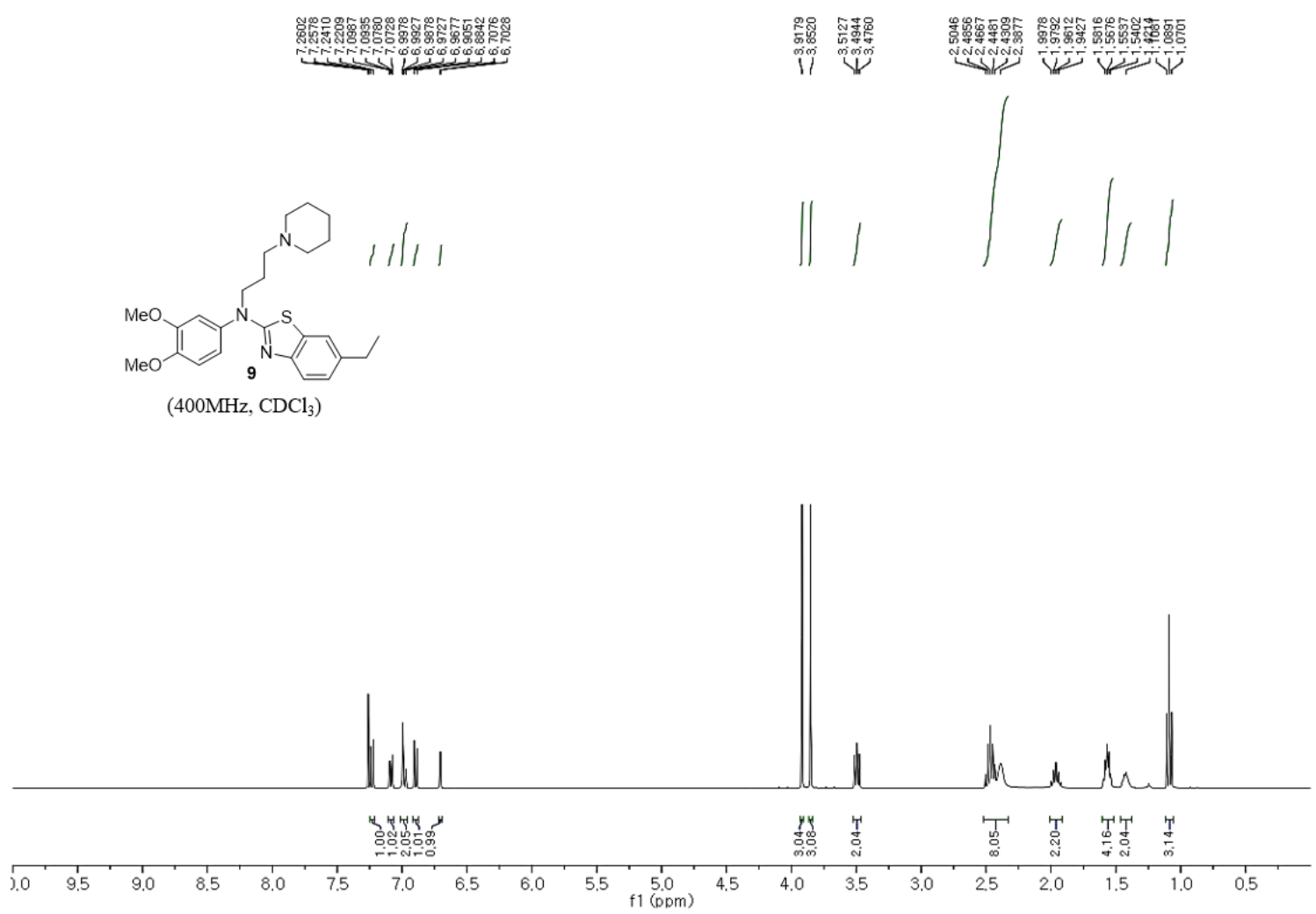

${ }^{1} \mathrm{H}$ NMR spectrum $(400 \mathrm{MHz})$ of 9 in $\mathrm{CDCl}_{3}$

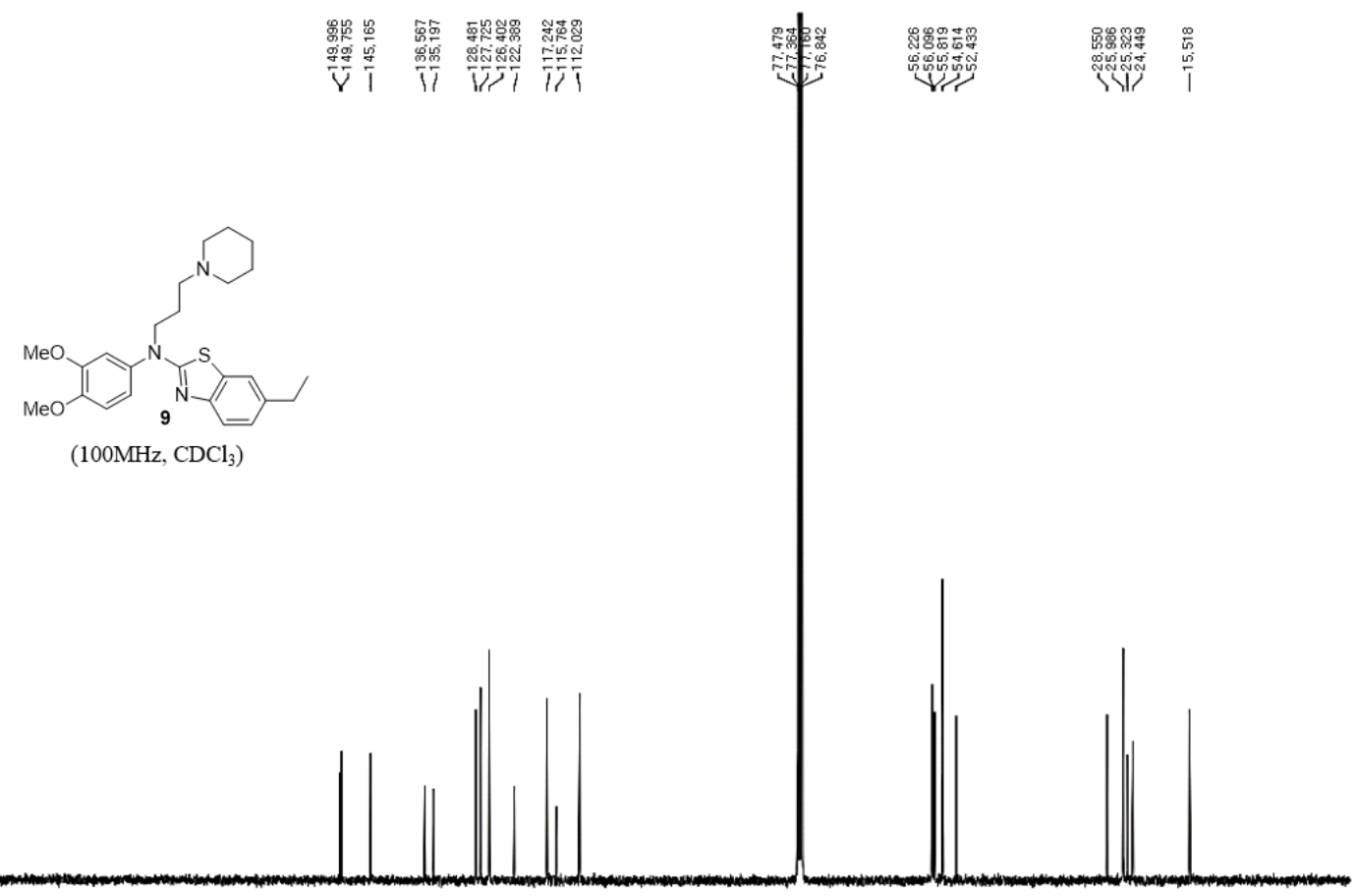

$\begin{array}{llllllllllll}210 & 200 & 190 & 180 & 170 & 160 & 150 & 140 & 130 & 120 & 110 & 100 \\ f 1(\mathrm{pmm})\end{array}$

${ }^{13} \mathrm{C}$ NMR spectrum $(100 \mathrm{MHz})$ of 9 in $\mathrm{CDCl}_{3}$ 

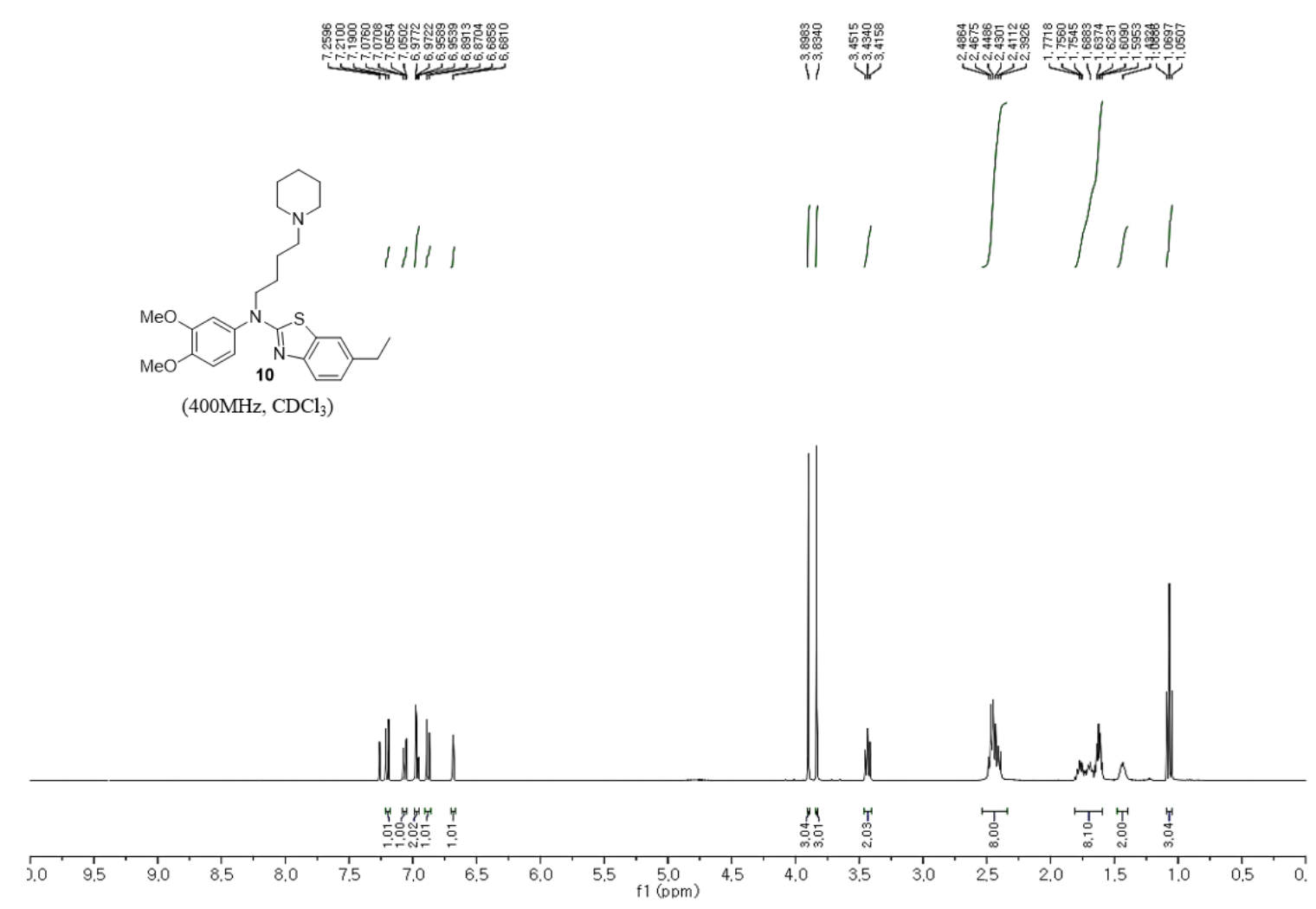

${ }^{1} \mathrm{H}$ NMR spectrum (400 MHz) of $\mathbf{1 0}$ in $\mathrm{CDCl}_{3}$

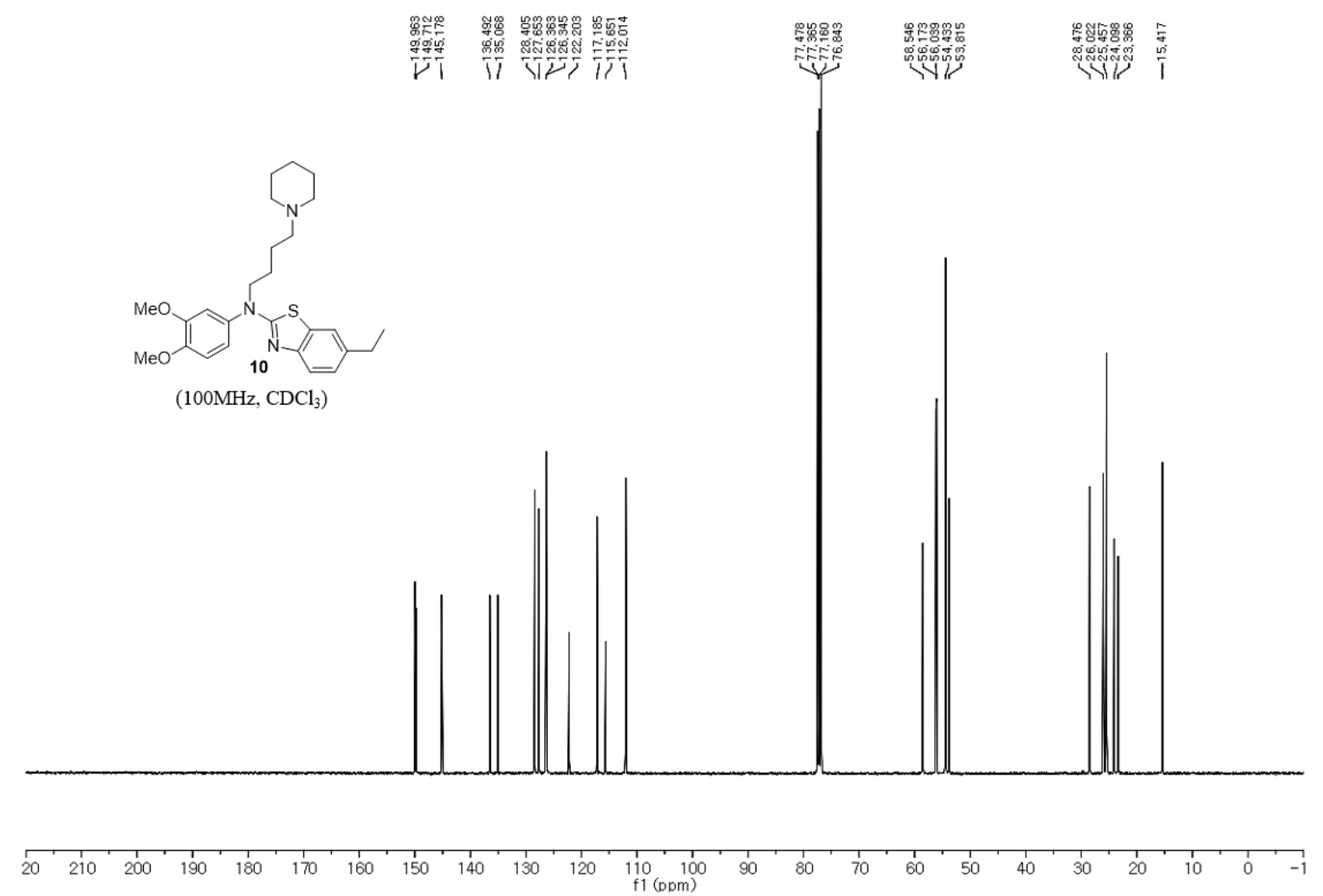

${ }^{13} \mathrm{C}$ NMR spectrum $(100 \mathrm{MHz})$ of $\mathbf{1 0}$ in $\mathrm{CDCl}_{3}$ 

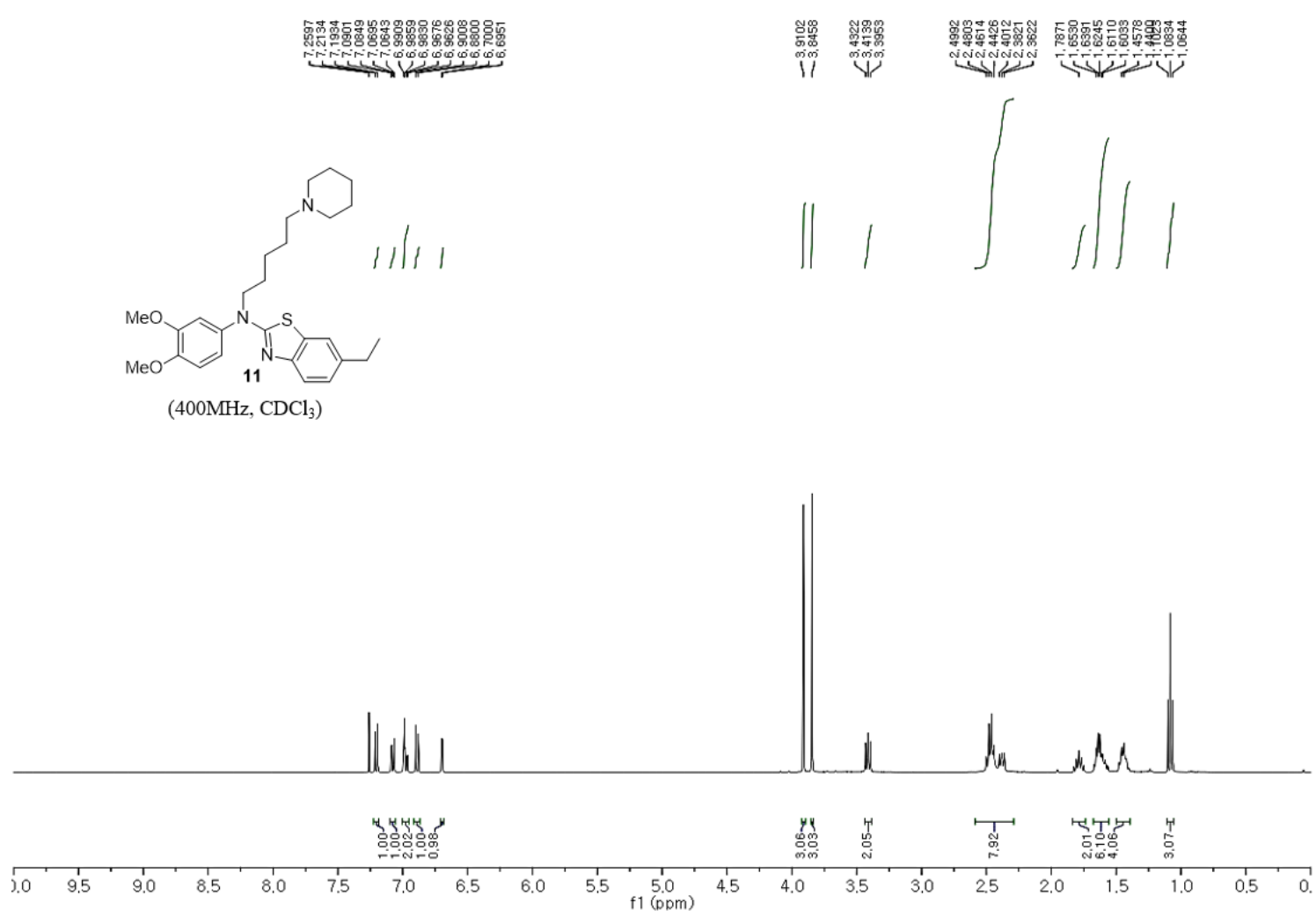

${ }^{1} \mathrm{H}$ NMR spectrum $\left(400 \mathrm{MHz}\right.$ ) of $\mathbf{1 1}$ in $\mathrm{CDCl}_{3}$

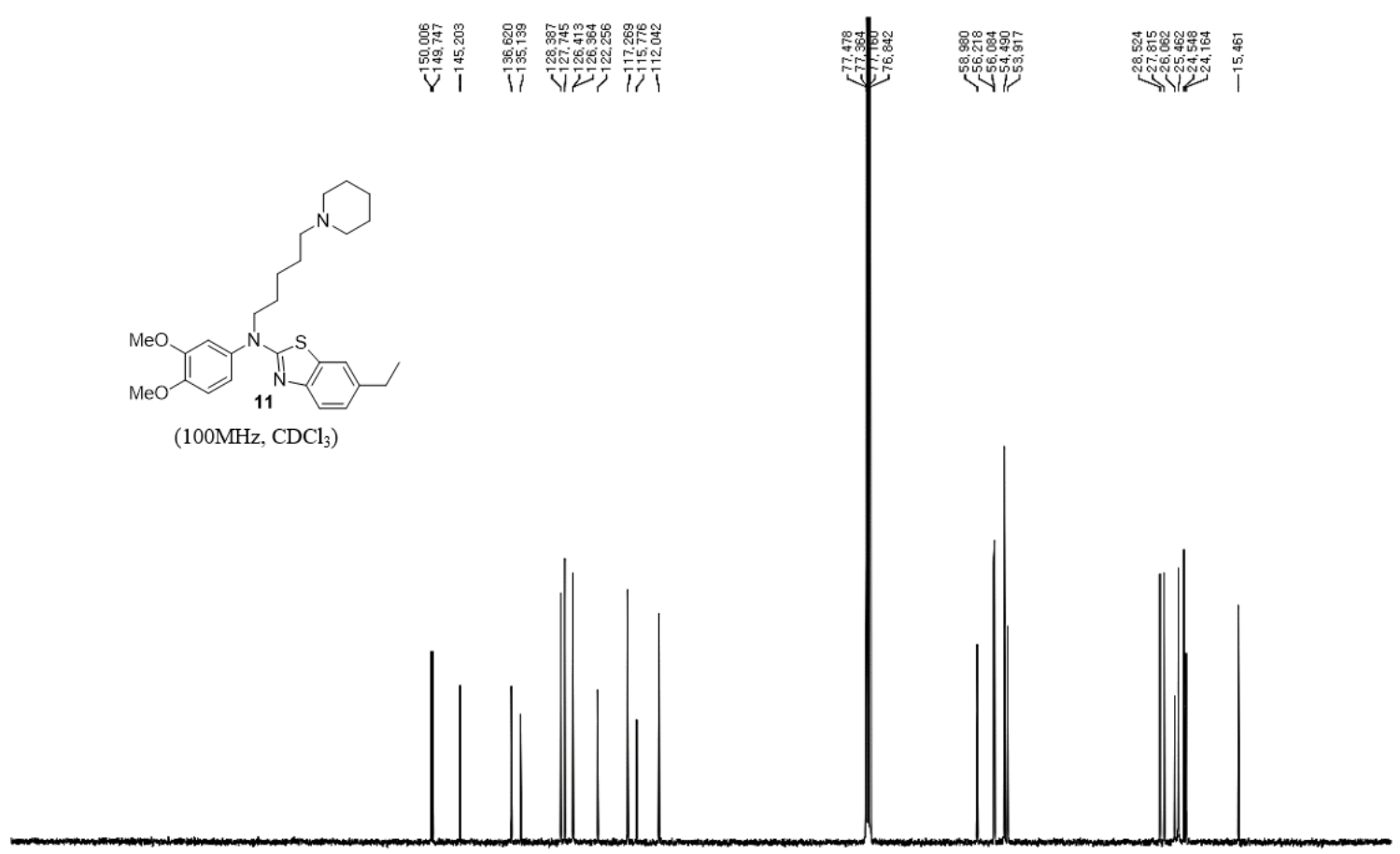

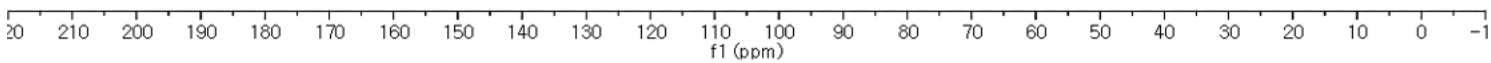

${ }^{13} \mathrm{C}$ NMR spectrum $(100 \mathrm{MHz})$ of $\mathbf{1 1}$ in $\mathrm{CDCl}_{3}$ 

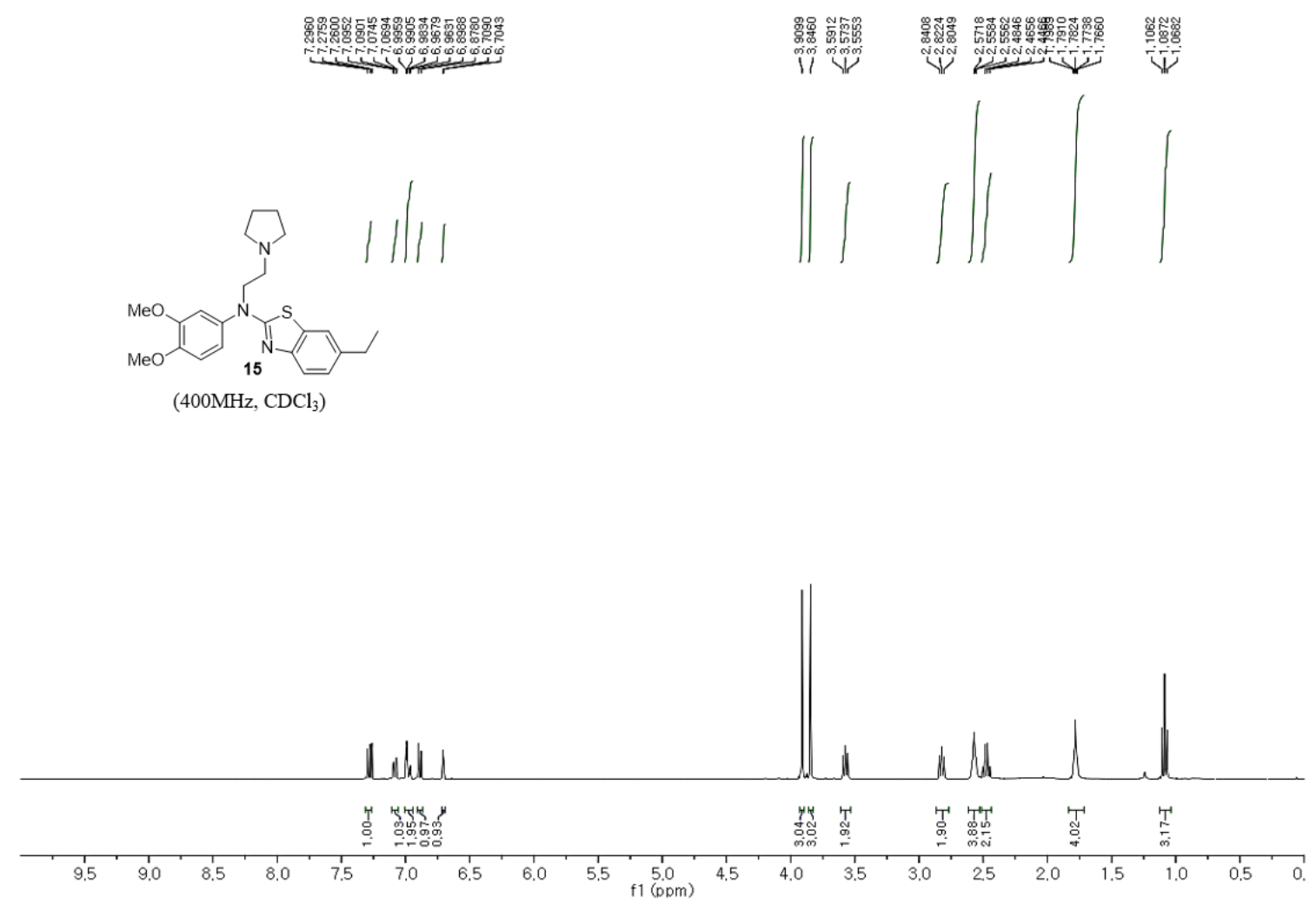

${ }^{1} \mathrm{H}$ NMR spectrum $(400 \mathrm{MHz})$ of 15 in $\mathrm{CDCl}_{3}$

\begin{tabular}{|c|c|c|c|}
\hline 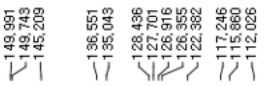 & 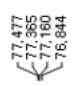 & 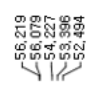 & 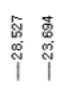 \\
\hline
\end{tabular}
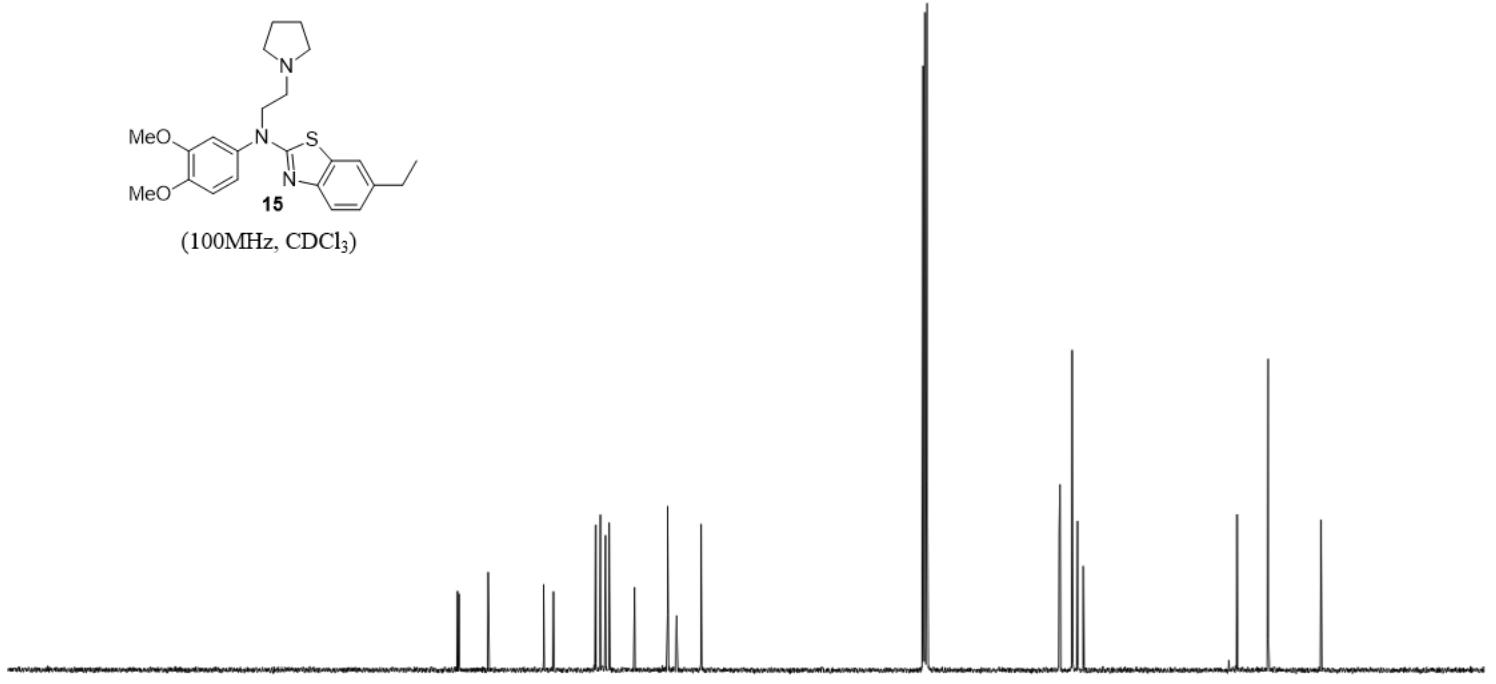

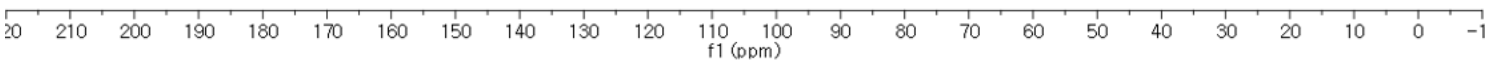

${ }^{13} \mathrm{C}$ NMR spectrum $(100 \mathrm{MHz})$ of 15 in $\mathrm{CDCl}_{3}$ 


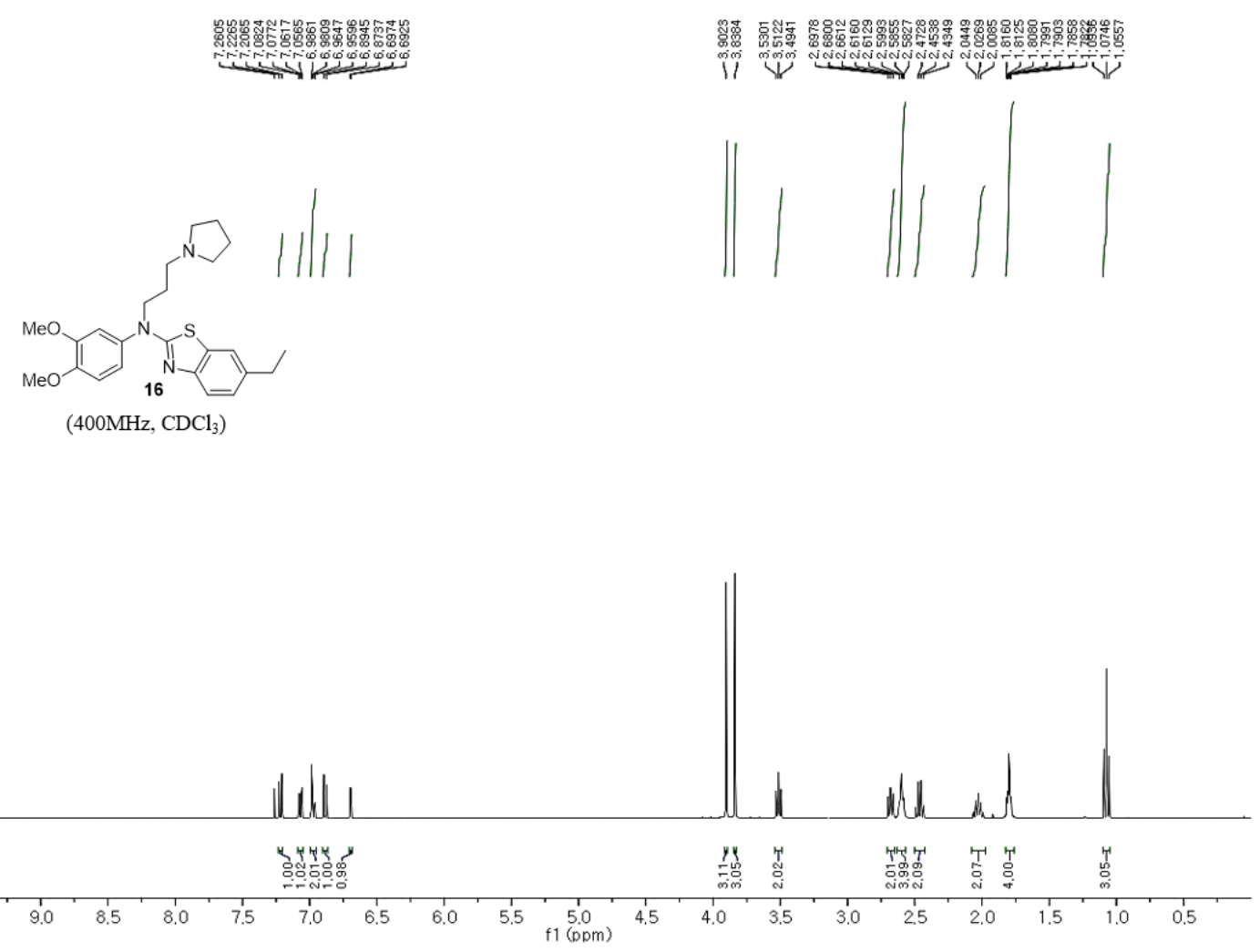

${ }^{1} \mathrm{H}$ NMR spectrum $(400 \mathrm{MHz})$ of $\mathbf{1 6}$ in $\mathrm{CDCl}_{3}$

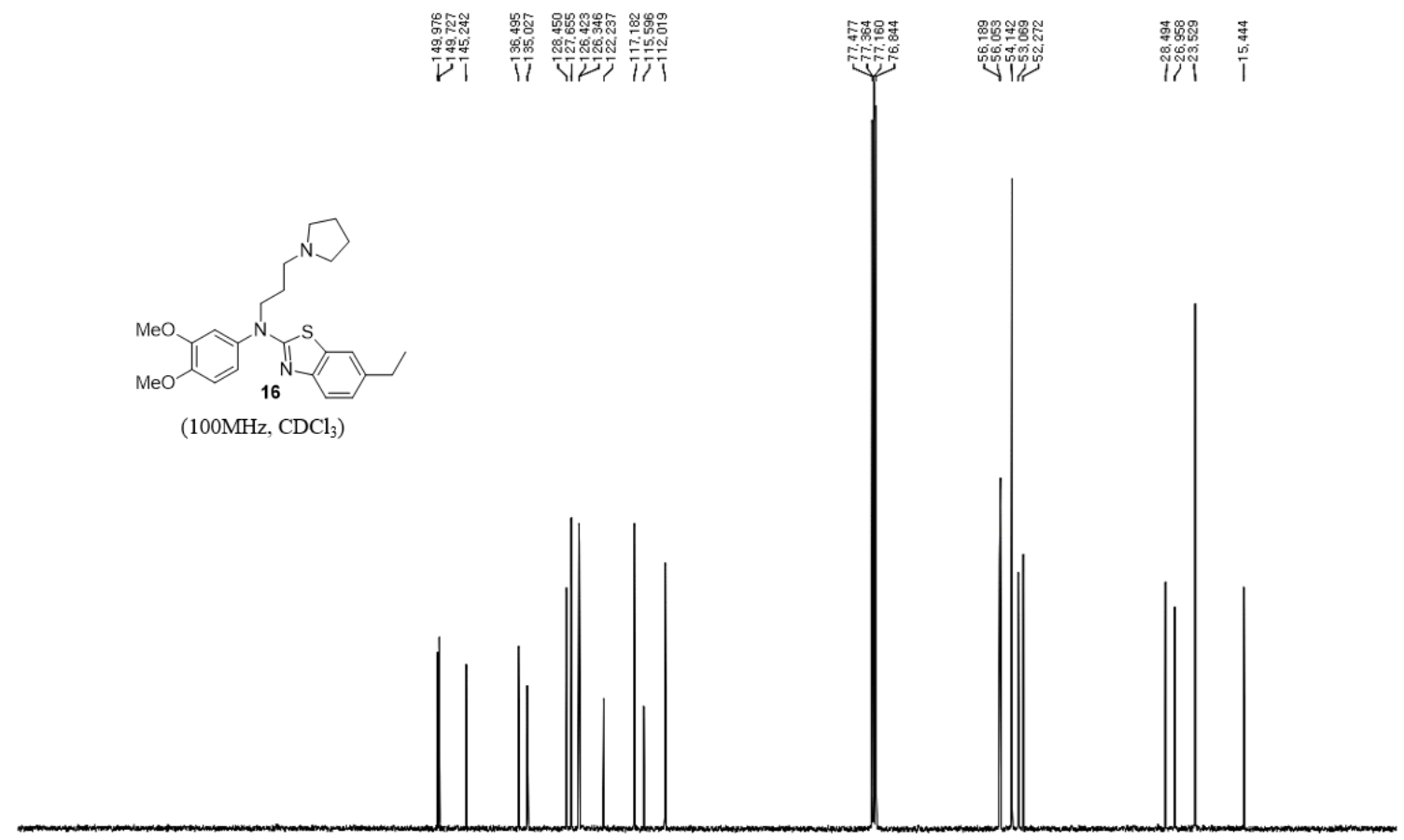

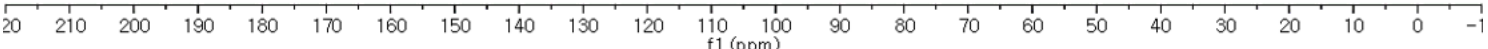

${ }^{13} \mathrm{C}$ NMR spectrum $(100 \mathrm{MHz})$ of $\mathbf{1 6}$ in $\mathrm{CDCl}_{3}$ 

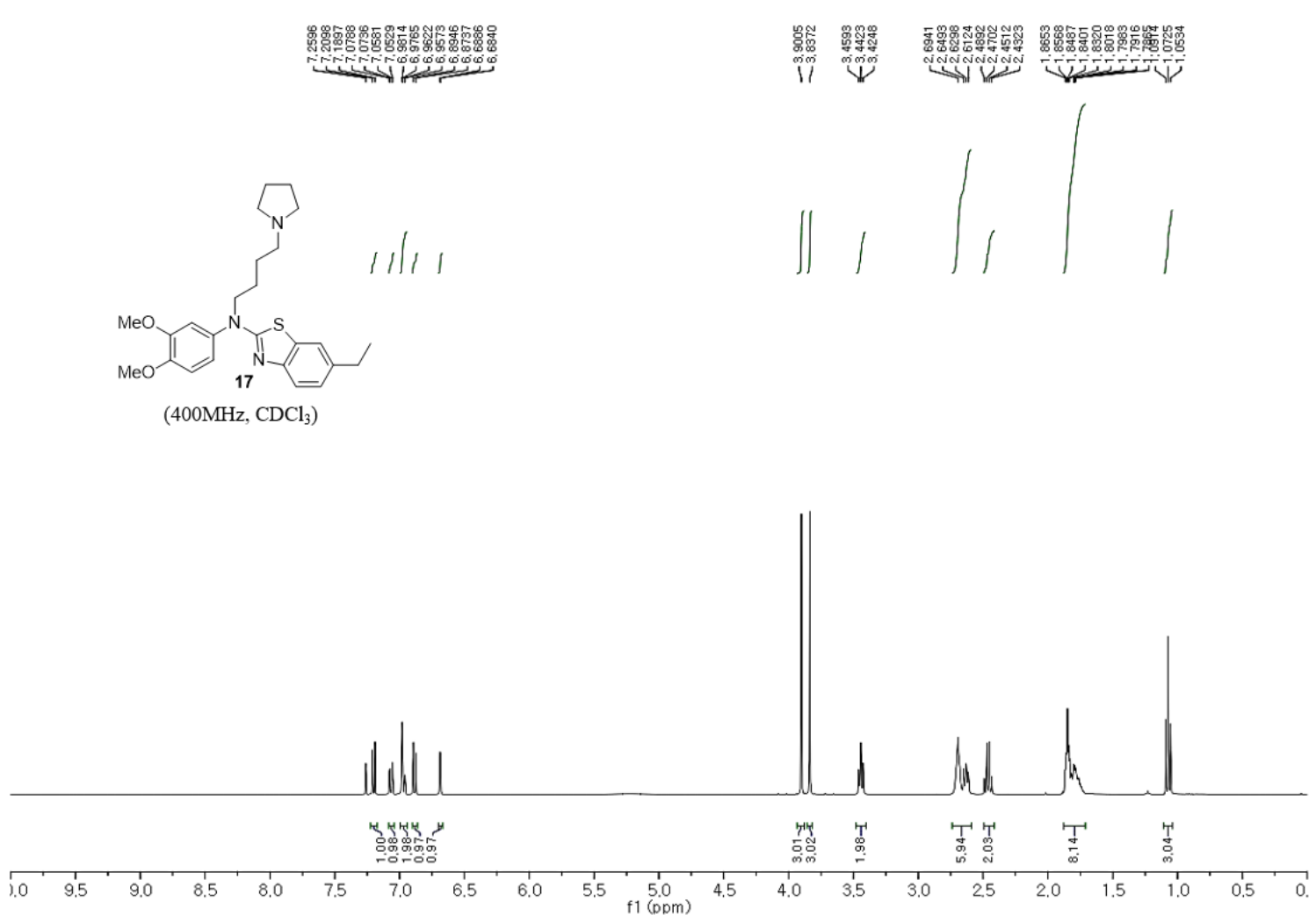

${ }^{1} \mathrm{H}$ NMR spectrum (400 MHz) of 17 in $\mathrm{CDCl}_{3}$

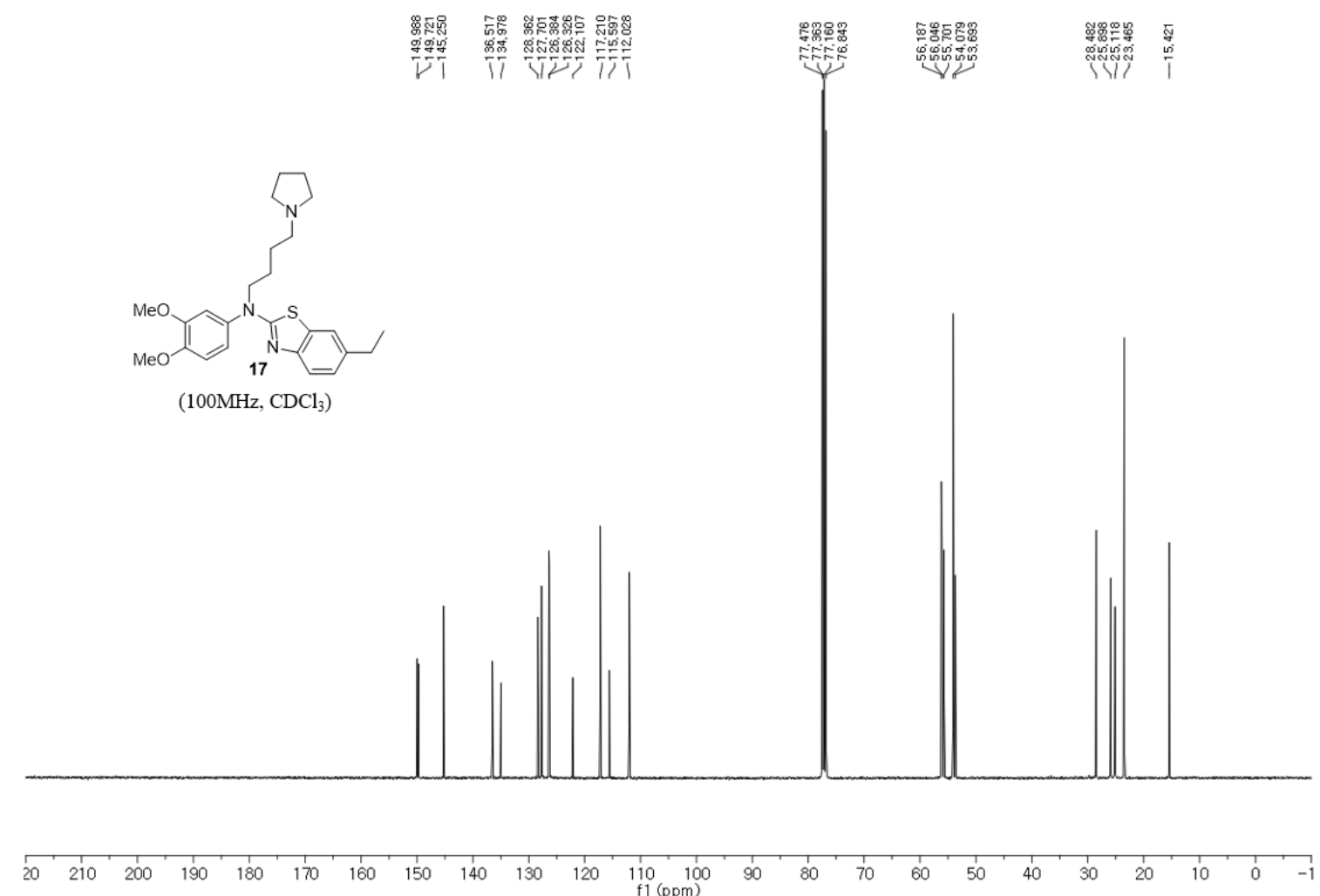

${ }^{13} \mathrm{C}$ NMR spectrum $(100 \mathrm{MHz})$ of $\mathbf{1 7}$ in $\mathrm{CDCl}_{3}$ 


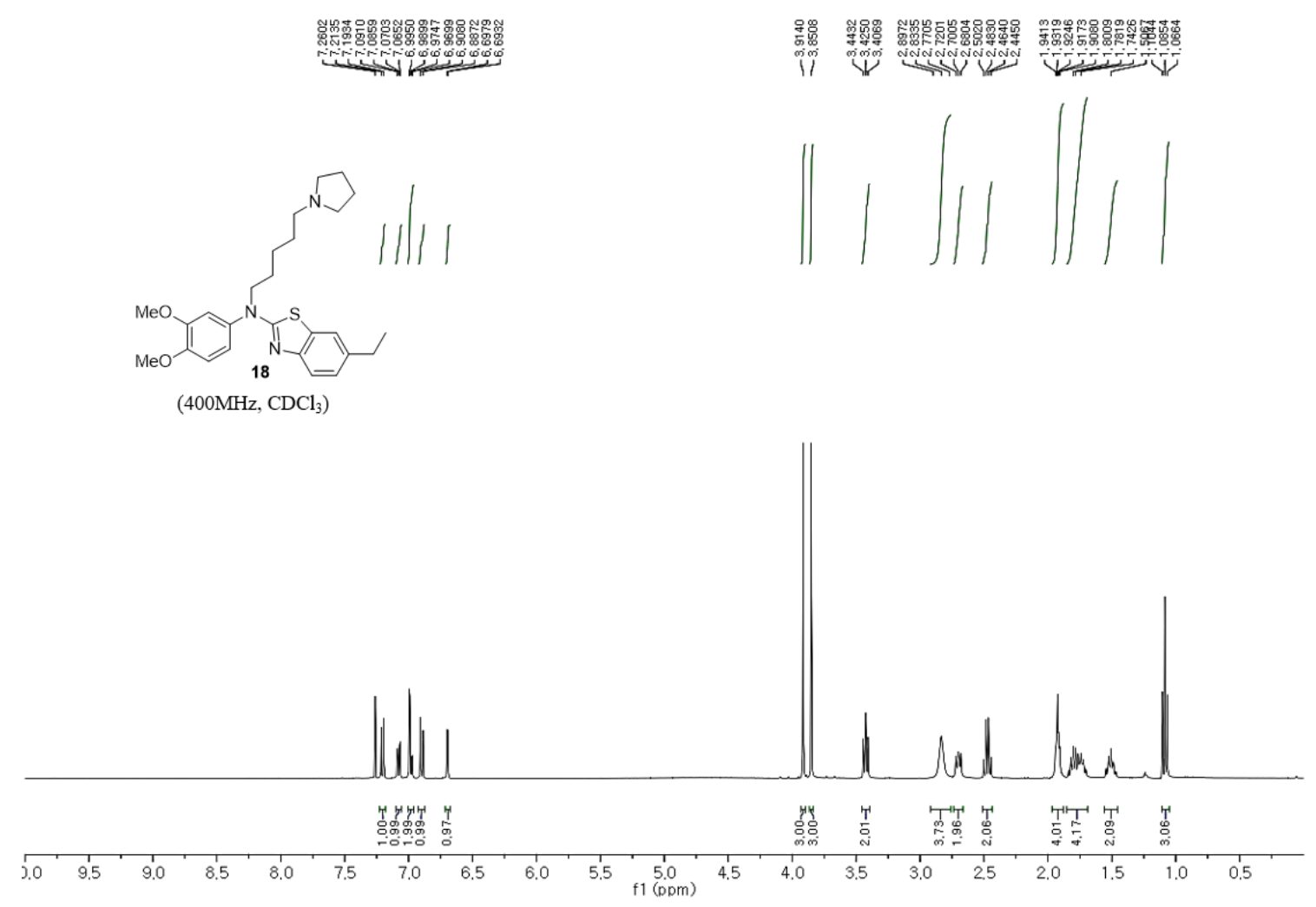

${ }^{1} \mathrm{H}$ NMR spectrum $(400 \mathrm{MHz})$ of $\mathbf{1 8}$ in $\mathrm{CDCl}_{3}$

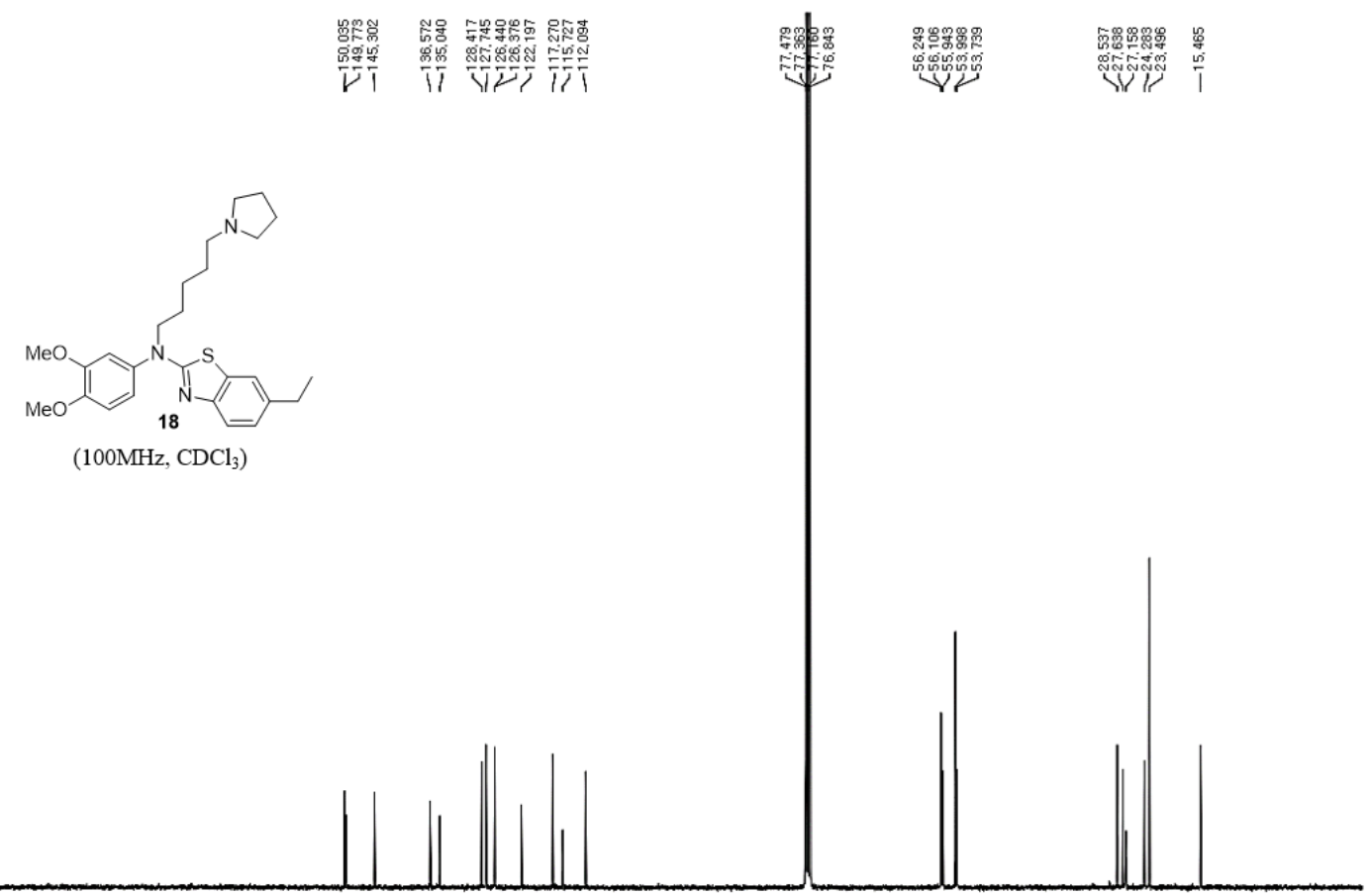

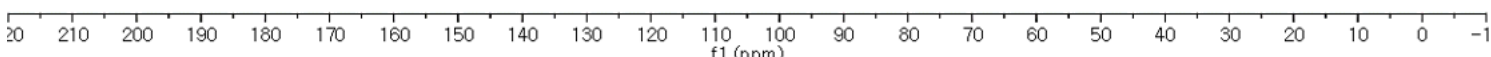

${ }^{13} \mathrm{C}$ NMR spectrum $(100 \mathrm{MHz})$ of $\mathbf{1 8}$ in $\mathrm{CDCl}_{3}$ 


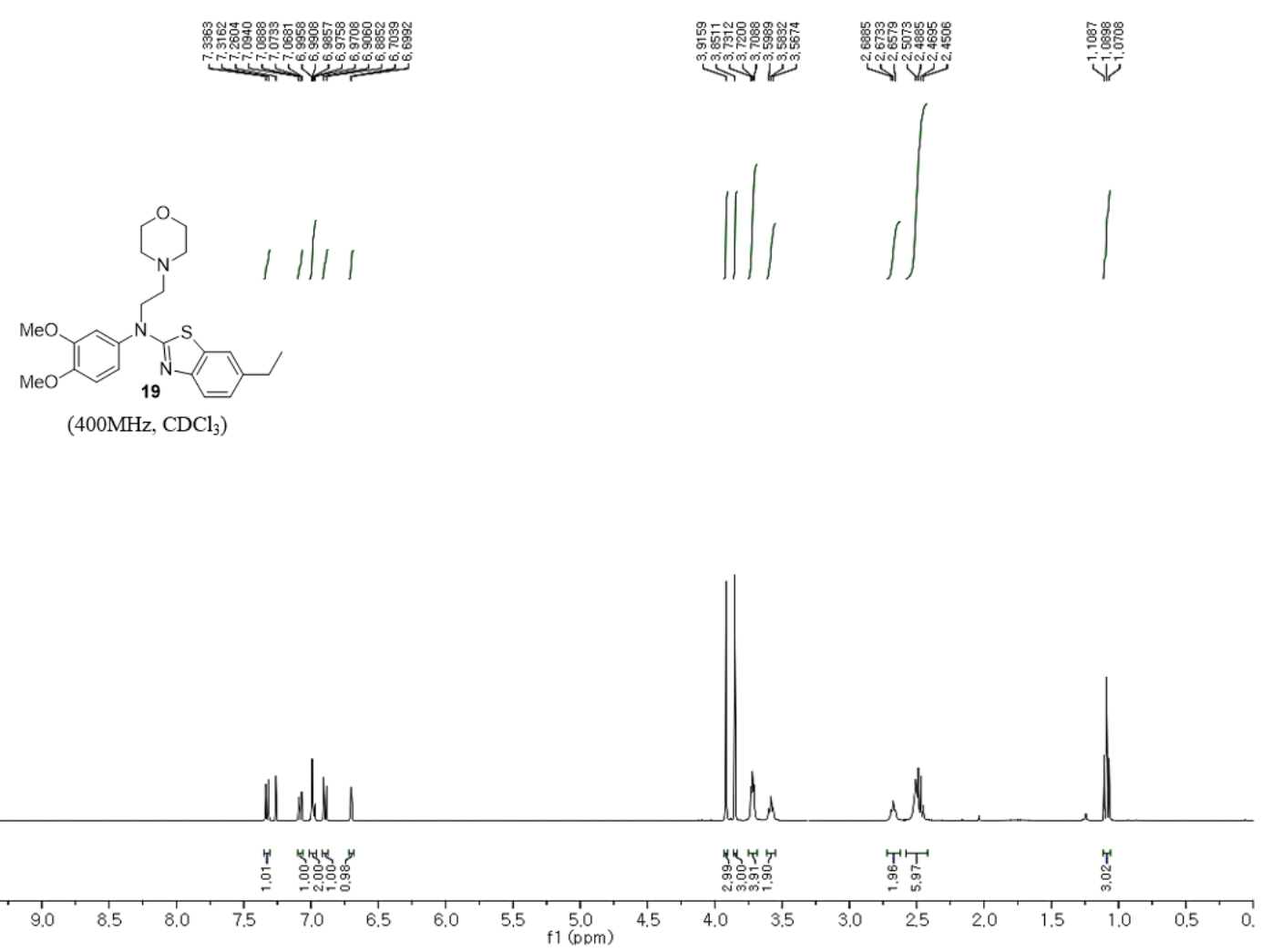

${ }^{1} \mathrm{H}$ NMR spectrum $\left(400 \mathrm{MHz}\right.$ ) of $\mathbf{1 9}$ in $\mathrm{CDCl}_{3}$

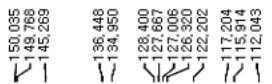

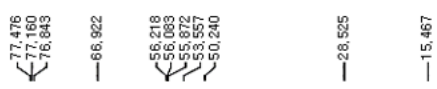

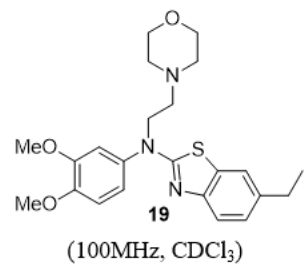

$\left(100 \mathrm{MHz}, \mathrm{CDCl}_{3}\right)$

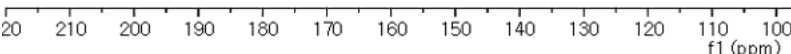

${ }^{13} \mathrm{C}$ NMR spectrum $(100 \mathrm{MHz})$ of $\mathbf{1 9}$ in $\mathrm{CDCl}_{3}$ 

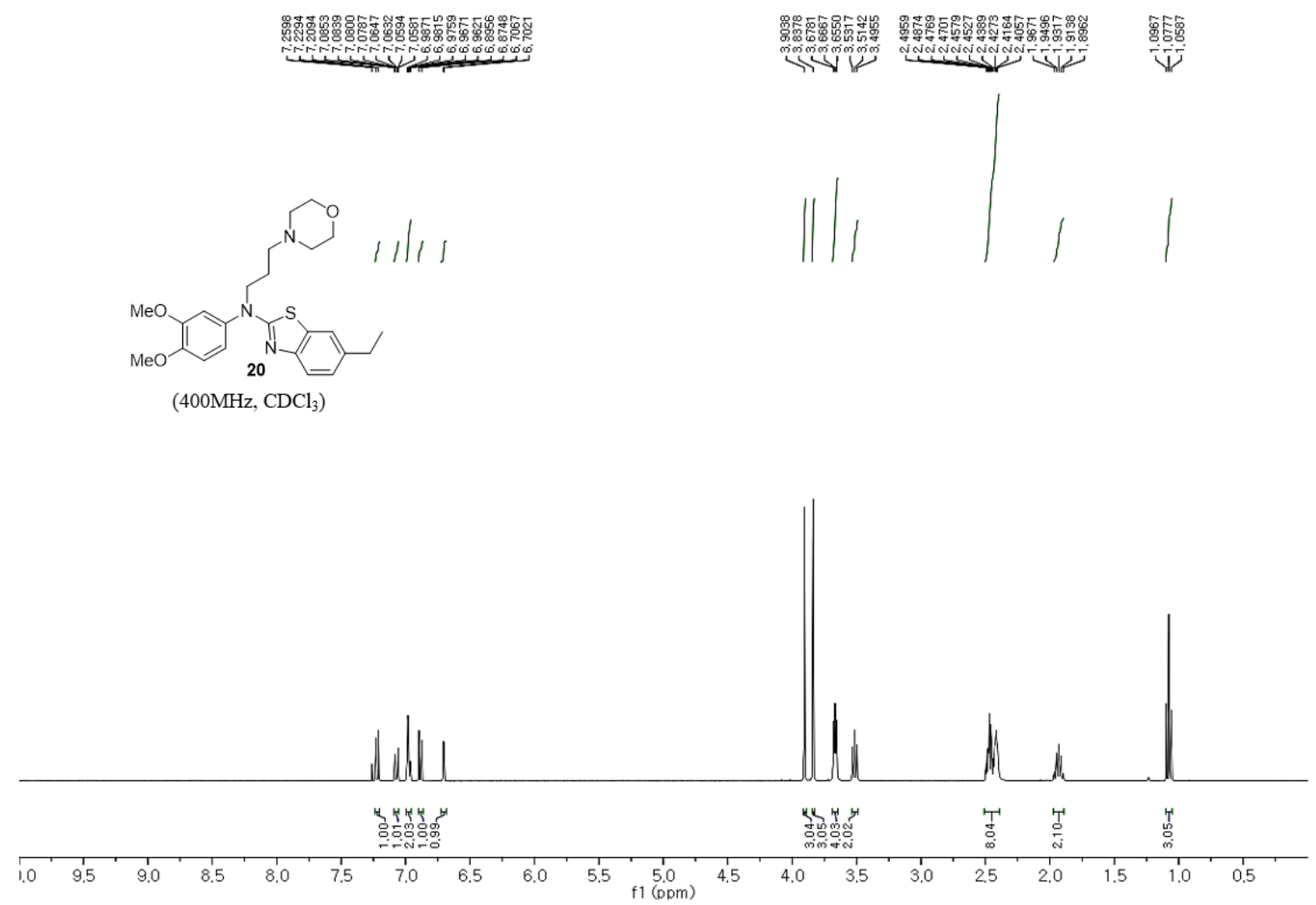

${ }^{1} \mathrm{H}$ NMR spectrum (400 MHz) of 20 in $\mathrm{CDCl}_{3}$

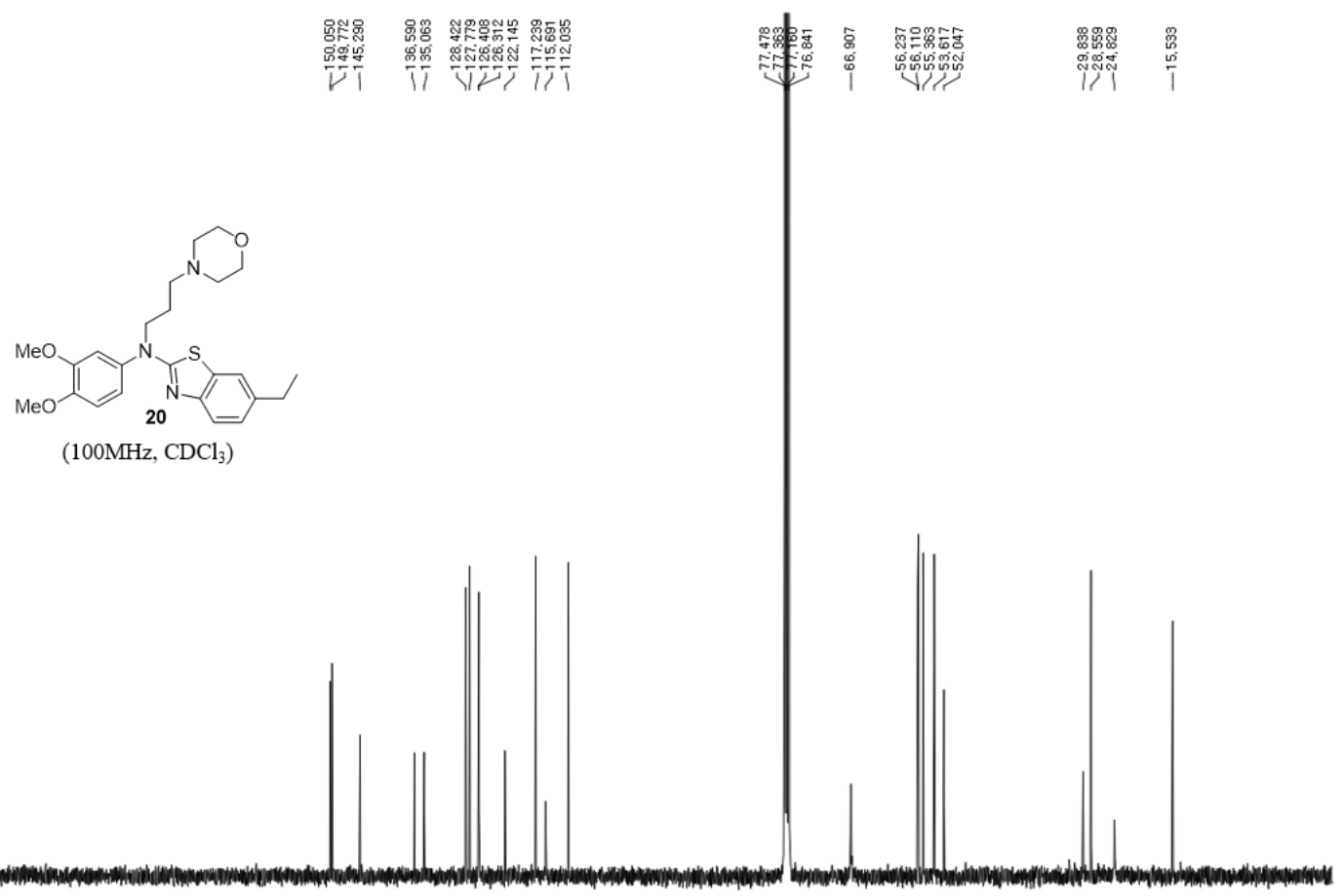

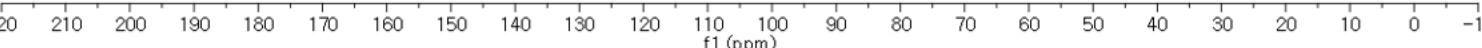

${ }^{13} \mathrm{C}$ NMR spectrum $(100 \mathrm{MHz})$ of $\mathbf{2 0}$ in $\mathrm{CDCl}_{3}$ 

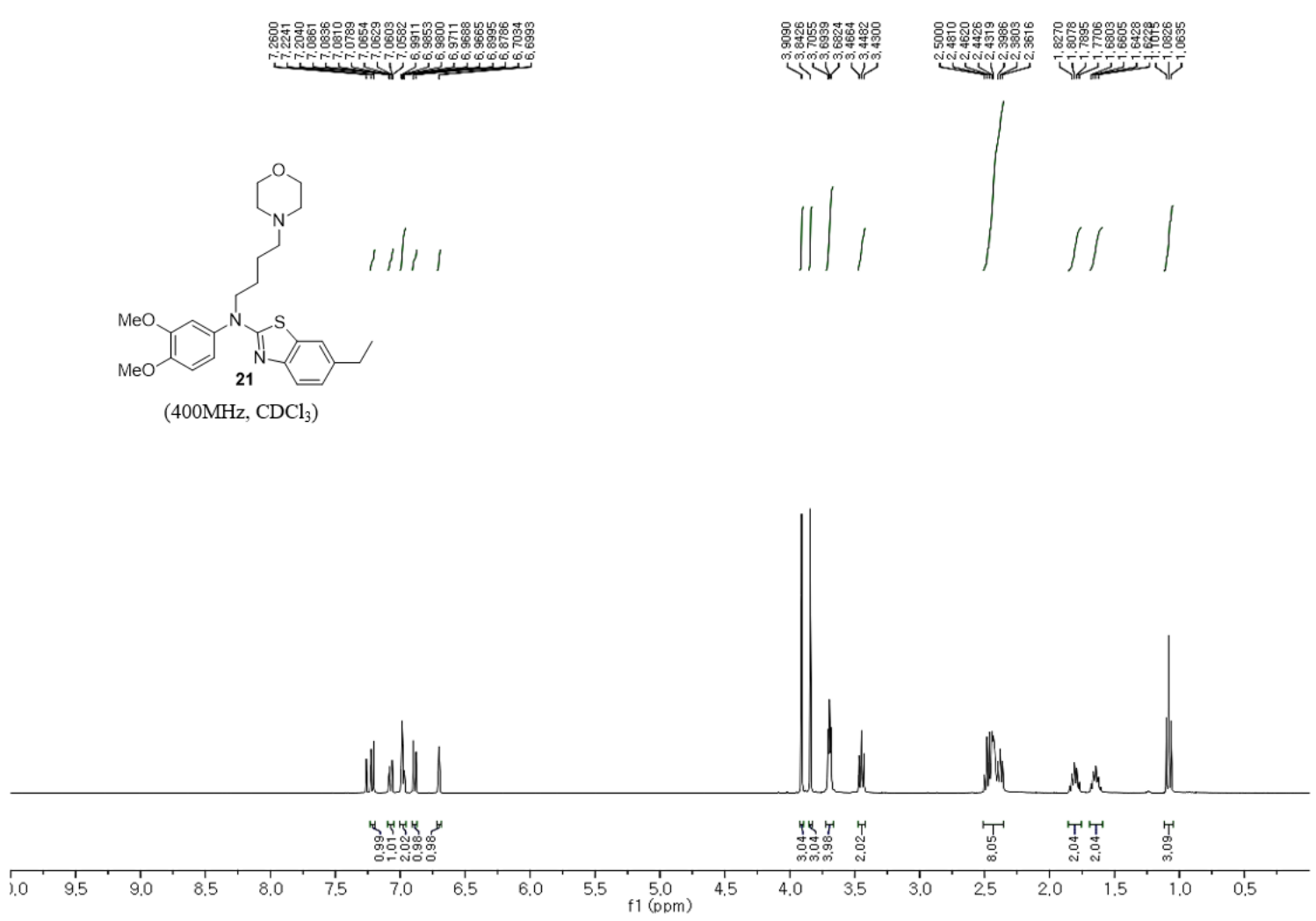

${ }^{1} \mathrm{H}$ NMR spectrum $(400 \mathrm{MHz})$ of 21 in $\mathrm{CDCl}_{3}$

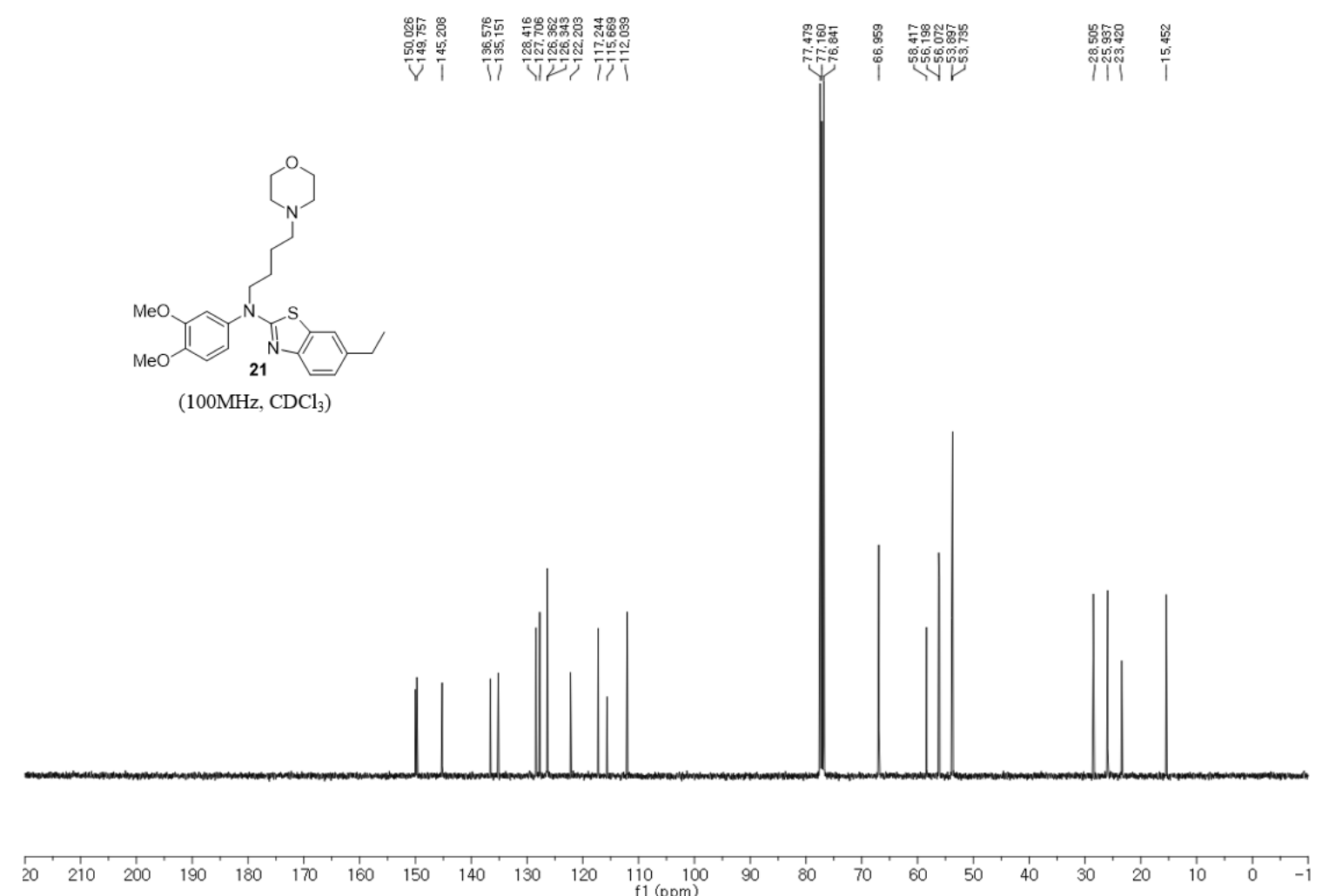

${ }^{13} \mathrm{C}$ NMR spectrum $(100 \mathrm{MHz})$ of 21 in $\mathrm{CDCl}_{3}$ 

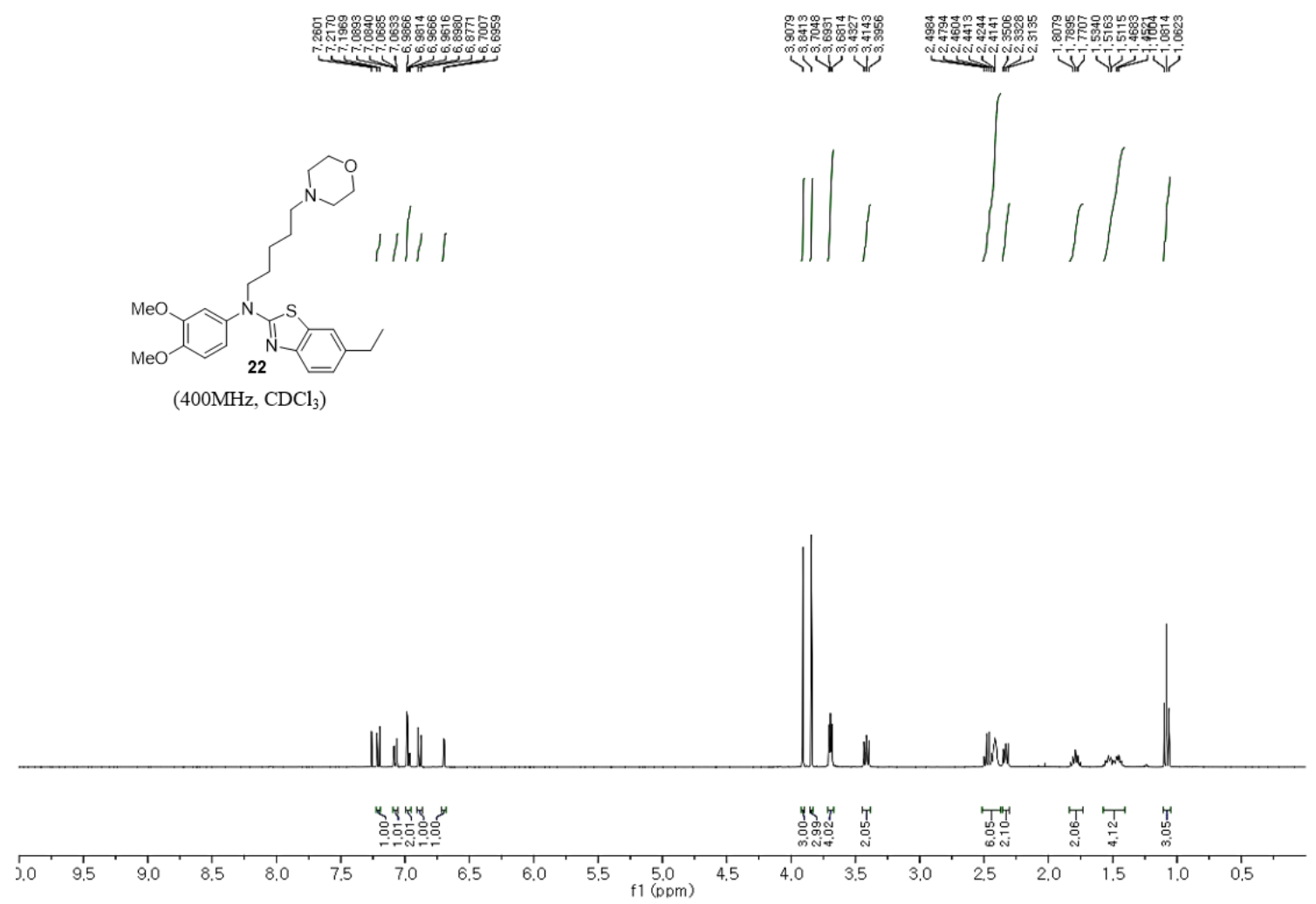

${ }^{1} \mathrm{H}$ NMR spectrum (400 MHz) of 22 in $\mathrm{CDCl}_{3}$
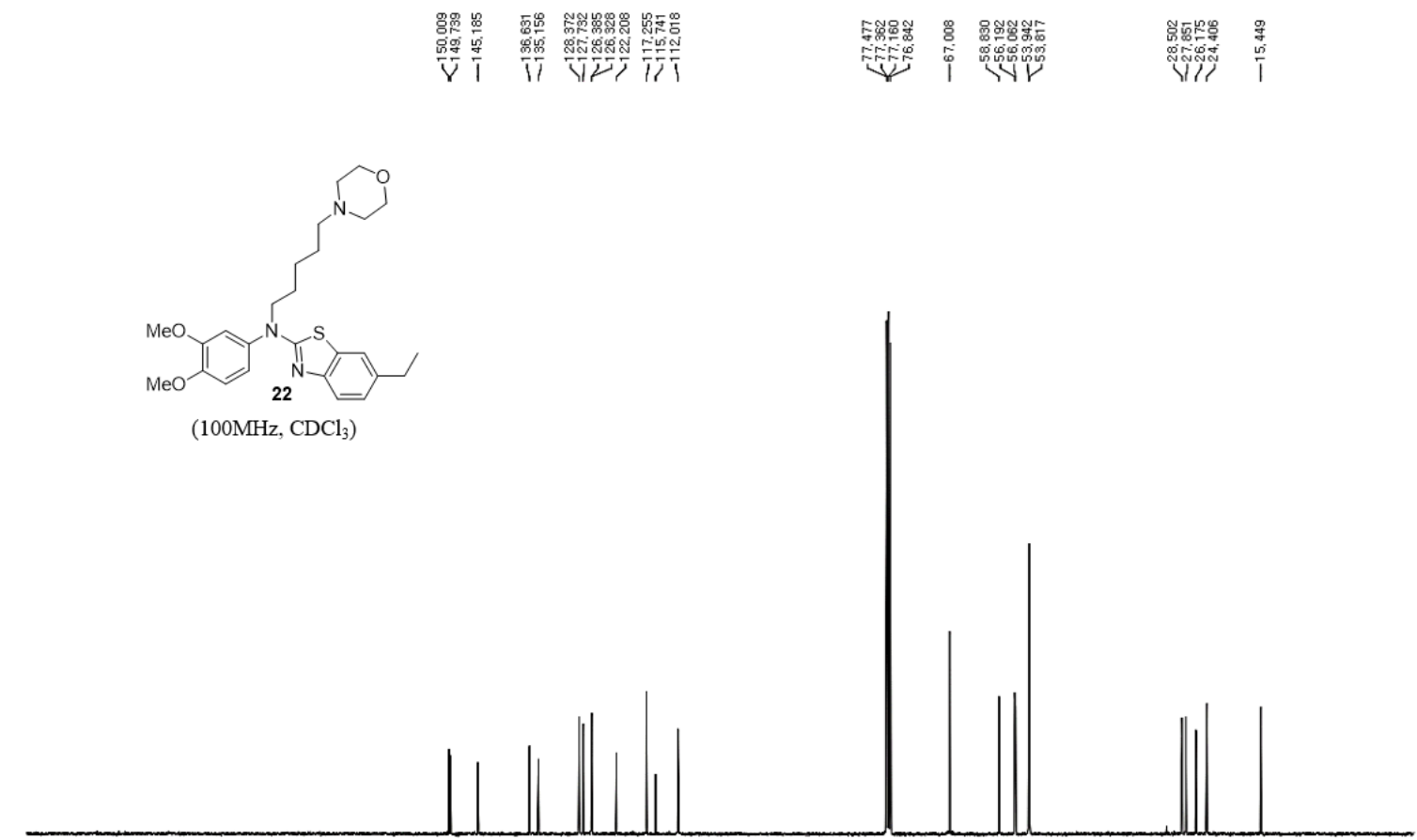

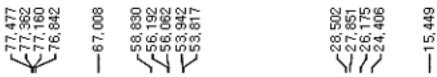

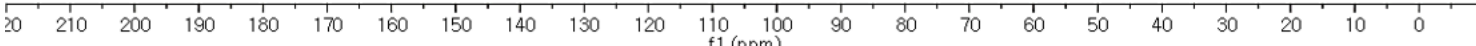

${ }^{13} \mathrm{C}$ NMR spectrum (100 MHz) of 22 in $\mathrm{CDCl}_{3}$ 

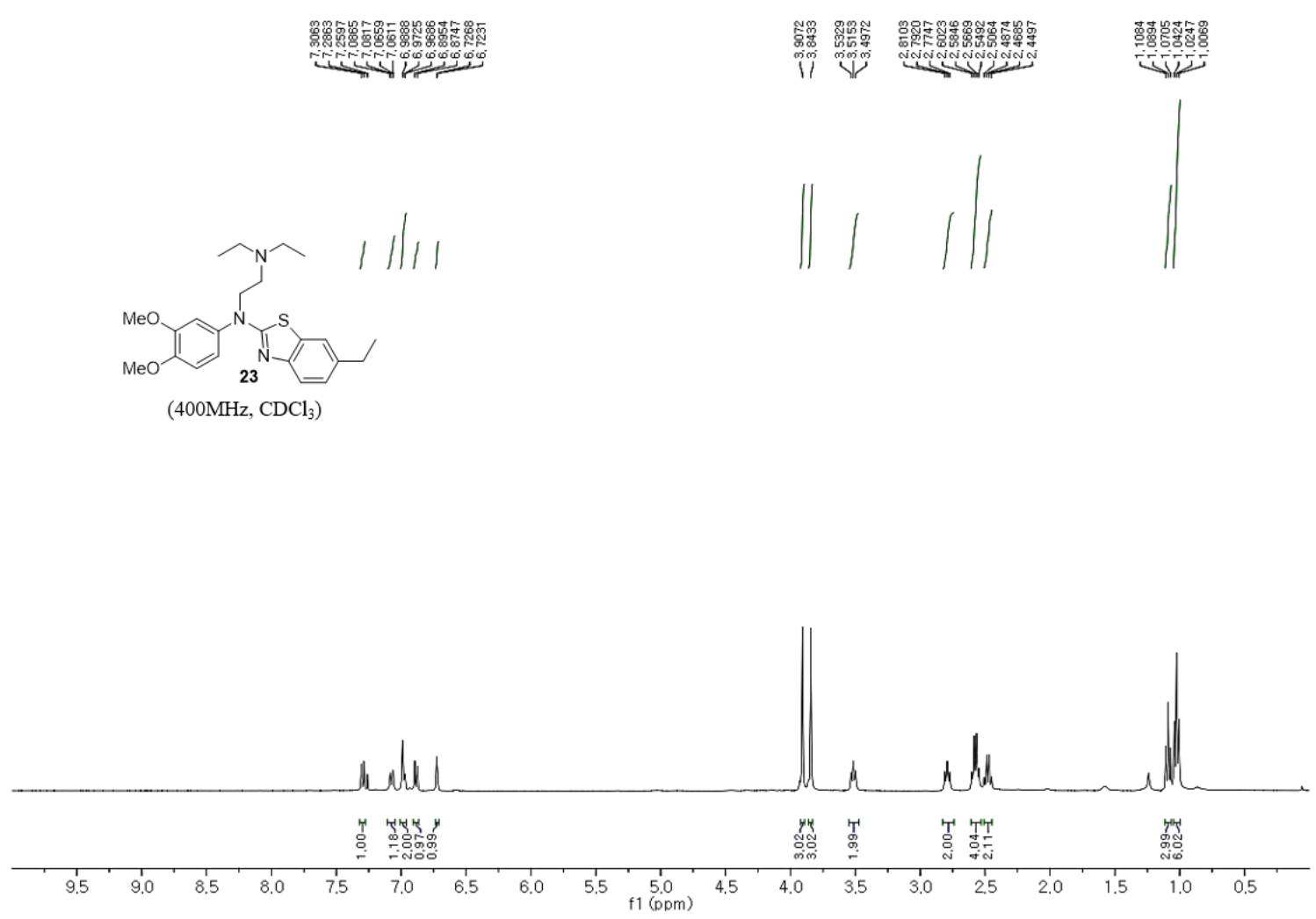

${ }^{1} \mathrm{H}$ NMR spectrum $(400 \mathrm{MHz})$ of $\mathbf{2 3}$ in $\mathrm{CDCl}_{3}$

\begin{tabular}{|c|c|c|c|}
\hline 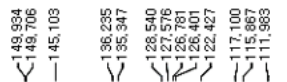 & 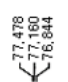 & 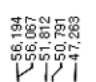 & 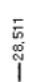 \\
\hline
\end{tabular}
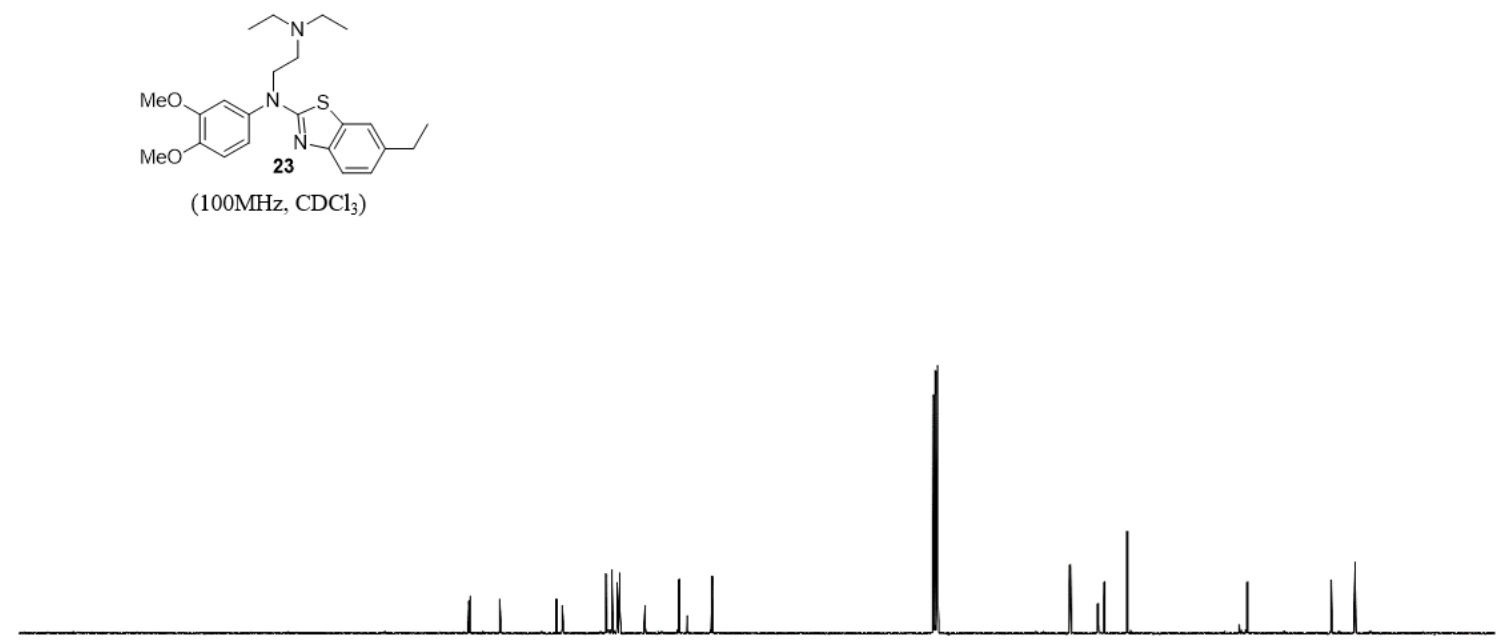

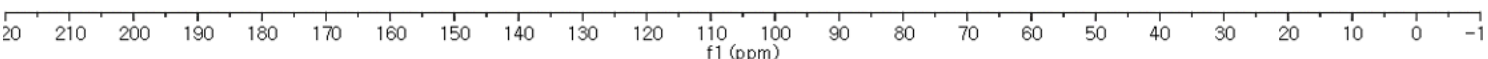

${ }^{13} \mathrm{C}$ NMR spectrum $(100 \mathrm{MHz})$ of 23 in $\mathrm{CDCl}_{3}$ 

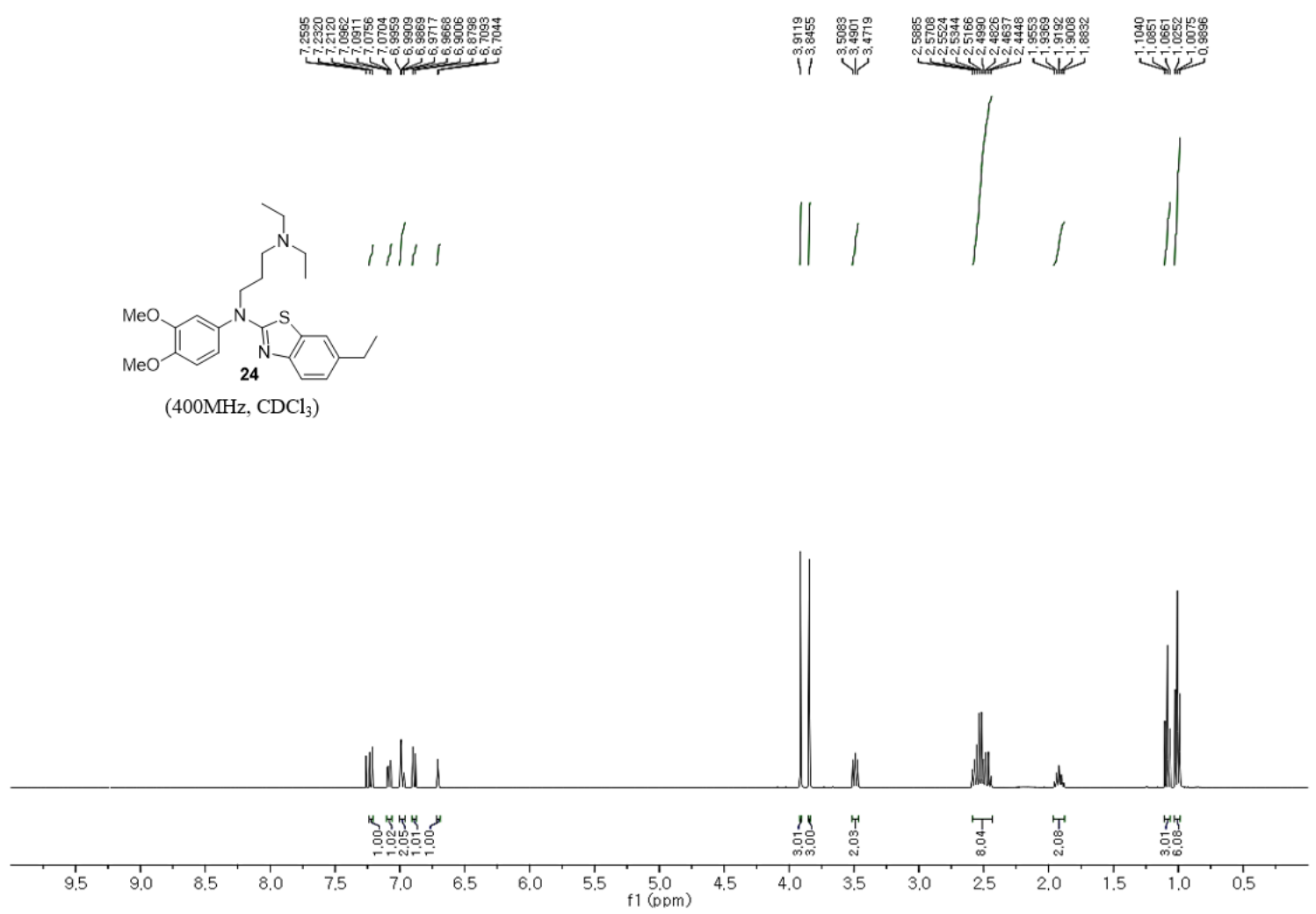

${ }^{1} \mathrm{H}$ NMR spectrum $(400 \mathrm{MHz})$ of 24 in $\mathrm{CDCl}_{3}$

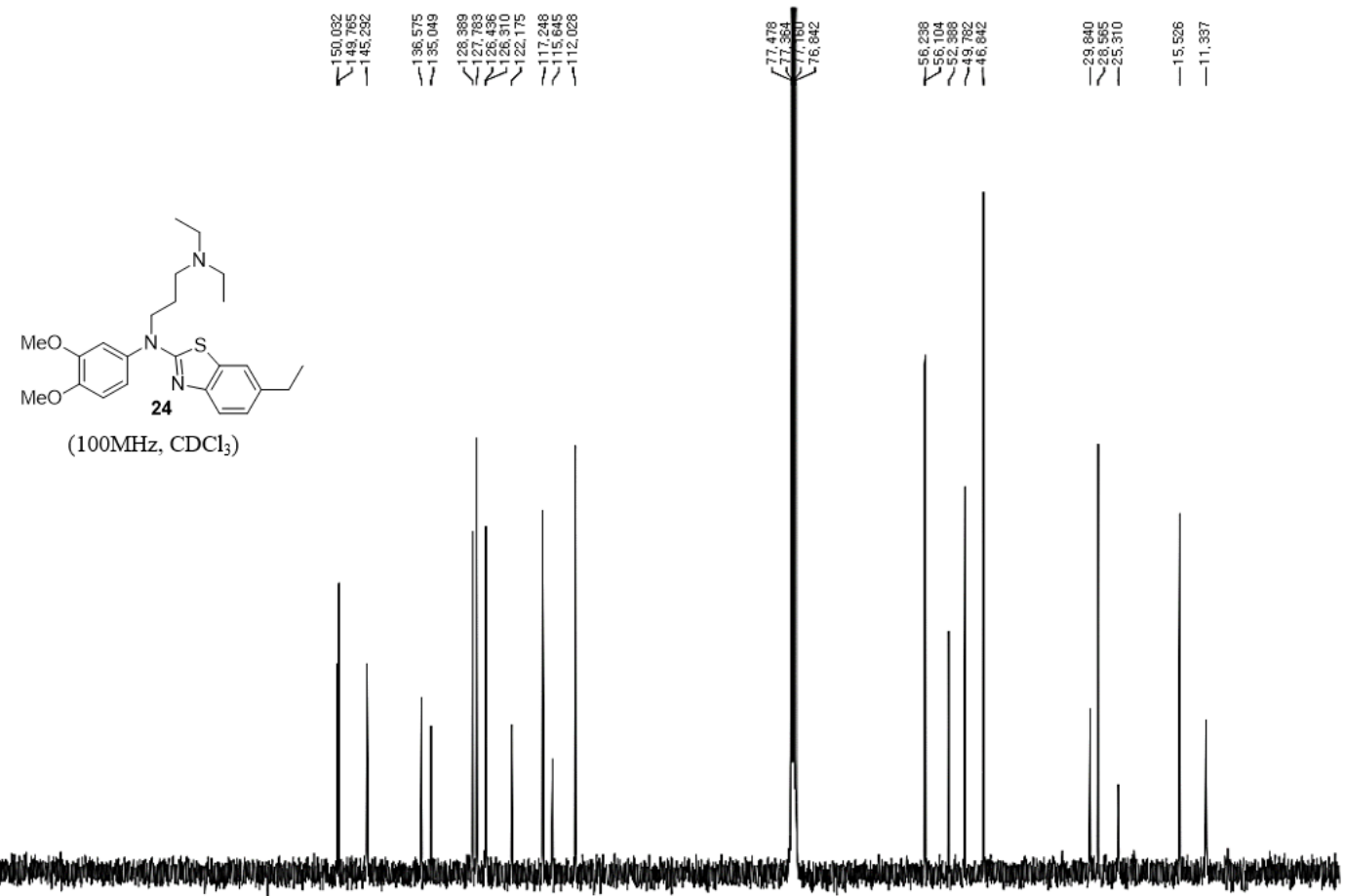

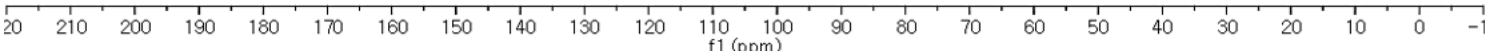

${ }^{13} \mathrm{C}$ NMR spectrum $(100 \mathrm{MHz})$ of 24 in $\mathrm{CDCl}_{3}$ 

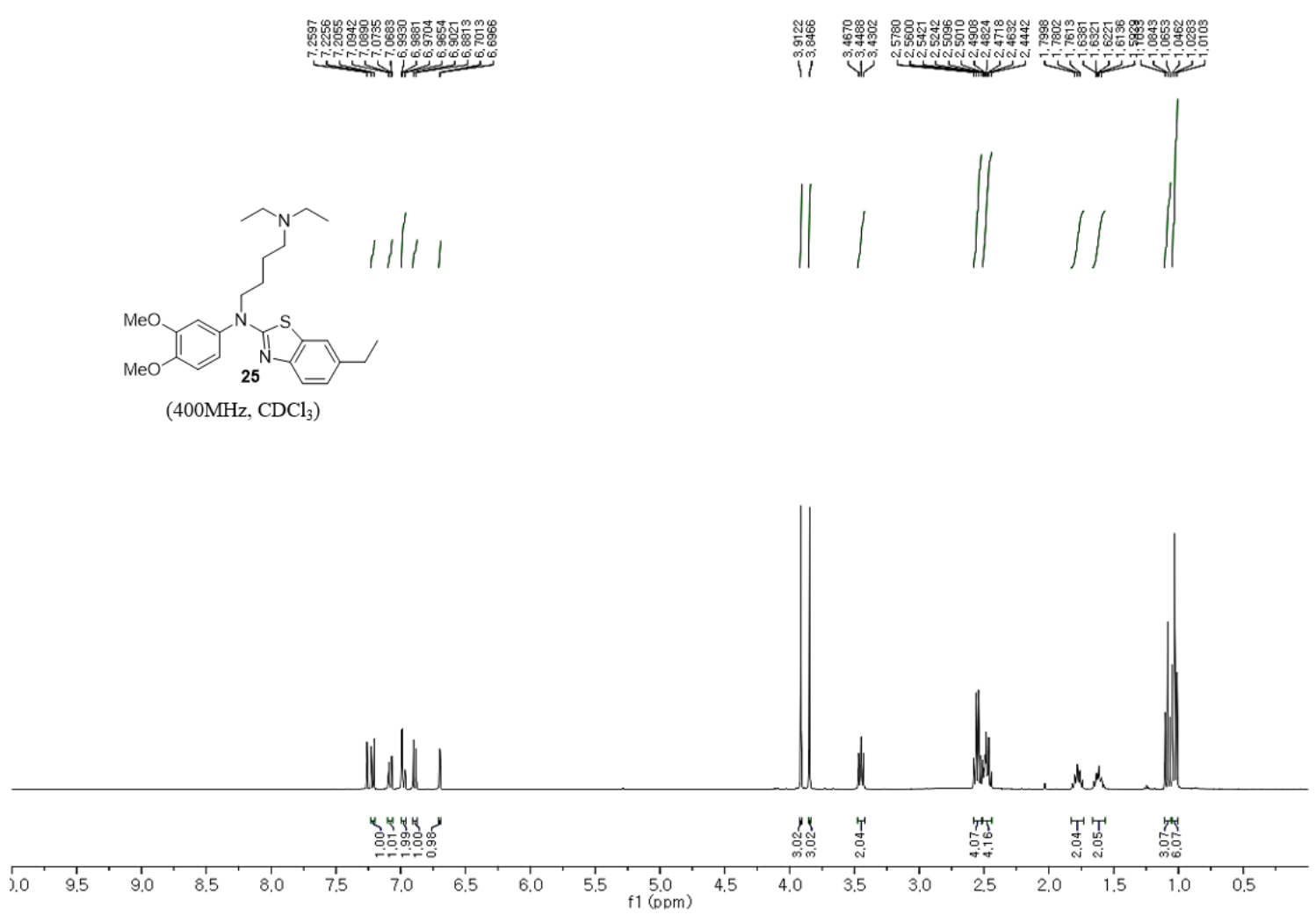

${ }^{1} \mathrm{H}$ NMR spectrum $(400 \mathrm{MHz})$ of 25 in $\mathrm{CDCl}_{3}$

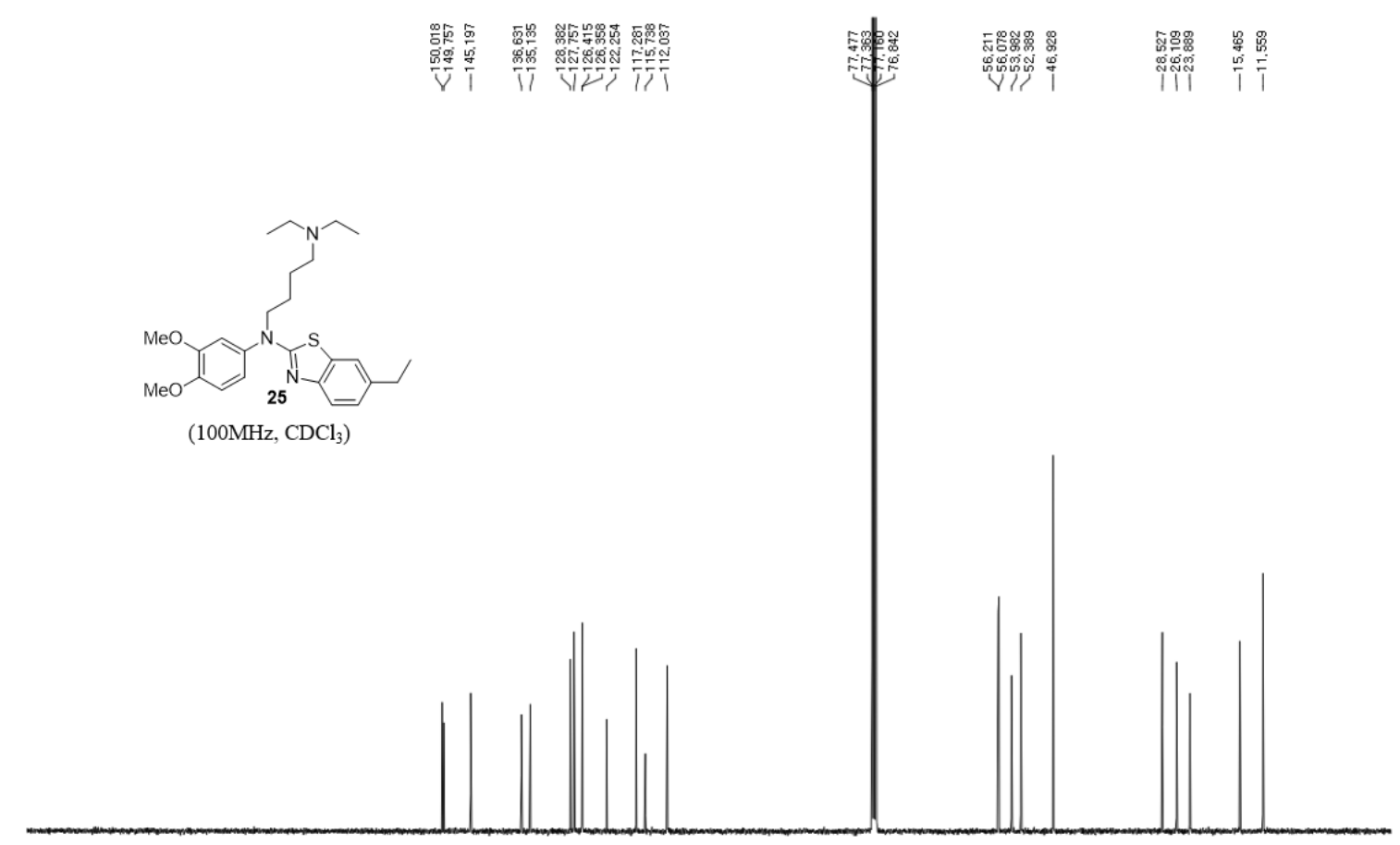

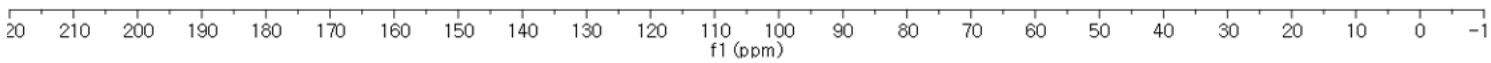

${ }^{13} \mathrm{C}$ NMR spectrum (100 MHz) of 25 in $\mathrm{CDCl}_{3}$ 

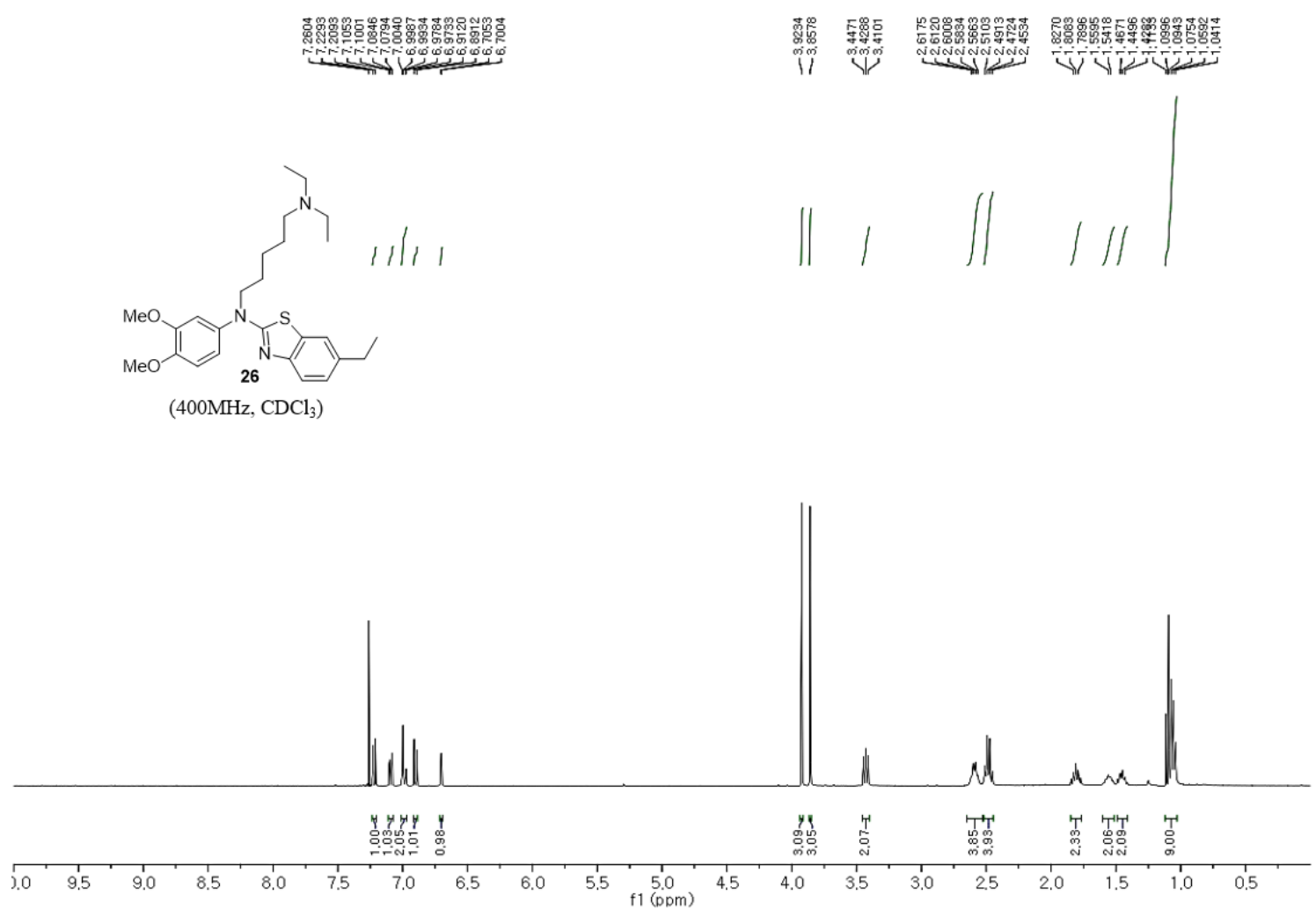

${ }^{1} \mathrm{H}$ NMR spectrum $(400 \mathrm{MHz})$ of $\mathbf{2 6}$ in $\mathrm{CDCl}_{3}$

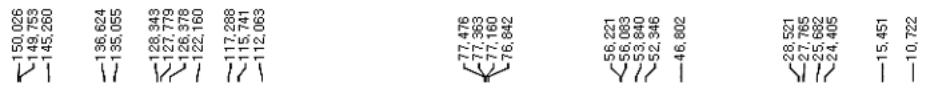

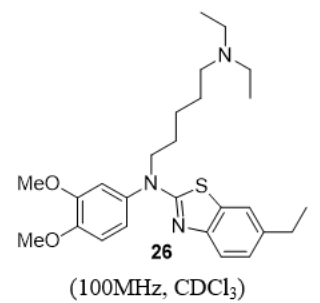

$\left(100 \mathrm{MHz}, \mathrm{CDCl}_{3}\right)$

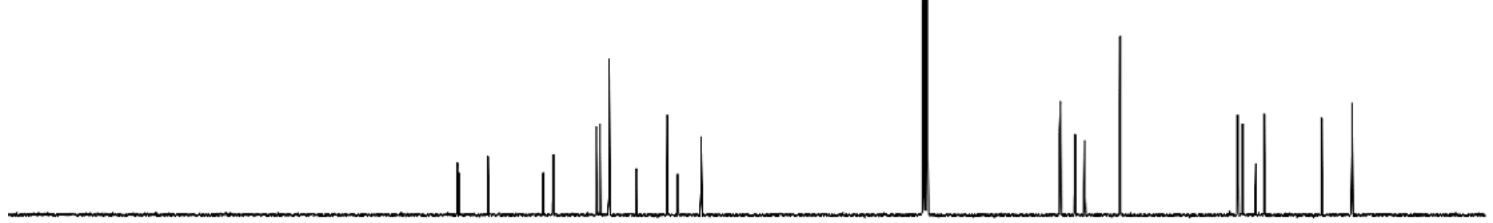

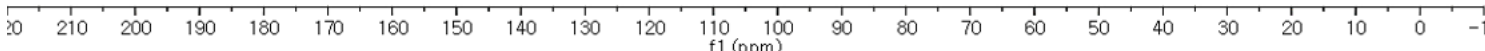

${ }^{13} \mathrm{C}$ NMR spectrum $(100 \mathrm{MHz})$ of 26 in $\mathrm{CDCl}_{3}$ 

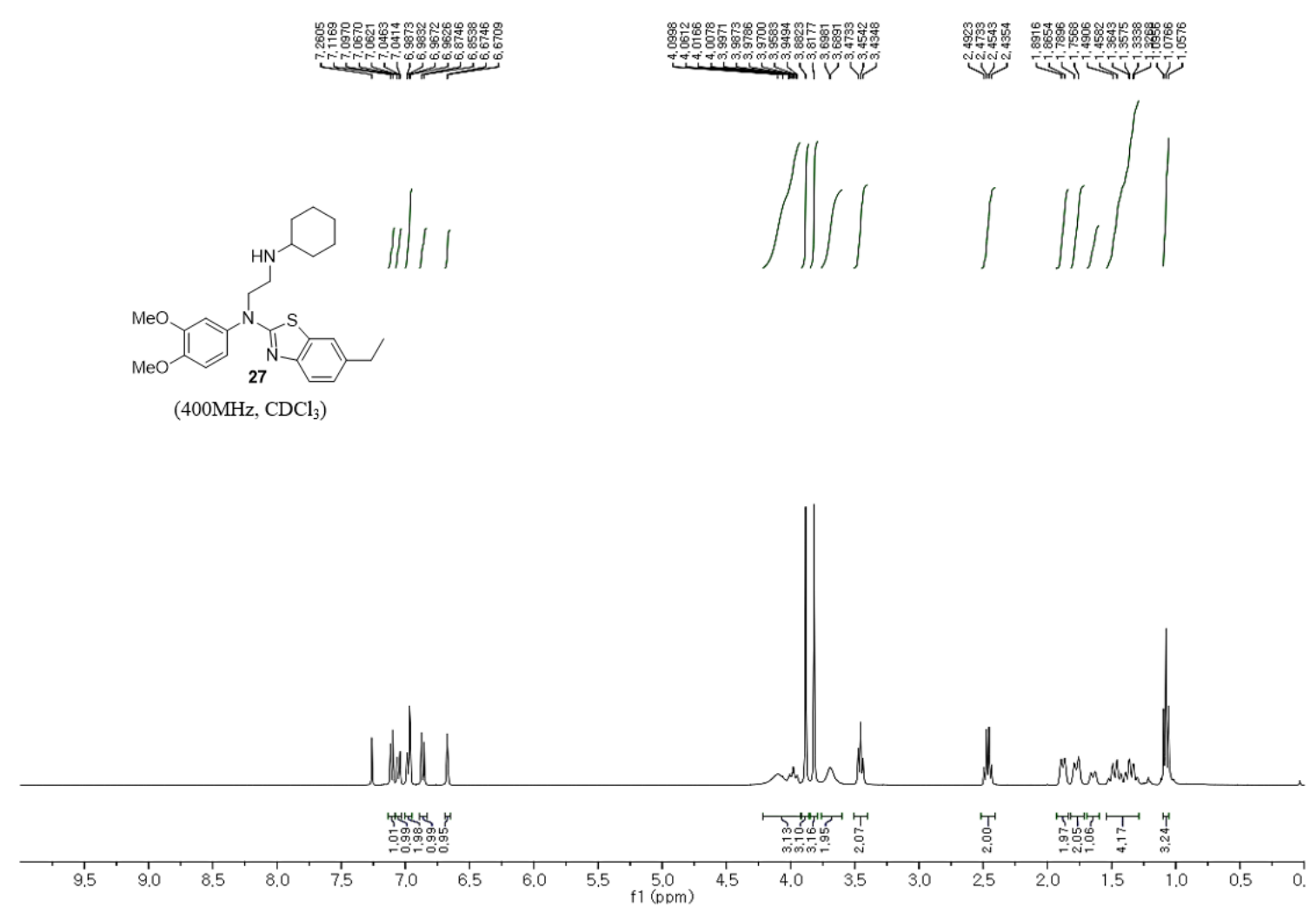

${ }^{1} \mathrm{H}$ NMR spectrum (400 MHz) of 27 in $\mathrm{CDCl}_{3}$

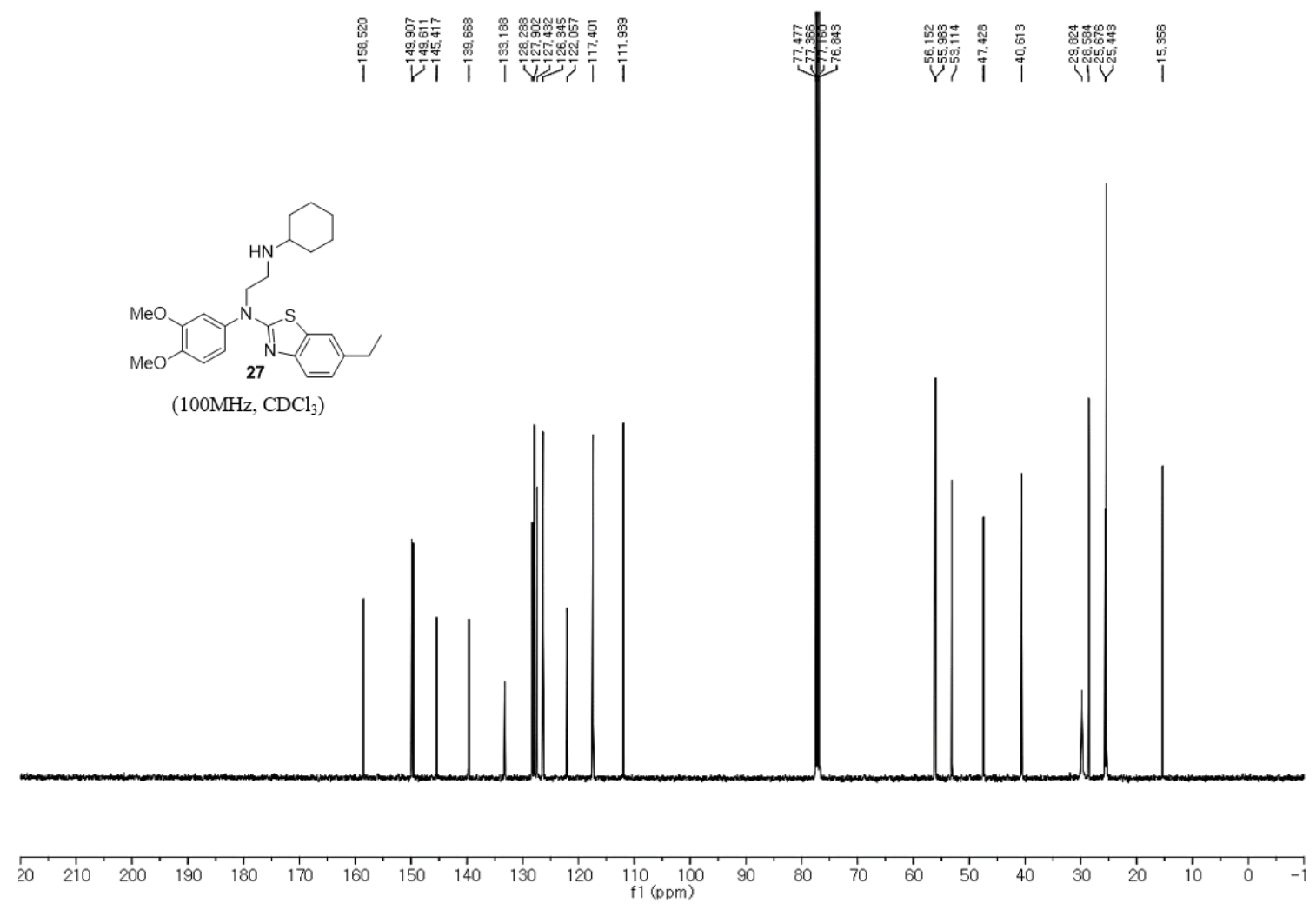

${ }^{13} \mathrm{C}$ NMR spectrum $(100 \mathrm{MHz})$ of 27 in $\mathrm{CDCl}_{3}$ 


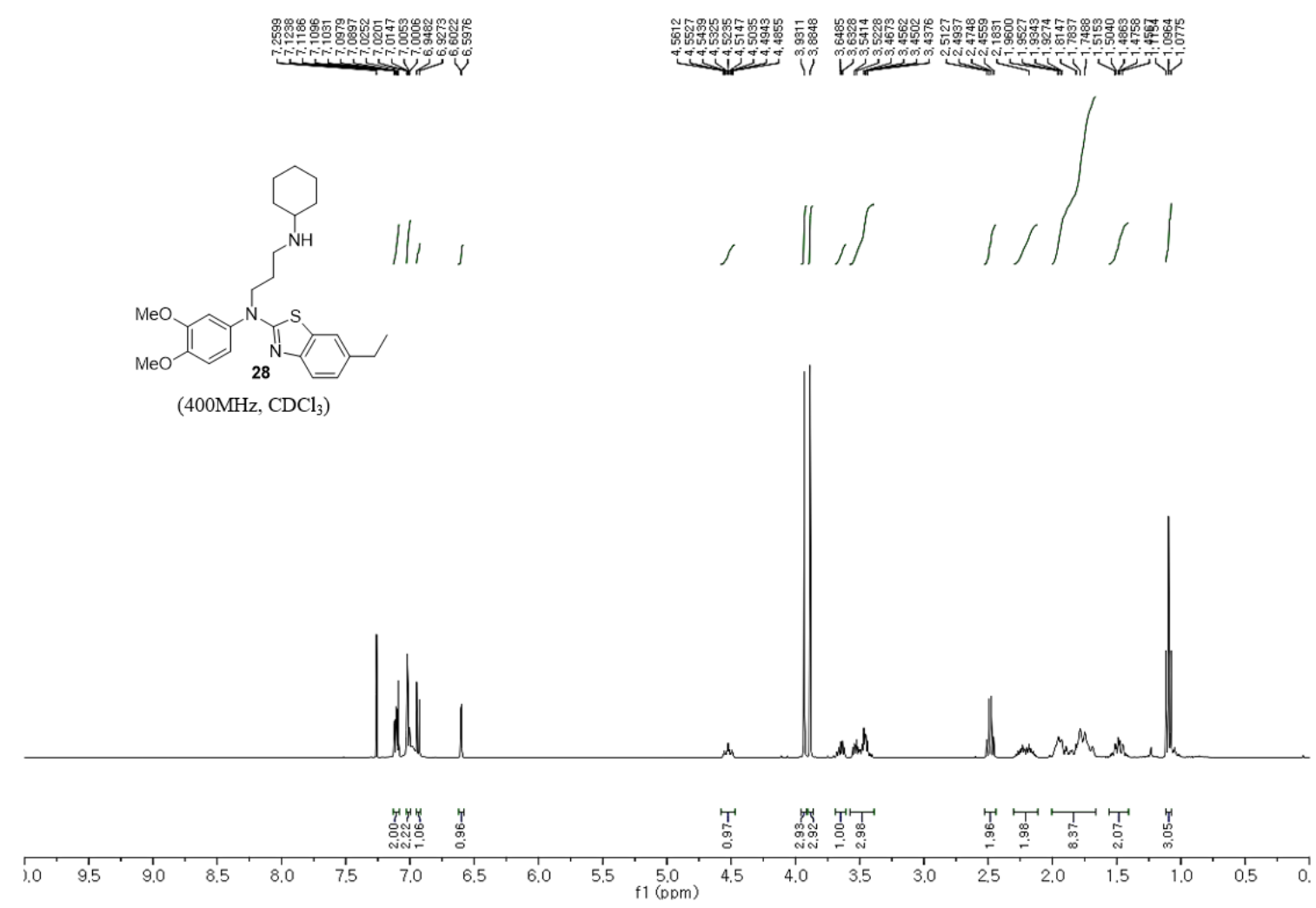

${ }^{1} \mathrm{H}$ NMR spectrum (400 MHz) of $\mathbf{2 8}$ in $\mathrm{CDCl}_{3}$

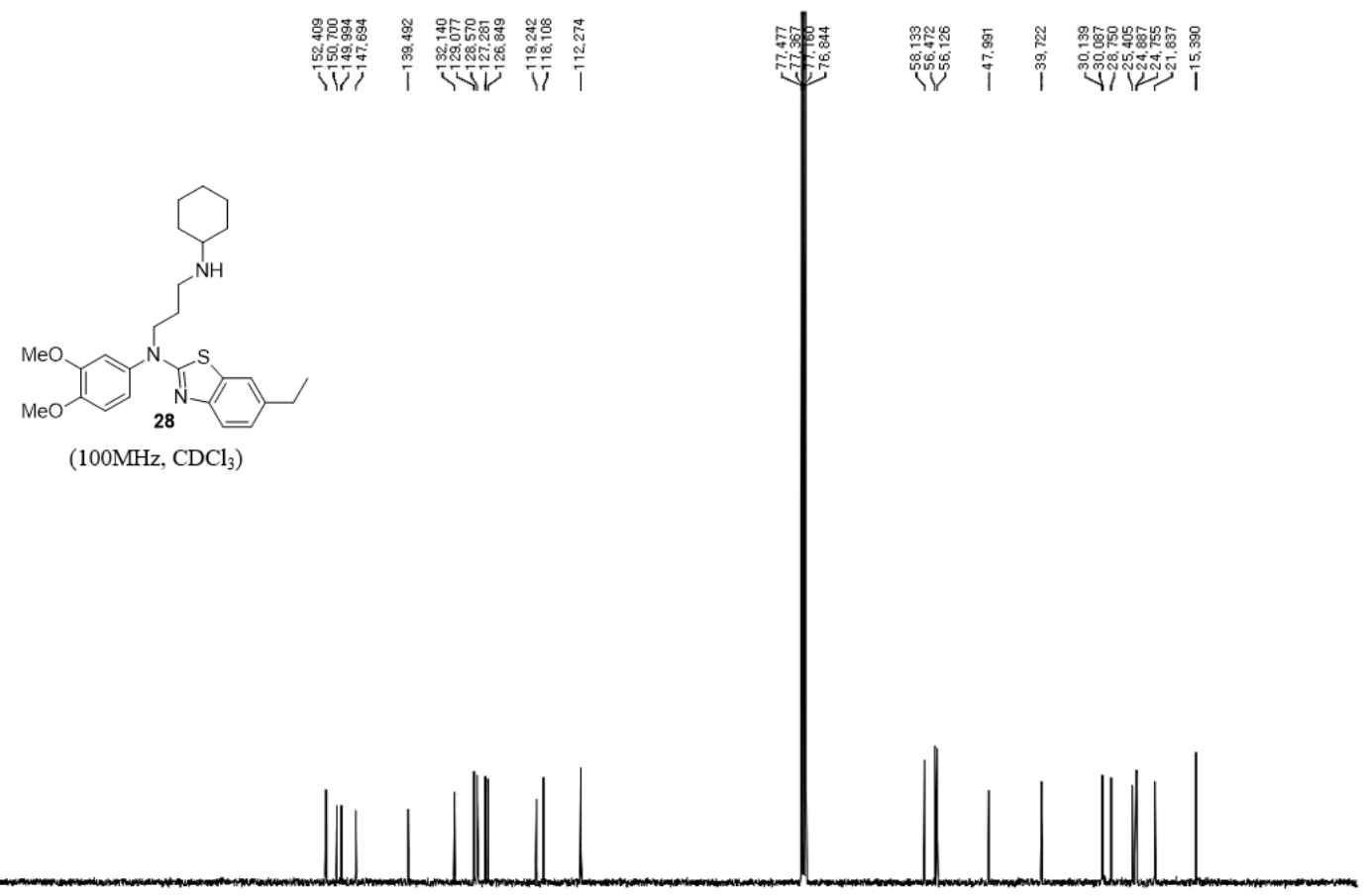

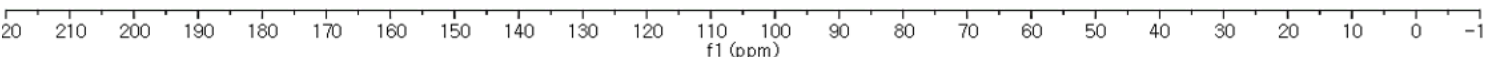

${ }^{13} \mathrm{C}$ NMR spectrum $(100 \mathrm{MHz})$ of 28 in $\mathrm{CDCl}_{3}$ 

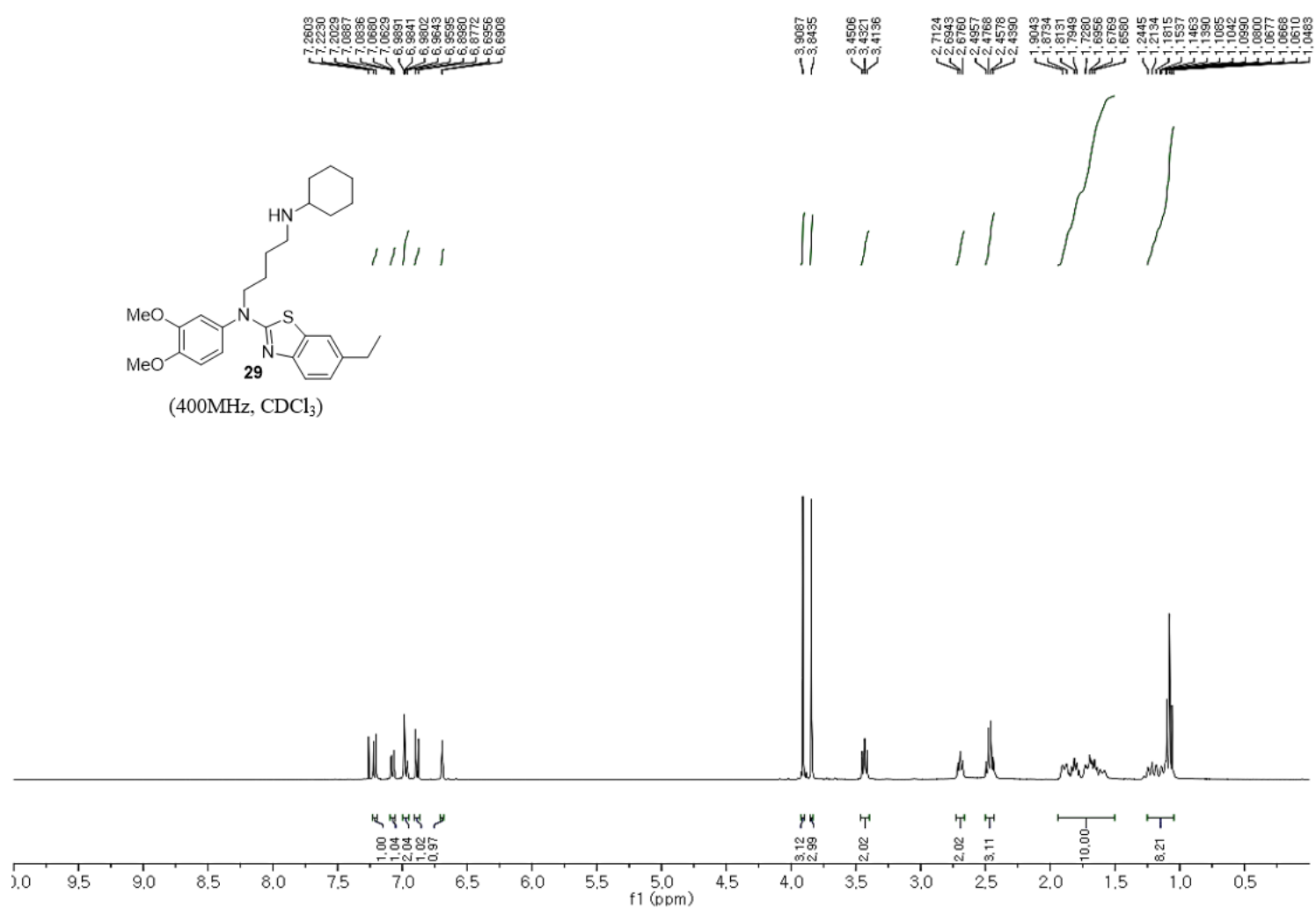

${ }^{1} \mathrm{H}$ NMR spectrum (400 MHz) of 29 in $\mathrm{CDCl}_{3}$

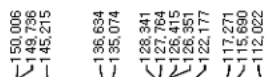

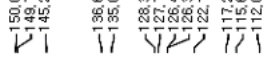

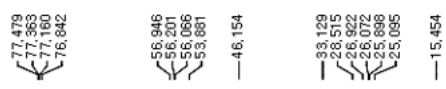

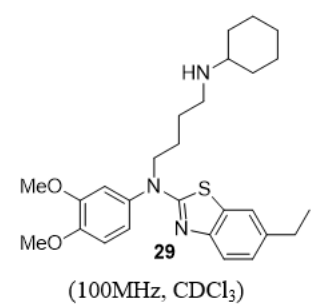

$\left(100 \mathrm{MHz}, \mathrm{CDCl}_{3}\right)$

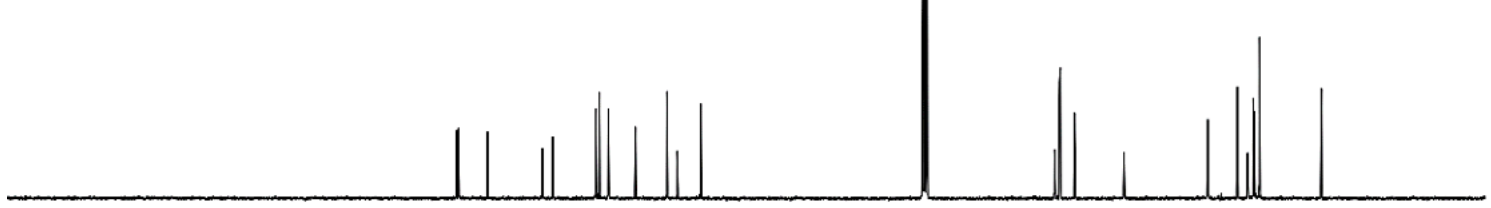

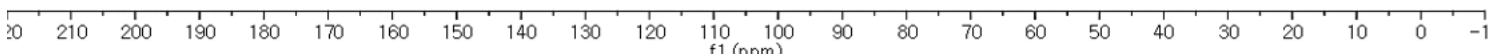

${ }^{13} \mathrm{C}$ NMR spectrum $(100 \mathrm{MHz})$ of 29 in $\mathrm{CDCl}_{3}$ 


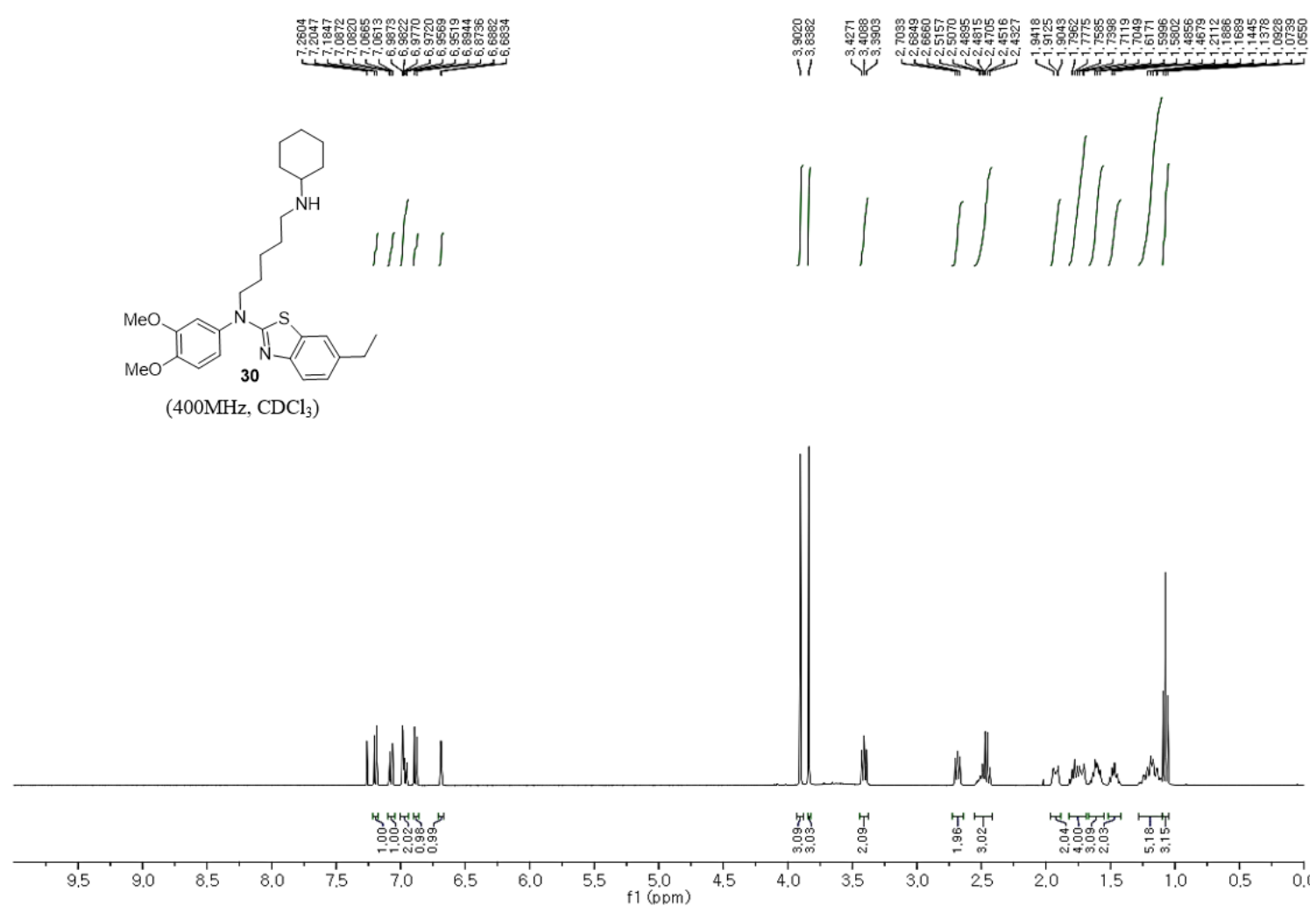

${ }^{1} \mathrm{H}$ NMR spectrum (400 MHz) of $\mathbf{3 0}$ in $\mathrm{CDCl}_{3}$

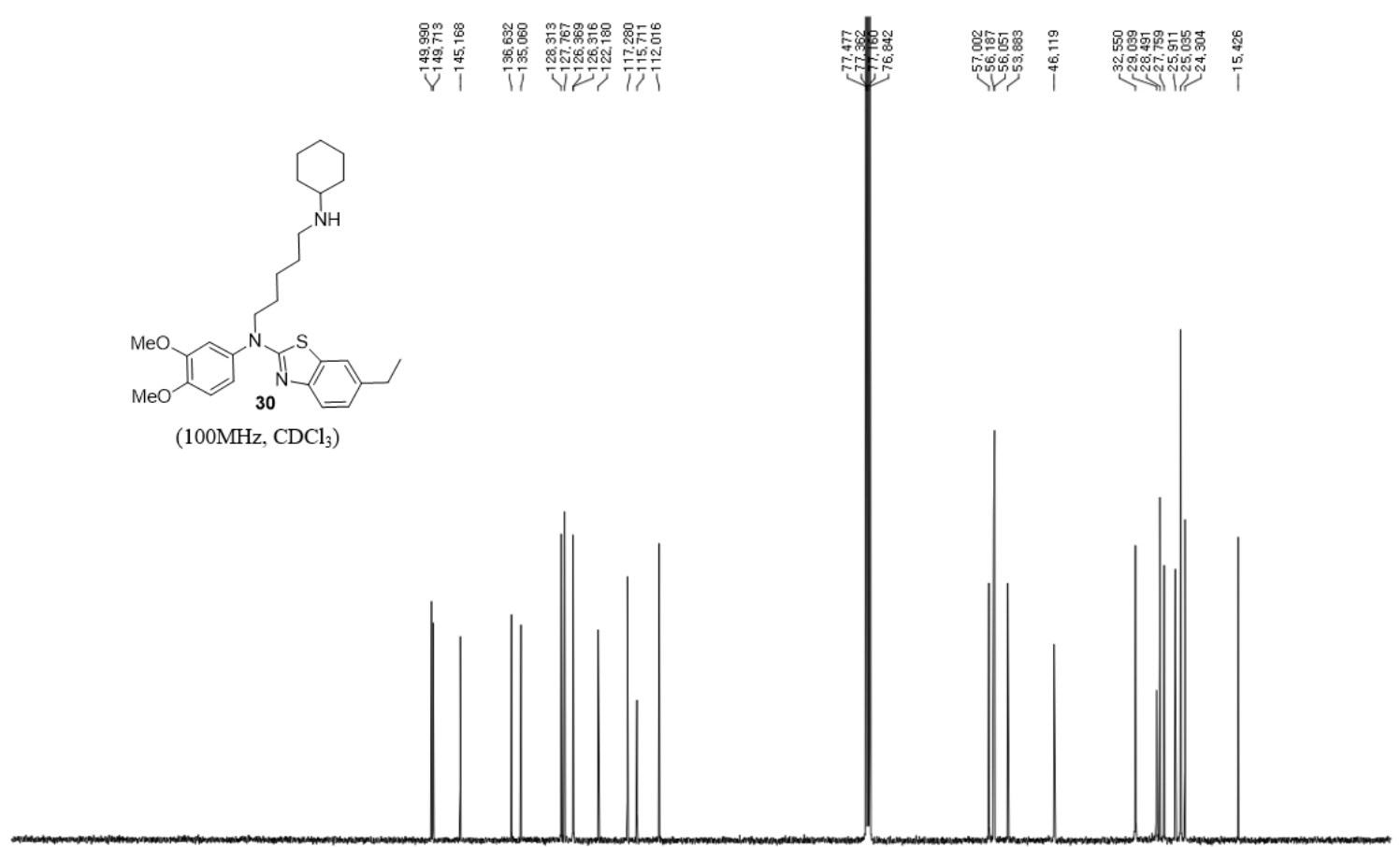

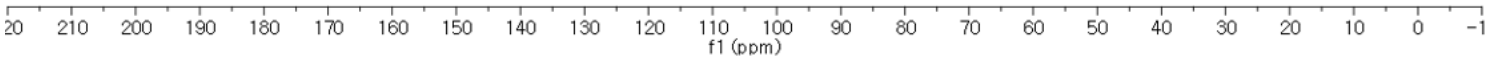

${ }^{13} \mathrm{C}$ NMR spectrum $(100 \mathrm{MHz})$ of $\mathbf{3 0}$ in $\mathrm{CDCl}_{3}$ 

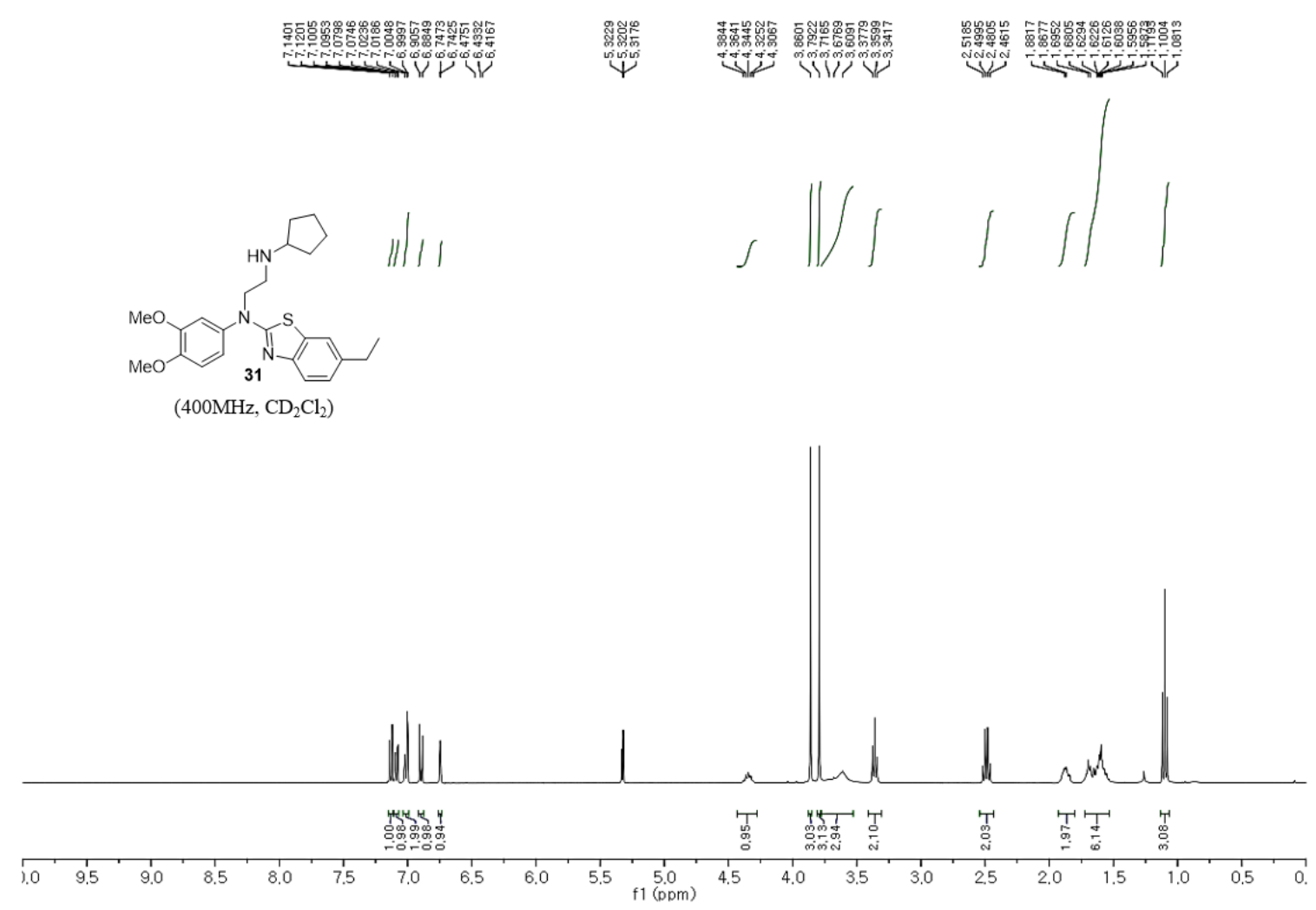

${ }^{1} \mathrm{H}$ NMR spectrum (400 MHz) of $\mathbf{3 1}$ in $\mathrm{CD}_{2} \mathrm{Cl}_{2}$

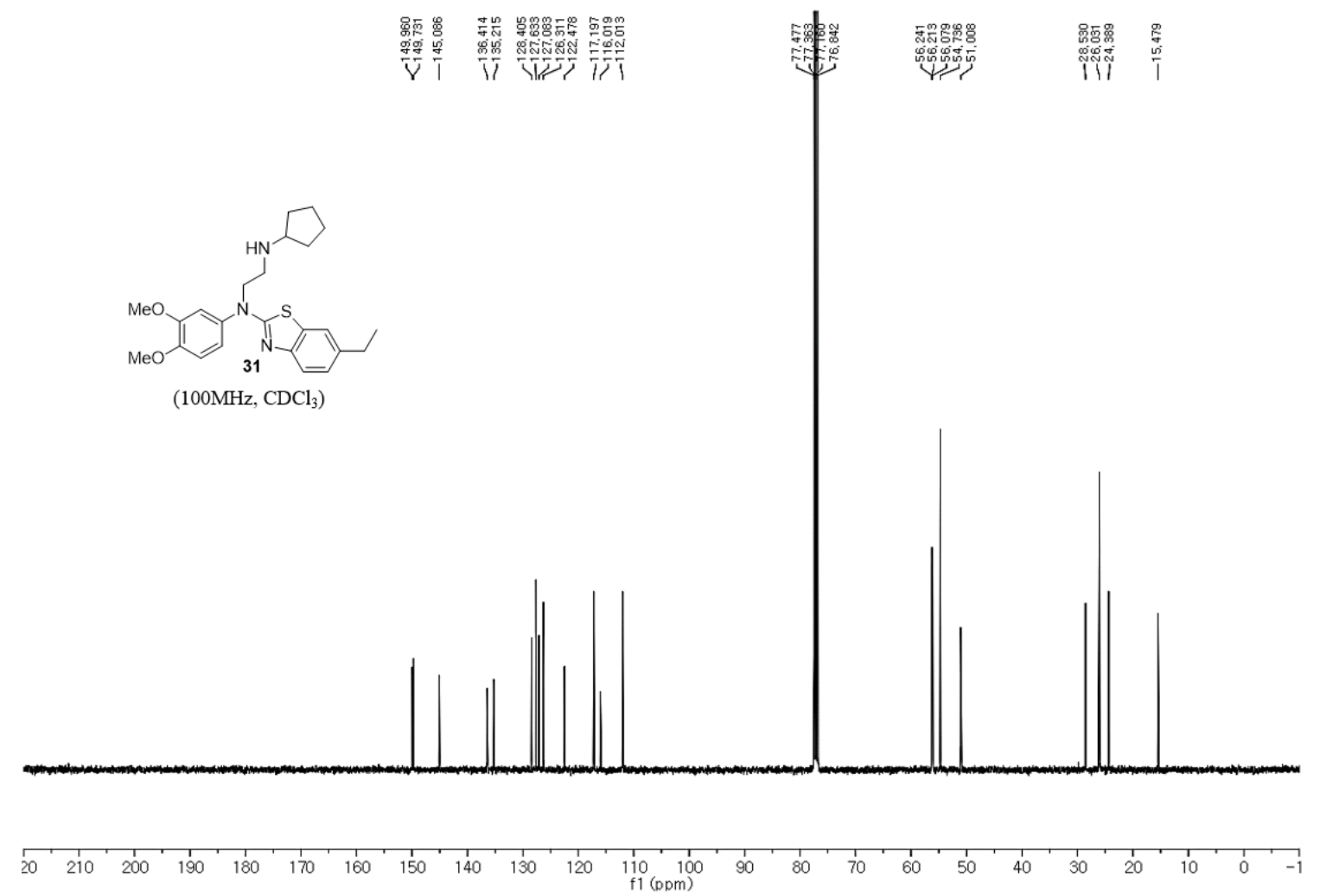

${ }^{13} \mathrm{C}$ NMR spectrum $(100 \mathrm{MHz})$ of $\mathbf{3 1}$ in $\mathrm{CDCl}_{3}$ 

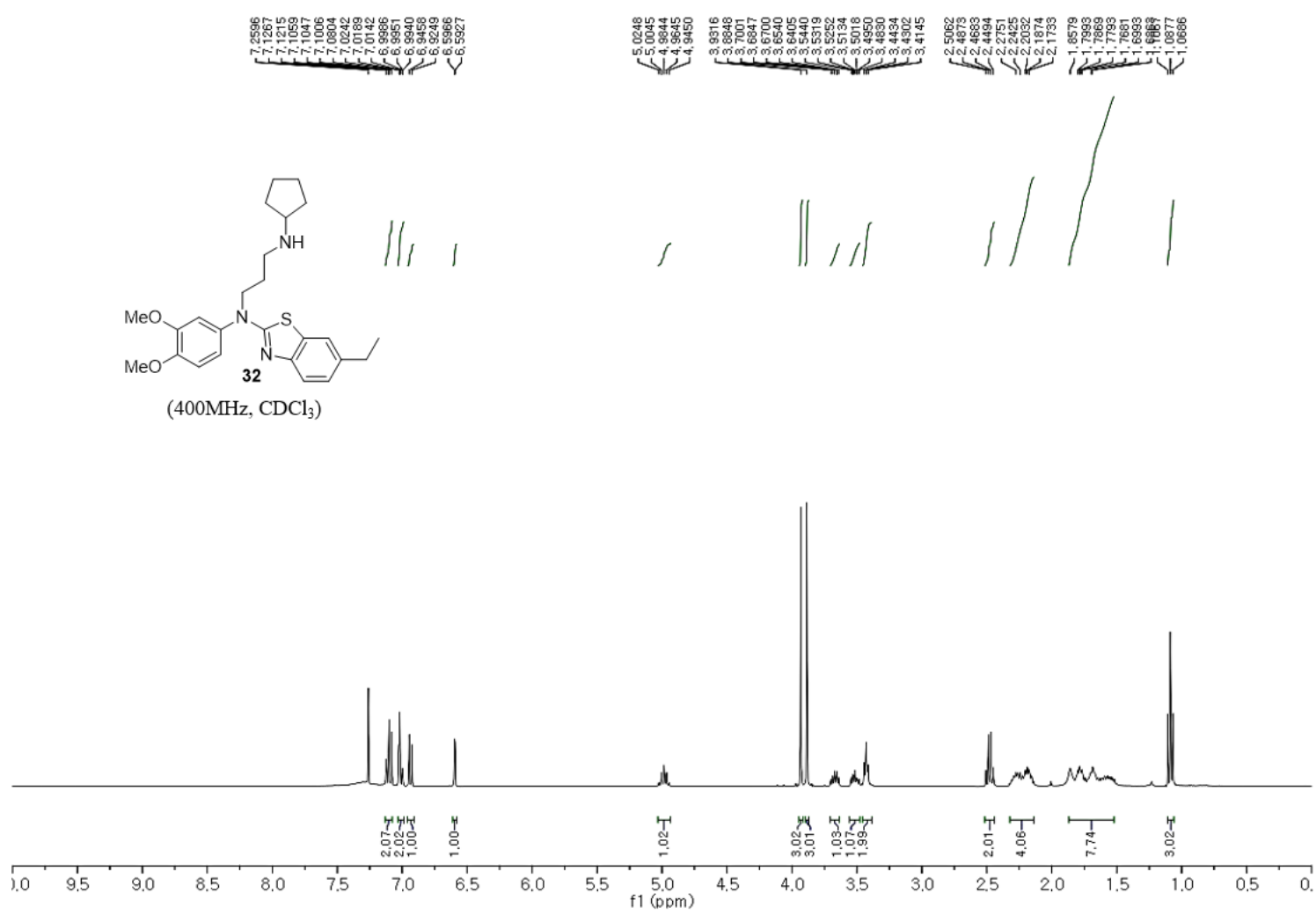

${ }^{1} \mathrm{H}$ NMR spectrum (400 MHz) of $\mathbf{3 2}$ in $\mathrm{CDCl}_{3}$

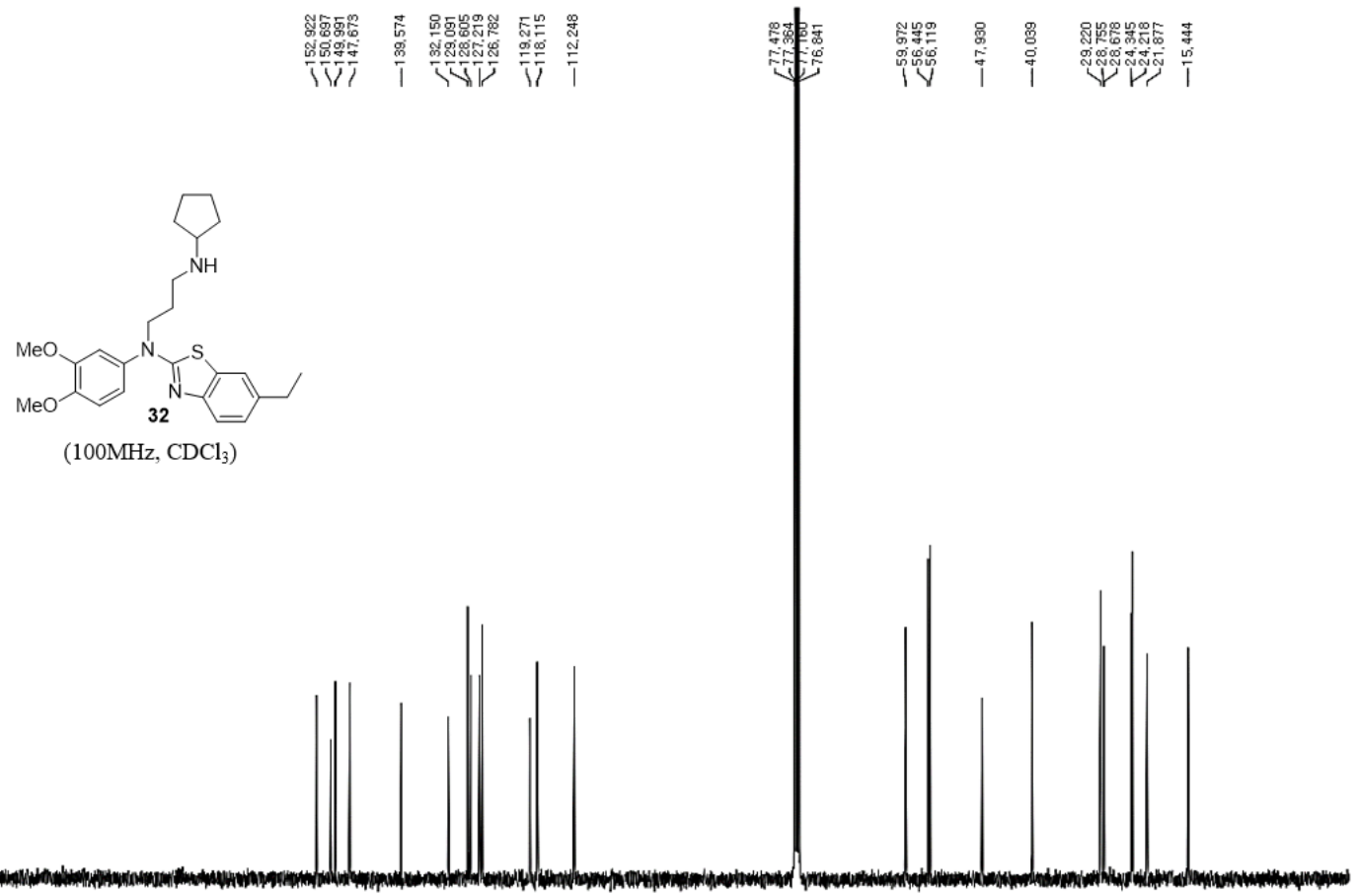

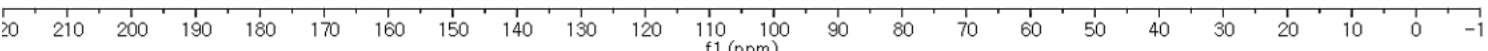

${ }^{13} \mathrm{C}$ NMR spectrum $(100 \mathrm{MHz})$ of $\mathbf{3 2}$ in $\mathrm{CDCl}_{3}$ 


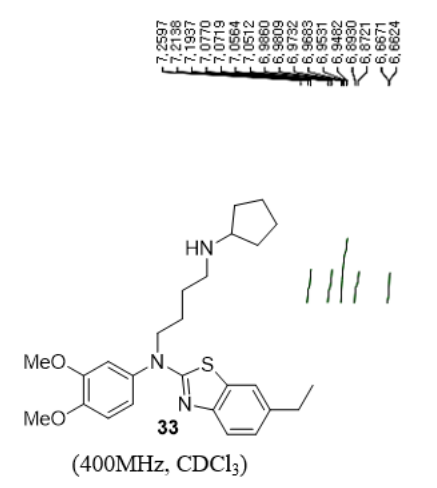

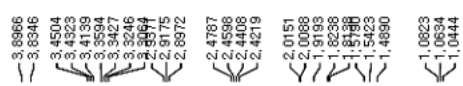

$\|\left.\operatorname{lil} 1\right|_{1} ^{1} 1$

$\left(400 \mathrm{MHz}, \mathrm{CDCl}_{3}\right)$

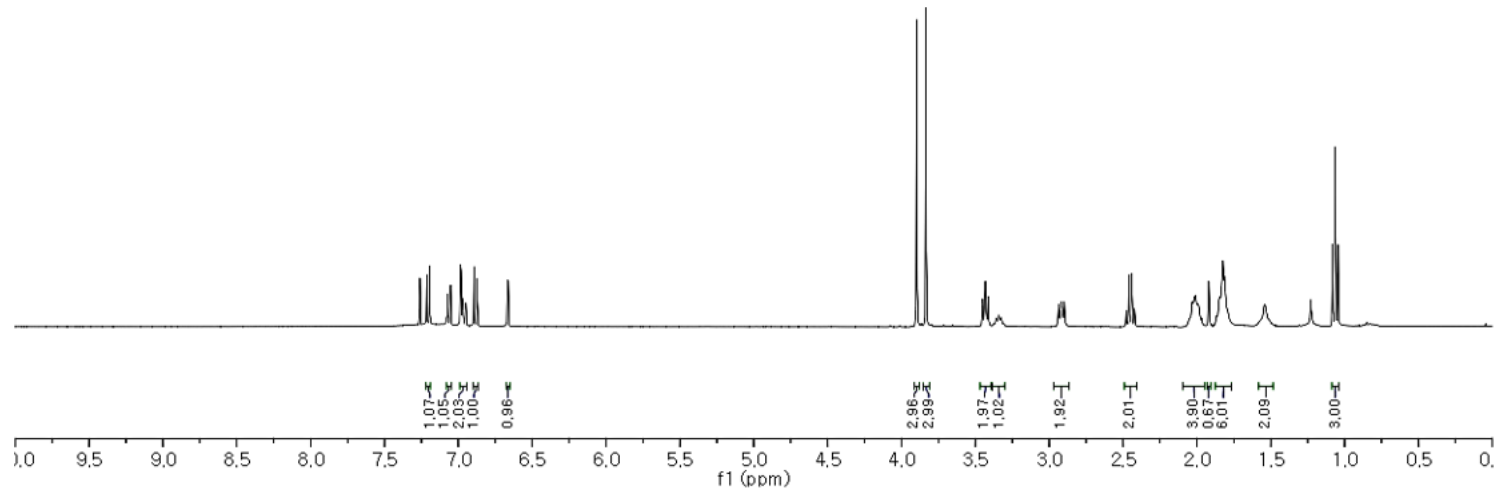

${ }^{1} \mathrm{H}$ NMR spectrum (400 MHz) of 33 in $\mathrm{CDCl}_{3}$

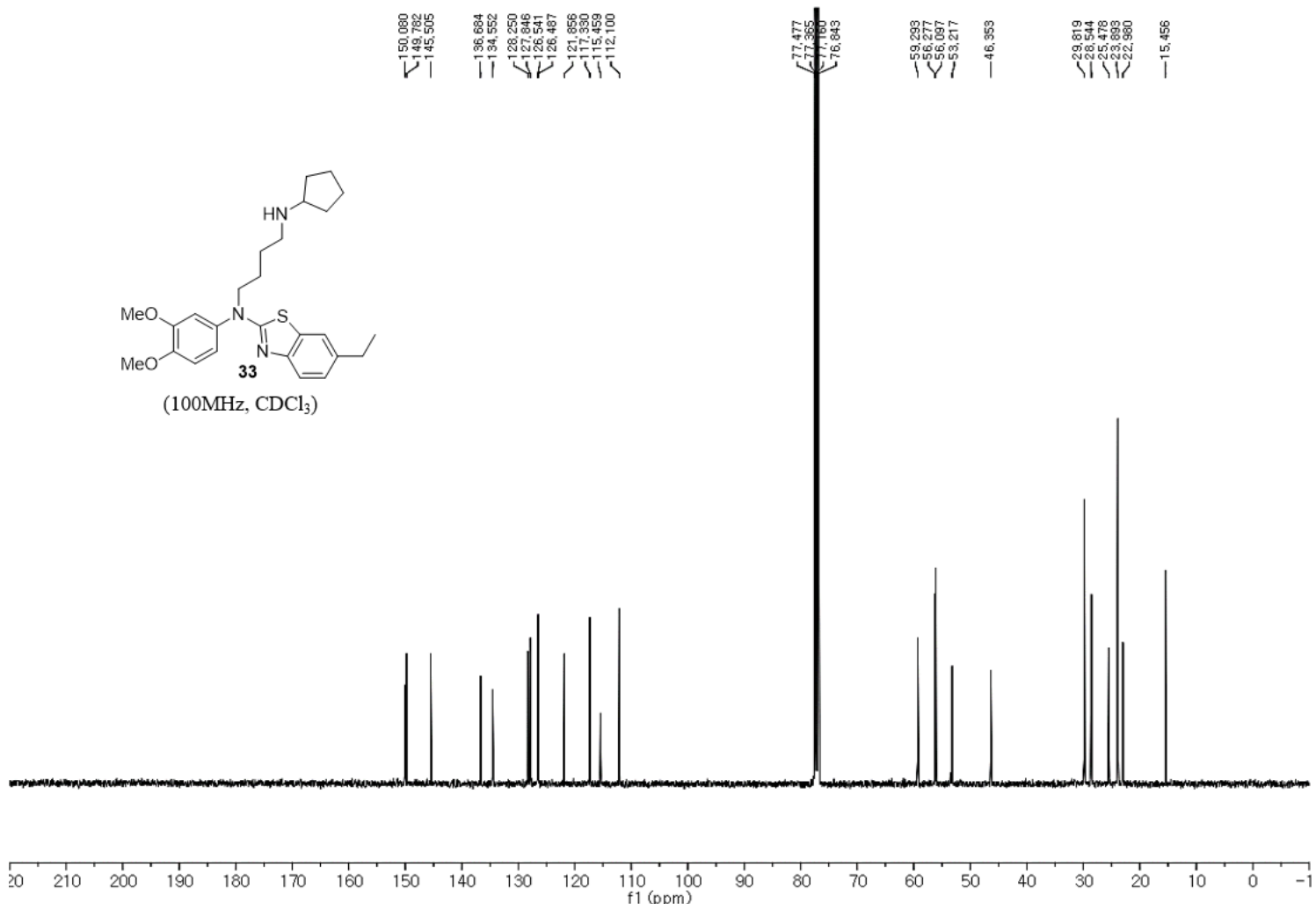

${ }^{13} \mathrm{C}$ NMR spectrum $(100 \mathrm{MHz})$ of $\mathbf{3 3}$ in $\mathrm{CDCl}_{3}$ 

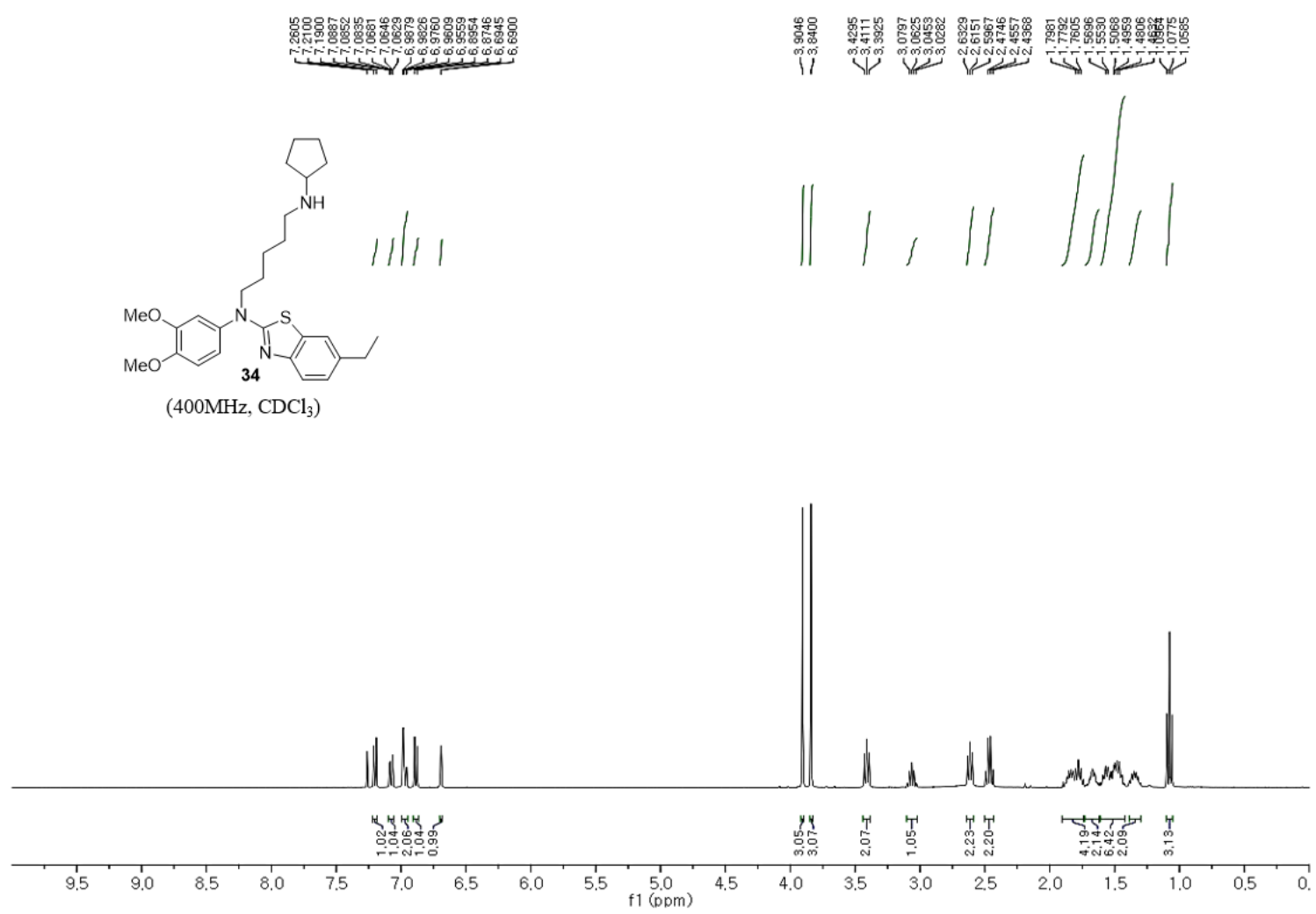

${ }^{1} \mathrm{H}$ NMR spectrum (400 MHz) of $\mathbf{3 4}$ in $\mathrm{CDCl}_{3}$

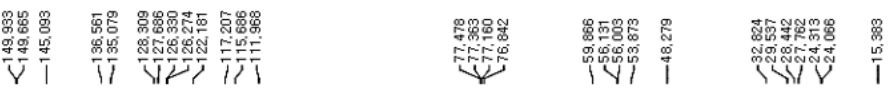
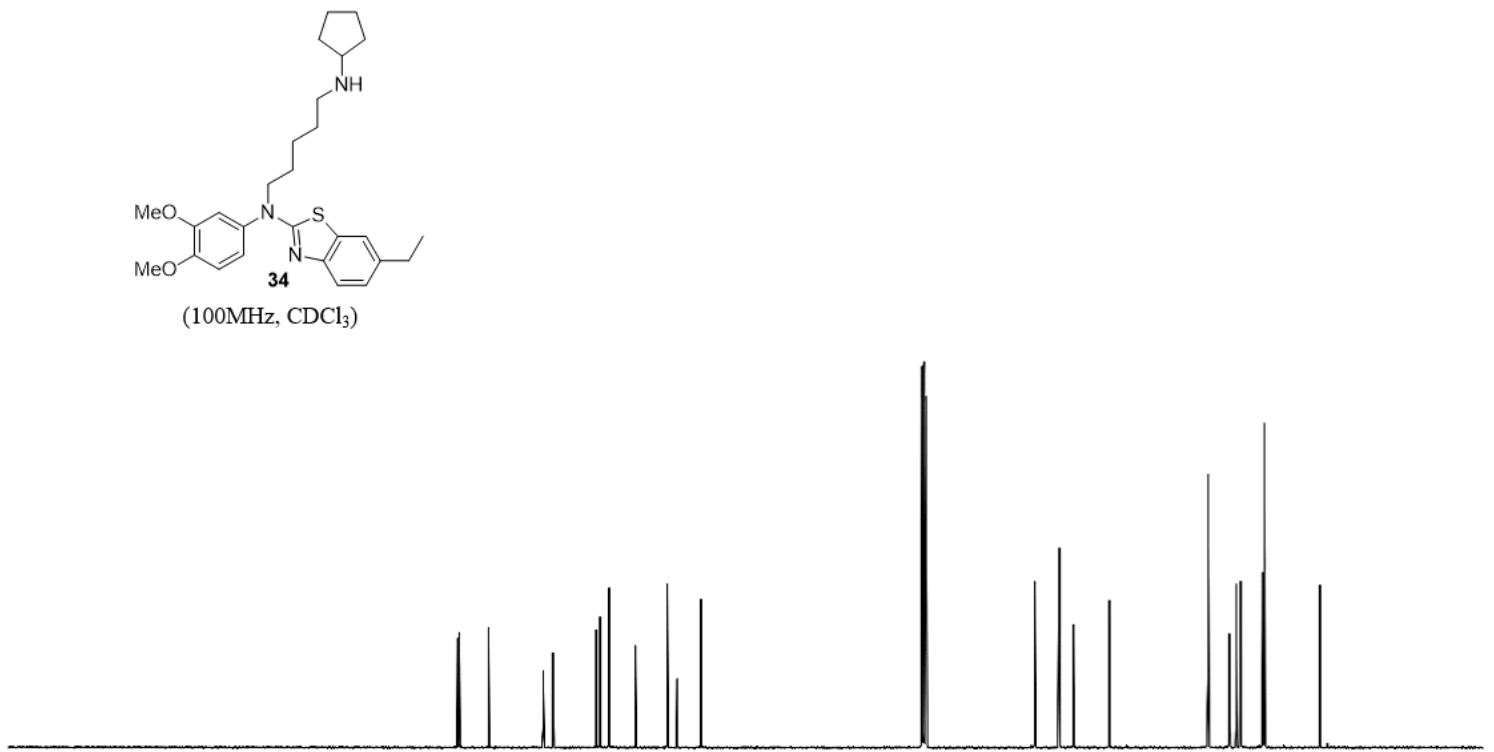

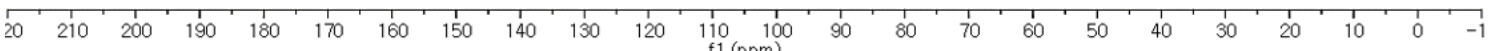

${ }^{13} \mathrm{C}$ NMR spectrum (100 MHz) of $\mathbf{3 4}$ in $\mathrm{CDCl}_{3}$ 

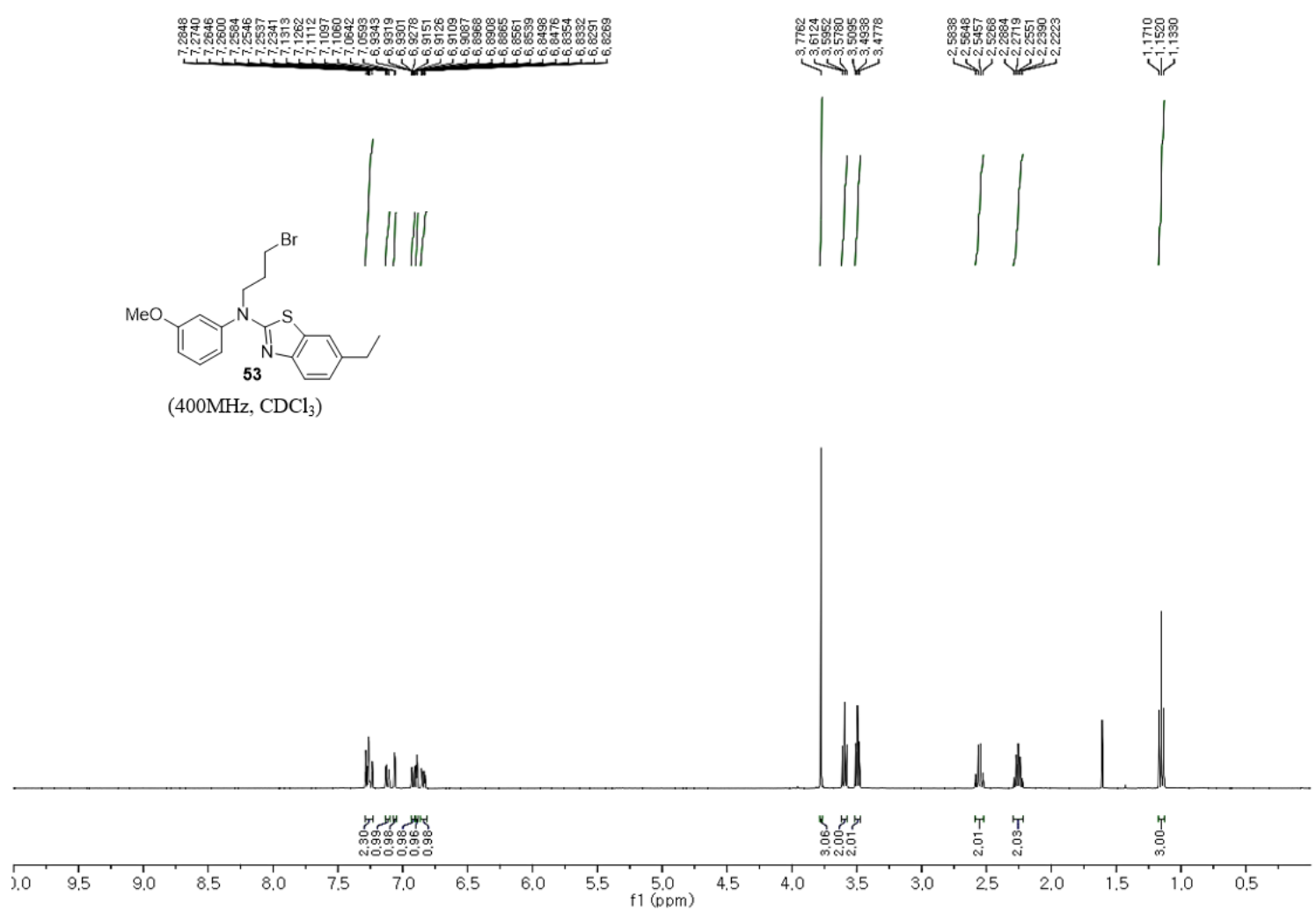

${ }^{1} \mathrm{H}$ NMR spectrum (400 MHz) of $\mathbf{5 3}$ in $\mathrm{CDCl}_{3}$

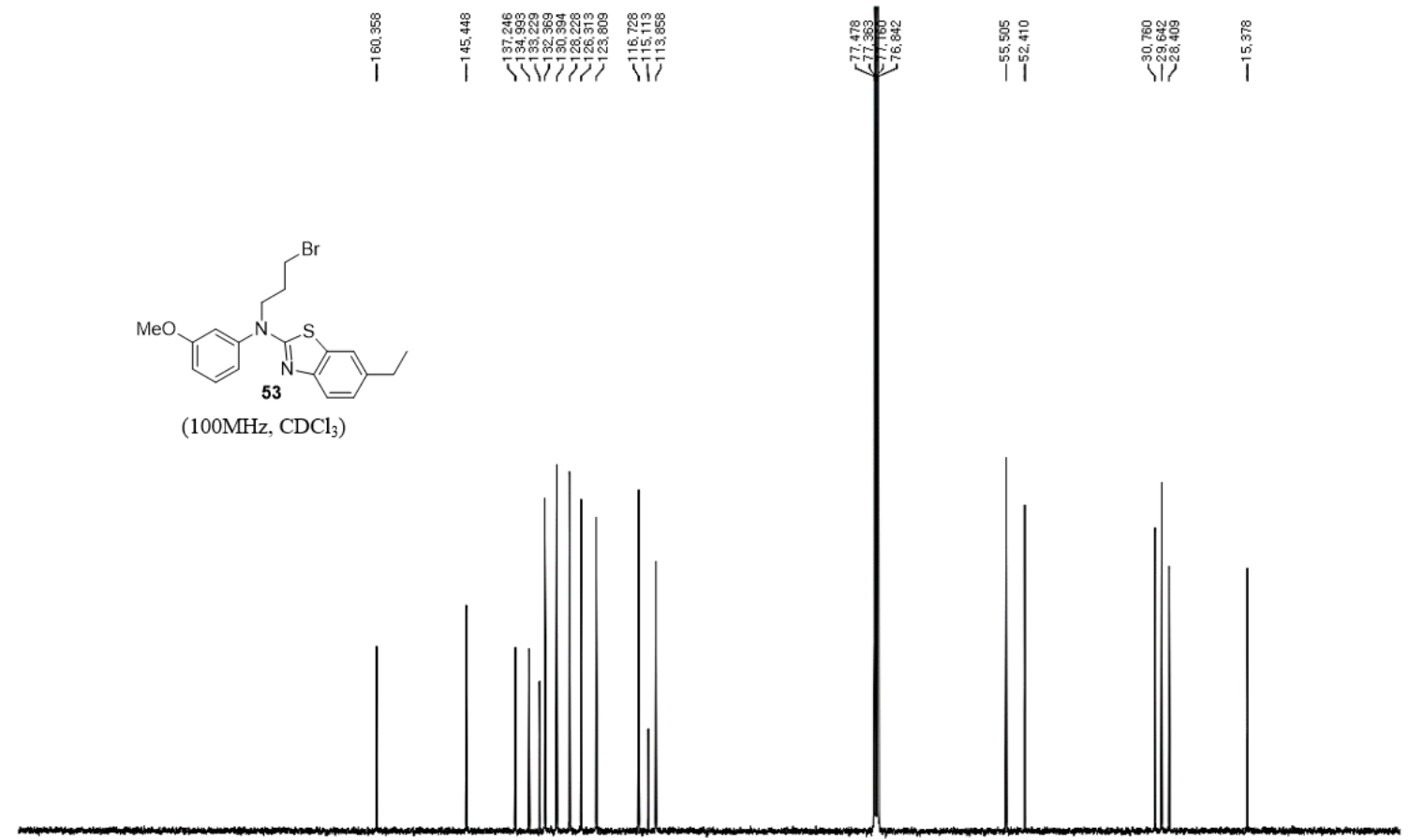

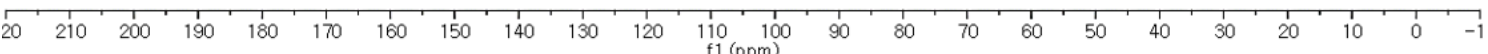

${ }^{13} \mathrm{C}$ NMR spectrum $(100 \mathrm{MHz})$ of $\mathbf{5 3}$ in $\mathrm{CDCl}_{3}$ 


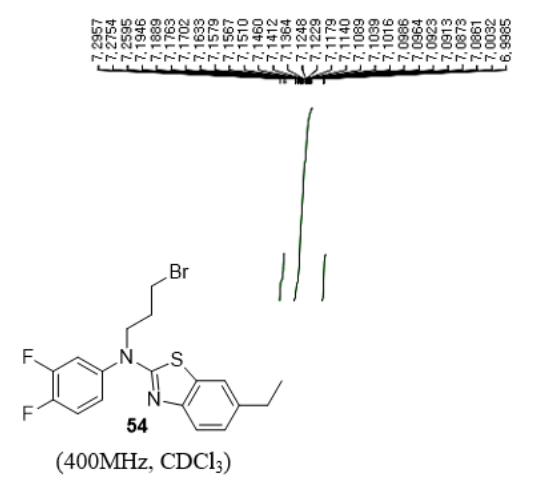

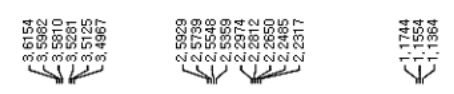
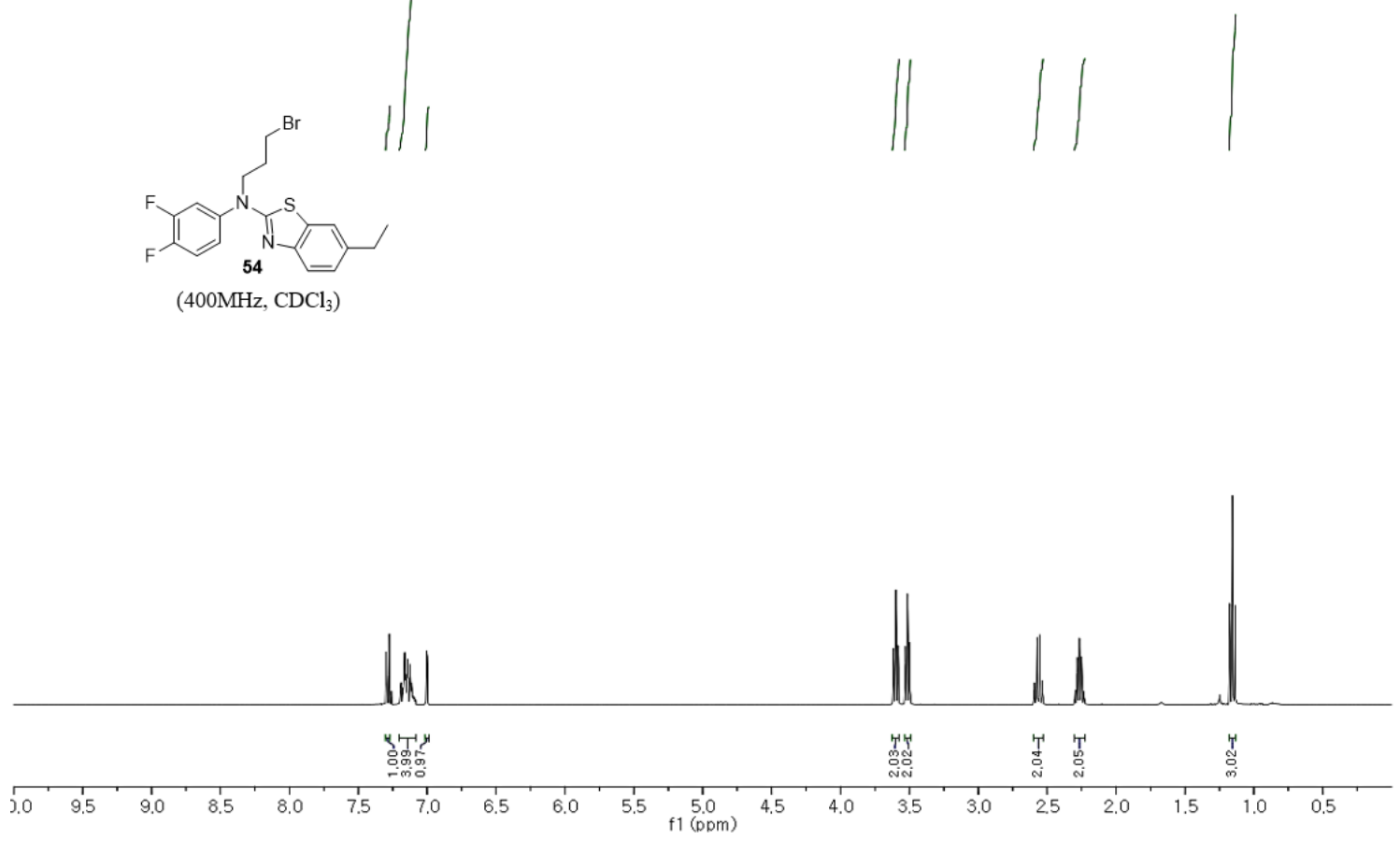

${ }^{1} \mathrm{H}$ NMR spectrum (400 MHz) of $\mathbf{5 4}$ in $\mathrm{CDCl}_{3}$

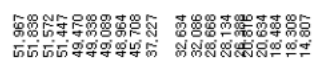

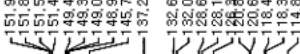

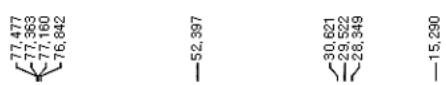
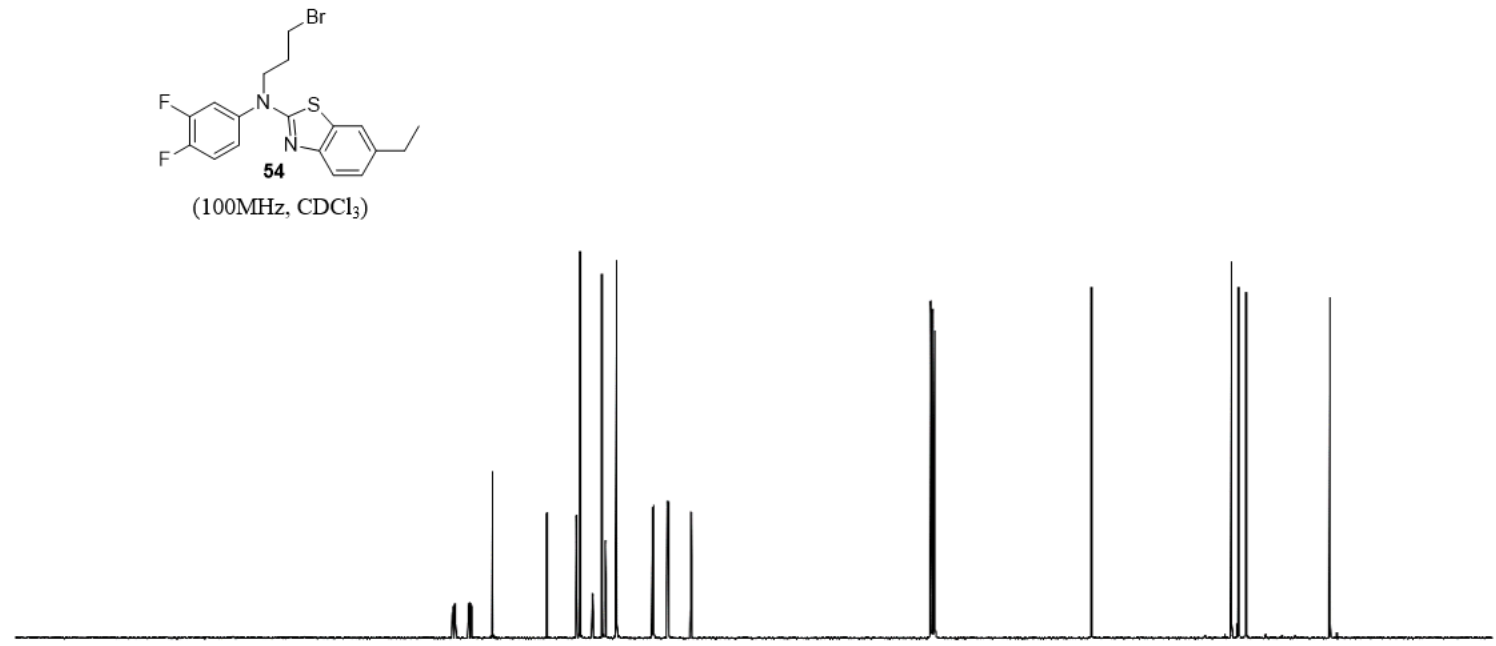

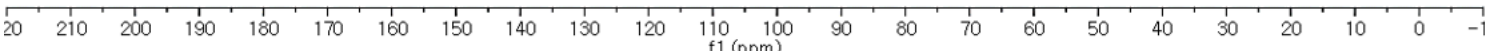

${ }^{13} \mathrm{C}$ NMR spectrum $(100 \mathrm{MHz})$ of 54 in $\mathrm{CDCl}_{3}$ 

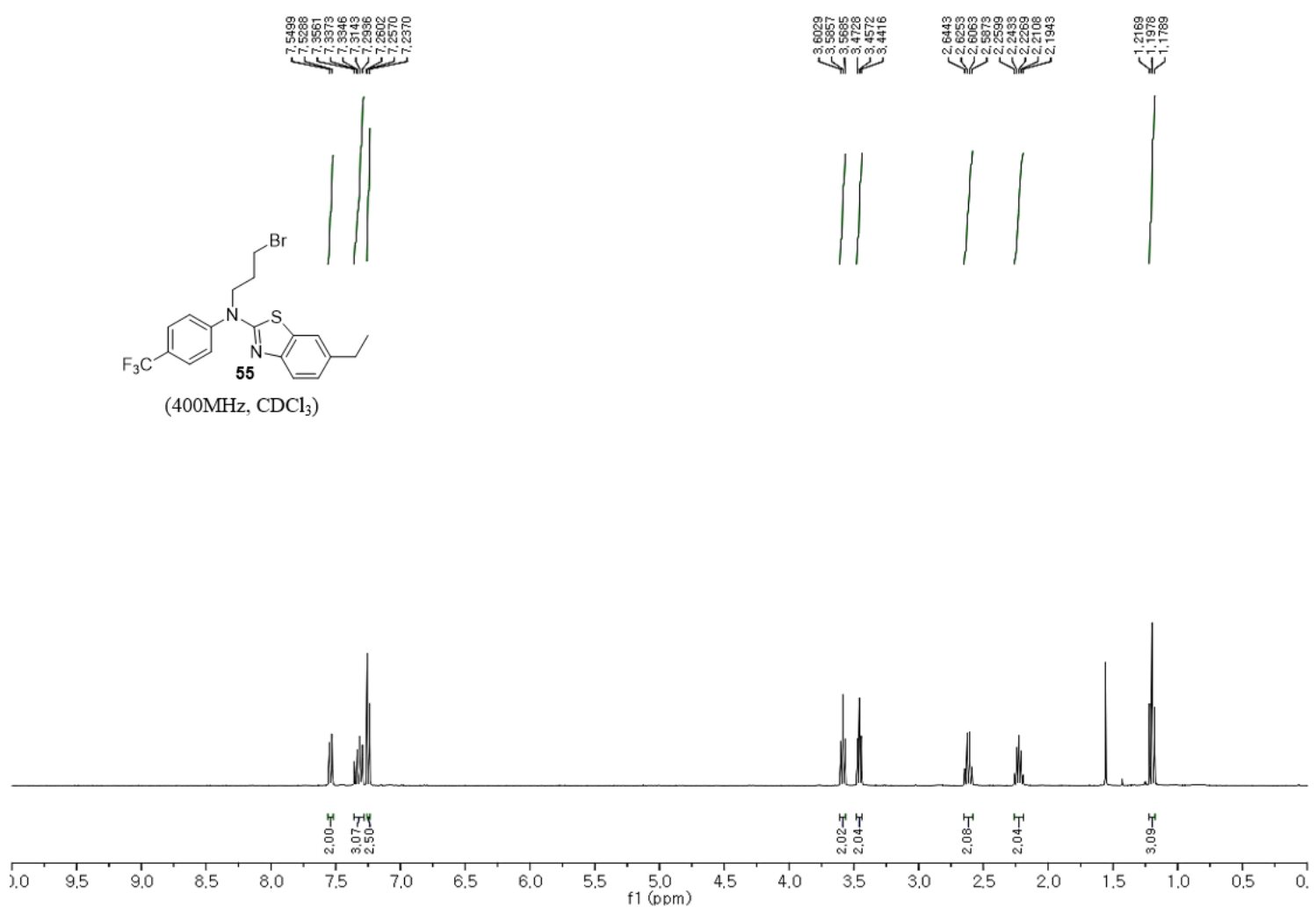

${ }^{1} \mathrm{H}$ NMR spectrum (400 MHz) of $\mathbf{5 5}$ in $\mathrm{CDCl}_{3}$
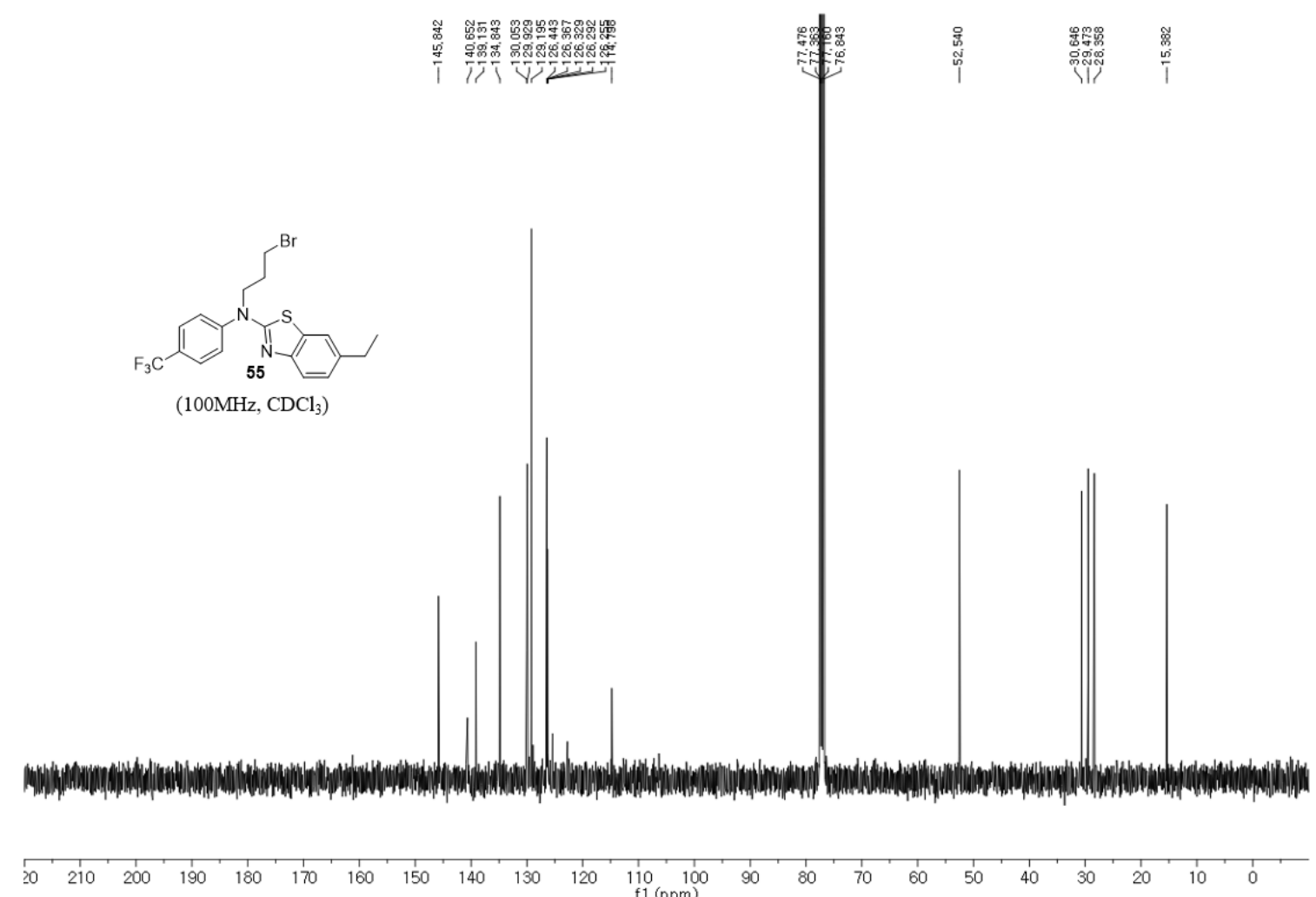

${ }^{13} \mathrm{C}$ NMR spectrum $(100 \mathrm{MHz})$ of $\mathbf{5 5}$ in $\mathrm{CDCl}_{3}$ 


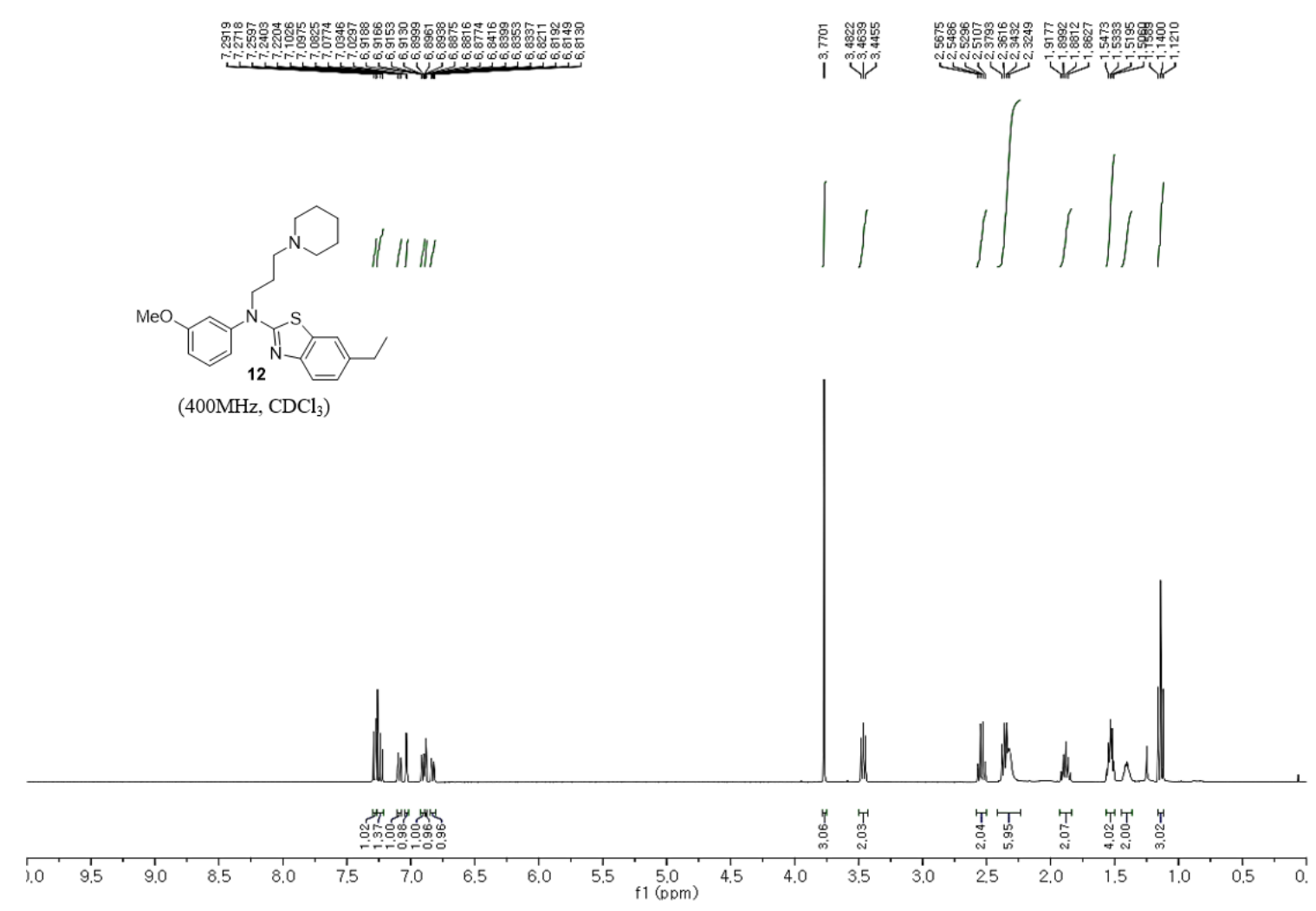

${ }^{1} \mathrm{H}$ NMR spectrum (400 MHz) of $\mathbf{1 2}$ in $\mathrm{CDCl}_{3}$

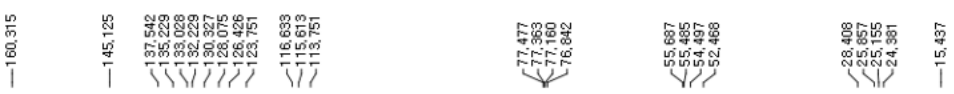

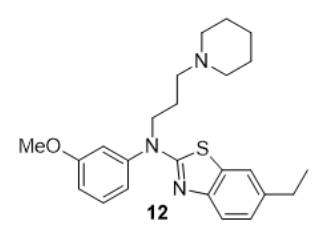

$\left(100 \mathrm{MHz}, \mathrm{CDCl}_{3}\right)$

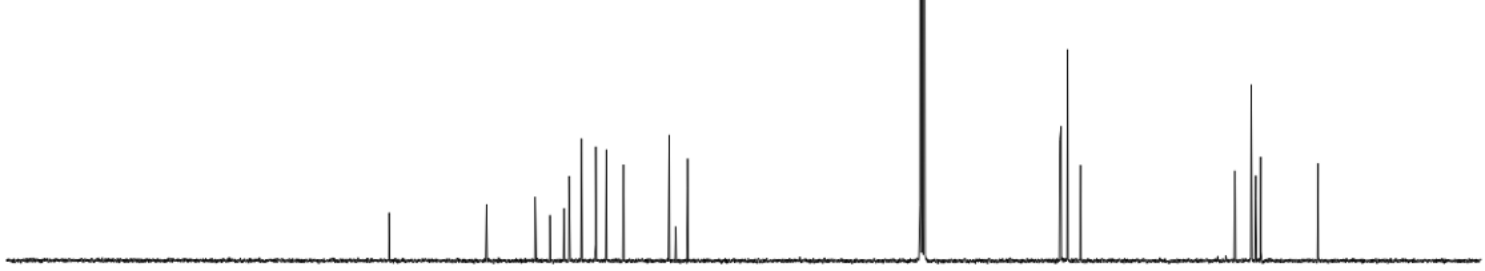

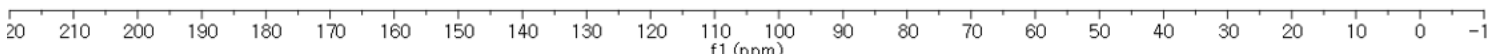

${ }^{13} \mathrm{C}$ NMR spectrum $(100 \mathrm{MHz})$ of $\mathbf{1 2}$ in $\mathrm{CDCl}_{3}$ 

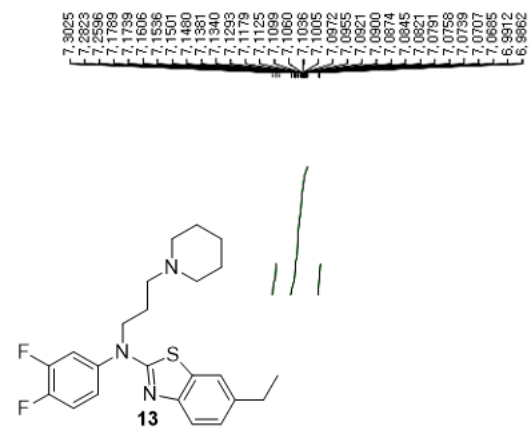

$\left(400 \mathrm{MHz}, \mathrm{CDCl}_{3}\right)$

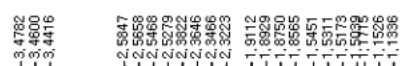

L"

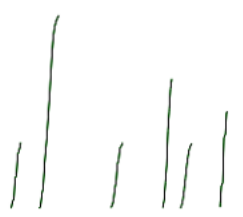

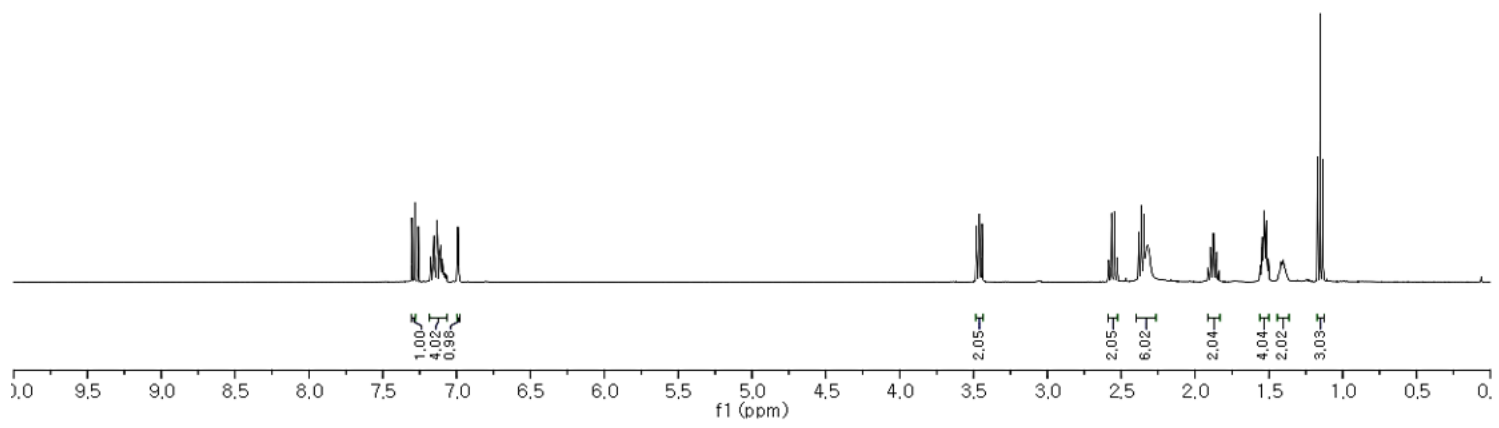

${ }^{1} \mathrm{H}$ NMR spectrum (400 MHz) of $\mathbf{1 3}$ in $\mathrm{CDCl}_{3}$

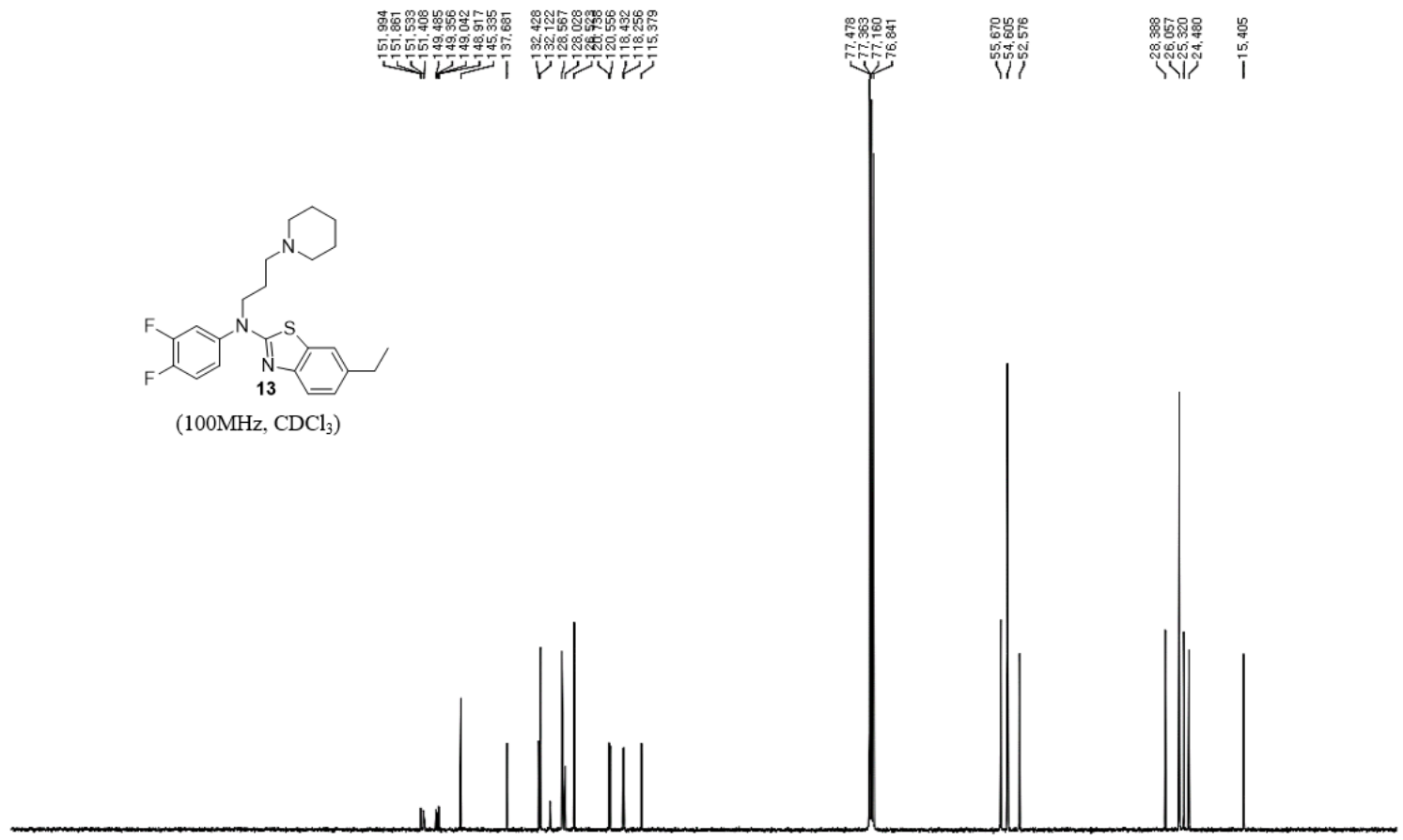

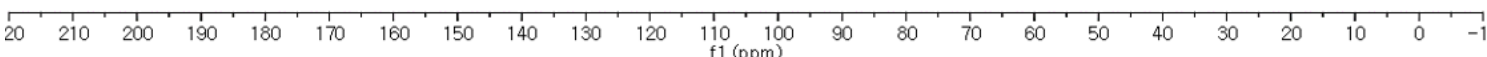

${ }^{13} \mathrm{C}$ NMR spectrum $(100 \mathrm{MHz})$ of $\mathbf{1 3}$ in $\mathrm{CDCl}_{3}$ 

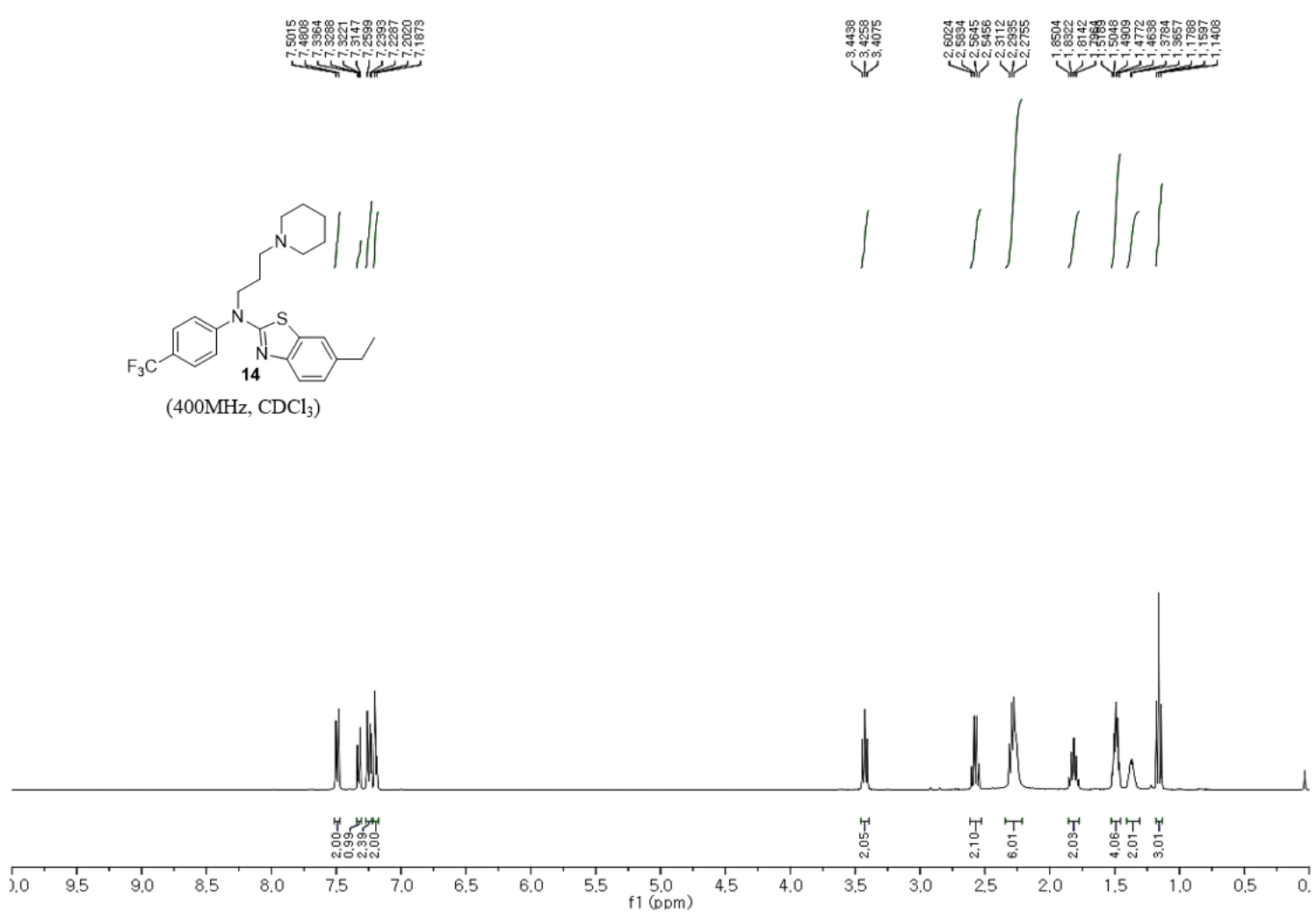

${ }^{1} \mathrm{H}$ NMR spectrum (400 MHz) of $\mathbf{1 4}$ in $\mathrm{CDCl}_{3}$

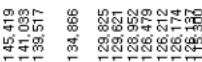

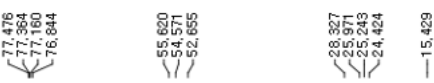

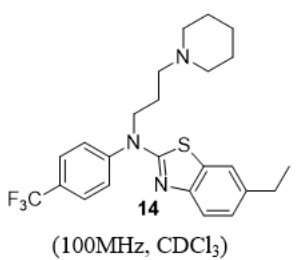

$\left(100 \mathrm{MHz}, \mathrm{CDCl}_{3}\right)$

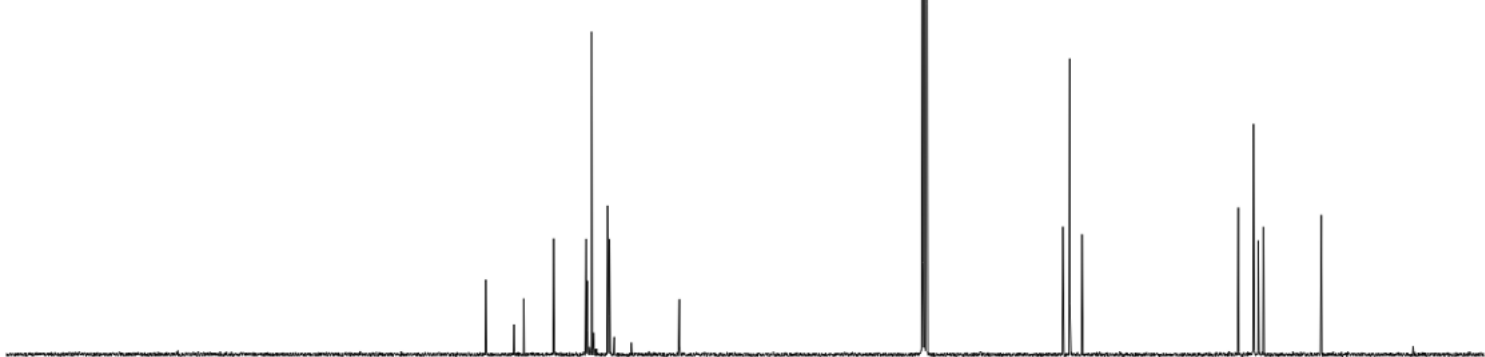

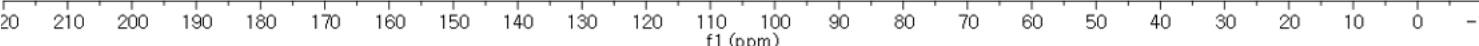

${ }^{13} \mathrm{C}$ NMR spectrum $(100 \mathrm{MHz})$ of 14 in $\mathrm{CDCl}_{3}$ 


\section{HRMS Analysis of Final Target Compounds}

$\mathrm{N}$-(3,4-Dimethoxyphenyl)-6-ethyl- $\mathrm{N}$-((1-(2-(piperidin-1-yl)ethyl)-1H-1,2,3-triazol-4yl)methyl)benzo[ $d]$ thiazol-2-amine (4)

HRMS (FAB): calcd. for $\mathrm{C}_{27} \mathrm{H}_{35} \mathrm{~N}_{6} \mathrm{O}_{2} \mathrm{~S}[\mathrm{M}+\mathrm{H}]^{+}$507.2542, found 507.2539.

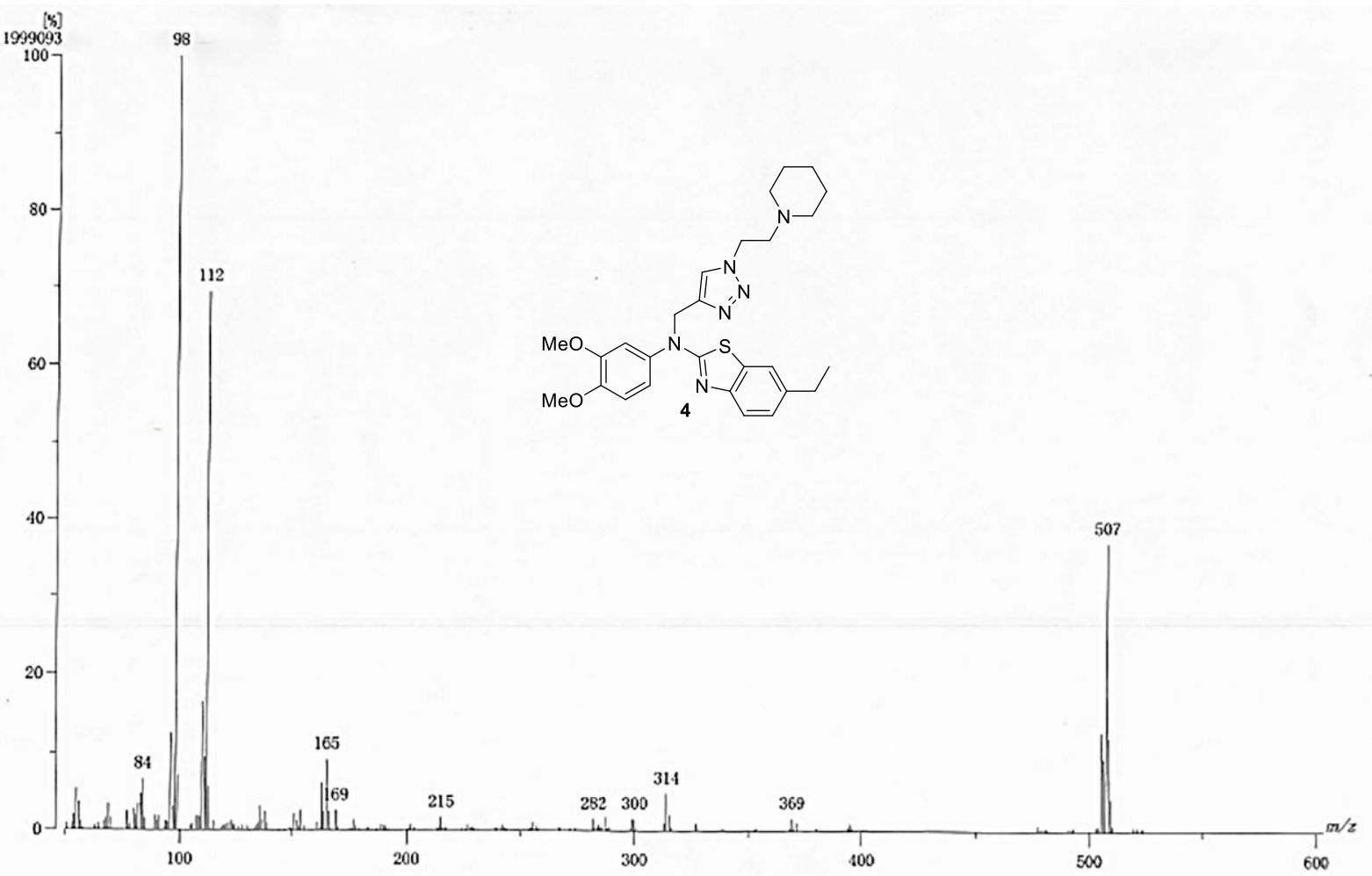

[ Theoretical Ion Distribution ]

Molecular Formula : C27 H35 N6 O2 S

$(\mathrm{m} / \mathrm{z} \quad 507.2542$, MW 507.6799 , U.S. 14.5)

Base Peak : 507.2542, Averaged MW : $507.6752(\mathrm{a}), \quad 507.6762(\mathrm{w})$

$\mathrm{m} / \mathrm{z} \quad$ INT.

$507.2542100 .0000 *$

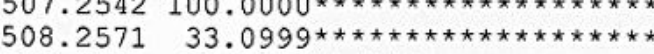

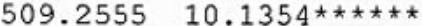

$510.25602 .1121 *$

$511.2571 \quad 0.3252$

$512.2584 \quad 0.0397$

$513.2600 \quad 0.0040$

$514.2617 \quad 0.0003$

\begin{tabular}{|c|c|c|c|c|c|c|c|c|c|}
\hline Observed $\mathrm{m} / \mathrm{z}$ & Int $\%$ & Err [ppm & / $\mathrm{mmu}]$ & U.S. & Com & os i & ior & & \\
\hline $1 \quad 507.2539$ & 100.00 & $+6.0 /$ & +3.1 & 18.5 & C 30 & H31 & N6 & 02 & \\
\hline (2) & & $+3.4 /$ & +1.7 & 18.0 & C32 & $\mathrm{H} 33$ & N3 & 03 & \\
\hline 3 & & $+0.7 /$ & +0.4 & 17.5 & C34 & H35 & 04 & & \\
\hline 4 & & $-8.6 /$ & $-4 \cdot 3$ & 18.5 & C 32 & H35 & N4 & S & \\
\hline 5 & & $-0.6 /$ & -0.3 & 14.5 & C2 7 & H35 & N 6 & 02 & S \\
\hline 6 & & $-3.3 /$ & -1.7 & 14.0 & C29 & $\mathrm{H} 37$ & N3 & 03 & S \\
\hline 7 & & -5.9 & -3.0 & 13. 5 & C 31 & $\mathrm{H} 39$ & 04 & S & \\
\hline 8 & & $+4.6 /$ & +2.4 & 10.0 & C 24 & H37 & N5 & 05 & $S$ \\
\hline
\end{tabular}


$N$-((1-(2-(Cyclopentylamino)ethyl)-1H-1,2,3-triazol-4-yl)methyl)- $N$-(3,4-dimethoxyphenyl)-6ethylbenzo[d]thiazol-2-amine (5)

HRMS (FAB): calcd. for $\mathrm{C}_{27} \mathrm{H}_{35} \mathrm{~N}_{6} \mathrm{O}_{2} \mathrm{~S}[\mathrm{M}+\mathrm{H}]^{+}$507.2542, found 507.2552.

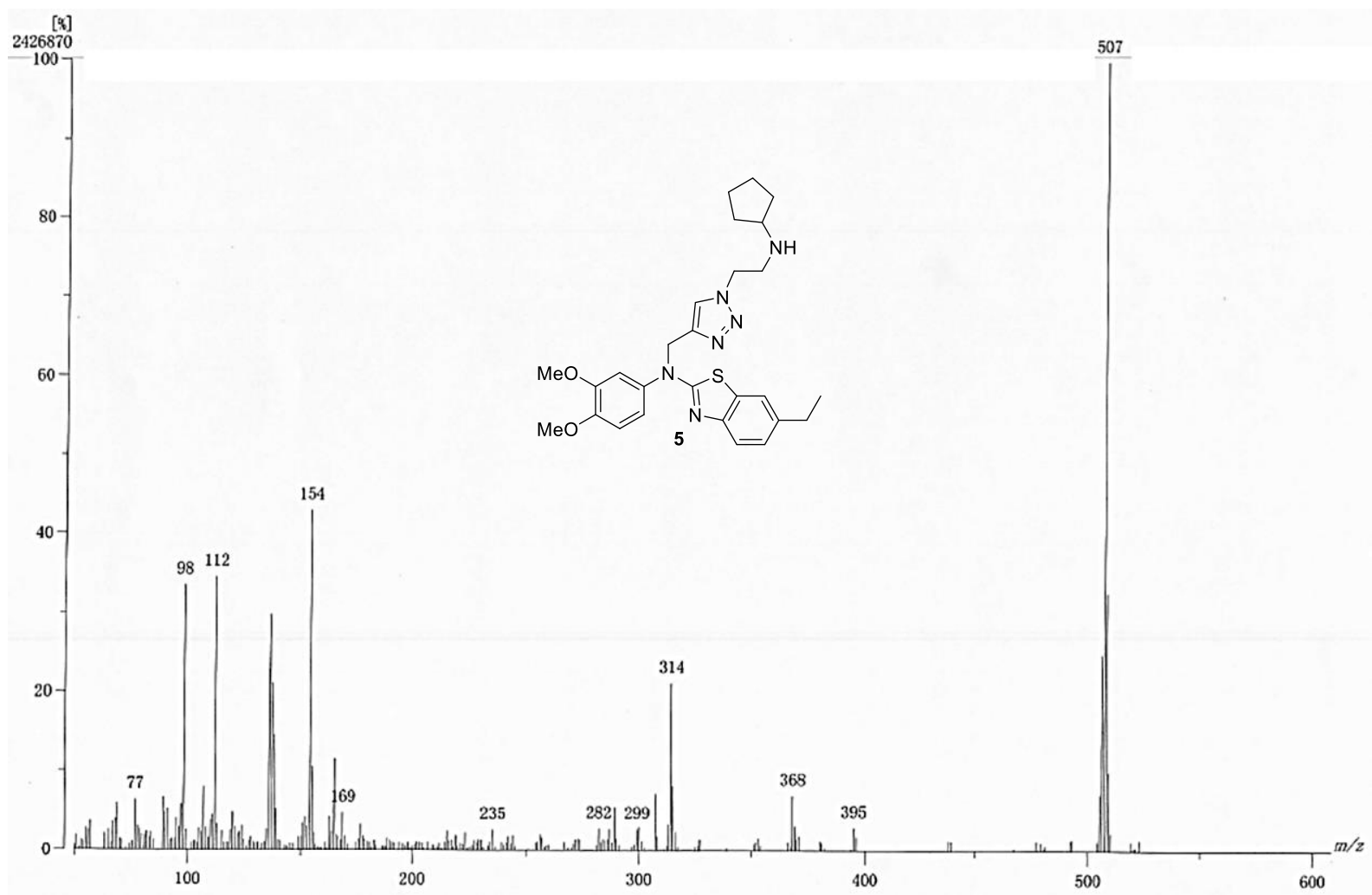

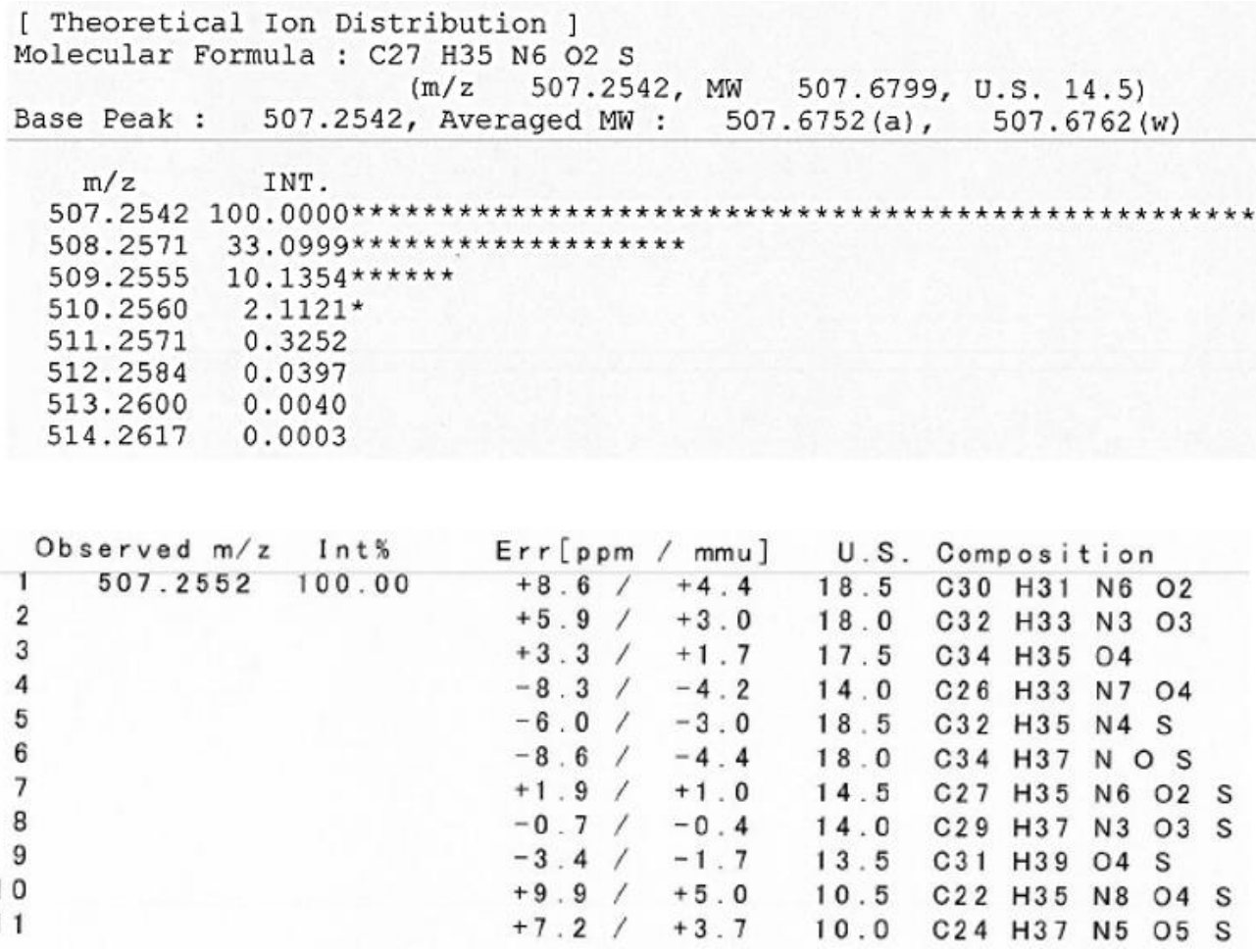


$N$-(3,4-Dimethoxyphenyl)-6-ethyl- $N$-((1-(2-(propylamino)ethyl)-1H-1,2,3-triazol-4yl)methyl)benzo[ $d]$ thiazol-2-amine (6)

HRMS (ESI): calcd. for $\mathrm{C}_{25} \mathrm{H}_{33} \mathrm{~N}_{6} \mathrm{O}_{2} \mathrm{~S}[\mathrm{M}+\mathrm{H}]^{+}$481.2380, found 481.2387 .

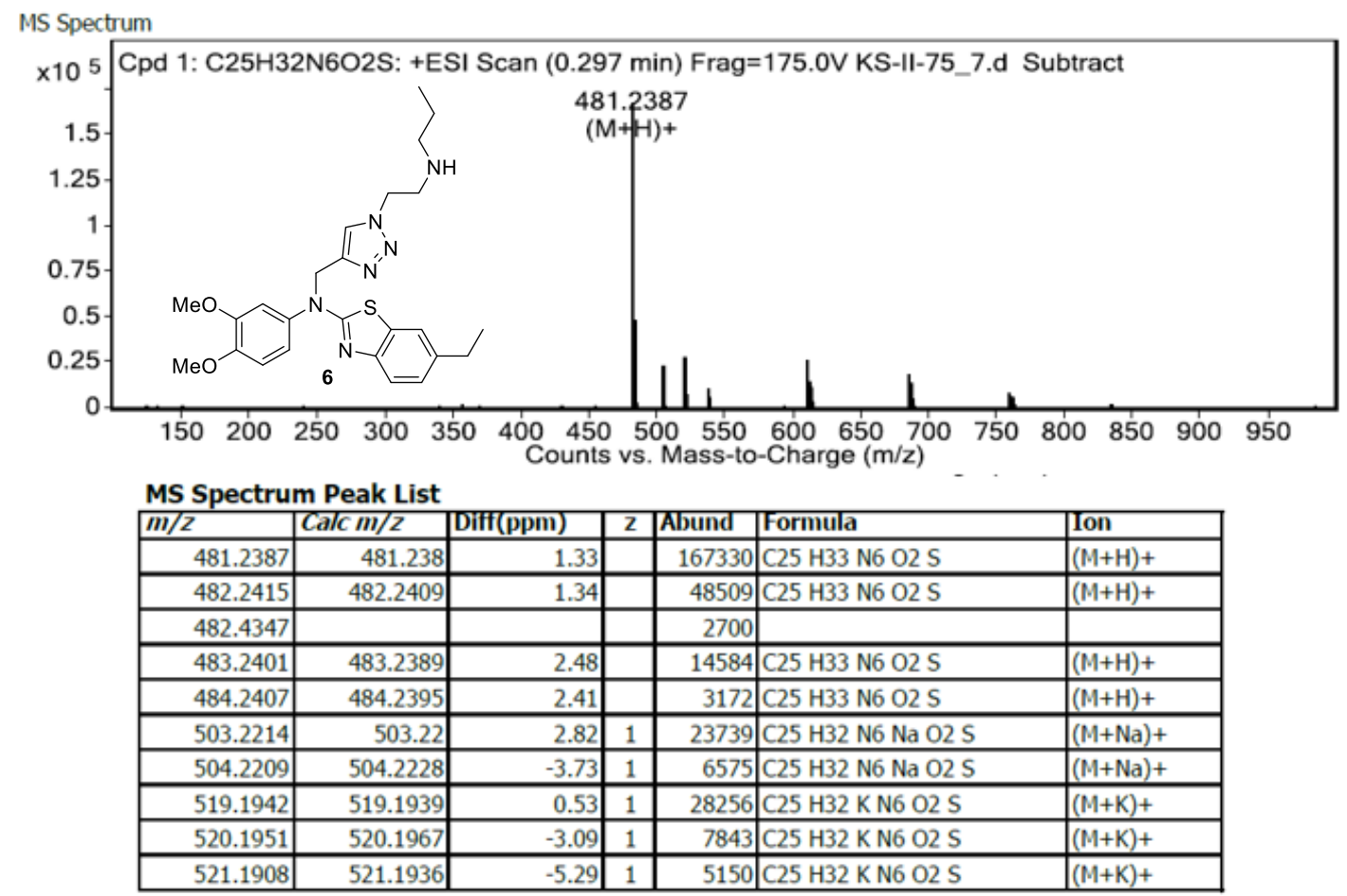


$N$-(3,4-Dimethoxyphenyl)-6-ethyl- $N$-((1-(2-(pyrrolidin-1-yl)ethyl)-1H-1,2,3-triazol-4yl)methyl)benzo[d]thiazol-2-amine (7)

HRMS (FAB): calcd. for $\mathrm{C}_{26} \mathrm{H}_{33} \mathrm{~N}_{6} \mathrm{O}_{2} \mathrm{~S}[\mathrm{M}+\mathrm{H}]^{+}$493.2386, found 493.2382.

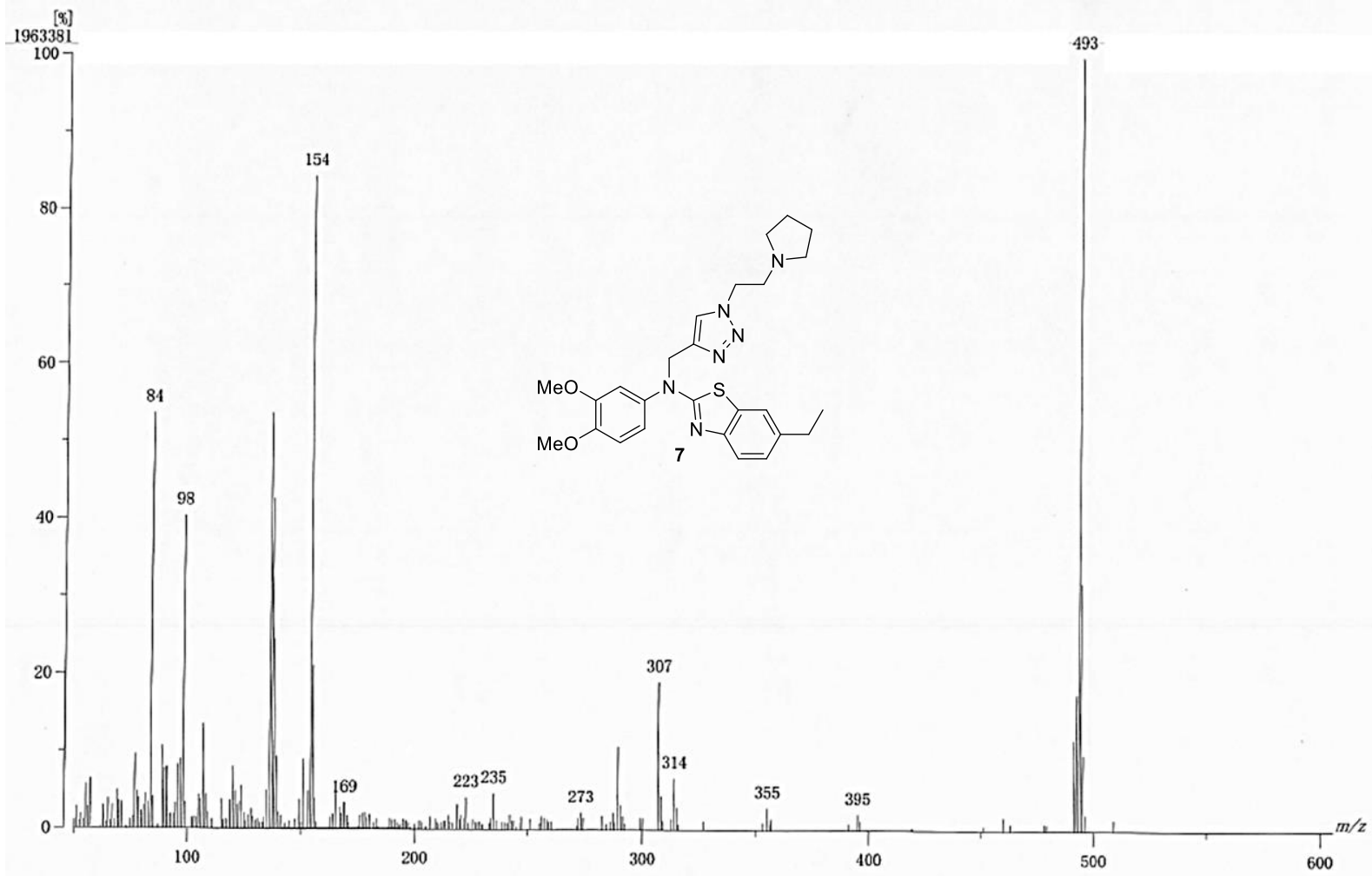

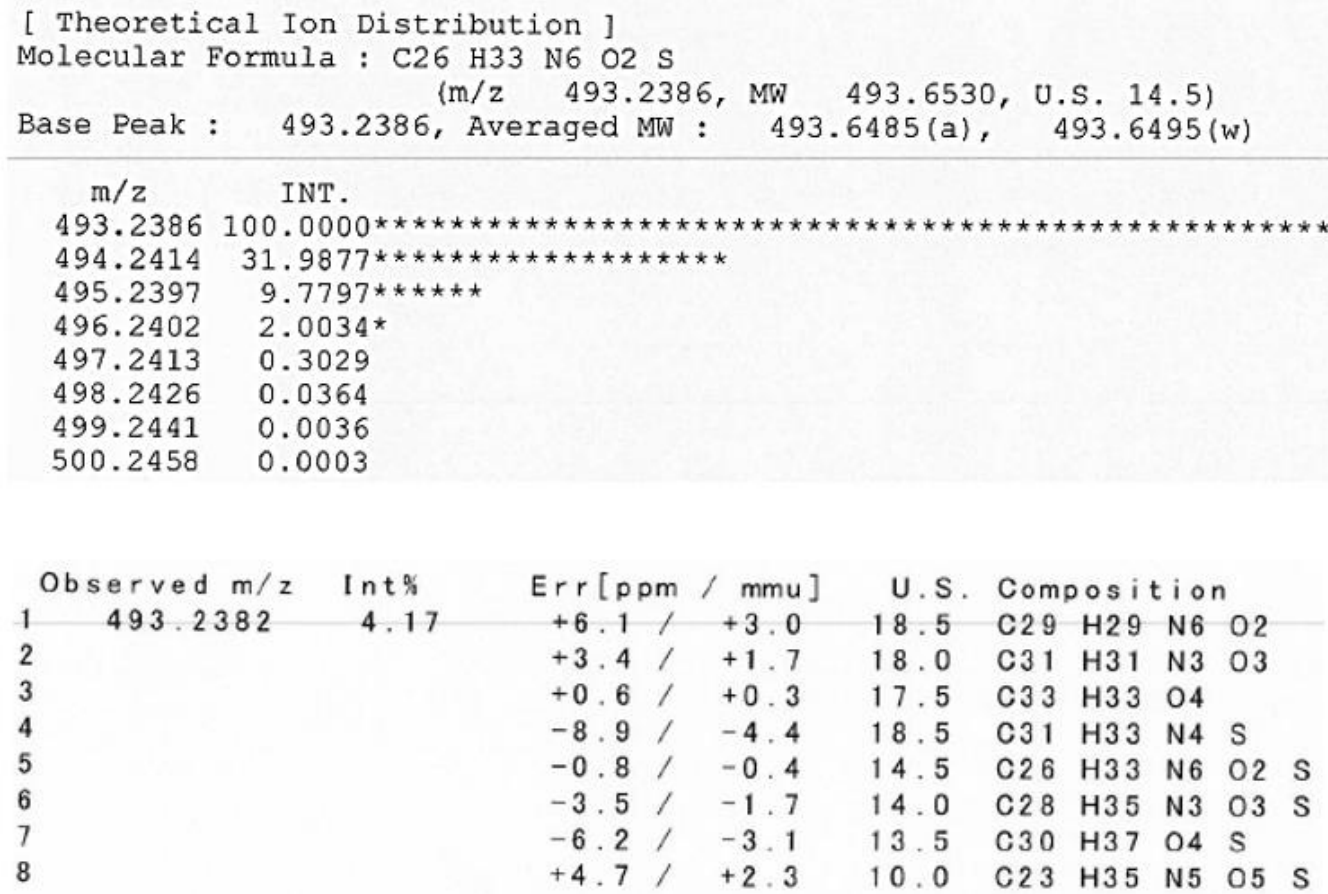


$N$-(3,4-Dimethoxyphenyl)-6-ethyl- $N$-(2-(piperidin-1-yl)ethyl)benzo[ $d]$ thiazol-2-amine (8)

HRMS (ESI): calcd. for $\mathrm{C}_{24} \mathrm{H}_{32} \mathrm{~N}_{3} \mathrm{O}_{2} \mathrm{~S}[\mathrm{M}+\mathrm{H}]^{+} 426.2210$, found 426.2204 .

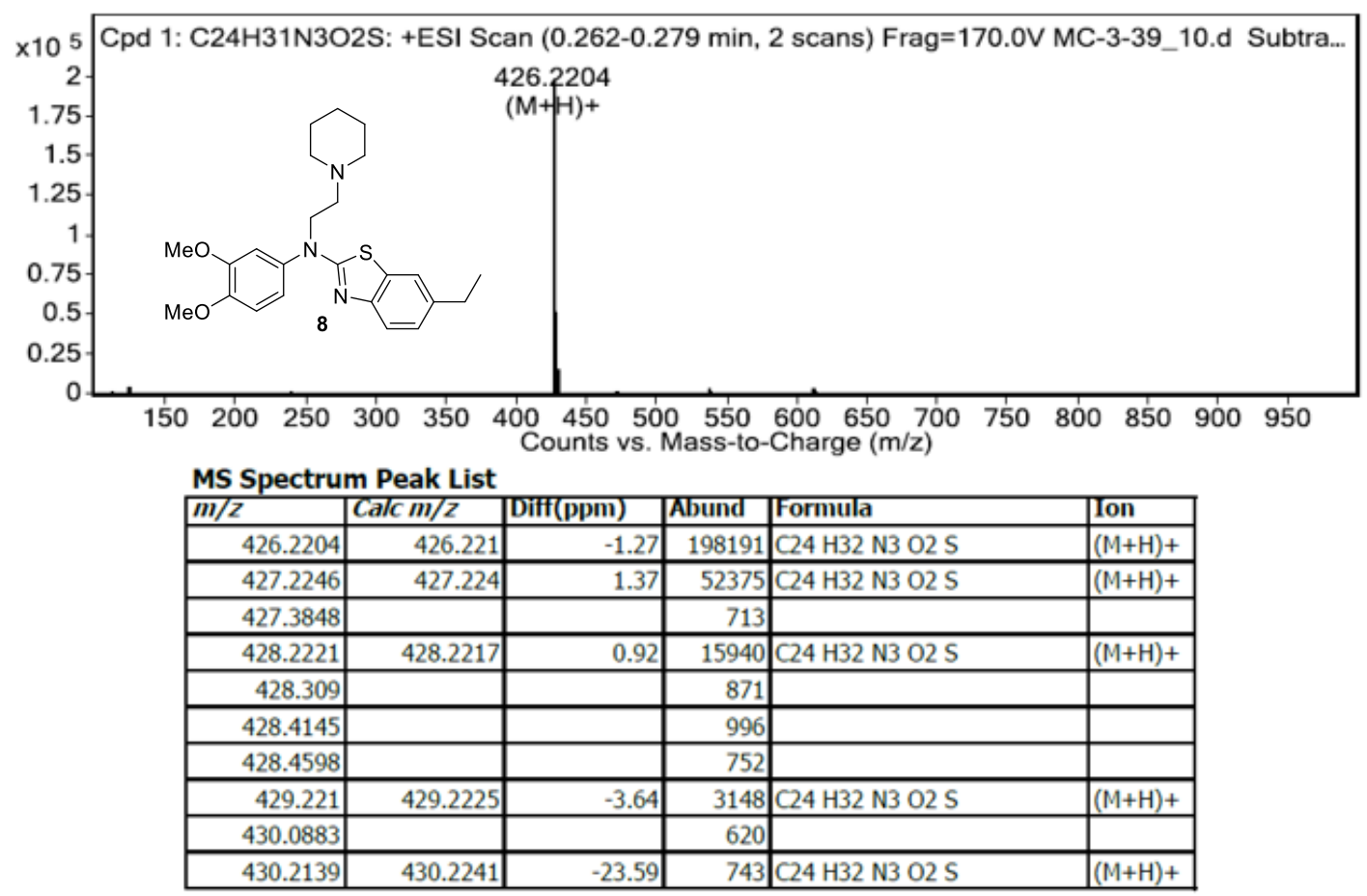

$N$-(3,4-Dimethoxyphenyl)-6-ethyl- $N$-(3-(piperidin-1-yl)propyl)benzo[d] thiazol-2-amine (9)

HRMS (ESI): calcd. for $\mathrm{C}_{25} \mathrm{H}_{34} \mathrm{~N}_{3} \mathrm{O}_{2} \mathrm{~S}[\mathrm{M}+\mathrm{H}]^{+} 440.2366$, found 440.2370 .

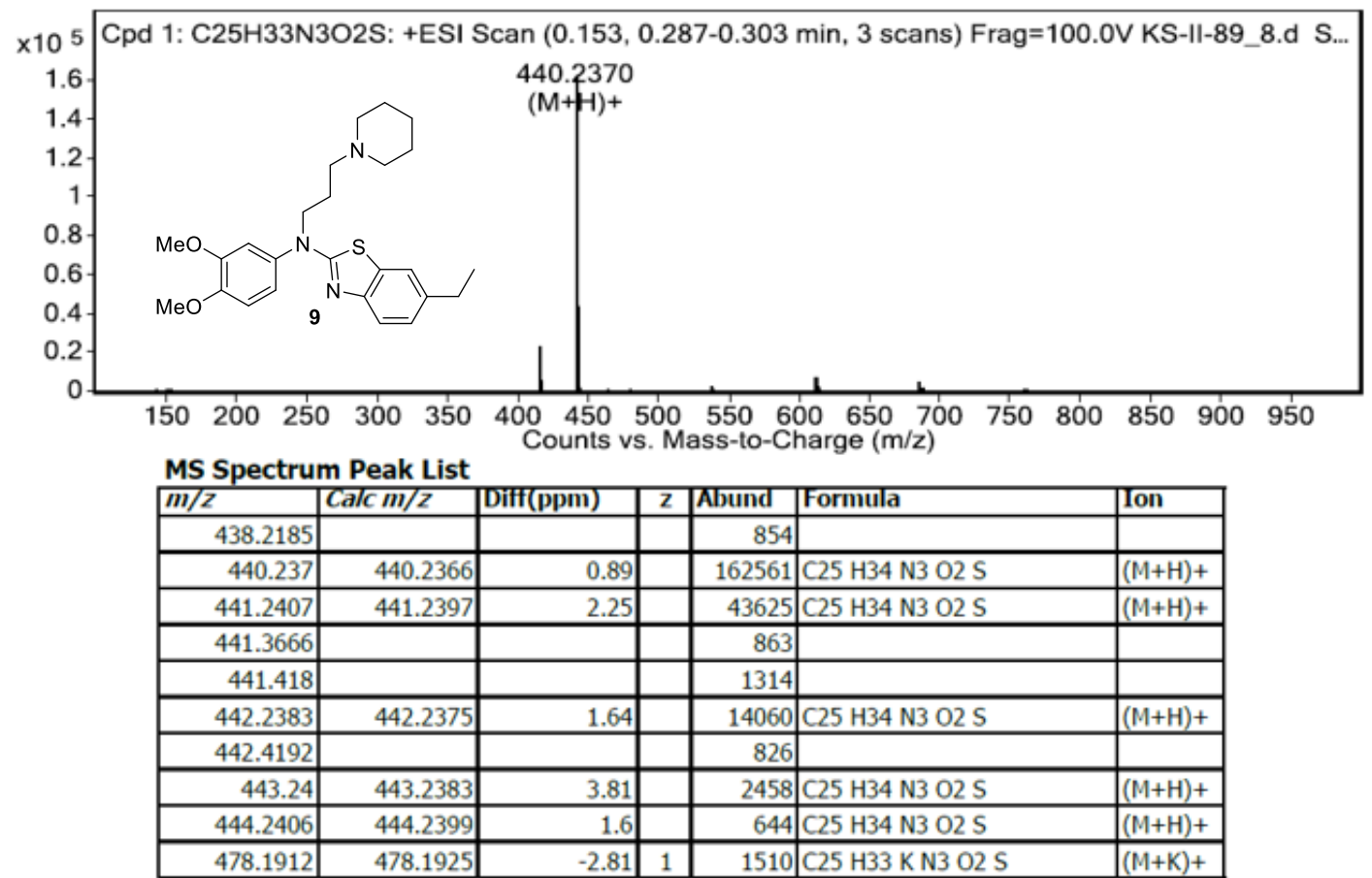


HRMS (ESI): calcd. for $\mathrm{C}_{26} \mathrm{H}_{36} \mathrm{~N}_{3} \mathrm{O}_{2} \mathrm{~S}[\mathrm{M}+\mathrm{H}]^{+} 454.2523$, found 454.2527 .

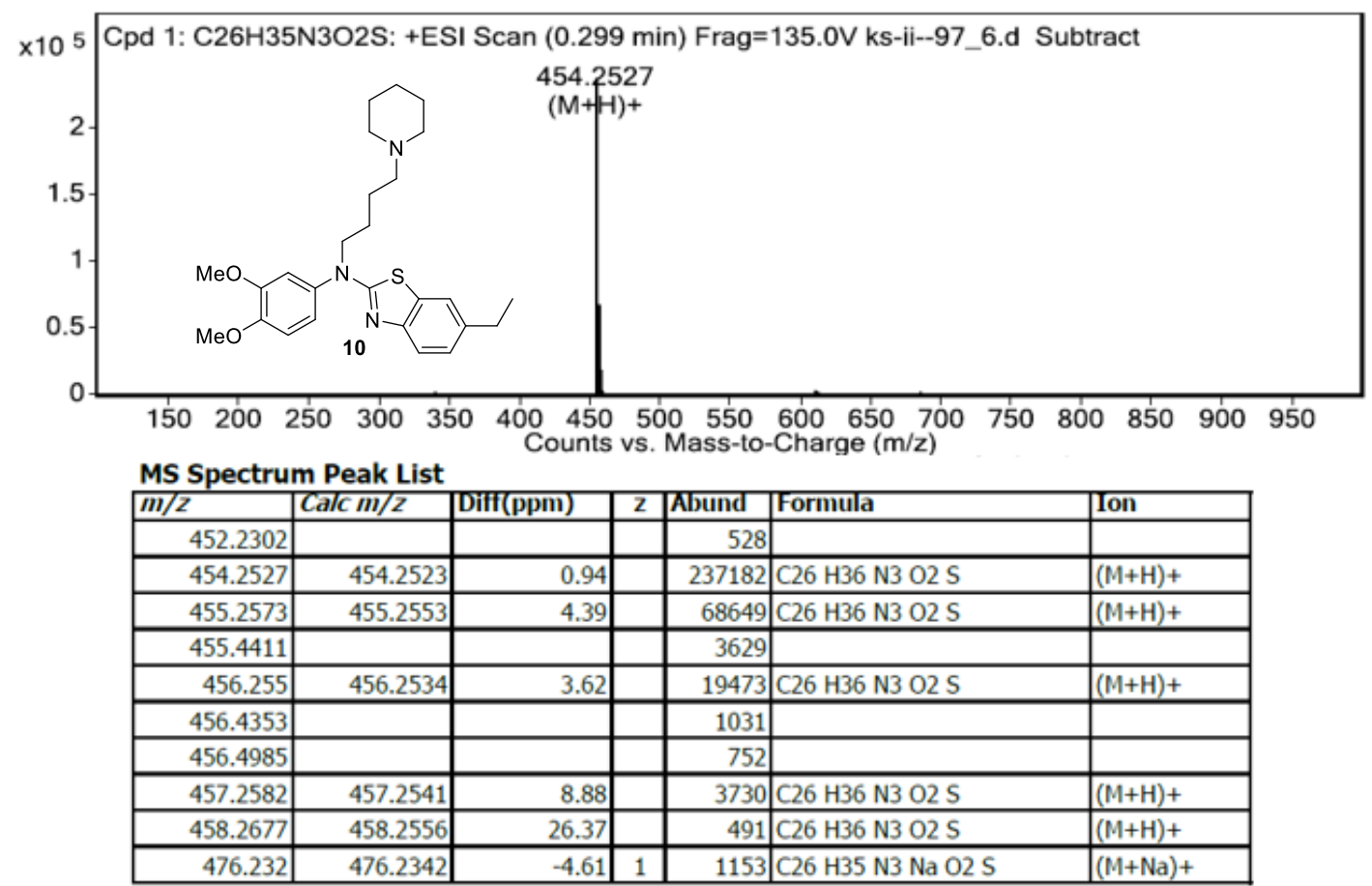


HRMS (FAB): calcd. for $\mathrm{C}_{27} \mathrm{H}_{38} \mathrm{~N}_{3} \mathrm{O}_{2} \mathrm{~S}[\mathrm{M}+\mathrm{H}]^{+}$468.2685, found 468.2690.
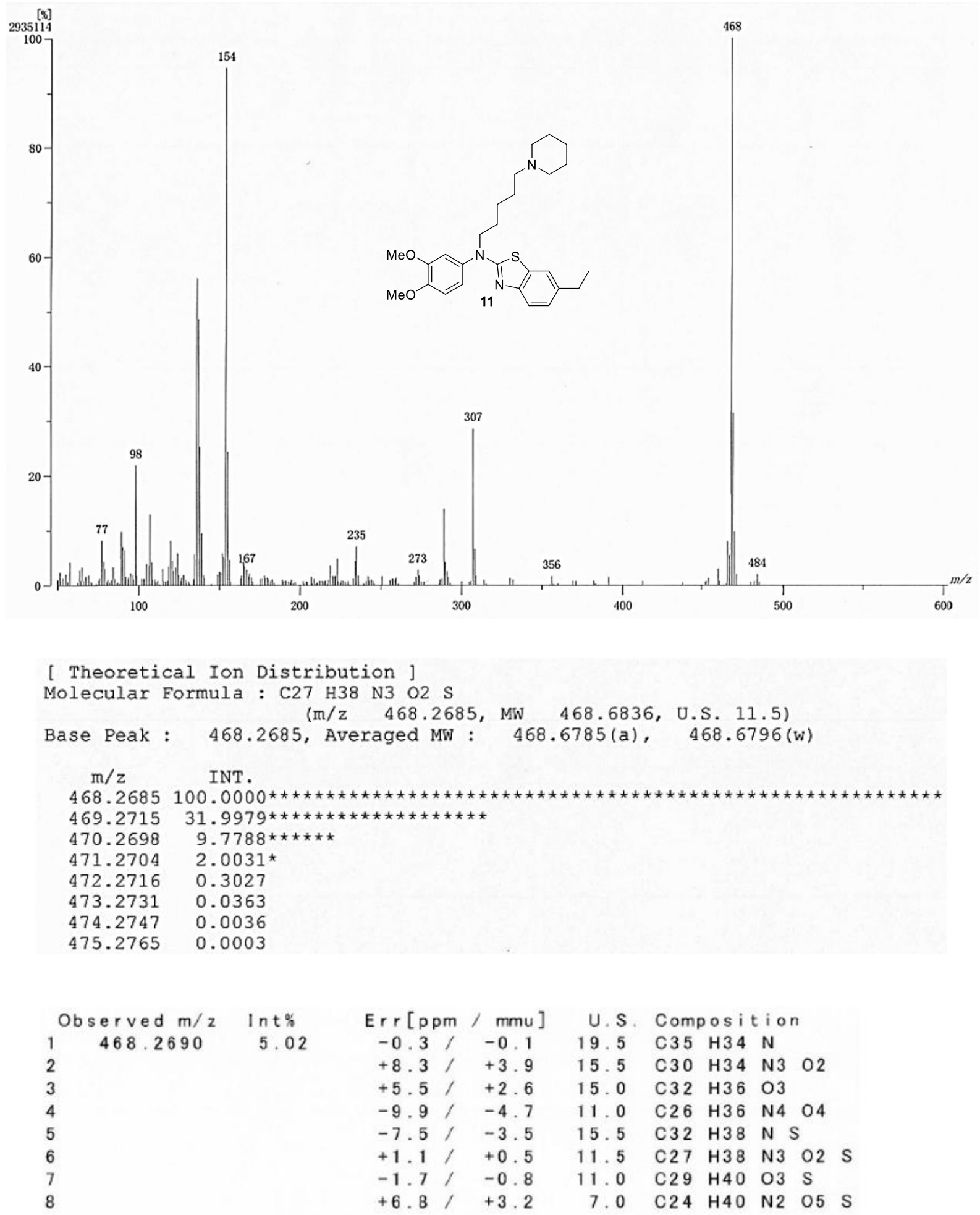

\begin{tabular}{|c|c|c|c|c|c|}
\hline $\operatorname{Err}[p p m$ & / $\mathrm{mmu}]$ & U.S. & Com & $0 \mathrm{sit}$ & ion \\
\hline$-0.3 /$ & -0.1 & 19.5 & C35 & H3 4 & $N$ \\
\hline$+8.3 /$ & +3.9 & 15.5 & C 30 & H34 & N3 02 \\
\hline$+5.5 /$ & +2.6 & 15.0 & C 32 & H3 6 & 03 \\
\hline$-9.9 /$ & -4.7 & 11.0 & C 26 & H3 6 & $\mathrm{~N} 4 \quad 04$ \\
\hline$-7.5 /$ & -3.5 & 15.5 & C32 & H3 8 & $\mathrm{NS}$ \\
\hline$+1.1 /$ & +0.5 & 11.5 & C 27 & $\mathrm{H} 38$ & N3 $\mathrm{O}_{2}$ \\
\hline$-1.7 /$ & -0.8 & 11.0 & C 29 & $\mathrm{H} 40$ & $03 \mathrm{~s}$ \\
\hline+6.8 & +3.2 & 7.0 & C24 & $\mathrm{H} 40$ & N2 05 \\
\hline
\end{tabular}


HRMS (FAB): calcd. for $\mathrm{C}_{24} \mathrm{H}_{32} \mathrm{~N}_{3} \mathrm{OS}[\mathrm{M}+\mathrm{H}]^{+} 410.2266$, found 410.2260 .

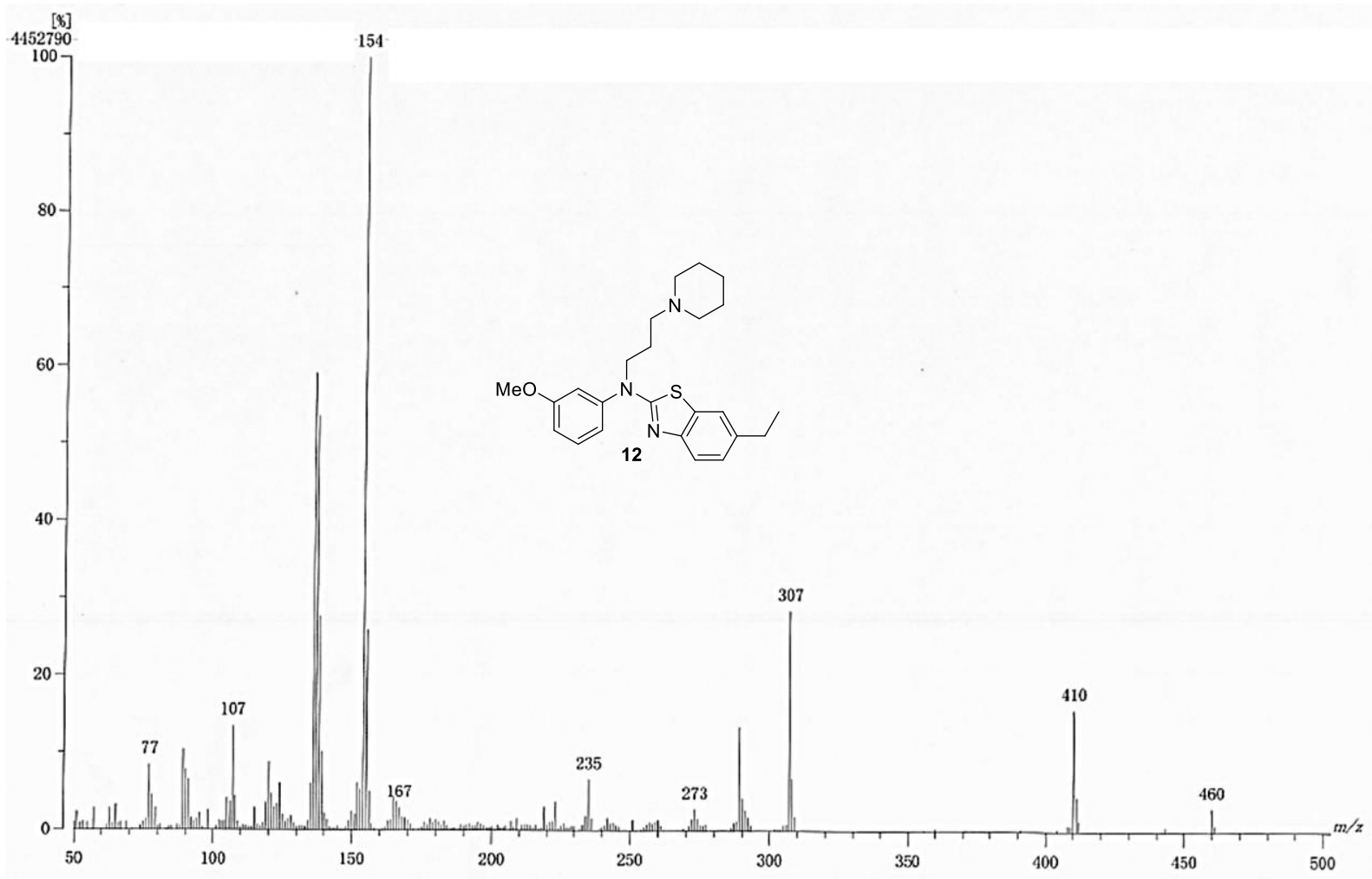

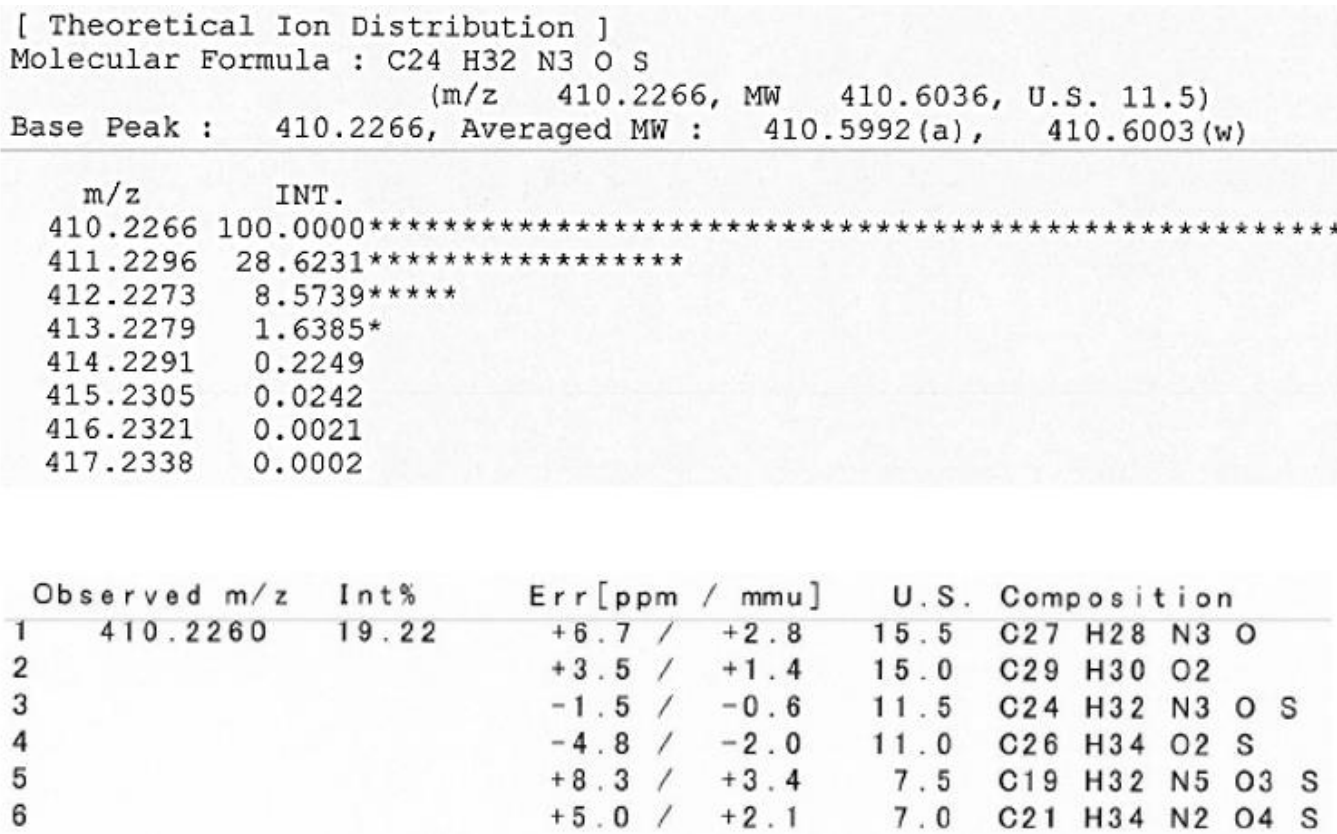


HRMS (FAB): calcd. for $\mathrm{C}_{23} \mathrm{H}_{28} \mathrm{~F}_{2} \mathrm{~N}_{3} \mathrm{~S}[\mathrm{M}+\mathrm{H}]^{+}$416.1972, found 416.1964 .

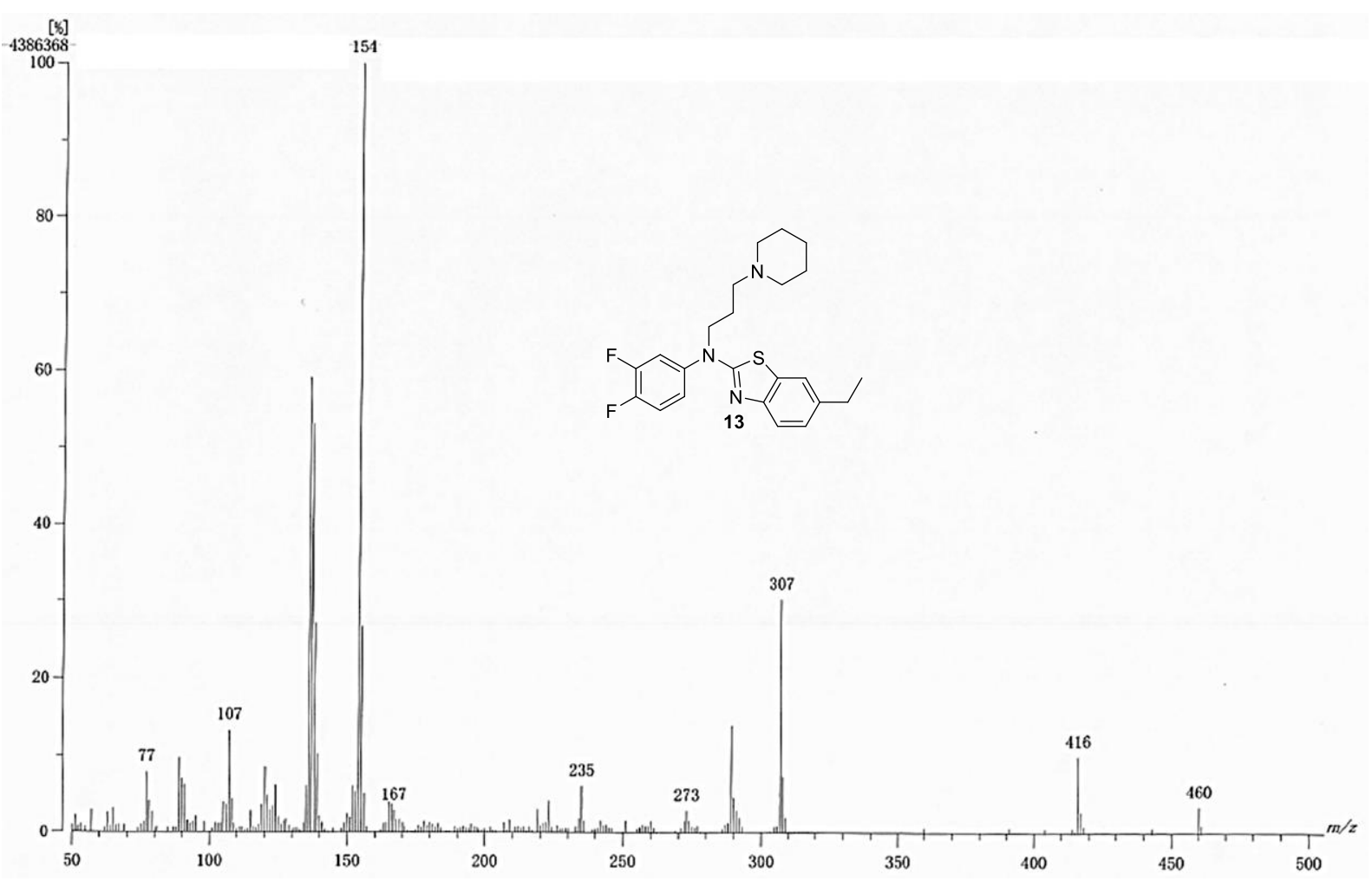

[ Theoretical Ion Distribution ]

Molecular Formula : C23 H28 F2 N3 s

(m/z 416.1972, MW 416.5582, U.S. 11.5)

Base Peak : 416.1972, Averaged Mw : 416.5543 (a), 416.5554 (w)

$\mathrm{m} / \mathrm{z} \quad \mathrm{INT}$

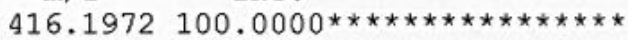

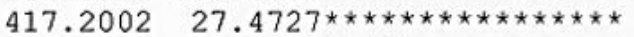

$418.1976 \quad 8.0570 * \star \star \star \star *$

419.19821 .4884 *

$420.1994 \quad 0.1910$

$421.2006 \quad 0.0188$

$422.2021 \quad 0.0015$

\begin{tabular}{lllrlllll}
\multicolumn{2}{c}{ Observed m/z } & Int\% & Err[ppm / mmu $]$ & \multicolumn{2}{c}{ U.S. Composition } \\
1 & 416.1964 & 70.17 & $+5.7 /$ & +2.4 & 19.0 & C31 H25 F \\
2 & & & $+6.2 /$ & +2.6 & 15.5 & C26 H24 F2 N3 \\
3 & & & $-8.9 /$ & -3.7 & 19.0 & C28 H24 N4 & \\
4 & & & $-2.4 /$ & -1.0 & 15.0 & C28 H29 F S & \\
5 & & & $-1.9 /$ & -0.8 & 11.5 & C23 H28 F2 N3 S
\end{tabular}


HRMS (FAB): calcd. for $\mathrm{C}_{24} \mathrm{H}_{29} \mathrm{~F}_{3} \mathrm{~N}_{3} \mathrm{~S}[\mathrm{M}+\mathrm{H}]^{+}$448.2034, found 448.2024.

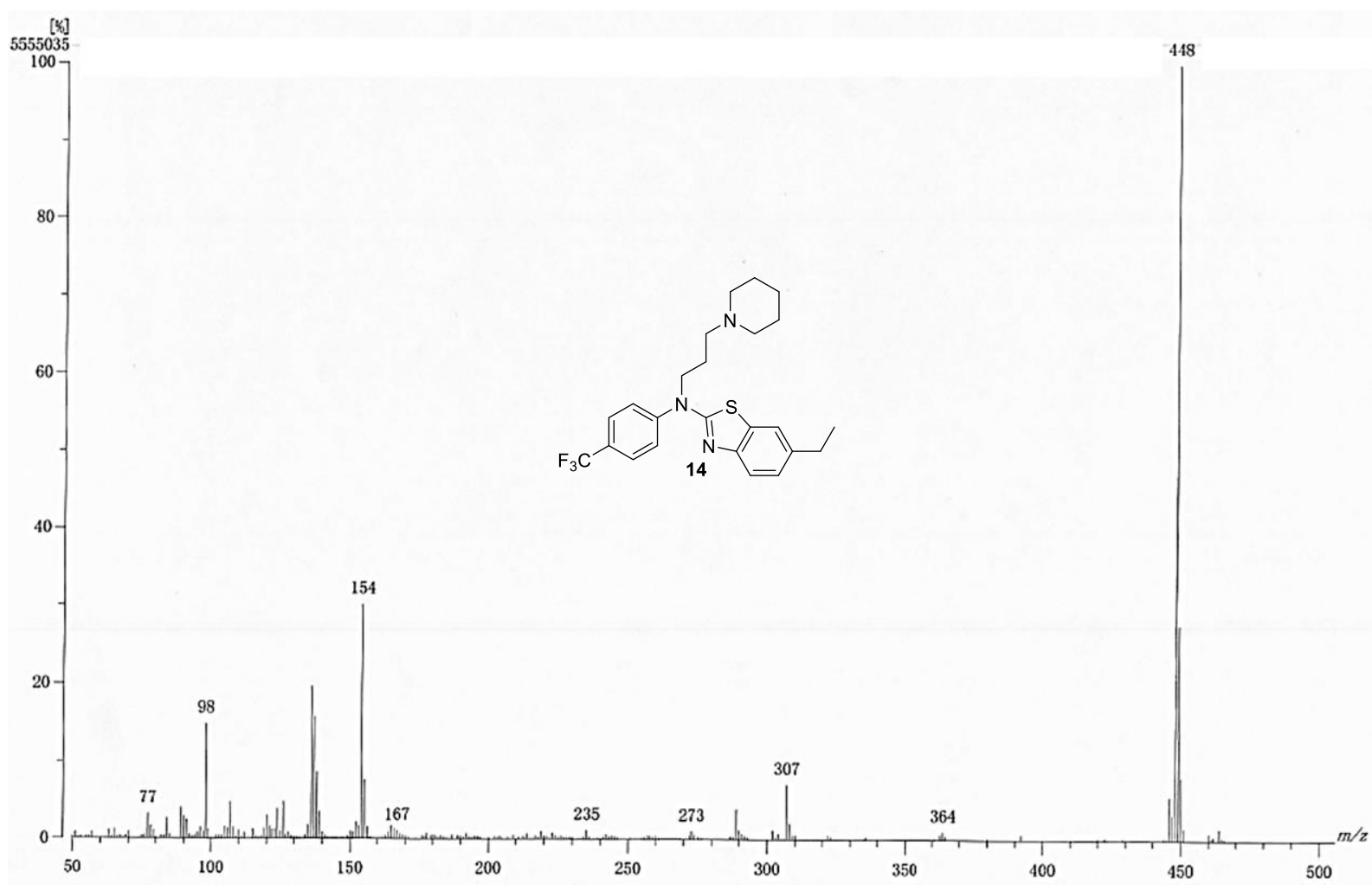

[ Theoretical Ion Distribution ]

Molecular Formula : C24 H29 F3 N3 S

$(\mathrm{m} / \mathrm{z} \quad 448.2034$, MW 448.5756 , U.S. 11.5)

Base Peak : 448.2034, Averaged MW : 448.5716 (a), 448.5726 (w)

$\mathrm{m} / \mathrm{z} \quad$ INT.

$448.2034100 .0000^{*} * *$

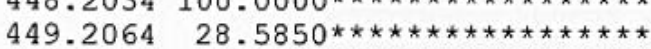

$450.2040 \quad 8.3626 * * \star * *$

451.20451 .5780 *

$452.2058 \quad 0.2075$

$453.2071 \quad 0.0210$

$454.2086 \quad 0.0017$

$455.2103 \quad 0.0001$

$\begin{array}{ccccccl}\text { Observed m/z } & \text { Int\% } & \text { Err[ppm / mmu } & \text { U.S. Composition } \\ 1 & 448.2024 & 8.20 & +4.8 / & +2.1 & 19.0 & \text { C32 H26 F2 } \\ 2 & & & +5.2 / & +2.3 & 15.5 & \text { C27 H25 F3 N3 } \\ 3 & & & -8.8 / & -3.9 & 19.0 & \text { C29 H25 F N4 } \\ 4 & & -2.7 / & -1.2 & 15.0 & \text { C29 H30 F2 S } \\ 5 & & -2.3 / & -1.0 & 11.5 & \text { C24 H29 F3 N3 S }\end{array}$


$N$-(3,4-Dimethoxyphenyl)-6-ethyl- $N$-(2-(pyrrolidin-1-yl)ethyl)benzo[d] thiazol-2-amine (15)

HRMS (ESI): calcd. for $\mathrm{C}_{23} \mathrm{H}_{30} \mathrm{~N}_{3} \mathrm{O}_{2} \mathrm{~S}[\mathrm{M}+\mathrm{H}]^{+}$412.2053, found 412.2048 .

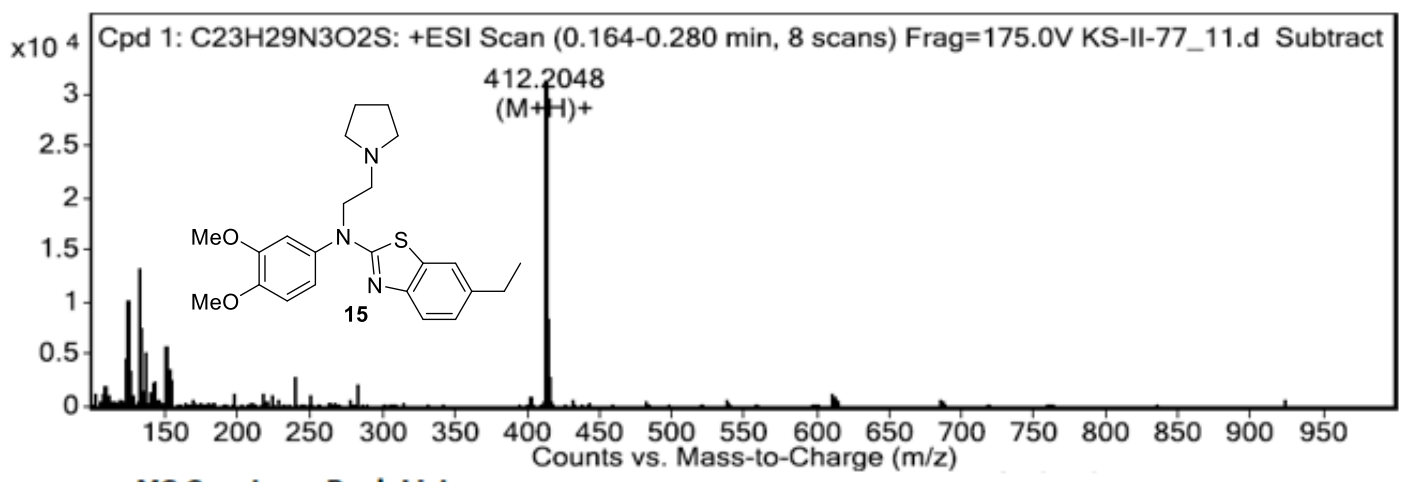

MS Spectrum Peak List

\begin{tabular}{|r|r|r|r|r|l|l|}
\hline $\boldsymbol{m} / \boldsymbol{z}$ & $C a / c m / z$ & Diff(ppm) & Z & Abund & Formula & Ion \\
\hline 411.1954 & & & & 638 & & \\
\hline 412.2048 & 412.2053 & -1.39 & & 31293 & $\mathrm{C} 23 \mathrm{H} 30 \mathrm{~N} 3 \mathrm{O} 2 \mathrm{~S}$ & $(\mathrm{M}+\mathrm{H})+$ \\
\hline 412.341 & & & & 897 & & \\
\hline 412.3818 & & & & 889 & & \\
\hline 412.4355 & & & & 552 & & \\
\hline 413.2095 & 413.2083 & 2.68 & & 8460 & $\mathrm{C} 23 \mathrm{H} 30 \mathrm{~N} 3 \mathrm{O} 2 \mathrm{~S}$ & $(\mathrm{M}+\mathrm{H})+$ \\
\hline 413.3877 & & & & 454 & & \\
\hline 414.2085 & 414.2058 & 6.46 & & 3053 & $\mathrm{C} 23 \mathrm{H} 30 \mathrm{~N} 3 \mathrm{O} 2 \mathrm{~S}$ & $(\mathrm{M}+\mathrm{H})+$ \\
\hline 415.2085 & 415.2067 & 4.29 & & 666 & $\mathrm{C} 23 \mathrm{H} 30 \mathrm{~N} 3 \mathrm{O} 2 \mathrm{~S}$ & $(\mathrm{M}+\mathrm{H})+$ \\
\hline 434.1883 & 434.1873 & 2.28 & 1 & 234 & $\mathrm{C} 23 \mathrm{H} 29 \mathrm{~N} 3 \mathrm{Na} \mathrm{O} 2 \mathrm{~S}$ & $(\mathrm{M}+\mathrm{Na})+$ \\
\hline
\end{tabular}


HRMS (FAB): calcd. for $\mathrm{C}_{24} \mathrm{H}_{32} \mathrm{~N}_{3} \mathrm{O}_{2} \mathrm{~S}[\mathrm{M}+\mathrm{H}]^{+} 426.2215$, found 426.2220 .

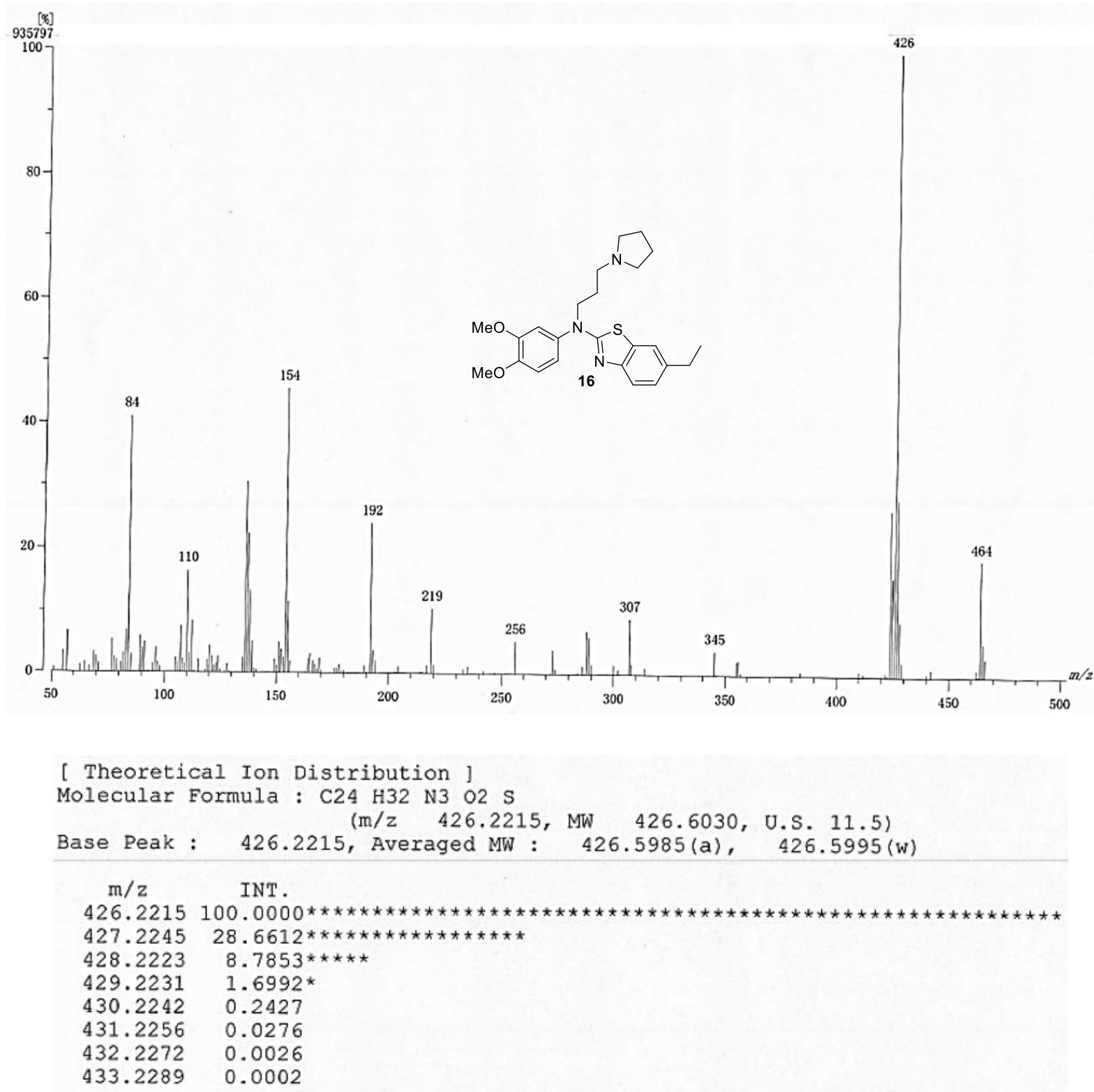

\begin{tabular}{|c|c|c|c|c|c|c|c|c|c|}
\hline & erved $\mathrm{m} / \mathrm{z}$ & Int $\%$ & $\operatorname{Err}[\mathrm{pp}$ & / $\mathrm{mmu}]$ & U.S. & \multicolumn{4}{|c|}{ Composition } \\
\hline 1 & 426.2220 & 54.75 & -0.4 & -0.2 & 19.5 & C 32 & $\mathrm{H} 28$ & $\mathrm{~N}$ & \\
\hline 2 & & & +9.0 & +3.8 & 15.5 & $\mathrm{C} 27$ & $\mathrm{H} 28$ & N3 & O2 \\
\hline 3 & & & +5.9 & +2.5 & 15.0 & C29 & $\mathrm{H} 30$ & 03 & \\
\hline 4 & & & -11.0 & -4.7 & 11.0 & C23 & $\mathrm{H} 30$ & N4 & 04 \\
\hline 5 & & & -4.8 & -2.0 & 6.5 & $\mathrm{C} 20$ & $\mathrm{H} 32$ & N3 & 07 \\
\hline 6 & & & -7.9 & $-3,4$ & 6.0 & $\mathrm{C} 22$ & $\mathrm{H} 34$ & 08 & \\
\hline 7 & & & +1.5 & +0.7 & 2. 0 & C 17 & $\mathrm{H} 34$ & N2 & 010 \\
\hline 8 & & & $-8 \cdot 3$ & $-3 \cdot 5$ & 15.5 & C 29 & $\mathrm{H} 32$ & $\mathrm{~N} S$ & \\
\hline 9 & & & +1.1 & +0.5 & 11.5 & $\mathrm{C} 24$ & $\mathrm{H} 32$ & N3 & $\mathrm{O} 2$ \\
\hline 0 & & & -2.0 & -0.9 & 11.0 & $\mathrm{C} 26$ & $\mathrm{H} 34$ & 03 & S \\
\hline 1 & & & +7.4 & +3.2 & 7.0 & $\mathrm{C} 21$ & $\mathrm{H} 34$ & N2 & 05 \\
\hline
\end{tabular}


$N$-(3,4-Dimethoxyphenyl)-6-ethyl- $N$-(4-(pyrrolidin-1-yl)butyl)benzo[ $d]$ thiazol-2-amine (17)

HRMS (ESI): calcd. for $\mathrm{C}_{25} \mathrm{H}_{34} \mathrm{~N}_{3} \mathrm{O}_{2} \mathrm{~S}[\mathrm{M}+\mathrm{H}]^{+} 440.2366$, found 440.2364 .

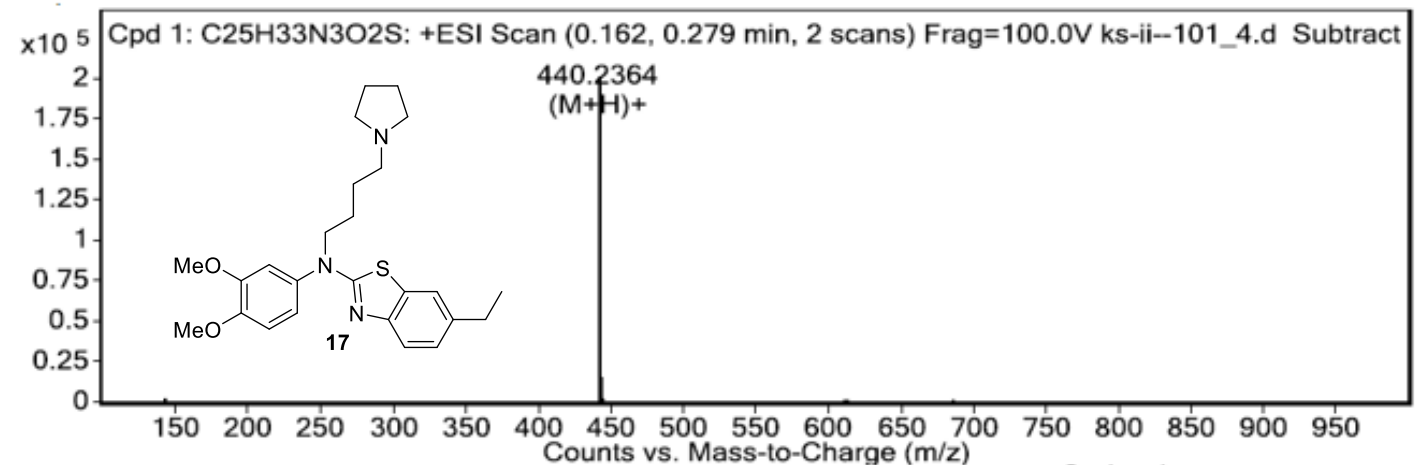

MS Spectrum Peak List

\begin{tabular}{|r|r|r|r|l|l|}
\hline$m / z$ & Calc m/z & Diff(ppm) & Abund & Formula & Ion \\
\hline 438.2152 & & & 627 & & \\
\hline 440.2364 & 440.2366 & -0.61 & 200644 & $\mathrm{C} 25 \mathrm{H} 34 \mathrm{~N} 3 \mathrm{O} 2 \mathrm{~S}$ & $(\mathrm{M}+\mathrm{H})+$ \\
\hline 441.2403 & 441.2397 & 1.43 & 52762 & $\mathrm{C} 25 \mathrm{H} 34 \mathrm{~N} 3 \mathrm{O} 2 \mathrm{~S}$ & $(\mathrm{M}+\mathrm{H})+$ \\
\hline 442.2398 & 442.2375 & 5 & 15790 & $\mathrm{C} 25 \mathrm{H} 34 \mathrm{~N} 3 \mathrm{O} 2 \mathrm{~S}$ & $(\mathrm{M}+\mathrm{H})+$ \\
\hline 442.3915 & & & 976 & & \\
\hline 442.4858 & & & 625 & & \\
\hline 443.2384 & 443.2383 & 0.28 & 3468 & $\mathrm{C} 25 \mathrm{H} 34 \mathrm{~N} 3 \mathrm{O} 2 \mathrm{~S}$ & $(\mathrm{M}+\mathrm{H})+$ \\
\hline 443.2943 & & & 1097 & & \\
\hline 443.3376 & & & 581 & & \\
\hline 444.247 & 444.2399 & 16.14 & 679 & $\mathrm{C} 25 \mathrm{H} 34 \mathrm{~N} 3 \mathrm{O} 2 \mathrm{~S}$ & $(\mathrm{M}+\mathrm{H})+$ \\
\hline
\end{tabular}


HRMS (FAB): calcd. for $\mathrm{C}_{26} \mathrm{H}_{36} \mathrm{~N}_{3} \mathrm{O}_{2} \mathrm{~S}[\mathrm{M}+\mathrm{H}]^{+} 454.2528$, found 454.2520 .

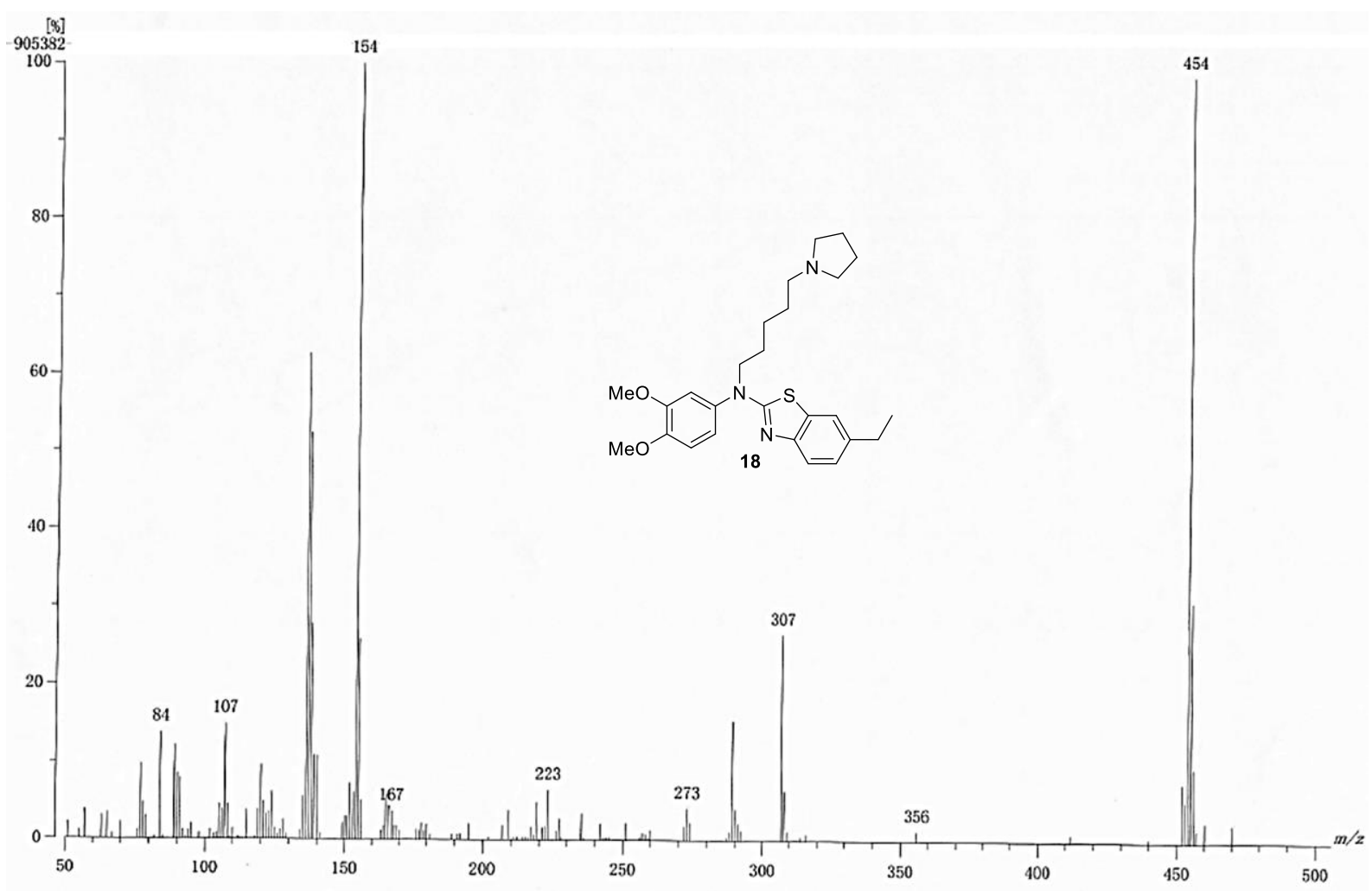

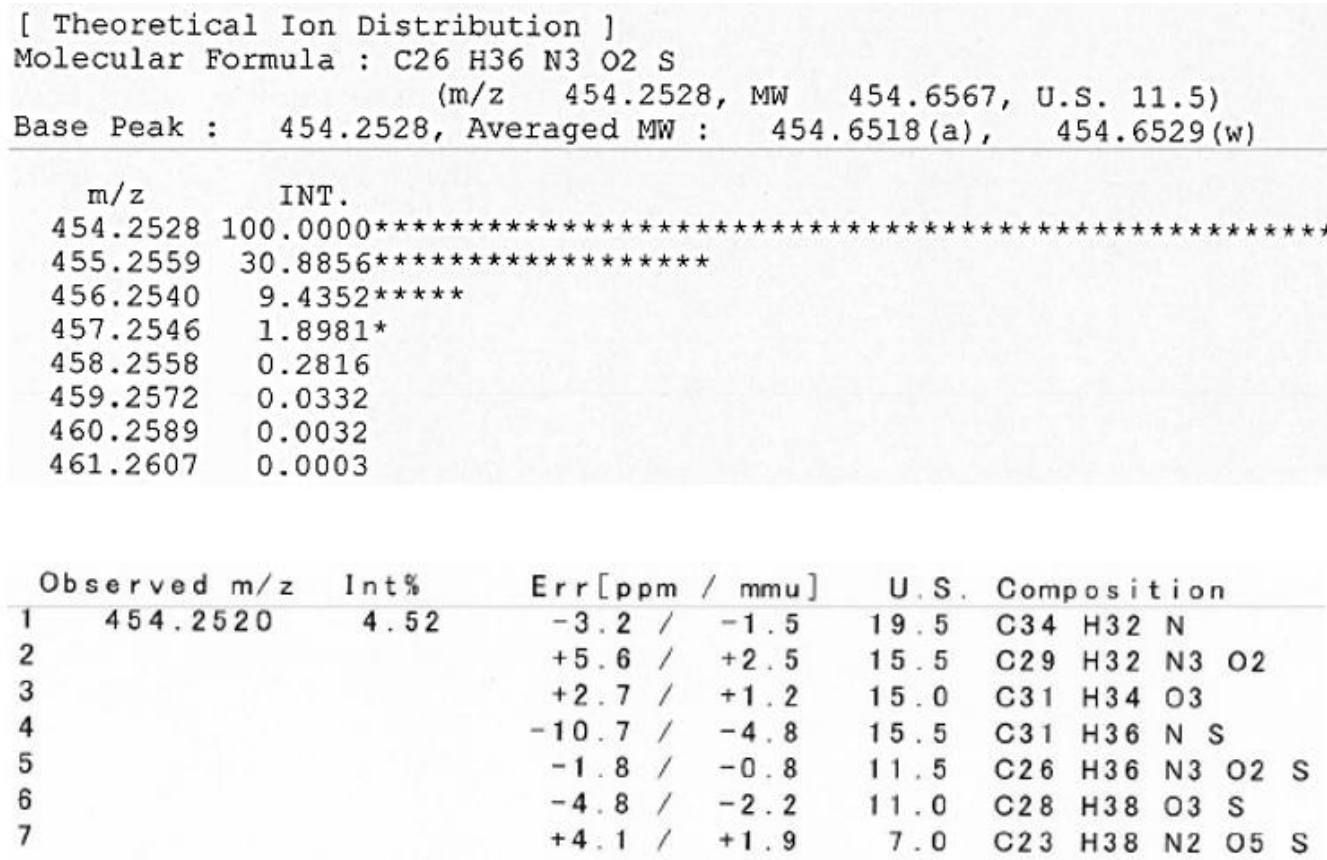




\section{$N$-(3,4-Dimethoxyphenyl)-6-ethyl- $N$-(2-morpholinoethyl)benzo[d] thiazol-2-amine (19)}

HRMS (ESI): calcd. for $\mathrm{C}_{23} \mathrm{H}_{30} \mathrm{~N}_{3} \mathrm{O}_{3} \mathrm{~S}[\mathrm{M}+\mathrm{H}]^{+}$428.2002, found 428.1996 .

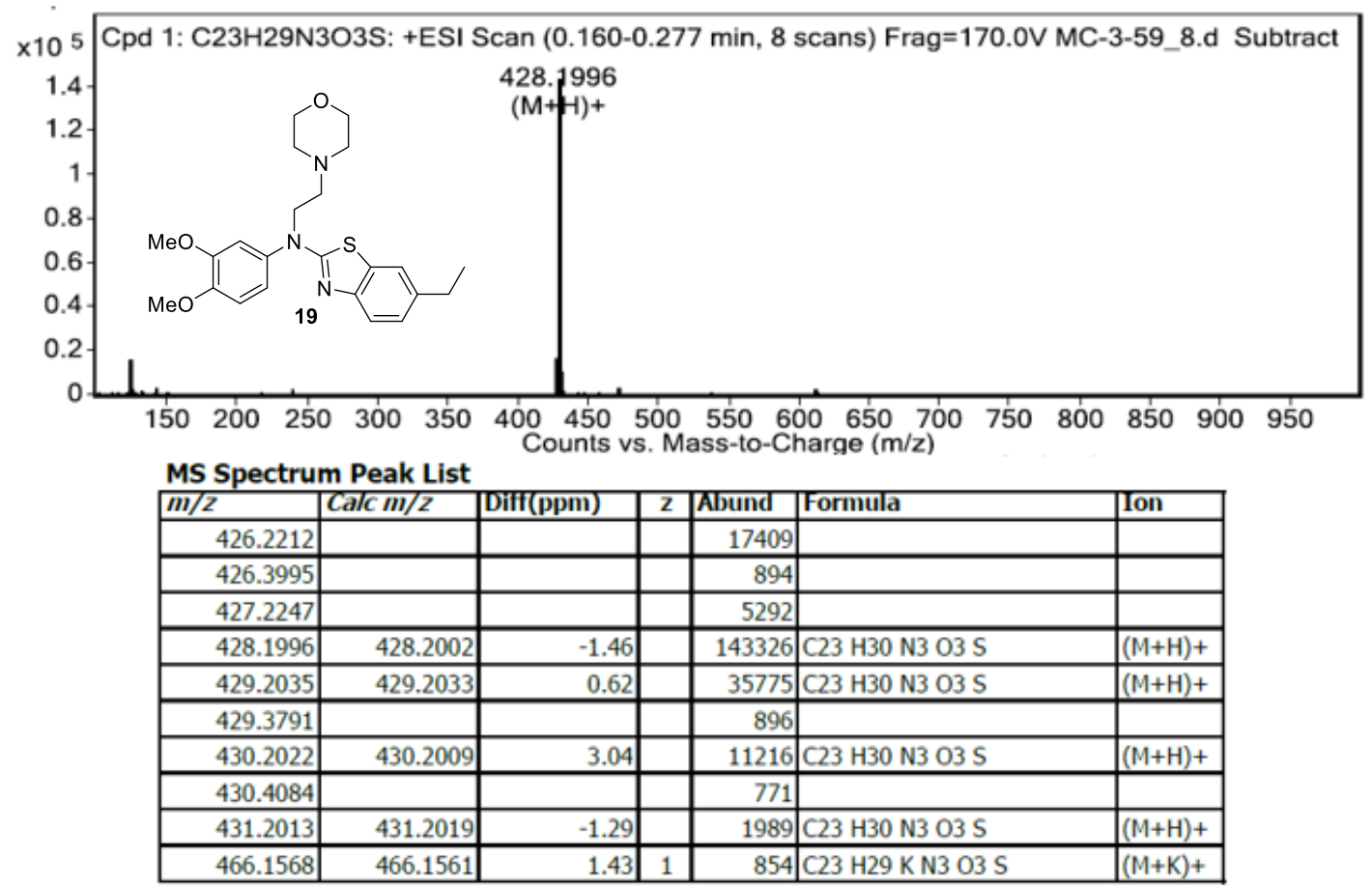

$N$-(3,4-Dimethoxyphenyl)-6-ethyl- $N$-(3-morpholinopropyl)benzo[d] thiazol-2-amine (20)

HRMS (ESI): calcd. for $\mathrm{C}_{24} \mathrm{H}_{32} \mathrm{~N}_{3} \mathrm{O}_{3} \mathrm{~S}[\mathrm{M}+\mathrm{H}]^{+} 442.2159$, found 442.2162 .

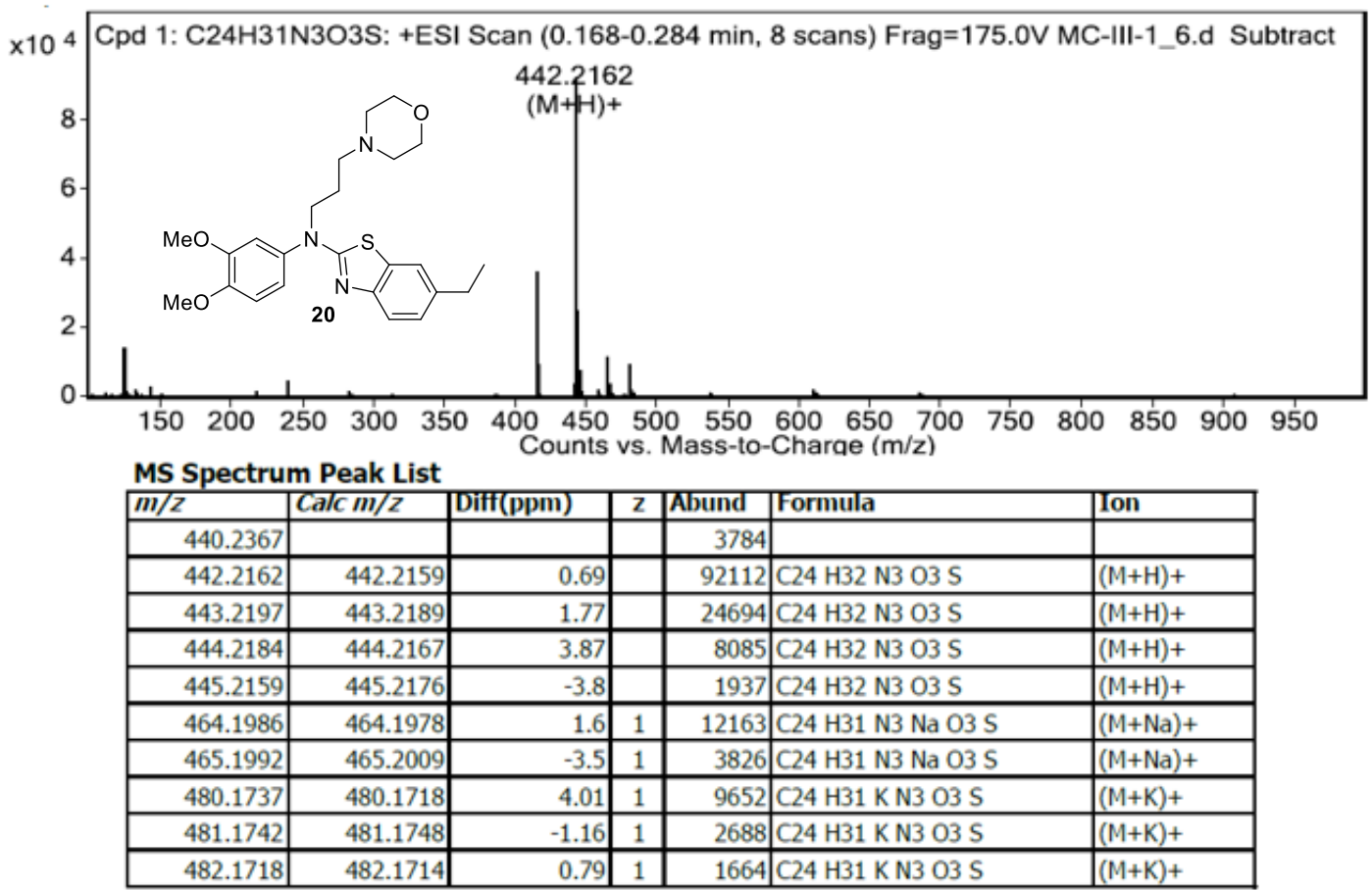


HRMS (FAB): calcd. for $\mathrm{C}_{25} \mathrm{H}_{34} \mathrm{~N}_{3} \mathrm{O}_{3} \mathrm{~S}[\mathrm{M}+\mathrm{H}]^{+}$456.2321, found 456.2313 .
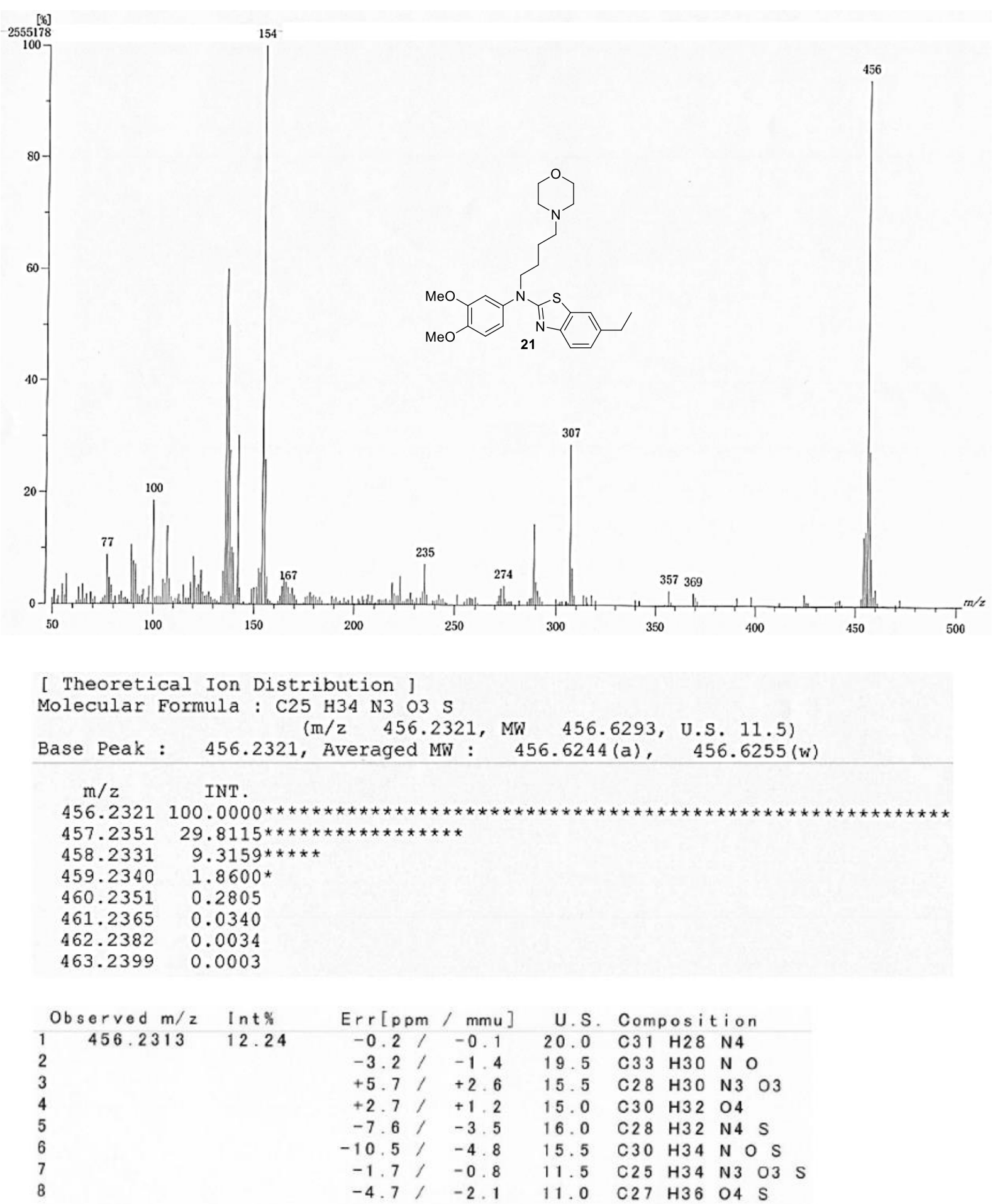

\begin{tabular}{|c|c|c|c|c|c|c|c|}
\hline Observed $\mathrm{m} / \mathrm{z}$ & $\ln t \%$ & Err $[p p r$ & $/ \mathrm{mmu}]$ & U.S & Com & os i & tion \\
\hline 456.2313 & 12.24 & -0.2 & -0.1 & 20.0 & C 31 & $\mathrm{H} 28$ & N4 \\
\hline & & -3.2 & -1.4 & 19.5 & C 33 & $\mathrm{H} 3 \mathrm{O}$ & $\mathrm{NO}$ \\
\hline & & +5.7 & +2.6 & 15.5 & C2 8 & $\mathrm{H} 3 \mathrm{O}$ & $\mathrm{N} 3 \mathrm{O} 3$ \\
\hline & & +2.7 & +1.2 & 15.0 & C30 & H3 2 & 04 \\
\hline & & -7.6 & $-3 \cdot 5$ & 16.0 & C28 & H32 & N4 S \\
\hline & & -10.5 & -4.8 & 15.5 & C 30 & H3 4 & $N O S$ \\
\hline & & -1.7 & -0.8 & 11.5 & C 25 & H3 4 & $\mathrm{~N} 3 \mathrm{O}_{3}$ \\
\hline & & -4.7 & $-2 \cdot 1$ & 11.0 & C2 27 & H3 6 & $04 \mathrm{~s}$ \\
\hline
\end{tabular}


$N$-(3,4-Dimethoxyphenyl)-6-ethyl- $N$-(5-morpholinopentyl)benzo[ $d]$ thiazol-2-amine (22)

HRMS (ESI): calcd. for $\mathrm{C}_{26} \mathrm{H}_{36} \mathrm{~N}_{3} \mathrm{O}_{3} \mathrm{~S}[\mathrm{M}+\mathrm{H}]^{+}$470.2472, found 470.2464 .

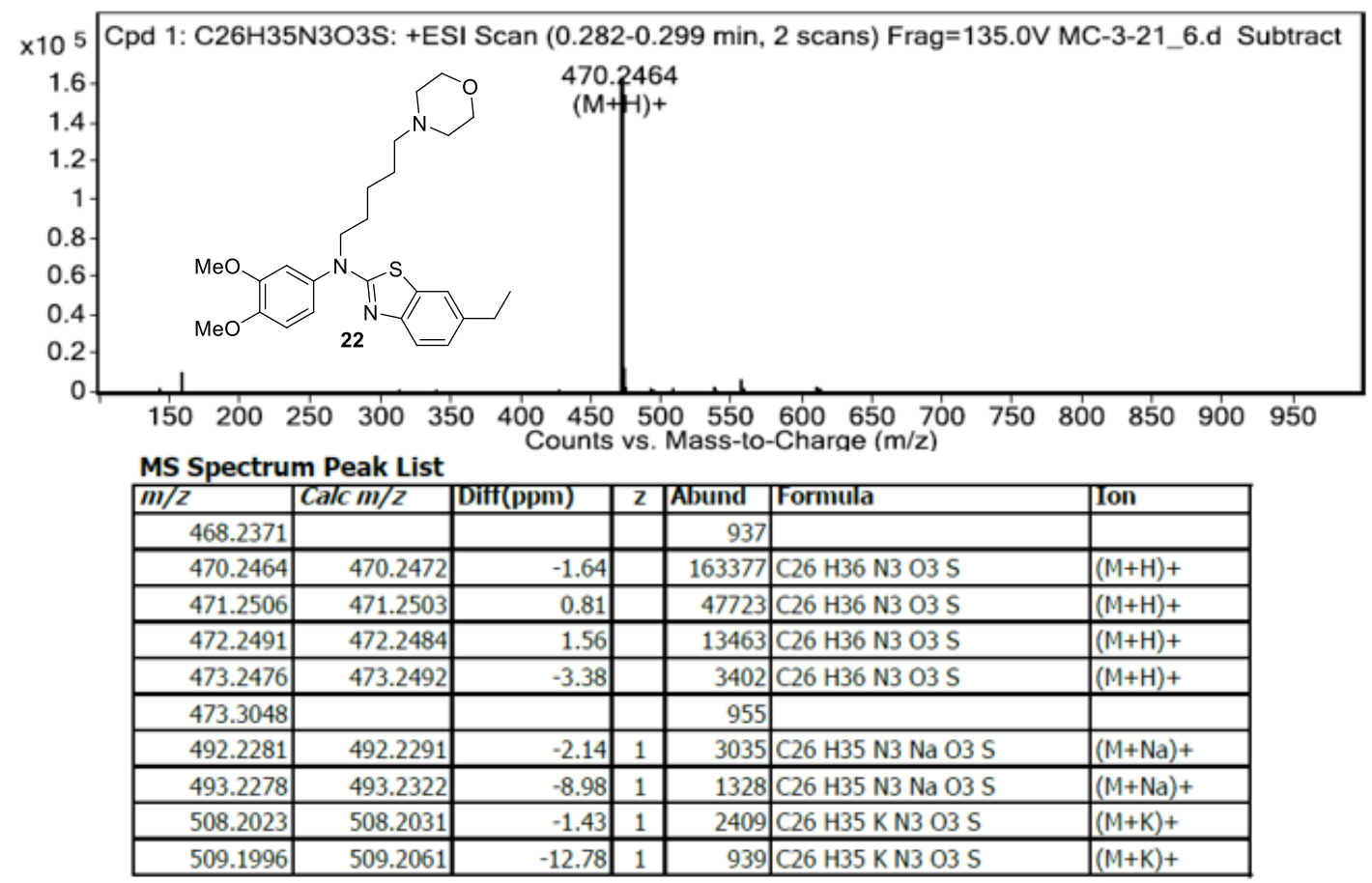

$N^{I}$-(3,4-Dimethoxyphenyl)- $N^{2}, N^{2}$-diethyl- $N^{I}$-(6-ethylbenzo[ $\left.d\right]$ thiazol-2-yl)ethane-1,2-diamine (23)

HRMS (ESI): calcd. for $\mathrm{C}_{23} \mathrm{H}_{32} \mathrm{~N}_{3} \mathrm{O}_{2} \mathrm{~S}[\mathrm{M}+\mathrm{H}]^{+}$414.2210, found 414.2204.

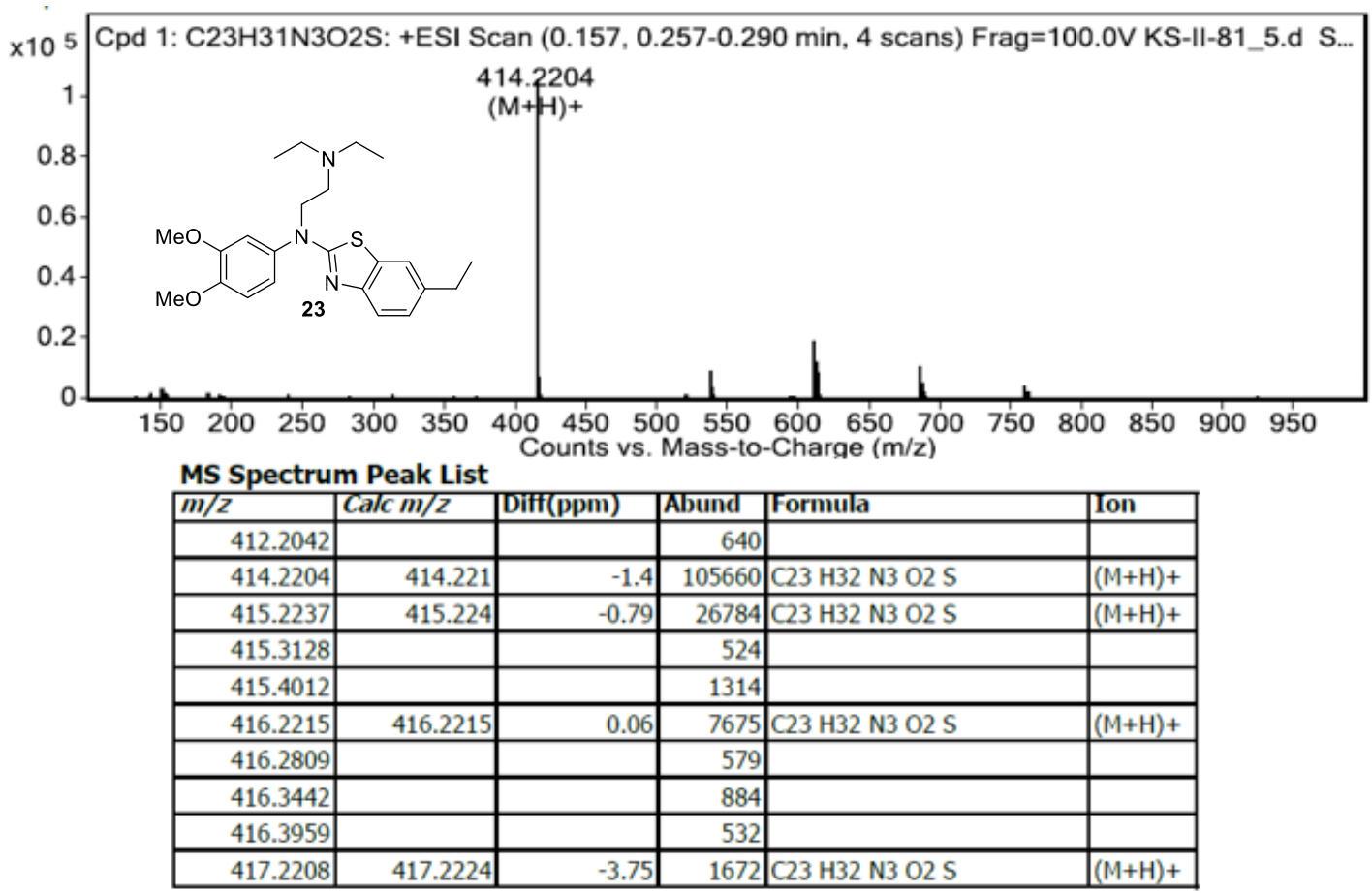


$N^{1}$-(3,4-Dimethoxyphenyl)- $N^{3}, N^{3}$-diethyl- $N^{l}$-(6-ethylbenzo[d] thiazol-2-yl)propane-1,3-diamine (24)

HRMS (ESI): calcd. for $\mathrm{C}_{24} \mathrm{H}_{34} \mathrm{~N}_{3} \mathrm{O}_{2} \mathrm{~S}[\mathrm{M}+\mathrm{H}]^{+} 428.2366$, found 428.2369 .

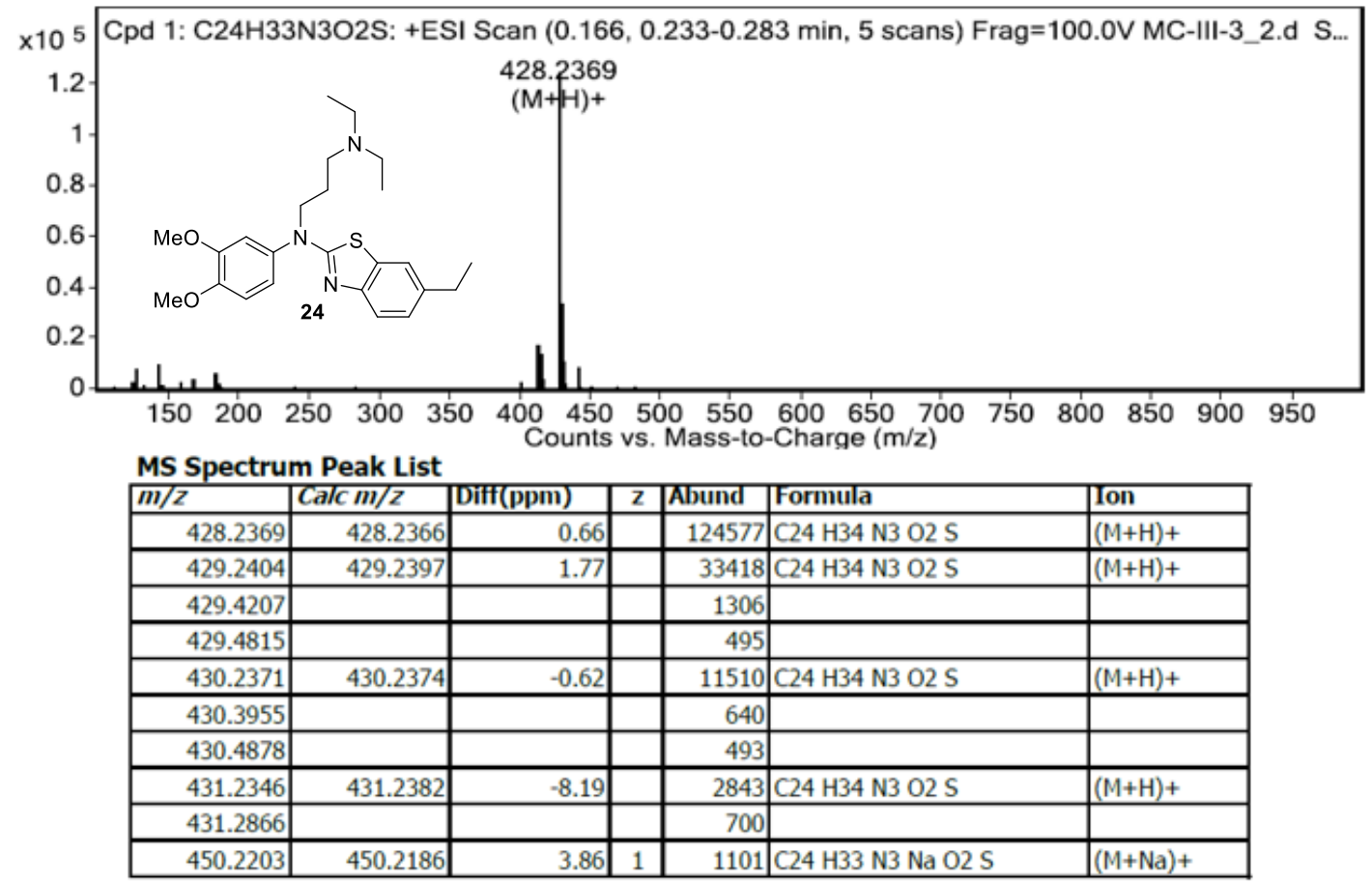

$N^{1}$-(3,4-Dimethoxyphenyl)- $N^{4}, N^{4}$-diethyl- $N^{1}$-(6-ethylbenzo[d] thiazol-2-yl)butane-1,4-diamine (25) HRMS (ESI): calcd. for $\mathrm{C}_{25} \mathrm{H}_{36} \mathrm{~N}_{3} \mathrm{O}_{2} \mathrm{~S}[\mathrm{M}+\mathrm{H}]^{+} 442.2523$, found 442.2519 .

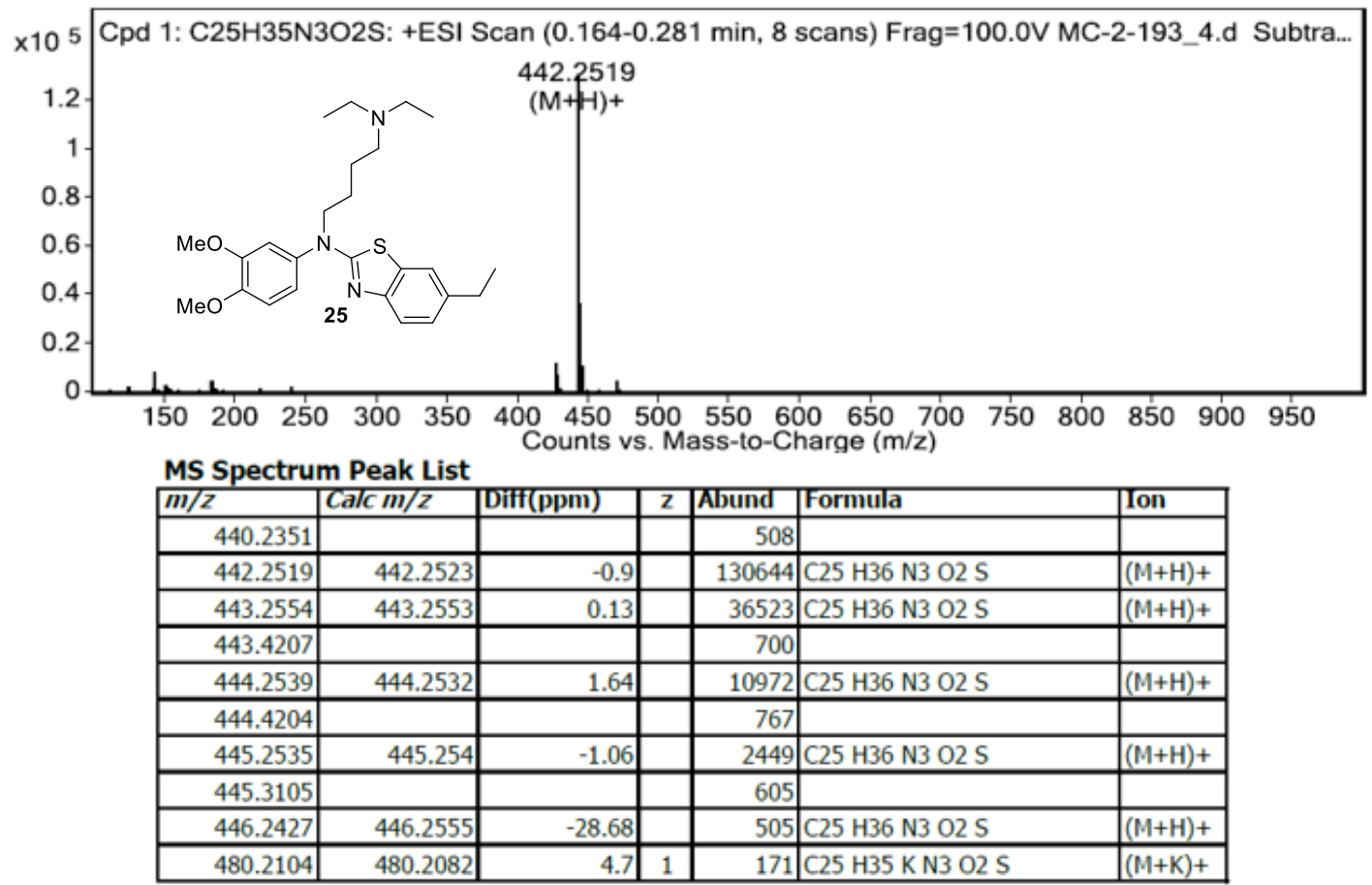


$N^{1}$-(3,4-Dimethoxyphenyl)- $N^{5}, N^{5}$-diethyl- $N^{1}$-(6-ethylbenzo $[d]$ thiazol-2-yl)pentane-1,5-diamine (26)

HRMS (ESI): calcd. for $\mathrm{C}_{26} \mathrm{H}_{38} \mathrm{~N}_{3} \mathrm{O}_{2} \mathrm{~S}[\mathrm{M}+\mathrm{H}]^{+}$456.2679, found 456.2675 .

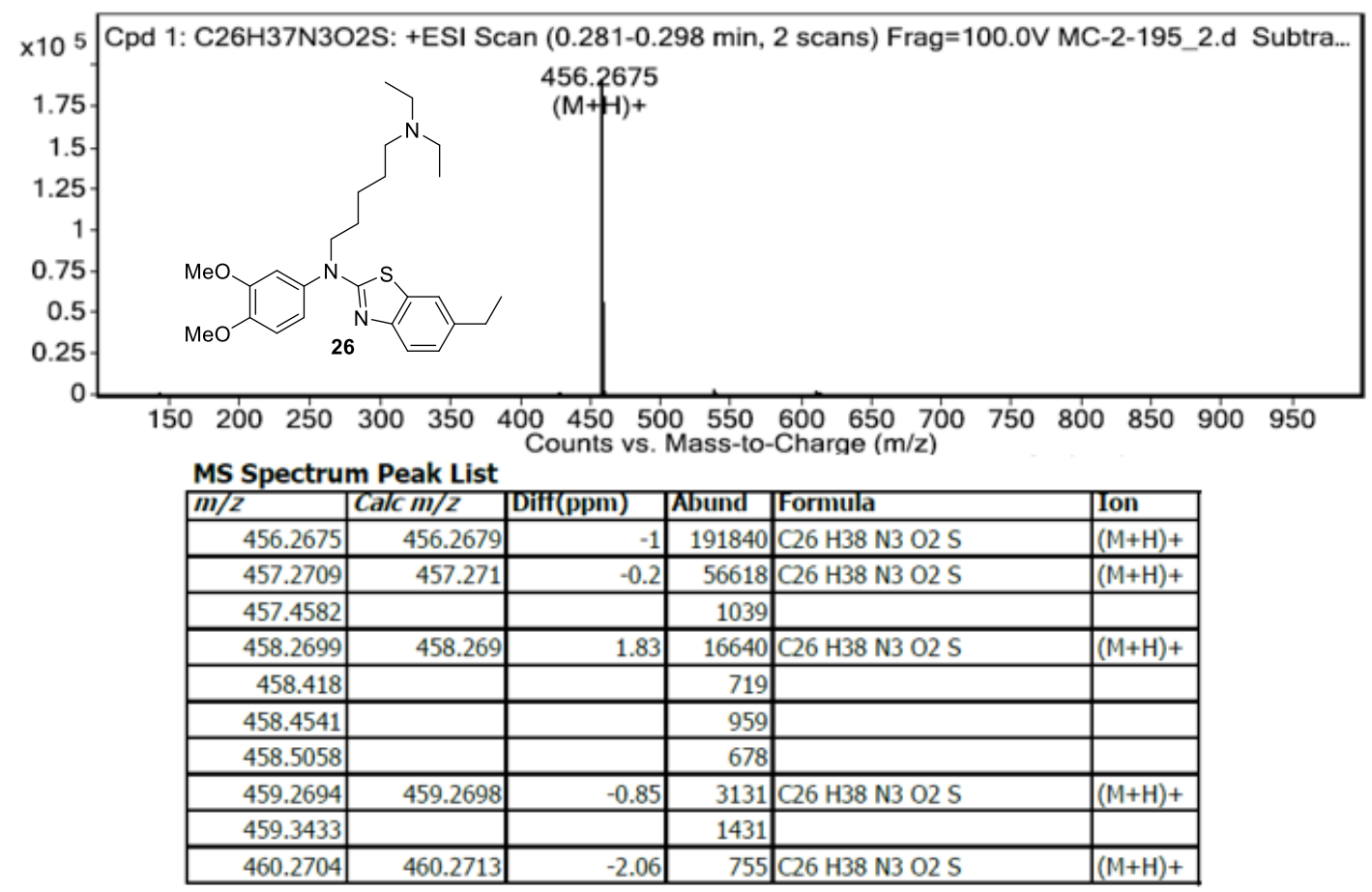

$N^{l}$-Cyclohexyl- $N^{2}$-(3,4-dimethoxyphenyl)- $N^{2}$-(6-ethylbenzo[d] thiazol-2-yl)ethane-1,2-diamine (27)

HRMS (ESI): calcd. for $\mathrm{C}_{25} \mathrm{H}_{34} \mathrm{~N}_{3} \mathrm{O}_{2} \mathrm{~S}[\mathrm{M}+\mathrm{H}]^{+} 440.2366$, found 440.2362 .

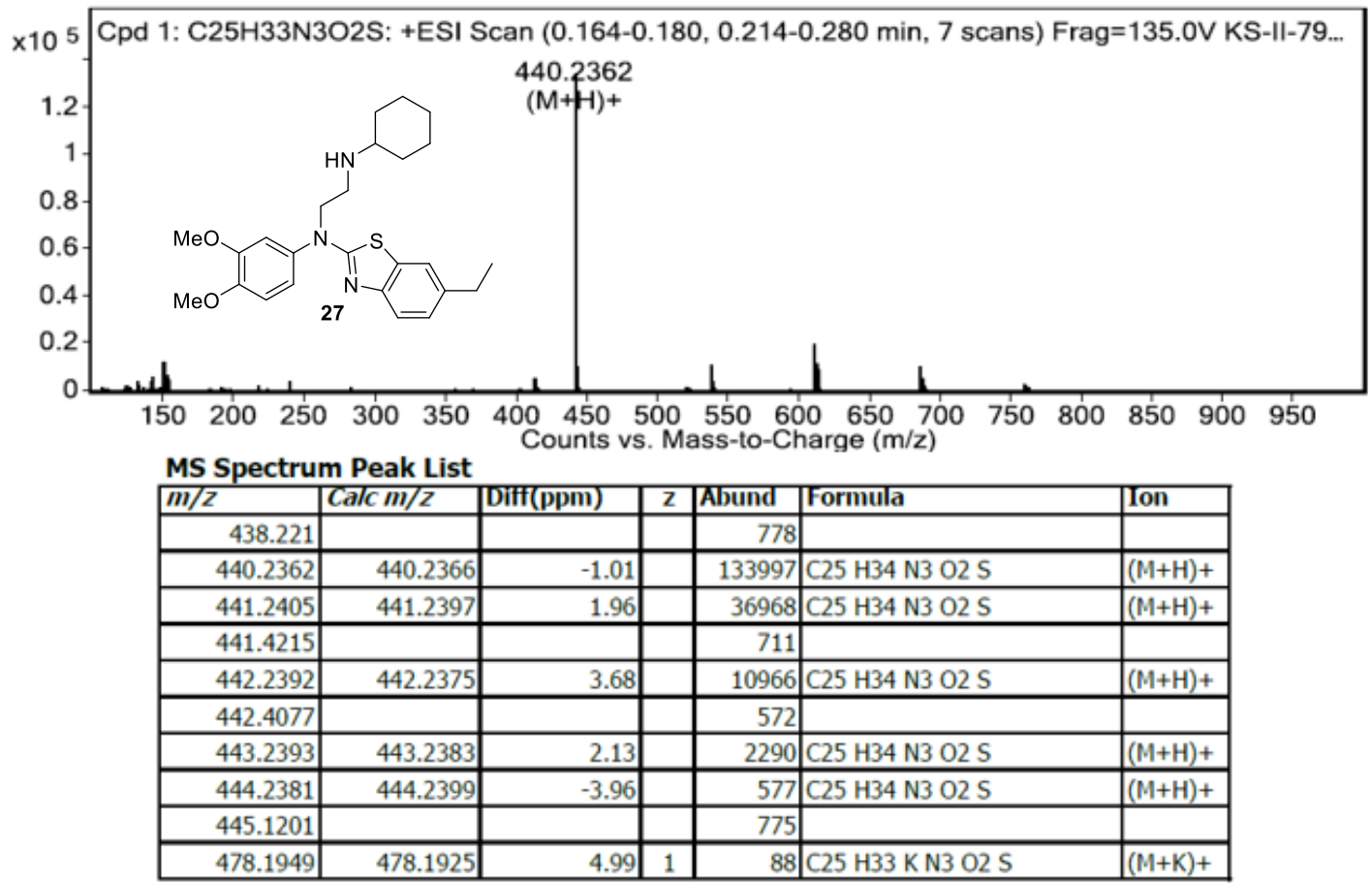


$N^{l}$-Cyclohexyl- $N^{3}$-(3,4-dimethoxyphenyl)- $N^{3}$-(6-ethylbenzo[d] thiazol-2-yl)propane-1,3-diamine (28)

HRMS (ESI): calcd. for $\mathrm{C}_{26} \mathrm{H}_{36} \mathrm{~N}_{3} \mathrm{O}_{2} \mathrm{~S}[\mathrm{M}+\mathrm{H}]^{+} 454.2523$, found 454.2517 .

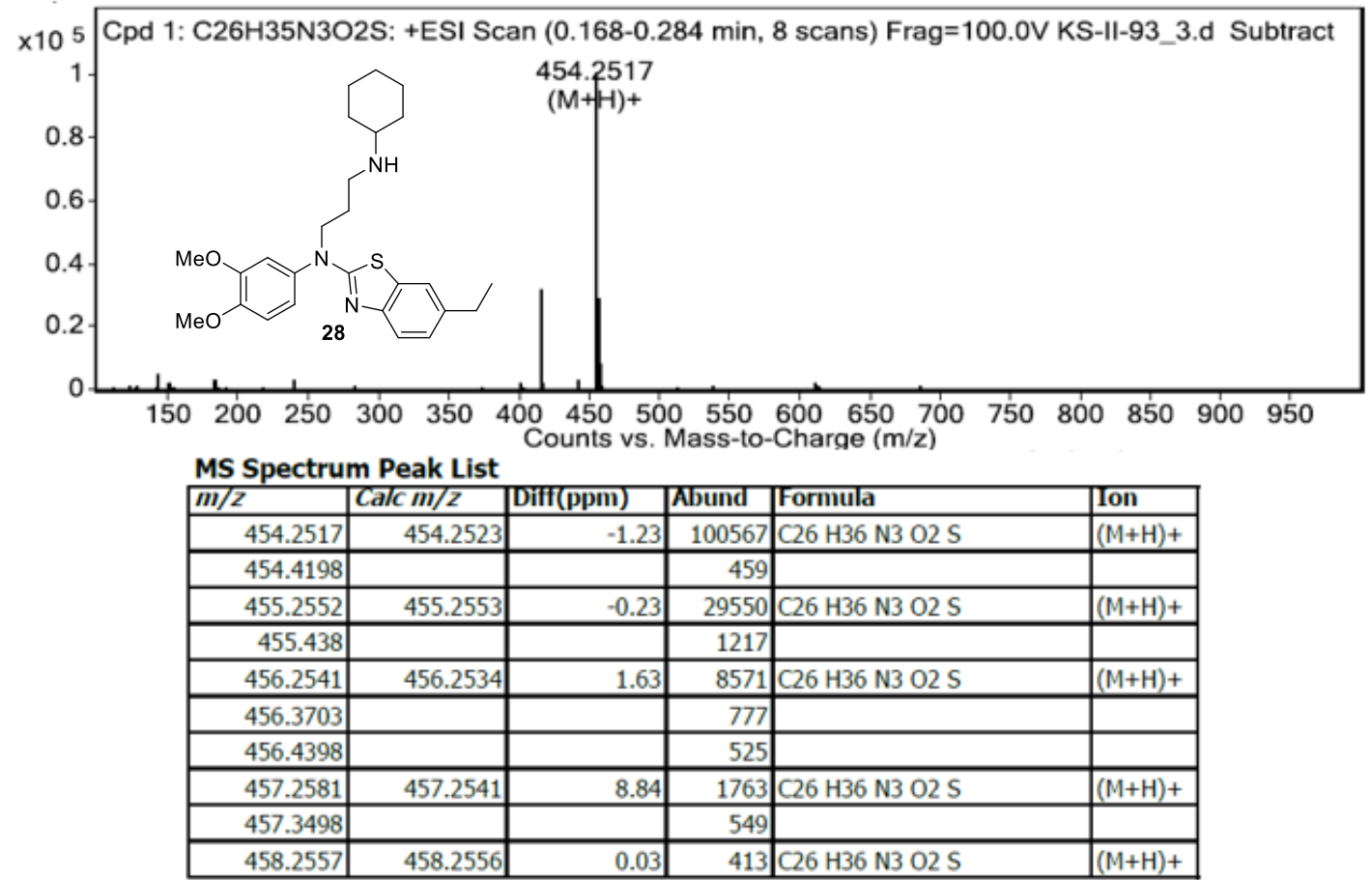

\section{$N^{I}$-Cyclohexyl- $N^{4}$-(3,4-dimethoxyphenyl)- $N^{4}$-(6-ethylbenzo $[d]$ thiazol-2-yl)butane-1,4-diamine} (29)

HRMS (ESI): calcd. for $\mathrm{C}_{27} \mathrm{H}_{38} \mathrm{~N}_{3} \mathrm{O}_{2} \mathrm{~S}[\mathrm{M}+\mathrm{H}]^{+}$468.2679, found 468.2675 .

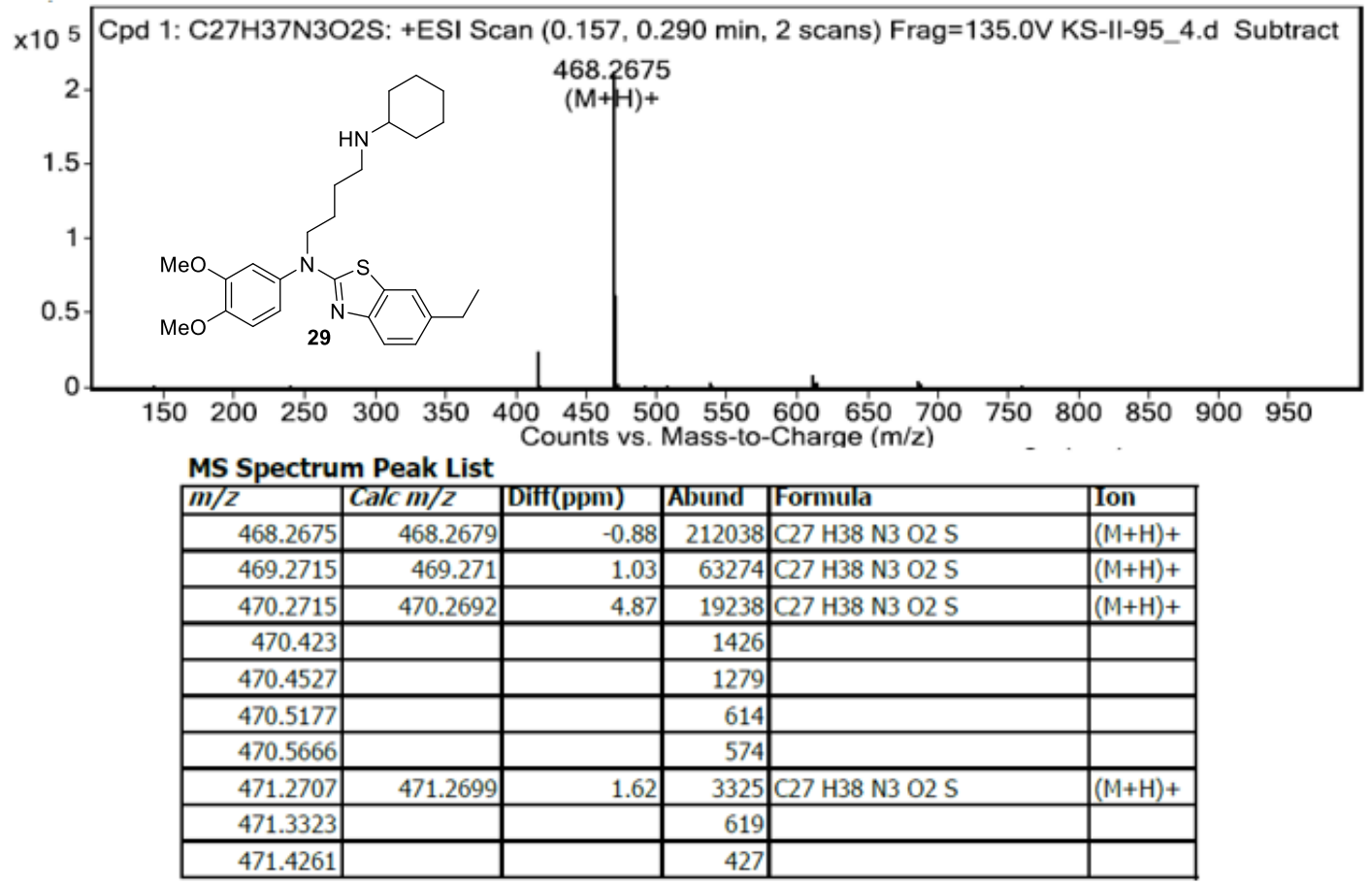



(30)

HRMS (ESI): calcd. for $\mathrm{C}_{28} \mathrm{H}_{40} \mathrm{~N}_{3} \mathrm{O}_{2} \mathrm{~S}[\mathrm{M}+\mathrm{H}]^{+}$482.2836, found 482.2833 .

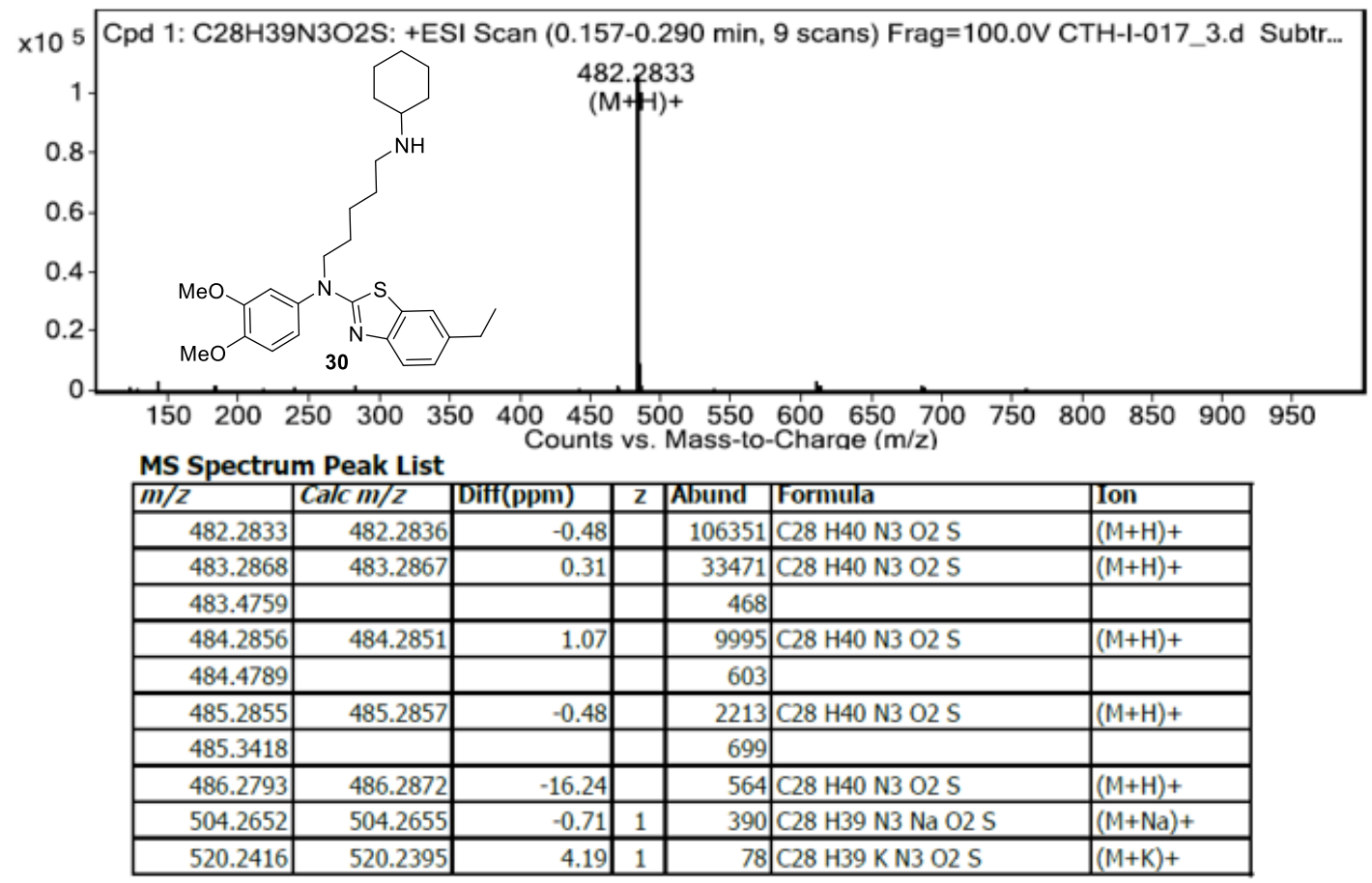



(31)

HRMS (FAB): calcd. for $\mathrm{C}_{24} \mathrm{H}_{32} \mathrm{~N}_{3} \mathrm{O}_{2} \mathrm{~S}[\mathrm{M}+\mathrm{H}]^{+} 426.2215$, found 426.2214 .

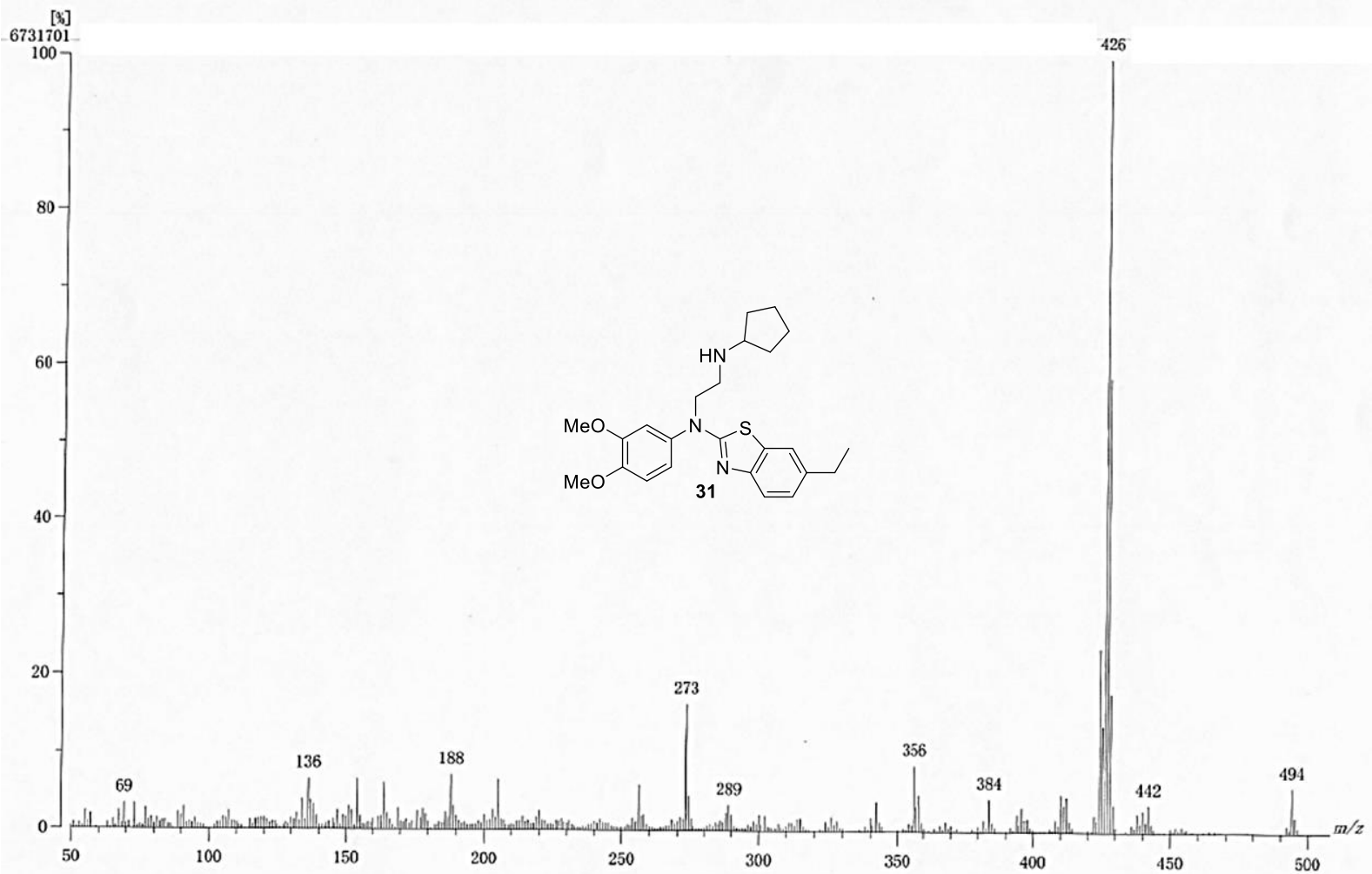

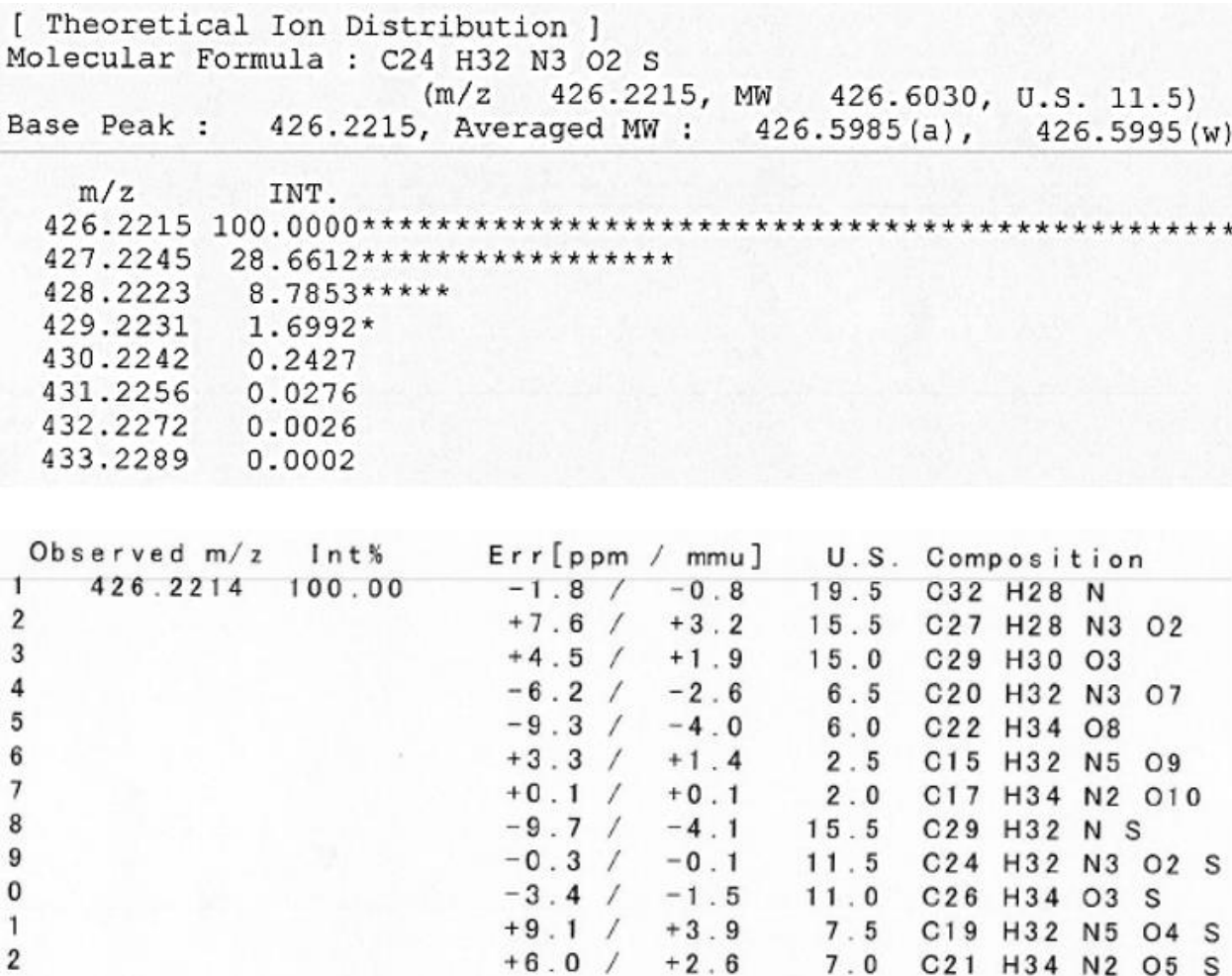


$N^{1}$-Cyclopentyl- $N^{3}$-(3,4-dimethoxyphenyl)- $N^{3}$-(6-ethylbenzo $[d]$ thiazol-2-yl)propane-1,3-diamine (32)

HRMS (ESI): calcd. for $\mathrm{C}_{25} \mathrm{H}_{34} \mathrm{~N}_{3} \mathrm{O}_{2} \mathrm{~S}[\mathrm{M}+\mathrm{H}]^{+} 440.2366$, found 440.2373 .

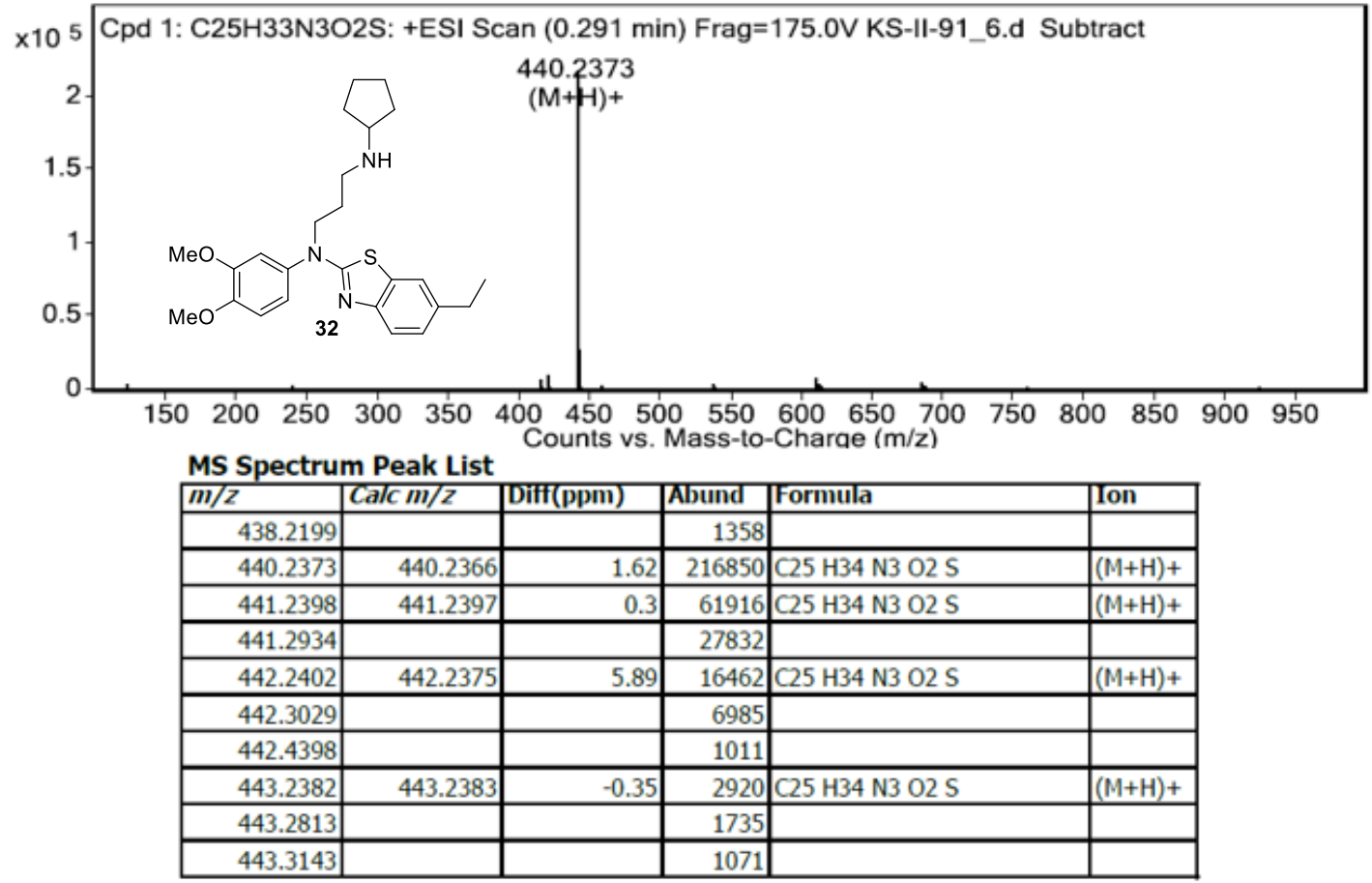




\section{$N^{I}$-Cyclopentyl- $N^{4}$-(3,4-dimethoxyphenyl)- $N^{4}$-(6-ethylbenzo $[d]$ thiazol-2-yl)butane-1,4-diamine} (33)

HRMS (FAB): calcd. for $\mathrm{C}_{26} \mathrm{H}_{36} \mathrm{~N}_{3} \mathrm{O}_{2} \mathrm{~S}[\mathrm{M}+\mathrm{H}]^{+}$454.2528, found 454.2527 .
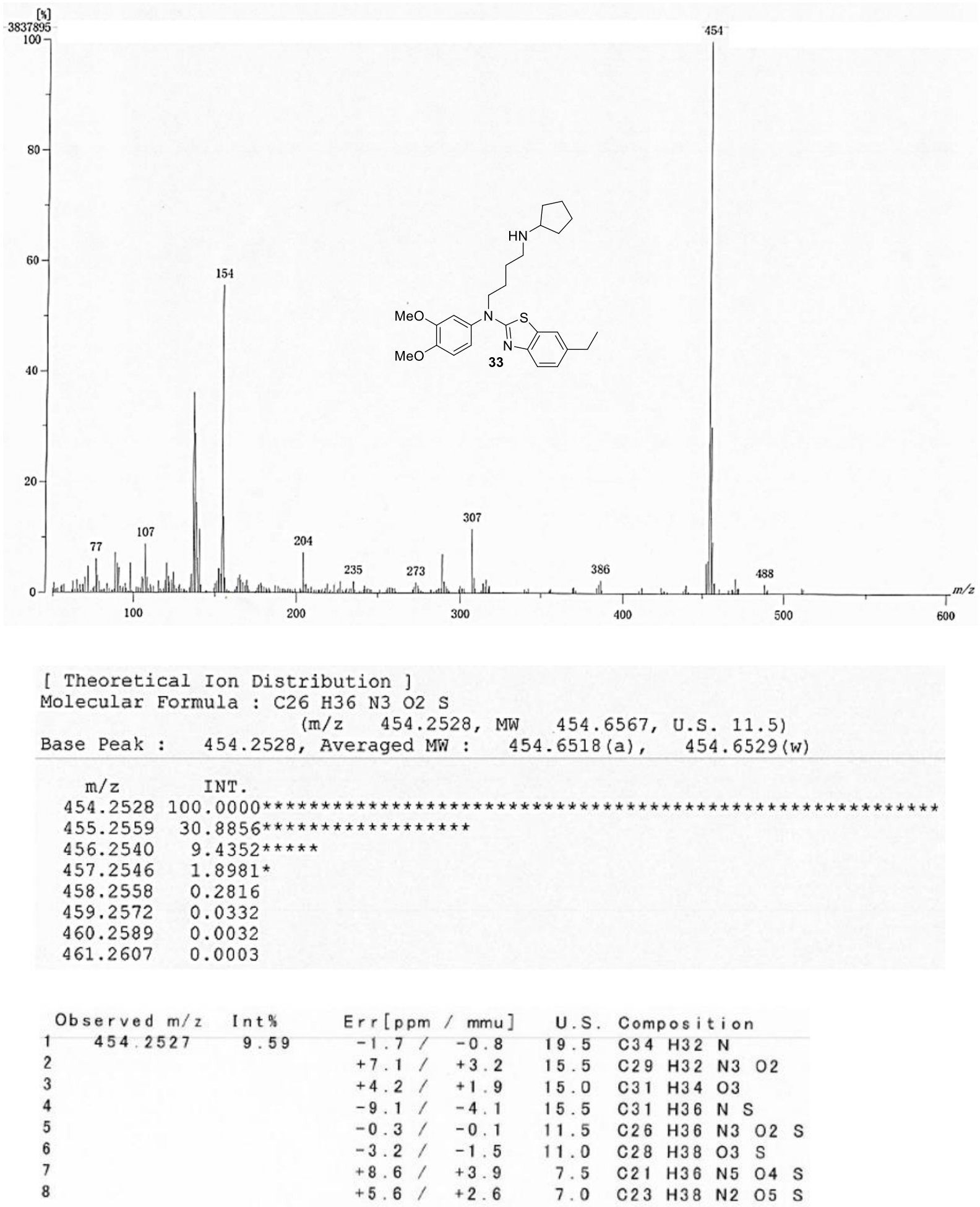
$N^{l}$-Cyclopentyl- $N^{5}$-(3,4-dimethoxyphenyl)- $N^{5}$-(6-ethylbenzo[d]thiazol-2-yl)pentane-1,5-diamine (34)

HRMS (ESI): calcd. for $\mathrm{C}_{27} \mathrm{H}_{38} \mathrm{~N}_{3} \mathrm{O}_{2} \mathrm{~S}[\mathrm{M}+\mathrm{H}]^{+}$468.2679, found 468.2697.

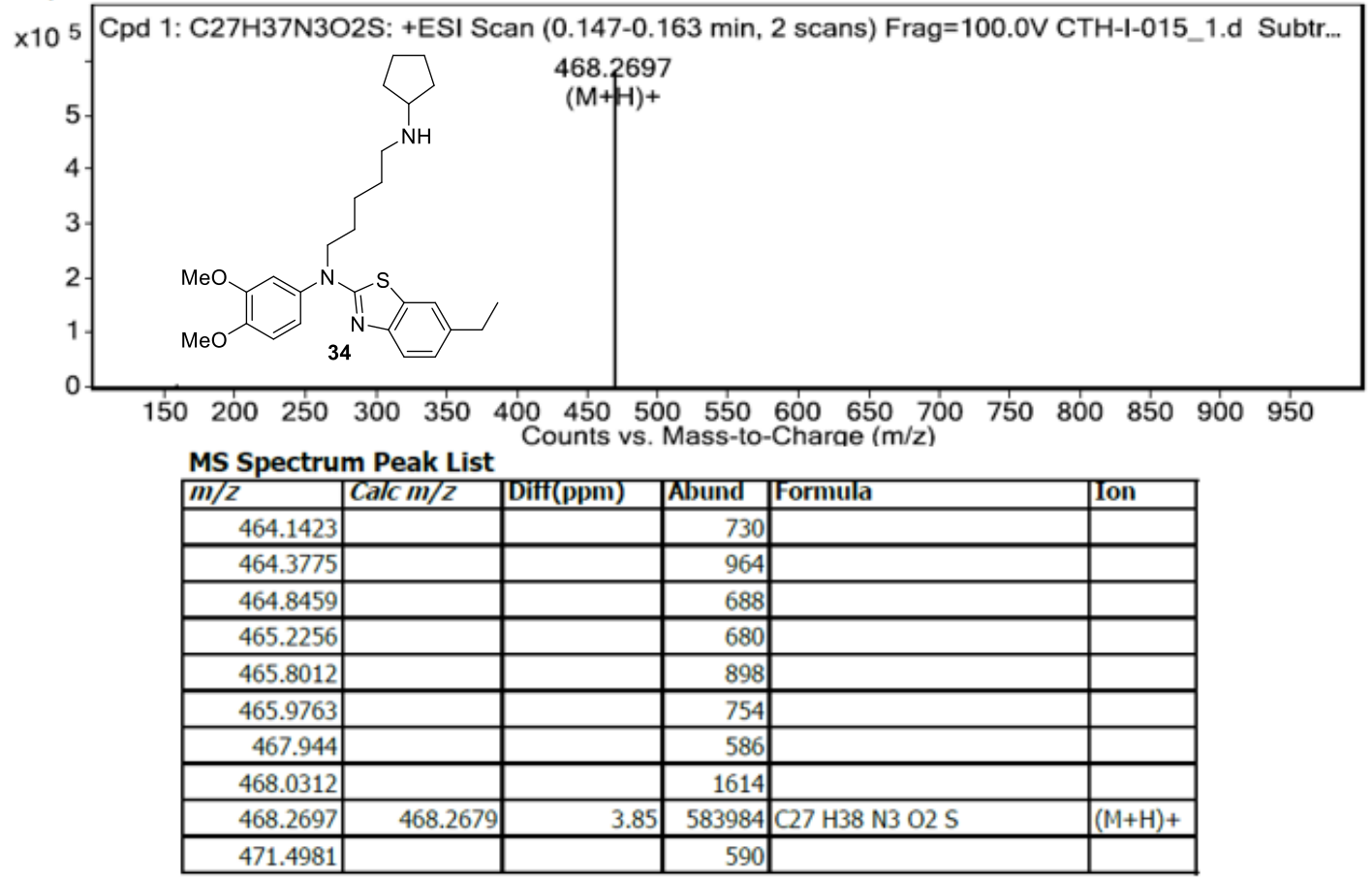




\section{HPLC Analysis of Final Target Compounds}

HPLC was performed using an Agilent 1200 Series HPLC system (Agilent Technologies, Palo Alto, CA, USA). Mobile phase A comprised $0.1 \%$ formic acid in HPLC-grade $\mathrm{H}_{2} \mathrm{O}$, and the reversed-phase Agilent Eclipse Plus C18 column $(4.6 \times 100 \mathrm{~mm}, 3.5 \mu \mathrm{m})$ had a flow rate of $0.7 \mathrm{~mL} / \mathrm{min}(30-100 \%$ aqueous $\mathrm{MeOH}$ with $0.1 \%$ formic acid over $20 \mathrm{~min}$ and $\mathrm{MeOH}$ with $0.1 \%$ formic acid from 20 to 25 $\min )$.

Table S2. Purity of all Biologically Evaluated Compounds

\begin{tabular}{|c|c|c|}
\hline Compound \# & Retention Time $t_{R}(\min )$ & Purity (\%) \\
\hline 4 & 16.080 & 98.4 \\
\hline 5 & 16.920 & 98.9 \\
\hline 6 & 16.666 & 96.7 \\
\hline 7 & 16.189 & 98.2 \\
\hline 8 & 15.447 & 99.4 \\
\hline 9 & 16.029 & 99.5 \\
\hline 10 & 16.461 & 99.8 \\
\hline 11 & 17.161 & 99.6 \\
\hline 12 & 16.954 & 98.8 \\
\hline 13 & 17.100 & 97.6 \\
\hline 14 & 17.842 & 99.1 \\
\hline 15 & 14.946 & 97.0 \\
\hline 16 & 16.333 & 99.7 \\
\hline 17 & 16.528 & 99.1 \\
\hline 18 & 16.987 & 99.5 \\
\hline 19 & 15.081 & 96.1 \\
\hline 20 & 16.338 & 99.5 \\
\hline 21 & 16.237 & 99.5 \\
\hline 22 & 16.781 & 99.6 \\
\hline 23 & 14.866 & 97.6 \\
\hline 24 & 16.460 & 99.6 \\
\hline 25 & 16.388 & 99.8 \\
\hline 26 & 17.094 & 96.2 \\
\hline 27 & 17.405 & 99.7 \\
\hline 28 & 17.490 & 98.5 \\
\hline 29 & 17.372 & 95.4 \\
\hline 30 & 17.870 & 98.6 \\
\hline 31 & 17.175 & 98.9 \\
\hline 32 & 17.112 & 97.9 \\
\hline 33 & 19.032 & 98.7 \\
\hline 34 & 17.364 & 98.6 \\
\hline
\end{tabular}


$\mathrm{N}$-(3,4-Dimethoxyphenyl)-6-ethyl- $\mathrm{N}$-((1-(2-(piperidin-1-yl)ethyl)-1H-1,2,3-triazol-4yl)methyl)benzo $[d]$ thiazol-2-amine (4)

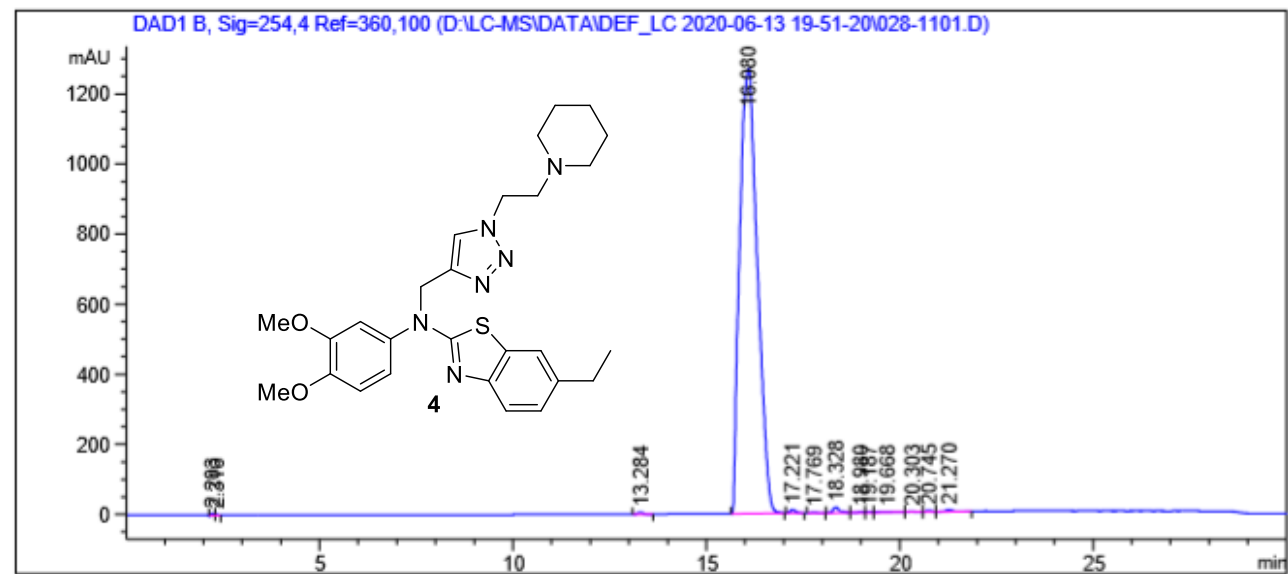

\begin{tabular}{|c|c|c|c|c|c|c|}
\hline $\begin{array}{c}\text { Peak } \\
\#\end{array}$ & $\begin{array}{c}\text { RetTime } \\
\text { [min] }\end{array}$ & Type & $\begin{array}{l}\text { Width } \\
\text { [min] }\end{array}$ & $\begin{array}{c}\text { Area } \\
{[\mathrm{mAU} * \mathrm{~s}]}\end{array}$ & $\begin{array}{l}\text { Height } \\
\text { [mAd] }\end{array}$ & $\begin{array}{c}\text { Area } \\
\text { sक }\end{array}$ \\
\hline & & & & ---------- & $\mid--------$ & ------ \\
\hline 1 & 2.203 & BV & 0.0683 & 25.66534 & 5.72521 & 0.0629 \\
\hline 2 & 2.316 & vV & 0.0759 & 17.23353 & 3.59471 & 0.0422 \\
\hline 3 & 13.284 & $\mathrm{BB}$ & 0.1750 & 48.33041 & 4.43914 & 0.1184 \\
\hline 4 & 16.080 & $\mathrm{BB}$ & 0.4529 & $4.01711 \mathrm{e} 4$ & 1270.37952 & 98.4271 \\
\hline 5 & 17.221 & $\mathrm{BB}$ & 0.1641 & 105.95334 & 9.95748 & 0.2596 \\
\hline 6 & 17.769 & $\mathrm{BB}$ & 0.1306 & 17.63392 & 1.93077 & 0.0432 \\
\hline 7 & 18.328 & $\mathrm{BB}$ & 0.1877 & 195.67355 & 15.67187 & 0.4794 \\
\hline 8 & 18.980 & BV & 0.2118 & 27.08732 & 1.83951 & 0.0664 \\
\hline 9 & 19.187 & vV & 0.1681 & 17.62062 & 1.55627 & 0.0432 \\
\hline 10 & 19.668 & vV & 0.3539 & 51.13960 & 1.83061 & 0.1253 \\
\hline 11 & 20.303 & vV & 0.1682 & 25.72149 & 2.08141 & 0.0630 \\
\hline 12 & 20.745 & vV & 0.1555 & 39.52024 & 4.06267 & 0.0968 \\
\hline 13 & 21.270 & VB & 0.2214 & 70.37014 & 4.67960 & 0.1724 \\
\hline
\end{tabular}


$N$-((1-(2-(Cyclopentylamino)ethyl)-1H-1,2,3-triazol-4-yl)methyl)- $N$-(3,4-dimethoxyphenyl)-6ethylbenzo $[d]$ thiazol-2-amine (5)

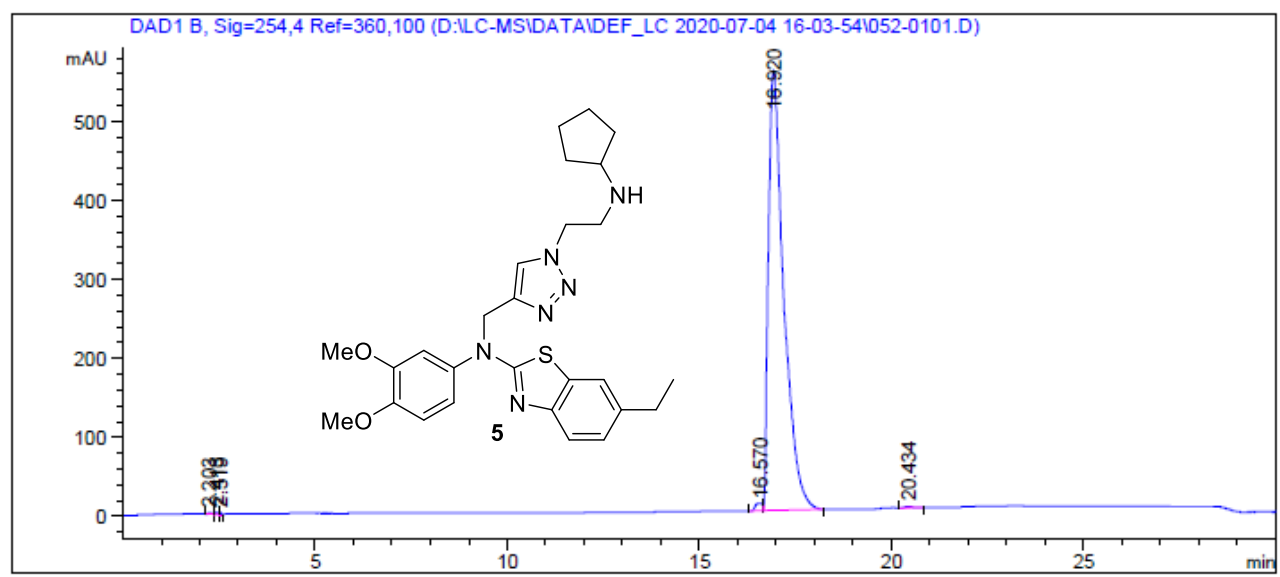

\begin{tabular}{|c|c|c|c|c|c|c|}
\multicolumn{1}{l}{ \# } & \multicolumn{1}{c}{ Time } & \multicolumn{1}{c}{ Area } & \multicolumn{1}{c}{ Height } & \multicolumn{1}{c}{ Width } & \multicolumn{1}{c|}{ Area\% } & Symmetry \\
\hline 1 & 2.203 & 11.7 & 1.4 & 0.1071 & 0.075 & 0.147 \\
\hline 2 & 2.413 & 15.6 & 3.1 & 0.0735 & 0.100 & 0.887 \\
\hline 3 & 2.519 & 8.8 & 2.5 & 0.0543 & 0.056 & 1.192 \\
\hline 4 & 16.57 & 122.1 & 9.2 & 0.1782 & 0.783 & 1.803 \\
\hline 5 & 16.92 & 15414.3 & 557.2 & 0.3987 & 98.858 & 0.422 \\
\hline 6 & 20.434 & 19.9 & 1.2 & 0.2447 & 0.128 & 0.639 \\
\hline
\end{tabular}

$N$-(3,4-Dimethoxyphenyl)-6-ethyl- $N$-((1-(2-(propylamino)ethyl)-1H-1,2,3-triazol-4yl)methyl)benzo[d]thiazol-2-amine (6)

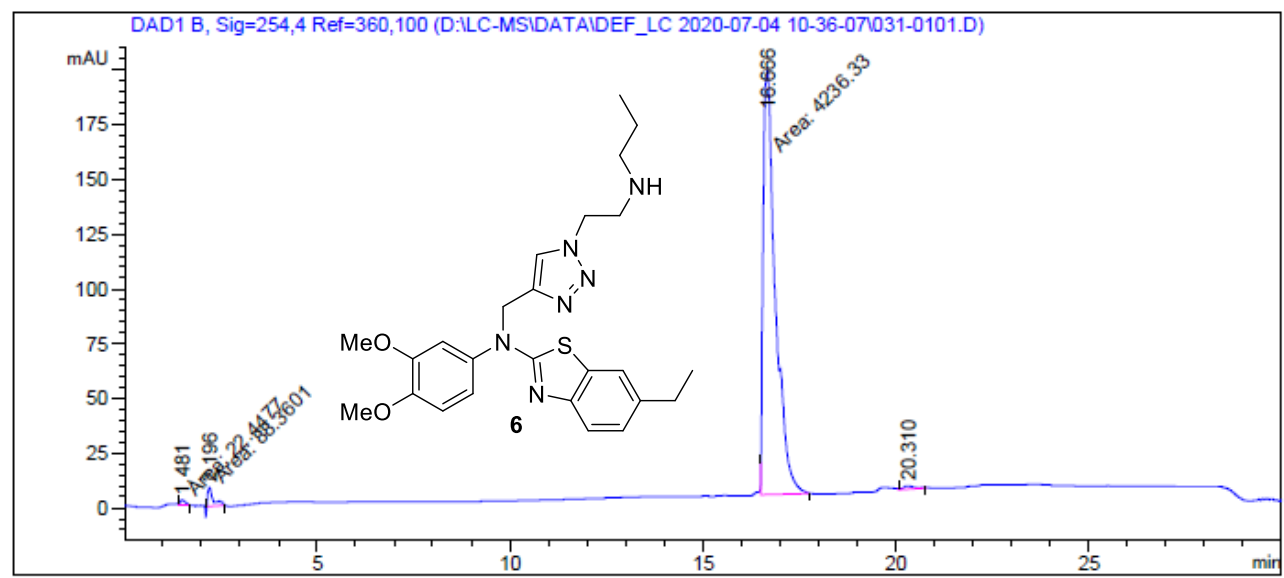

\begin{tabular}{|c|c|c|c|c|c|c|}
\hline$\#$ & Time & Area & Height & Width & Area\% & Symmetry \\
\hline 1 & 1.481 & 22.4 & 2.5 & 0.1501 & 0.513 & 0.44 \\
\hline 2 & 2.196 & 88.4 & 8.6 & 0.1703 & 2.018 & 0.417 \\
\hline 3 & 16.666 & 4236.3 & 193.7 & 0.3645 & 96.737 & 0.491 \\
\hline 4 & 20.31 & 32.1 & 1.6 & 0.2794 & 0.732 & 0.787 \\
\hline
\end{tabular}


$N$-(3,4-Dimethoxyphenyl)-6-ethyl- $N$-((1-(2-(pyrrolidin-1-yl)ethyl)-1H-1,2,3-triazol-4yl)methyl)benzo[d]thiazol-2-amine (7)

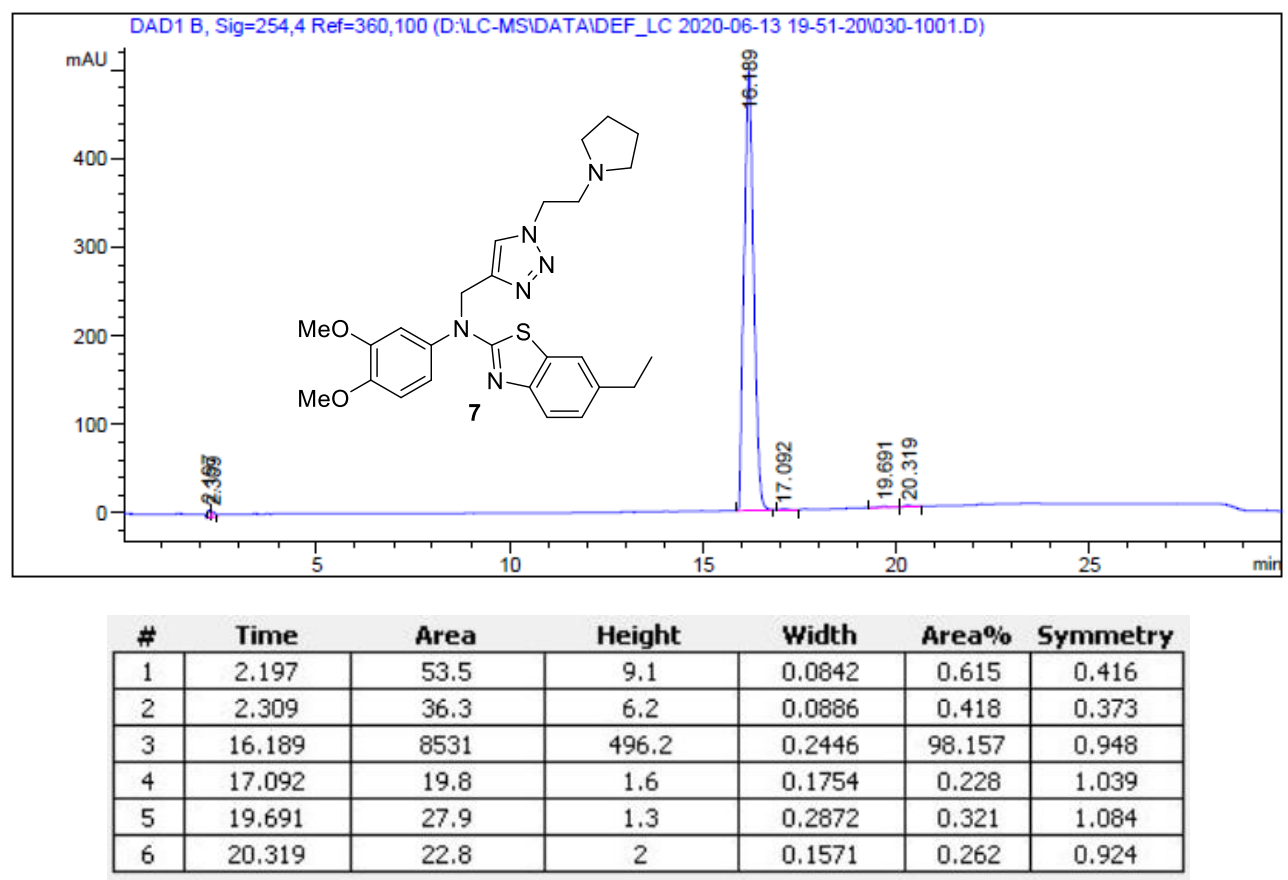

$N$-(3,4-Dimethoxyphenyl)-6-ethyl- $N$-(2-(piperidin-1-yl)ethyl)benzo[ $d]$ thiazol-2-amine (8)

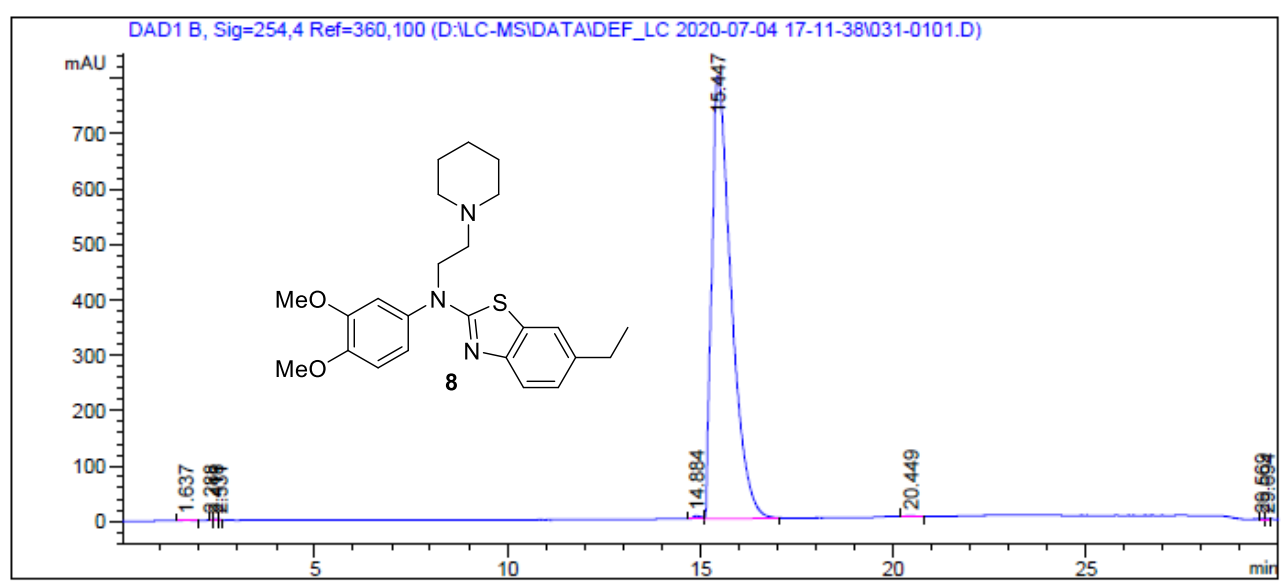

\begin{tabular}{|c|c|c|c|c|c|c|}
\hline$\#$ & Time & Area & Height & Width & Area $\%$ & 5ymmetry \\
\hline 1 & 1.637 & 17.2 & 1.4 & 0.1905 & 0.061 & \begin{tabular}{|l|}
0.56 \\
\end{tabular} \\
\hline 2 & 2.288 & 9.2 & 1.6 & 0.081 & 0.033 & 0.838 \\
\hline 3 & 2.418 & 17.9 & 3.2 & 0.0785 & 0.064 & 0.831 \\
\hline 4 & 2.531 & 7.8 & 2.3 & 0.0509 & 0.028 & 1.194 \\
\hline 5 & 14.884 & 73 & 5.3 & 0.1959 & 0.260 & 0.491 \\
\hline 6 & 15.447 & 27913 & 800.3 & 0.4789 & 99.428 & 0.421 \\
\hline 7 & 20.449 & 21 & 1.2 & 0.2294 & 0.075 & 0.776 \\
\hline 8 & 29.569 & 6 & 1.1 & 0.0802 & 0.021 & 1.009 \\
\hline 9 & 29.694 & 8.5 & 1.1 & 0.1071 & 0.030 & 0.812 \\
\hline
\end{tabular}


$N$-(3,4-Dimethoxyphenyl)-6-ethyl- $N$-(3-(piperidin-1-yl)propyl)benzo[d]thiazol-2-amine (9)

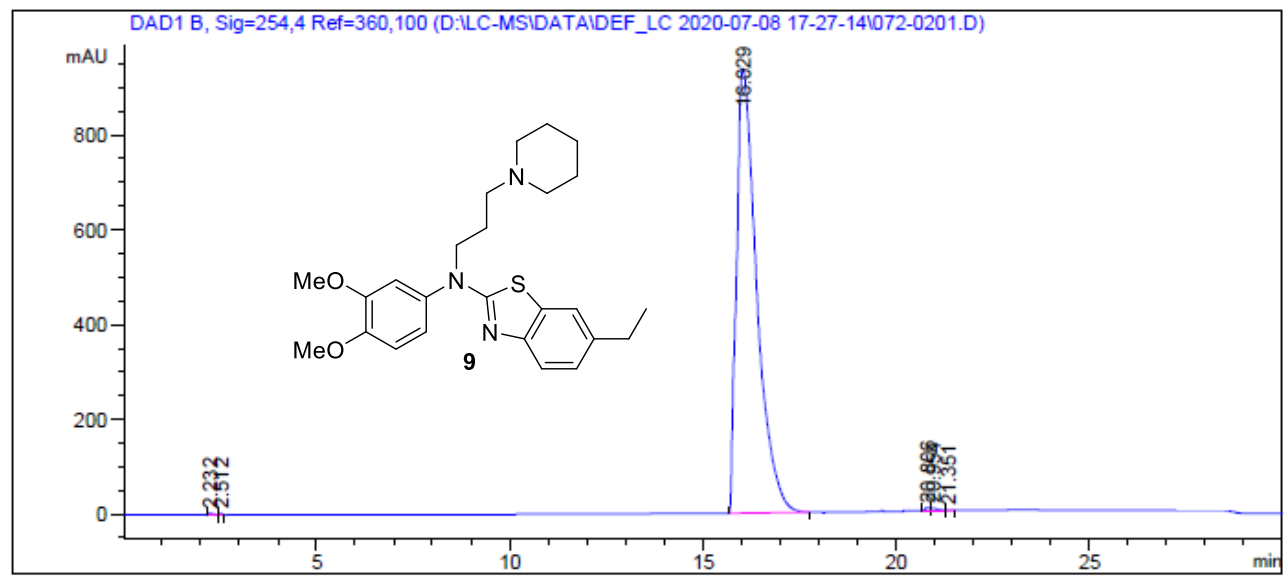

\begin{tabular}{|c|c|c|c|c|c|c|}
\hline$\#$ & Time & Area & Height & Width & Area $\%$ & 5ymmetry \\
\hline 1 & 2.232 & 24.7 & 3.2 & 0.114 & 0.072 & 0.178 \\
\hline 2 & 2.512 & 15.5 & 3.1 & 0.0801 & 0.045 & 1.17 \\
\hline 3 & 16.029 & 34136.1 & 938.5 & 0.5204 & 99.460 & 0.418 \\
\hline 4 & 20.806 & 62.1 & 6.8 & 0.1367 & 0.181 & 0.873 \\
\hline 5 & 20.954 & 72.7 & 5.6 & 0.1768 & 0.212 & 0.3 \\
\hline 6 & 21.351 & 10.4 & 1 & 0.1321 & 0.030 & 0.51 \\
\hline
\end{tabular}

$N$-(3,4-Dimethoxyphenyl)-6-ethyl- $N$-(4-(piperidin-1-yl)butyl)benzo[d] thiazol-2-amine (10)

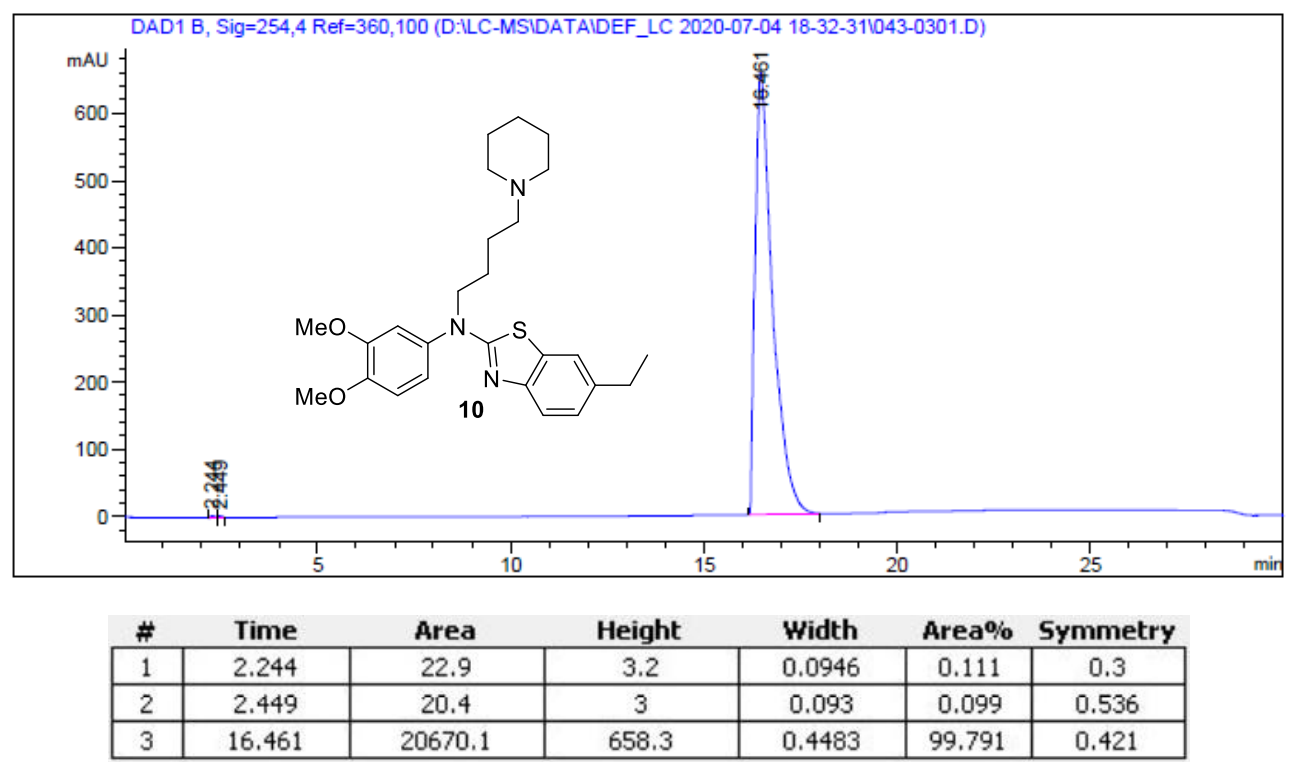




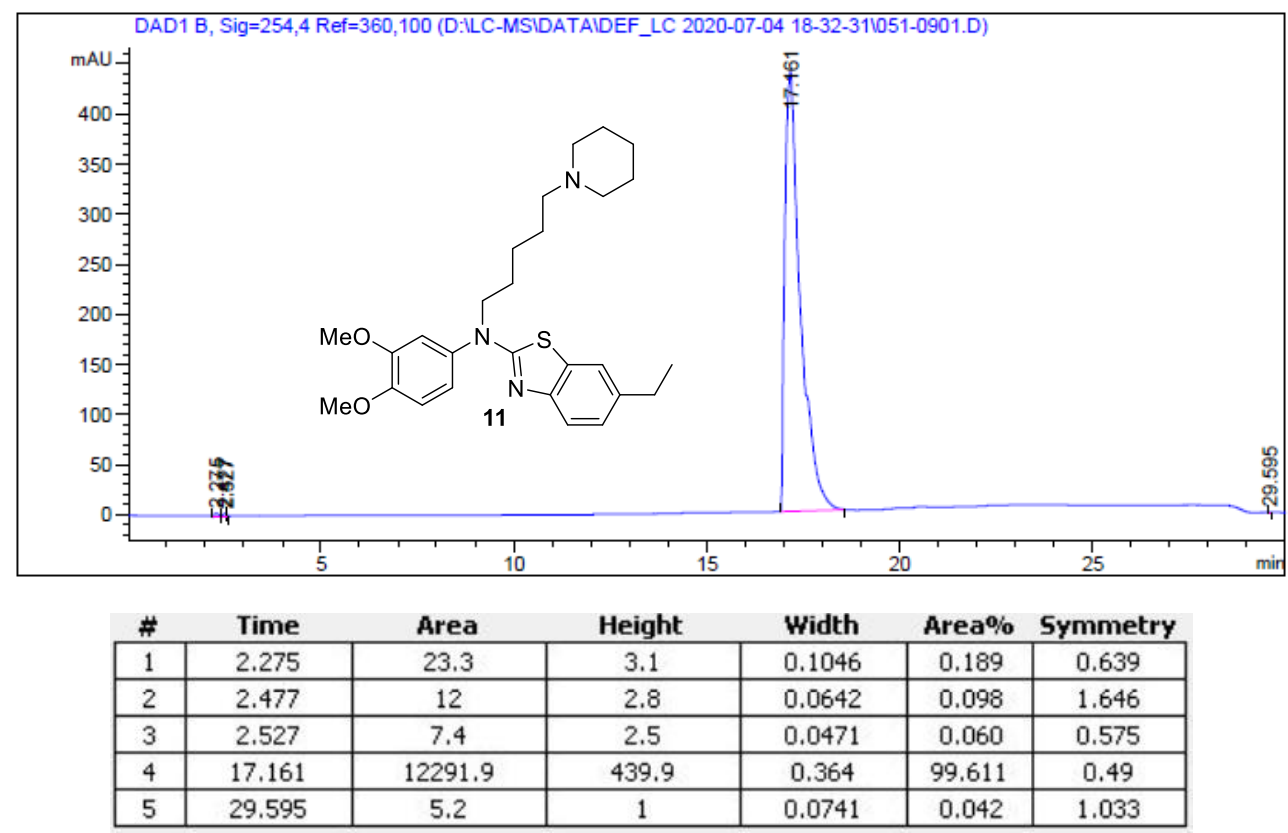

6-Ethyl- $N$-(3-methoxyphenyl)- $N$-(3-(piperidin-1-yl)propyl)benzo[d] thiazol-2-amine (12)

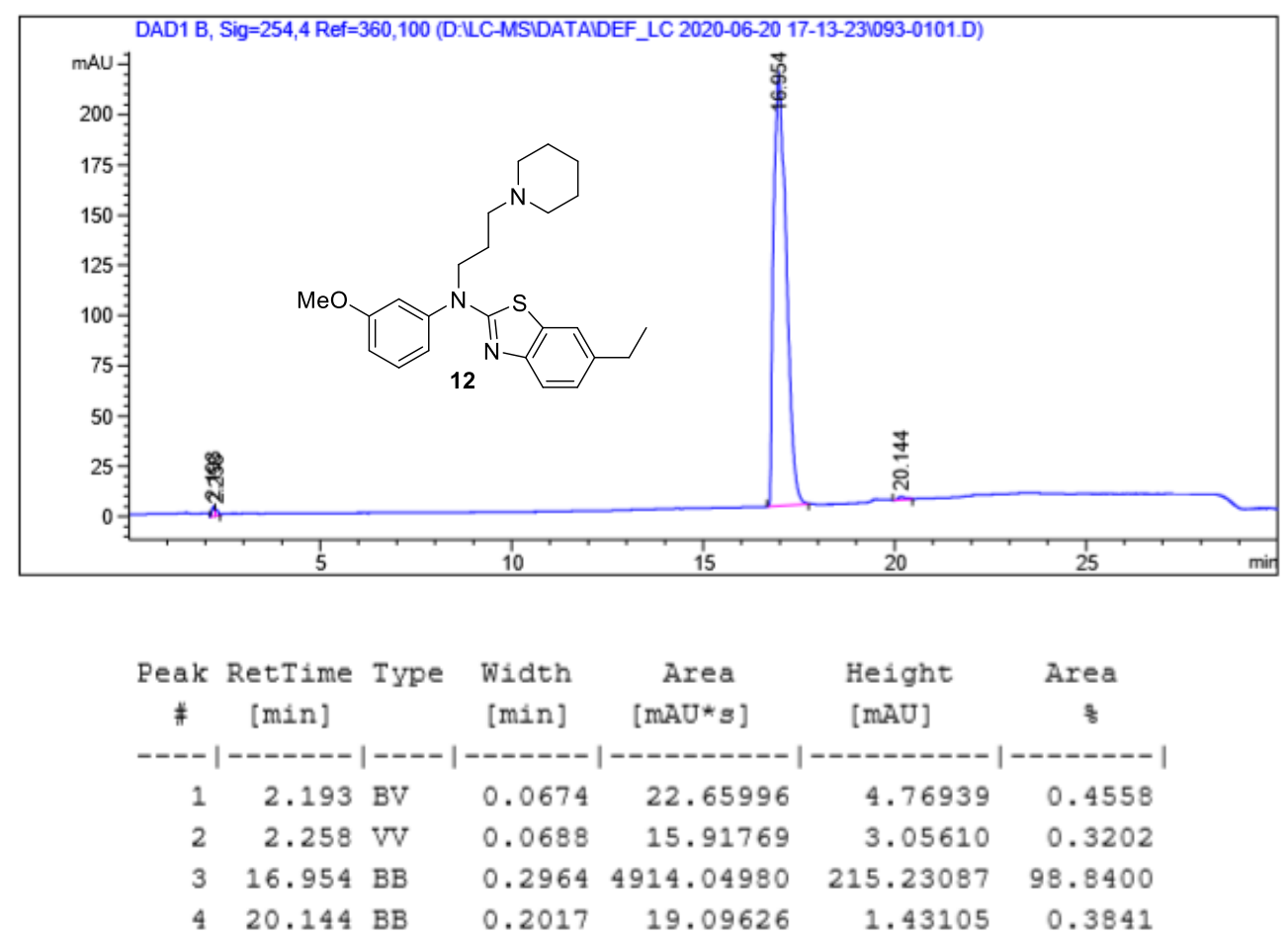


$N$-(3,4-Difluorophenyl)-6-ethyl- $N$-(3-(piperidin-1-yl)propyl)benzo[d] thiazol-2-amine (13)

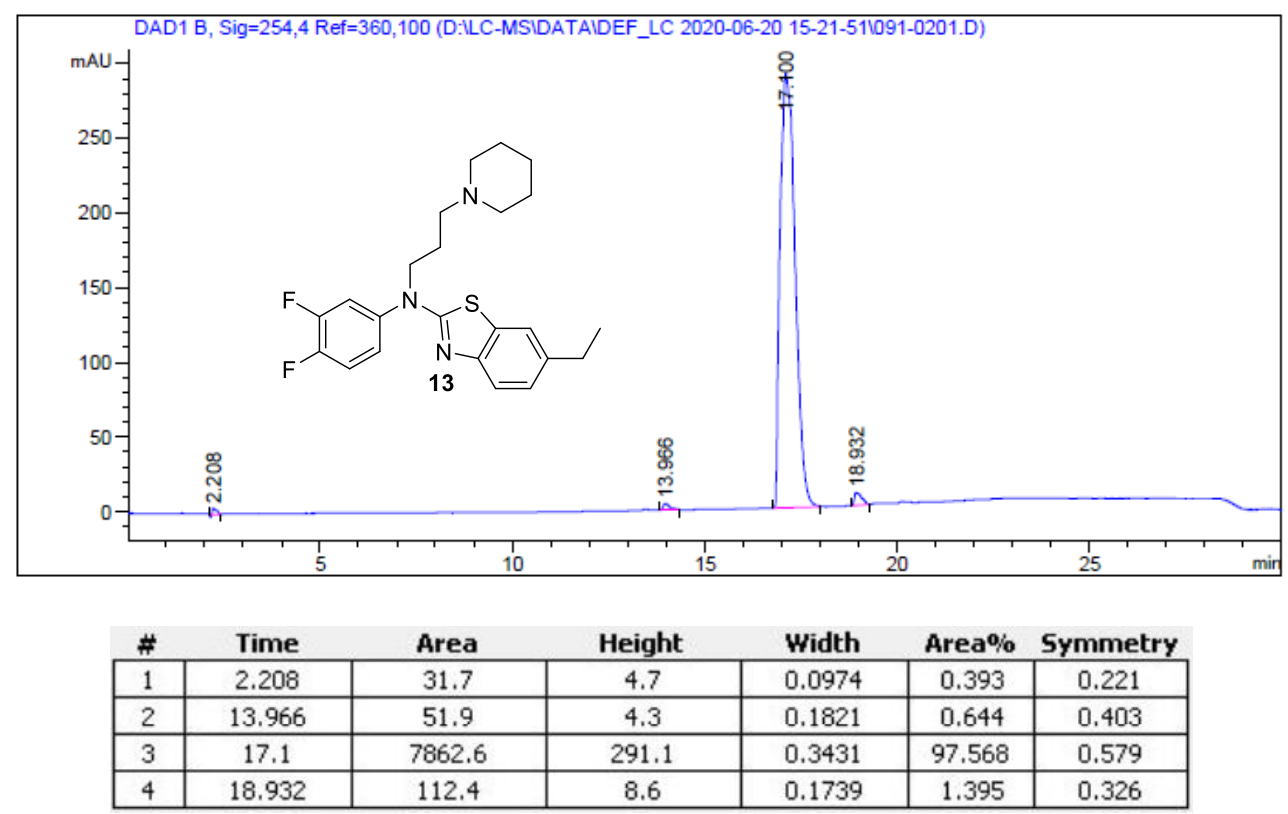

6-Ethyl- $N$-(3-(piperidin-1-yl)propyl)- $N$-(4-(trifluoromethyl)phenyl)benzo[d] thiazol-2-amine (14)

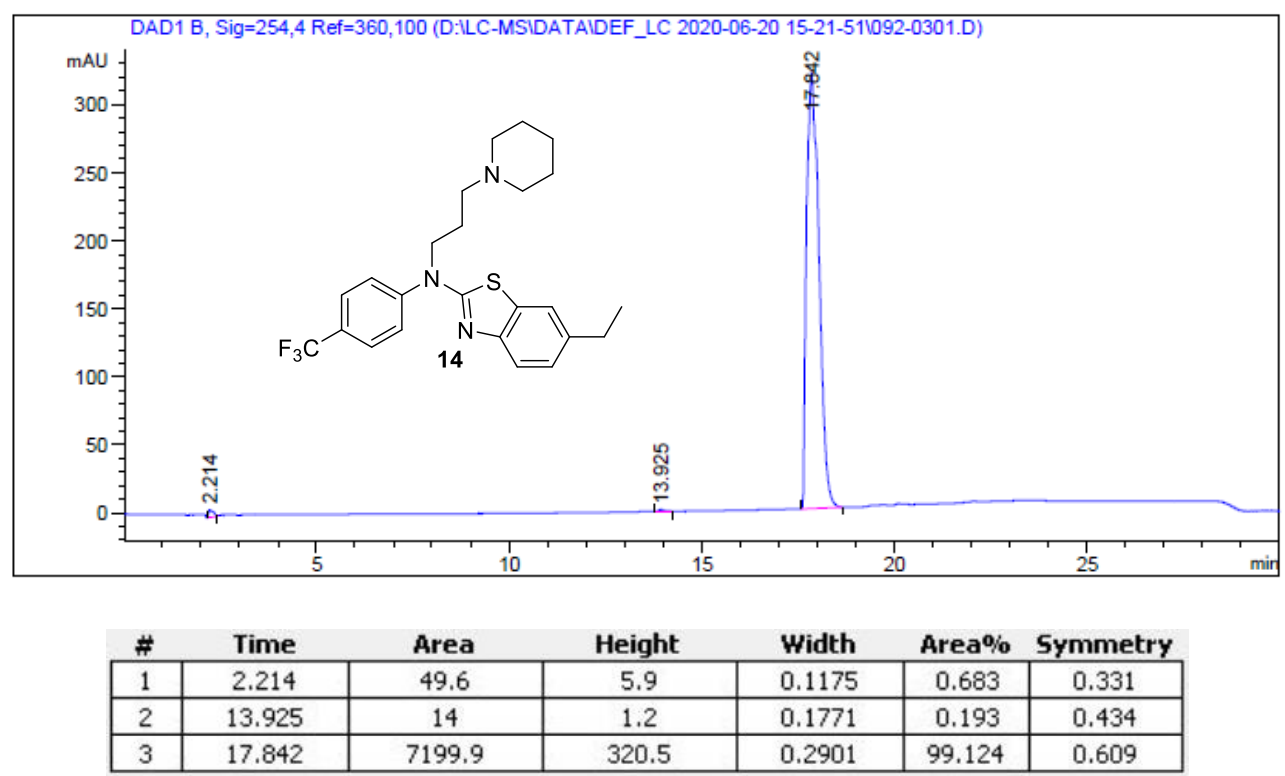


$N$-(3,4-Dimethoxyphenyl)-6-ethyl- $N$-(2-(pyrrolidin-1-yl)ethyl)benzo[d] thiazol-2-amine (15)

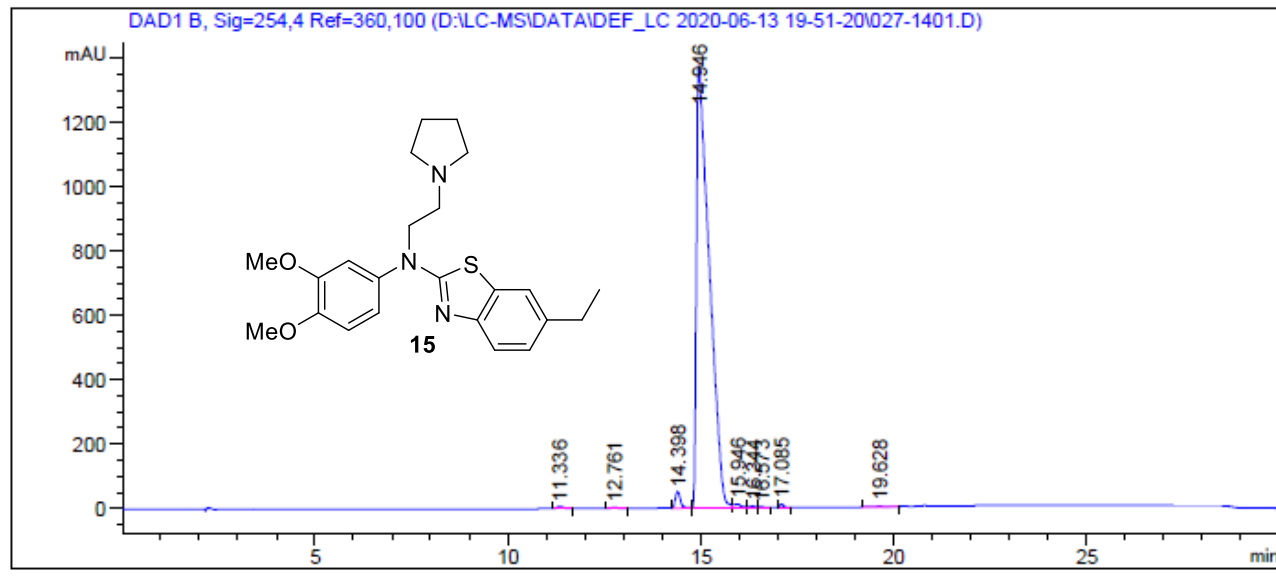

\begin{tabular}{|c|c|c|c|c|c|c|}
\hline$\#$ & Time & Area & Height & Width & Area $\%$ & Symmetry \\
\hline 1 & 11.336 & 81.6 & 7.3 & 0.1784 & 0.250 & \begin{tabular}{|l}
0.635 \\
\end{tabular} \\
\hline 2 & 12.761 & 41.1 & 3.9 & 0.171 & 0.126 & 1.01 \\
\hline 3 & 14.398 & 475.8 & 50.3 & 0.1465 & 1.458 & 0.783 \\
\hline 4 & 14.946 & 31659.9 & 1370 & 0.3296 & 97.038 & 0.221 \\
\hline 5 & 15.946 & 165.6 & 11.7 & 0.189 & 0.508 & 0.988 \\
\hline 6 & 16.344 & 59.8 & 5.2 & 0.1626 & 0.183 & 1.468 \\
\hline 7 & 16.573 & 30.5 & 2.6 & 0.1653 & 0.094 & 0.608 \\
\hline 8 & 17.085 & 73.8 & 11 & 0.1025 & 0.226 & 0.665 \\
\hline 9 & 19.628 & 38.3 & 1.9 & 0.2773 & 0.117 & 0.821 \\
\hline
\end{tabular}

$N$-(3,4-Dimethoxyphenyl)-6-ethyl- $N$-(3-(pyrrolidin-1-yl)propyl)benzo[d] thiazol-2-amine (16)

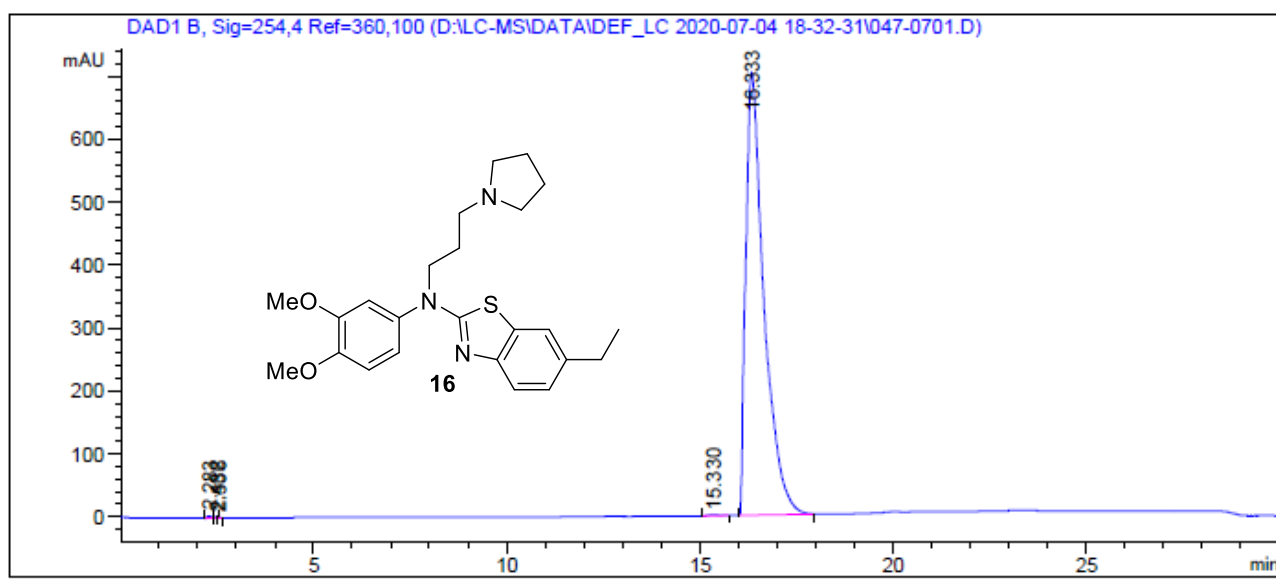

\begin{tabular}{|c|c|c|c|c|c|c|}
\hline \# & Time & Area & Height & Width & Area $\%$ & Symmetry \\
\hline 1 & 2.283 & 24.5 & 3.3 & 0.1015 & 0.107 & 0.57 \\
\hline 2 & 2.482 & 11.9 & 2.8 & 0.0612 & 0.052 & 1.501 \\
\hline 3 & 2.536 & 7.8 & 2.5 & 0.0481 & 0.034 & 0.684 \\
\hline 4 & 15.33 & 17.7 & 1 & 0.2236 & 0.077 & 0.762 \\
\hline 5 & 16.333 & 22922 & 703.9 & 0.4599 & 99.731 & 0.427 \\
\hline
\end{tabular}


$N$-(3,4-Dimethoxyphenyl)-6-ethyl- $N$-(4-(pyrrolidin-1-yl)butyl)benzo[d] thiazol-2-amine (17)

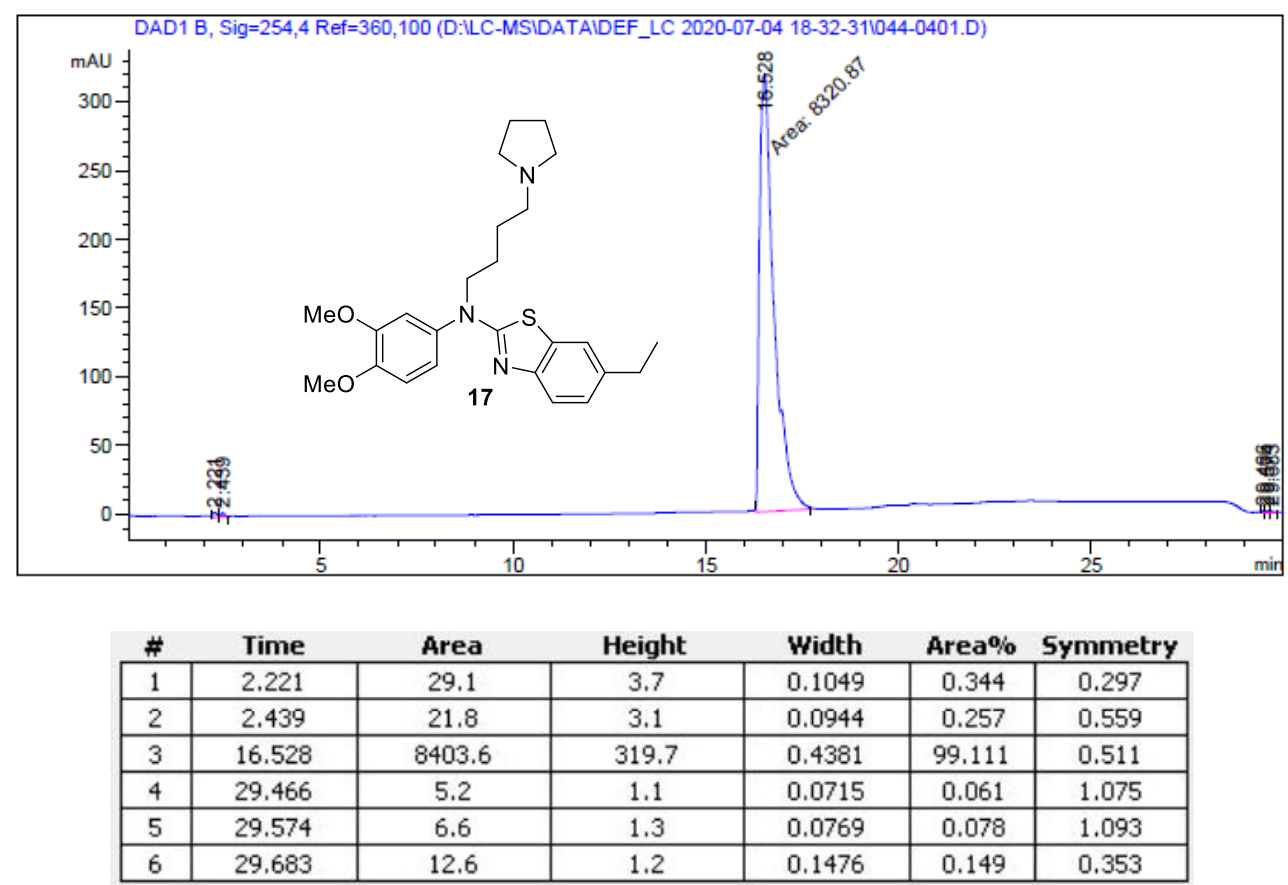

$N$-(3,4-Dimethoxyphenyl)-6-ethyl- $N$-(5-(pyrrolidin-1-yl)pentyl)benzo[d] thiazol-2-amine (18)

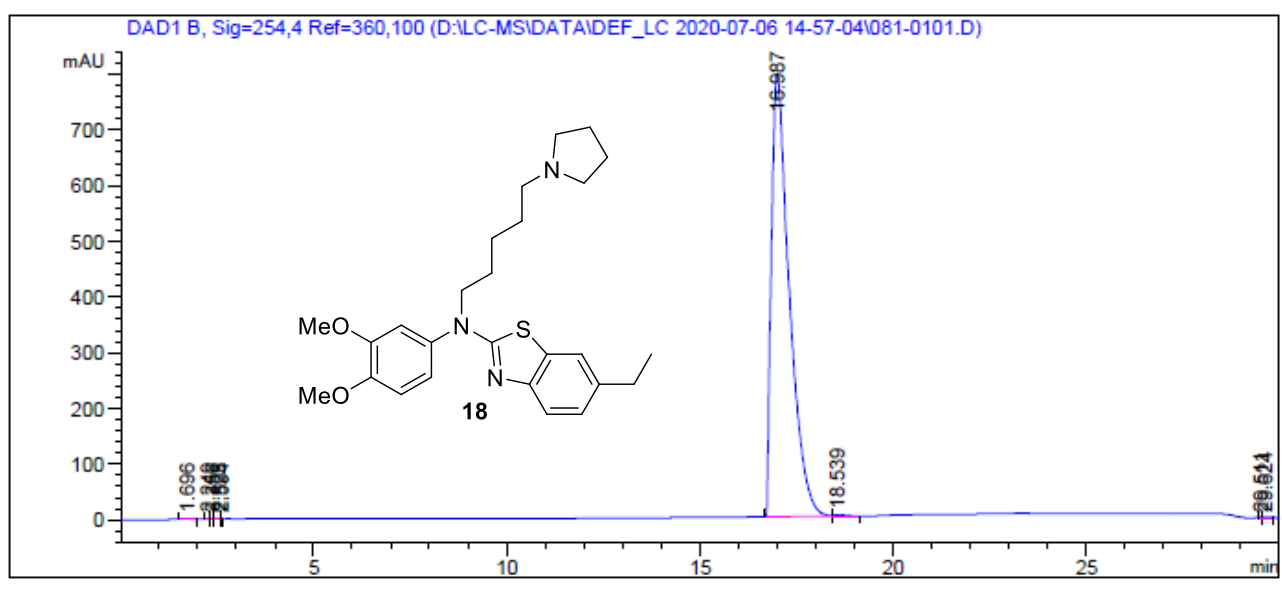

\begin{tabular}{|c|c|c|c|c|c|c|}
\hline$\#$ & Time & Area & Height & Width & Area $\%$ & Symmetry \\
\hline 1 & 1.696 & 14.9 & 1.4 & 0.1651 & 0.058 & 0.68 \\
\hline 2 & 2.249 & 5.8 & 1.3 & 0.0637 & 0.023 & 0.289 \\
\hline 3 & 2.356 & 6.6 & 1.3 & 0.0718 & 0.026 & 0.891 \\
\hline 4 & 2.525 & 14.3 & 2.3 & 0.0855 & 0.056 & 2.258 \\
\hline 5 & 2.594 & 6.2 & 1.9 & 0.0491 & 0.024 & 0.975 \\
\hline 6 & 16.987 & 25512.8 & 795.6 & 0.4701 & 99.545 & 0.4 \\
\hline 7 & 18.539 & 50.1 & 2.3 & 0.2957 & 0.196 & 0.338 \\
\hline 8 & 29.511 & 5.3 & 1.1 & 0.0706 & 0.021 & 1.048 \\
\hline 9 & 29.624 & 13.3 & 1.1 & 0.16 & 0.052 & 0.294 \\
\hline
\end{tabular}


$N$-(3,4-Dimethoxyphenyl)-6-ethyl- $N$-(2-morpholinoethyl)benzo[d] thiazol-2-amine (19)

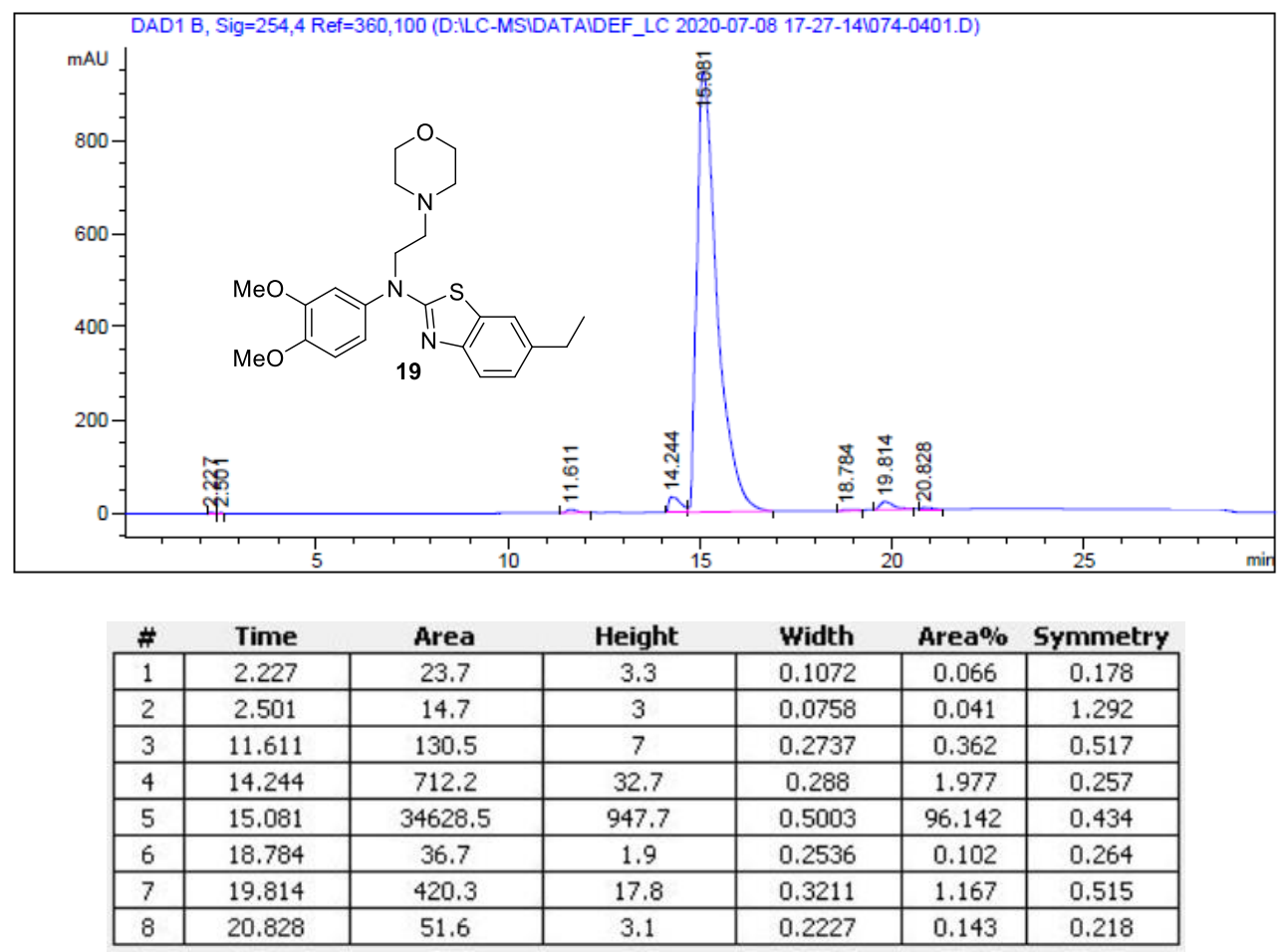

$N$-(3,4-Dimethoxyphenyl)-6-ethyl- $N$-(3-morpholinopropyl)benzo[d] thiazol-2-amine (20)

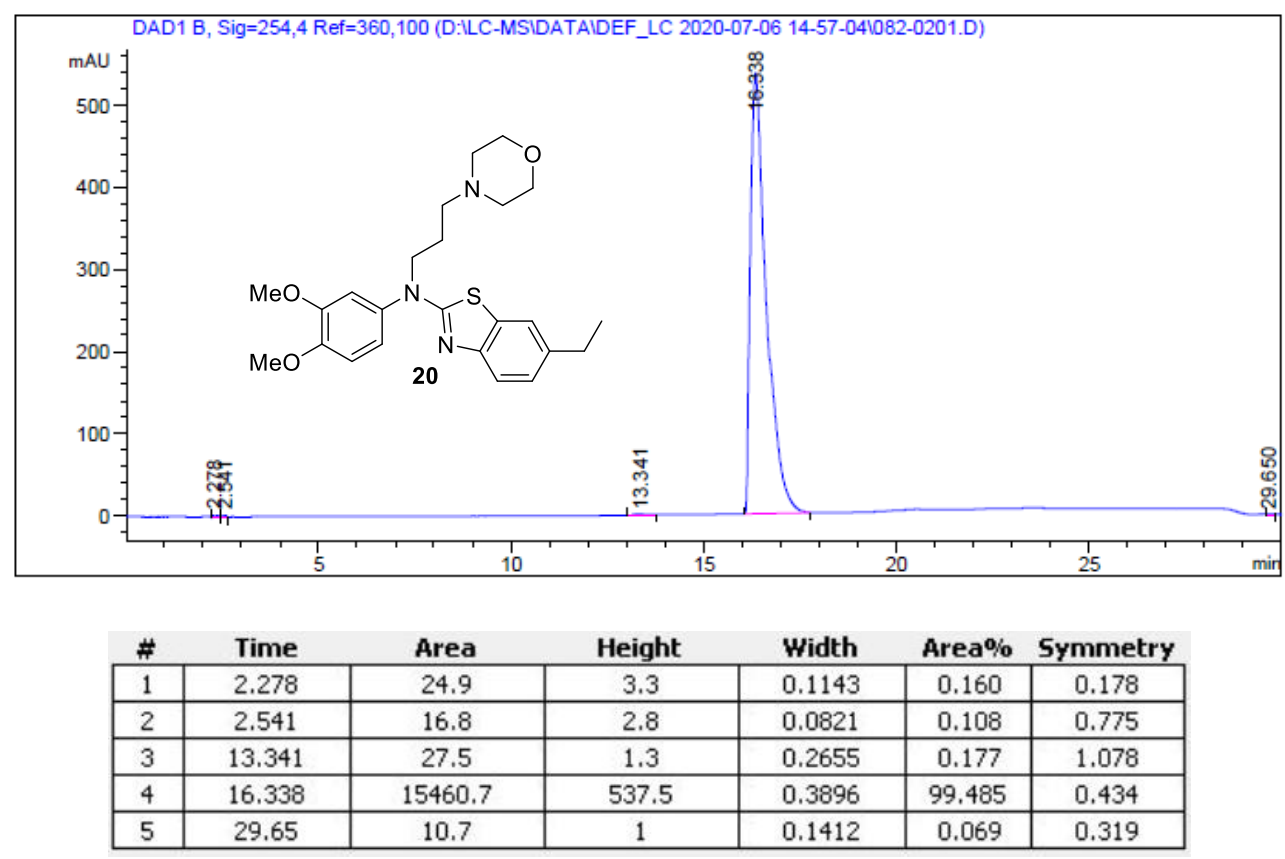


$N$-(3,4-Dimethoxyphenyl)-6-ethyl- $N$-(4-morpholinobutyl)benzo[d] thiazol-2-amine (21)

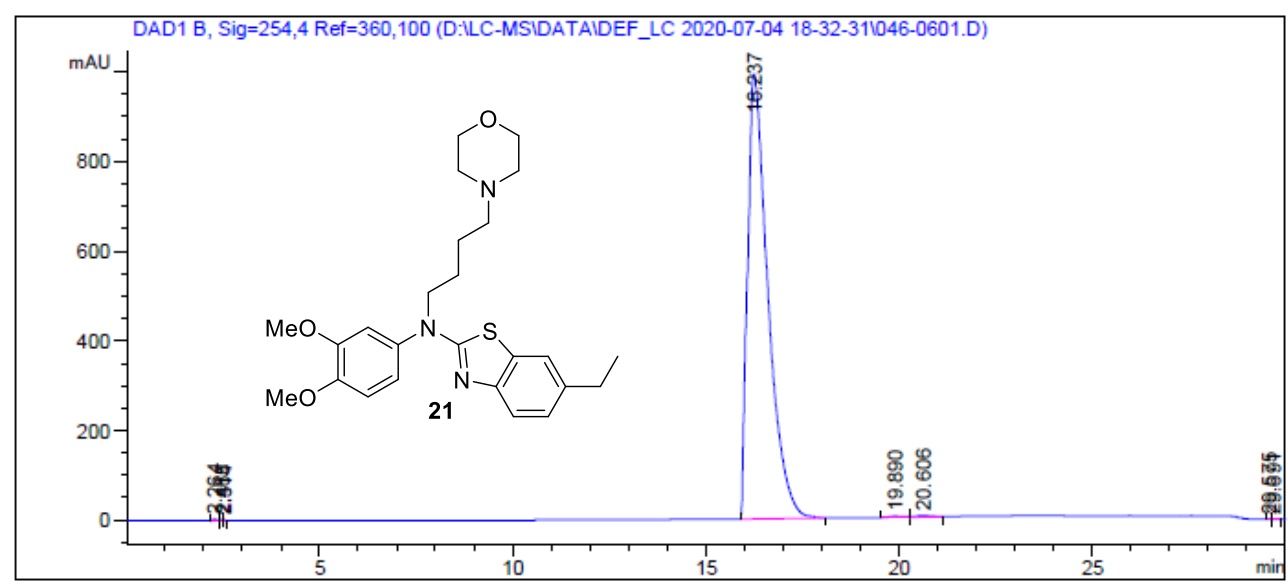

\begin{tabular}{|c|c|c|c|c|c|c|}
\multicolumn{1}{l}{ \# } & \multicolumn{1}{c}{ Time } & \multicolumn{1}{c}{ Area } & Height & \multicolumn{1}{c}{ Width } & \multicolumn{1}{c}{ Area\% } & \multicolumn{1}{c}{ Symmetry } \\
\hline 1 & 2.264 & 24 & 3.4 & 0.0989 & 0.066 & 0.542 \\
\hline 2 & 2.465 & 12.5 & 2.8 & 0.0631 & 0.034 & 1.611 \\
\hline 3 & 2.514 & 7.3 & 2.4 & 0.0466 & 0.020 & 0.506 \\
\hline 4 & 16.237 & 36235.5 & 992.2 & 0.5101 & 99.462 & 0.417 \\
\hline 5 & 19.89 & 54.1 & 2.4 & 0.2885 & 0.149 & 0.735 \\
\hline 6 & 20.606 & 80.1 & 3.5 & 0.3443 & 0.220 & 0.792 \\
\hline 7 & 29.575 & 5.5 & 1.1 & 0.0774 & 0.015 & 1.025 \\
\hline 8 & 29.691 & 12.6 & 1 & 0.1627 & 0.034 & 0.304 \\
\hline
\end{tabular}

$N$-(3,4-Dimethoxyphenyl)-6-ethyl- $N$-(5-morpholinopentyl)benzo[ $d]$ thiazol-2-amine (22)

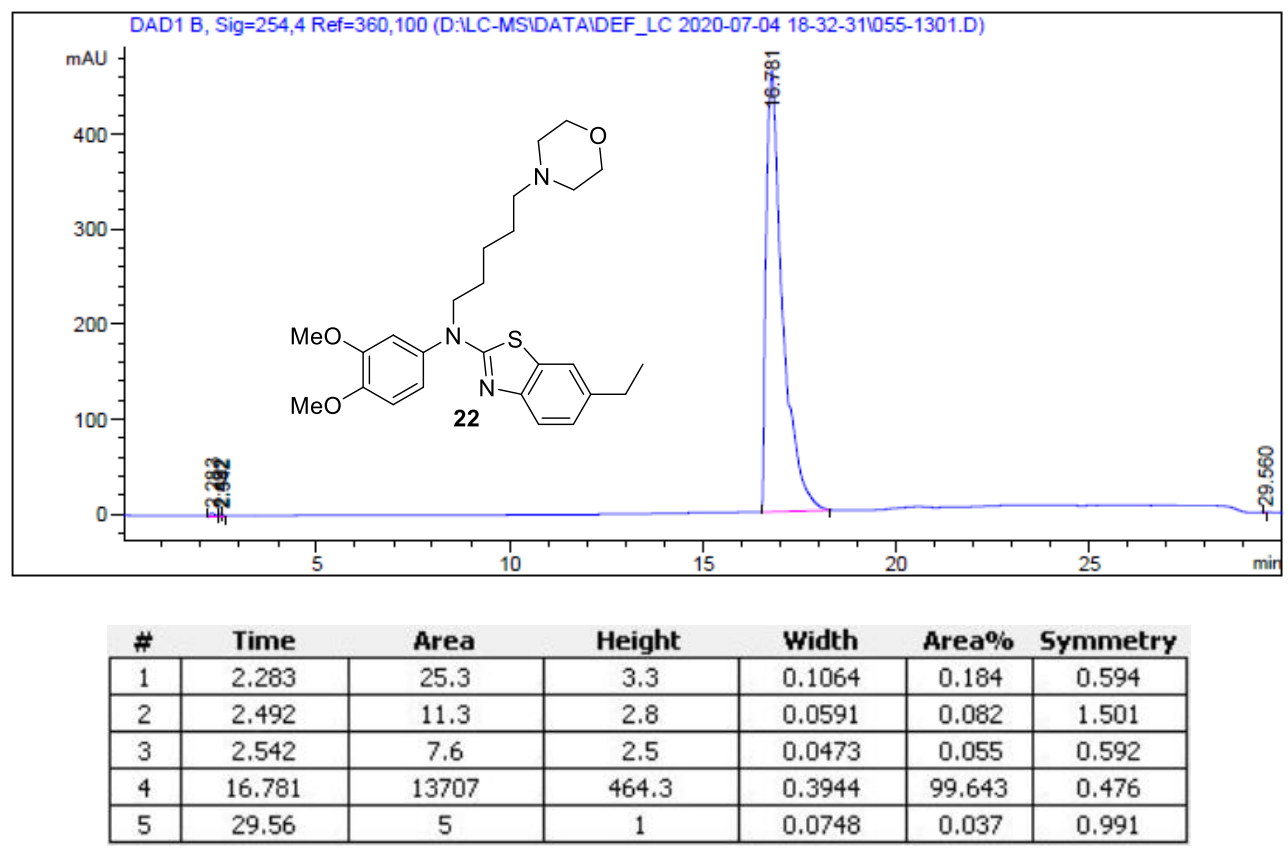


$N^{1}$-(3,4-Dimethoxyphenyl)- $N^{2}, N^{2}$-diethyl- $N^{1}$-(6-ethylbenzo[d] thiazol-2-yl)ethane-1,2-diamine (23)

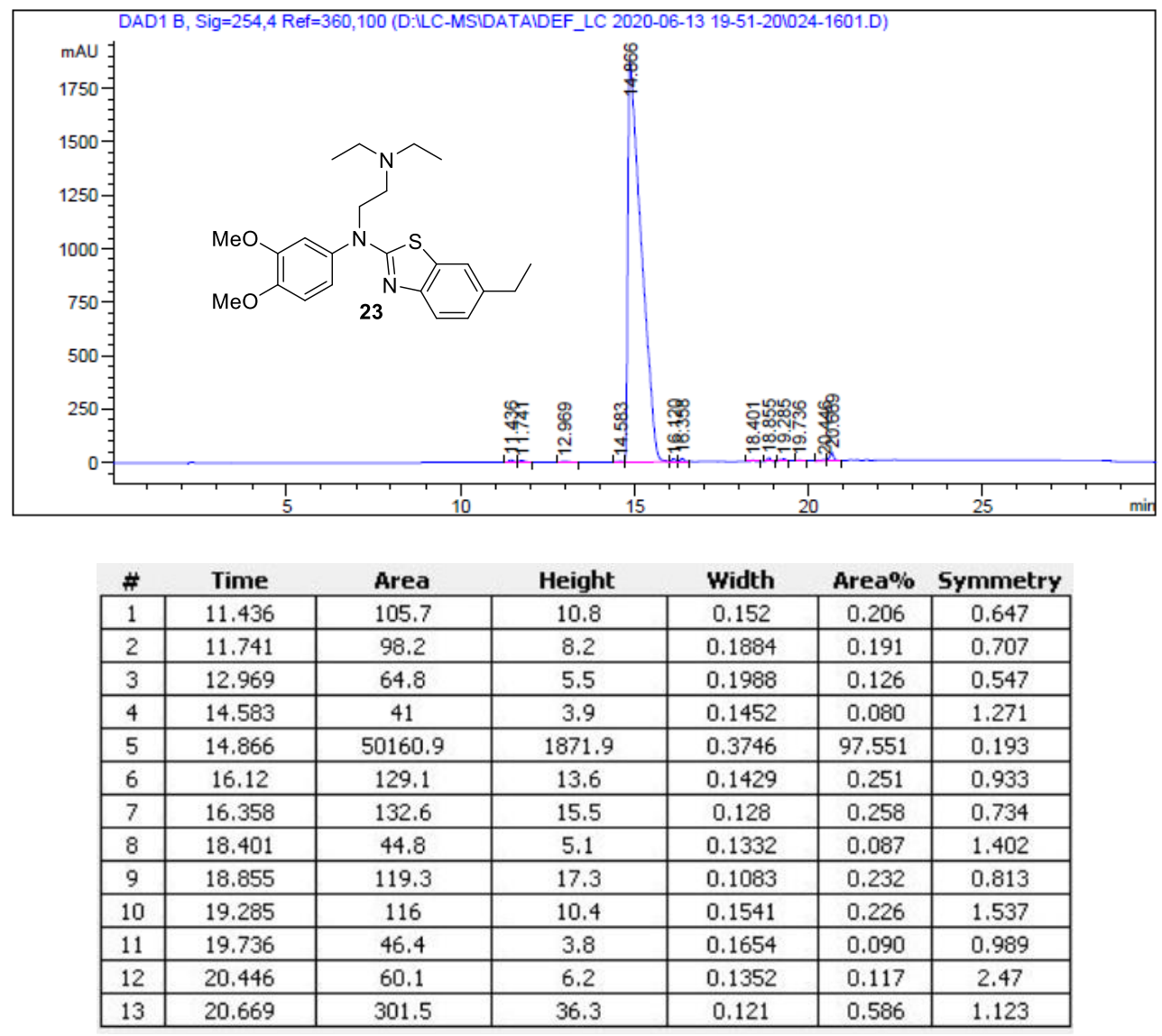

$N^{1}$-(3,4-Dimethoxyphenyl)- $N^{3}, N^{3}$-diethyl- $N^{1}$-(6-ethylbenzo $[d]$ thiazol-2-yl)propane-1,3-diamine (24)

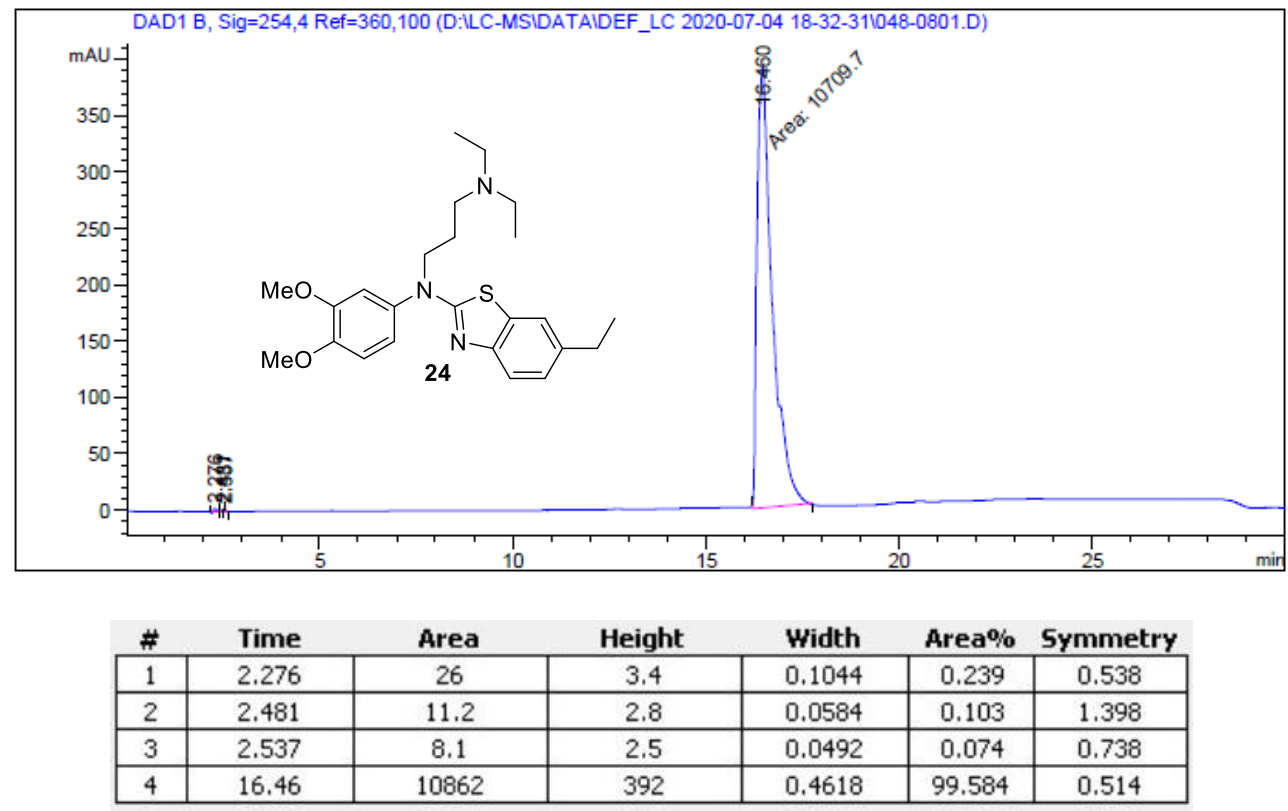


$N^{1}$-(3,4-Dimethoxyphenyl)- $N^{4}, N^{4}$-diethyl- $N^{1}$-(6-ethylbenzo $[d]$ thiazol-2-yl)butane-1,4-diamine (25)

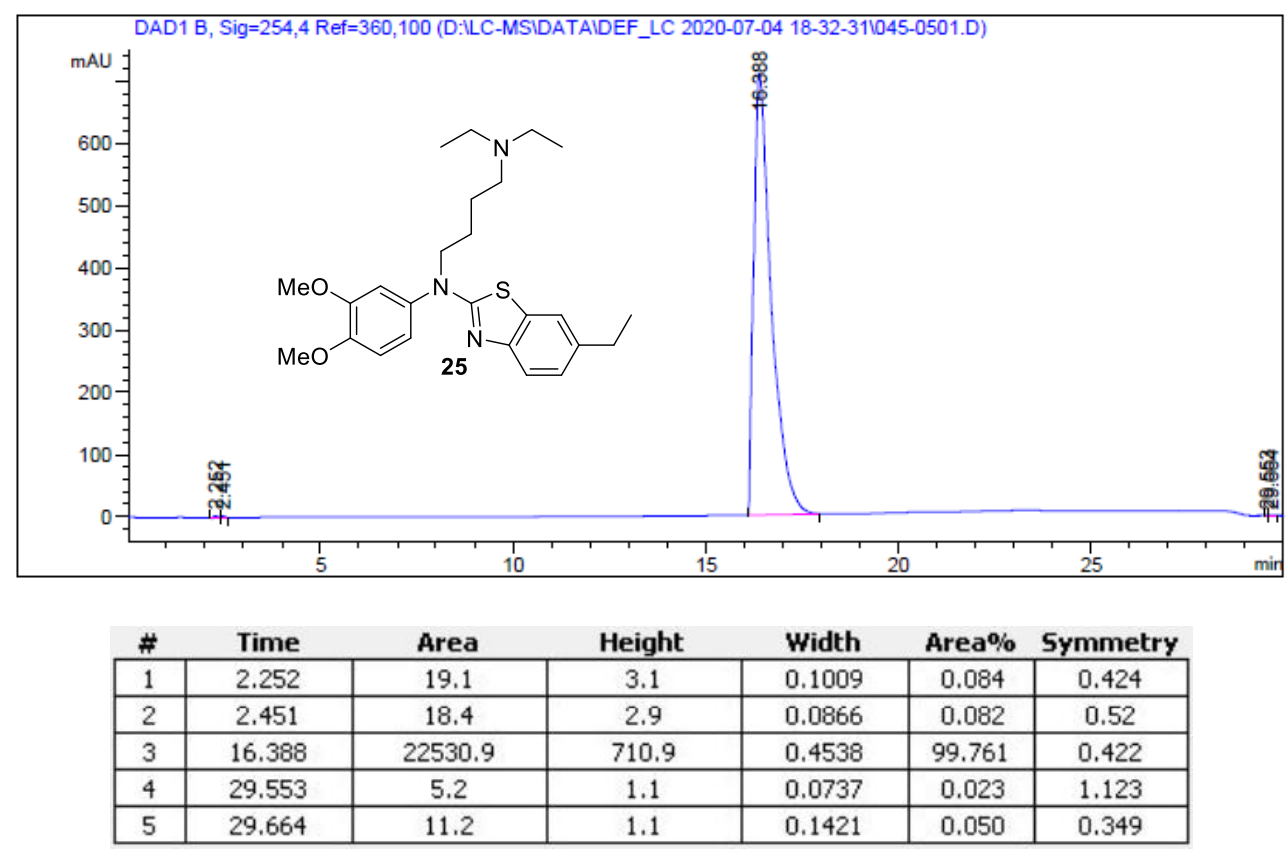

$N^{1}$-(3,4-Dimethoxyphenyl)- $N^{5}, N^{5}$-diethyl- $N^{1}$-(6-ethylbenzo $[d]$ thiazol-2-yl)pentane-1,5-diamine (26)

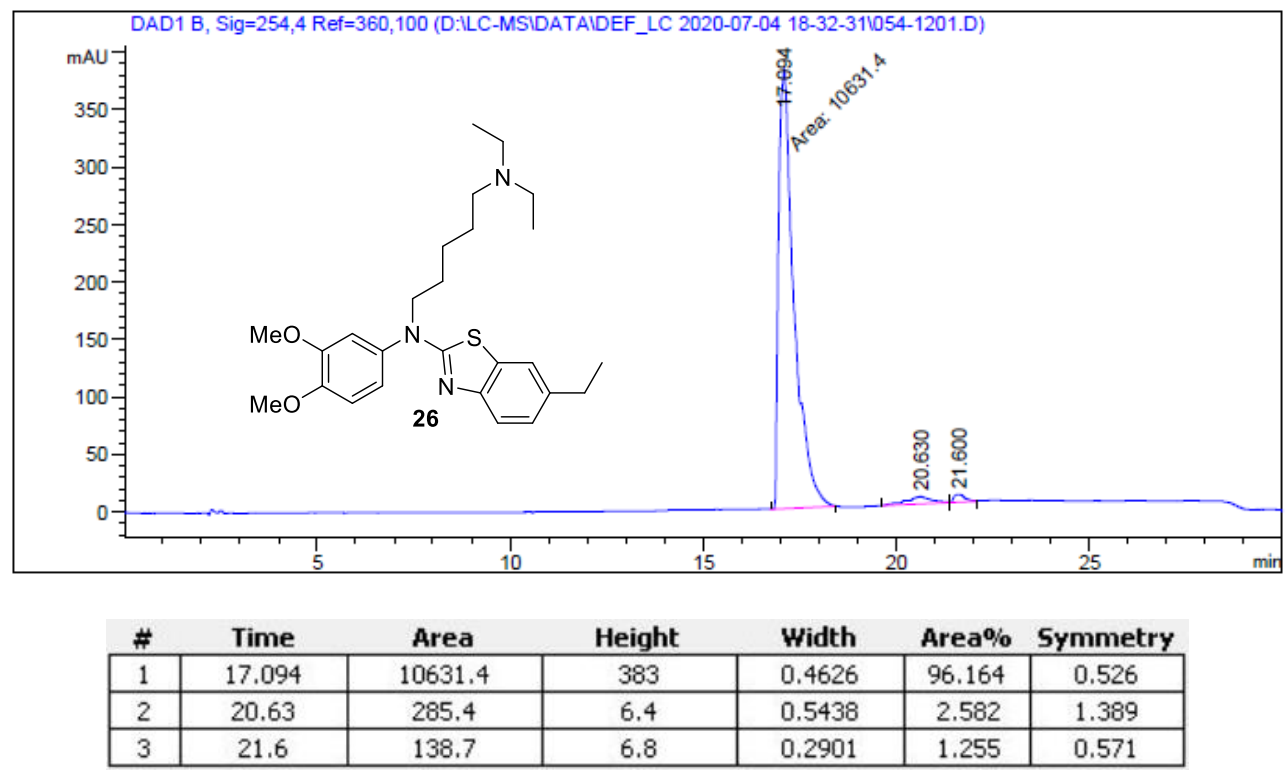


$N^{1}$-Cyclohexyl- $N^{2}$-(3,4-dimethoxyphenyl)- $N^{2}$-(6-ethylbenzo[d] thiazol-2-yl)ethane-1,2-diamine (27)

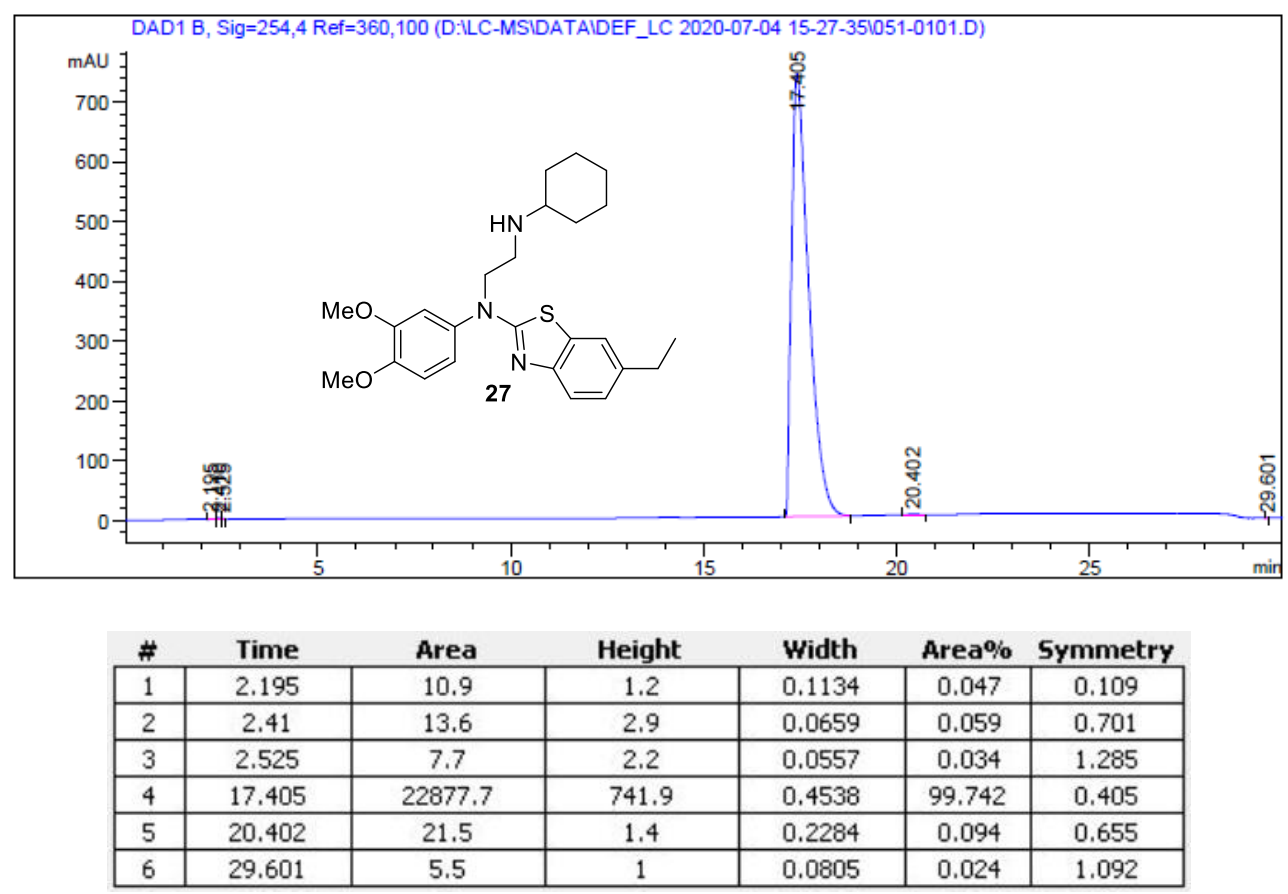

$N^{1}$-Cyclohexyl- $N^{3}$-(3,4-dimethoxyphenyl)- $N^{3}$-(6-ethylbenzo[d] thiazol-2-yl)propane-1,3-diamine (28)

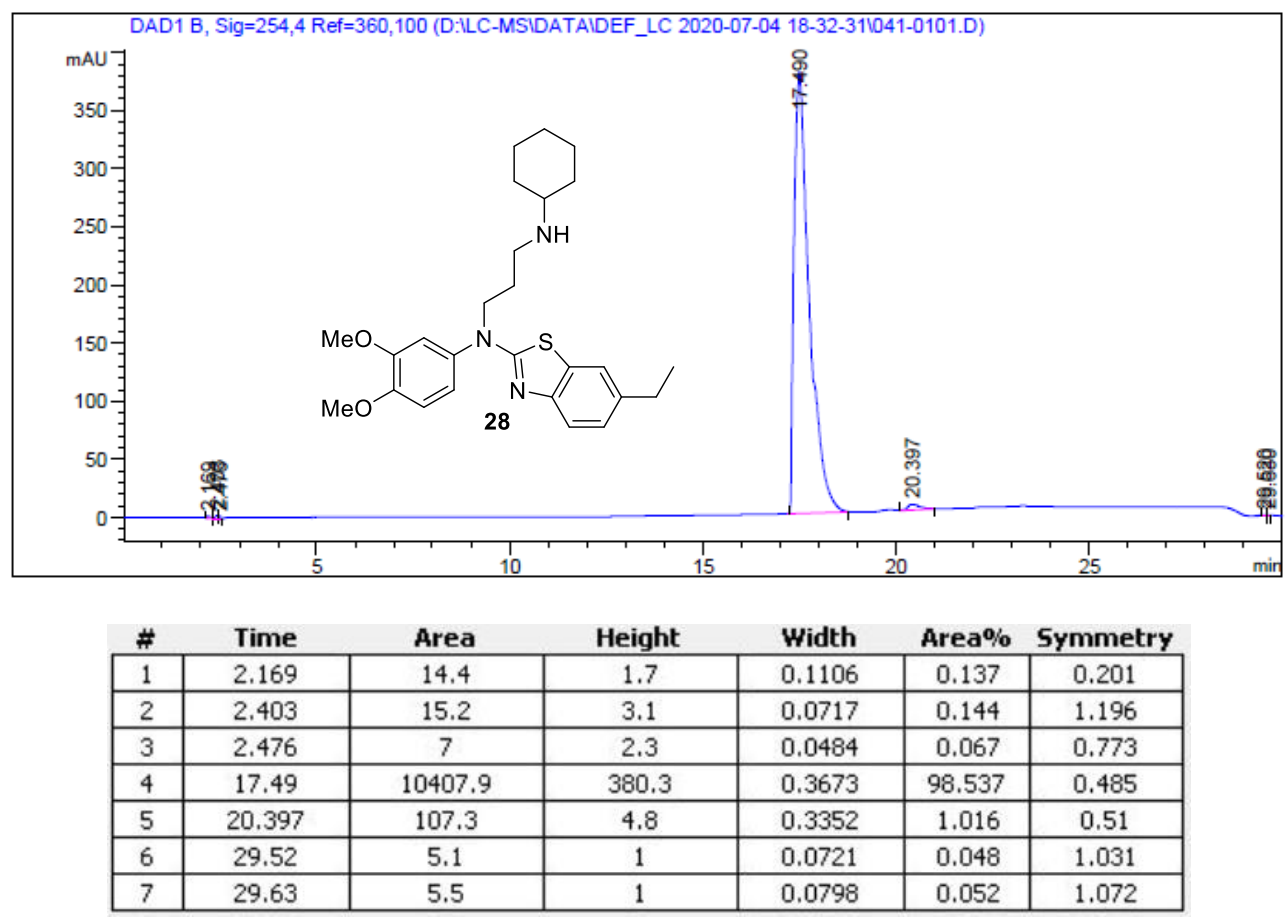


$N^{1}$-Cyclohexyl- $N^{4}$-(3,4-dimethoxyphenyl)- $N^{4}$-(6-ethylbenzo $[d]$ thiazol-2-yl)butane-1,4-diamine (29)

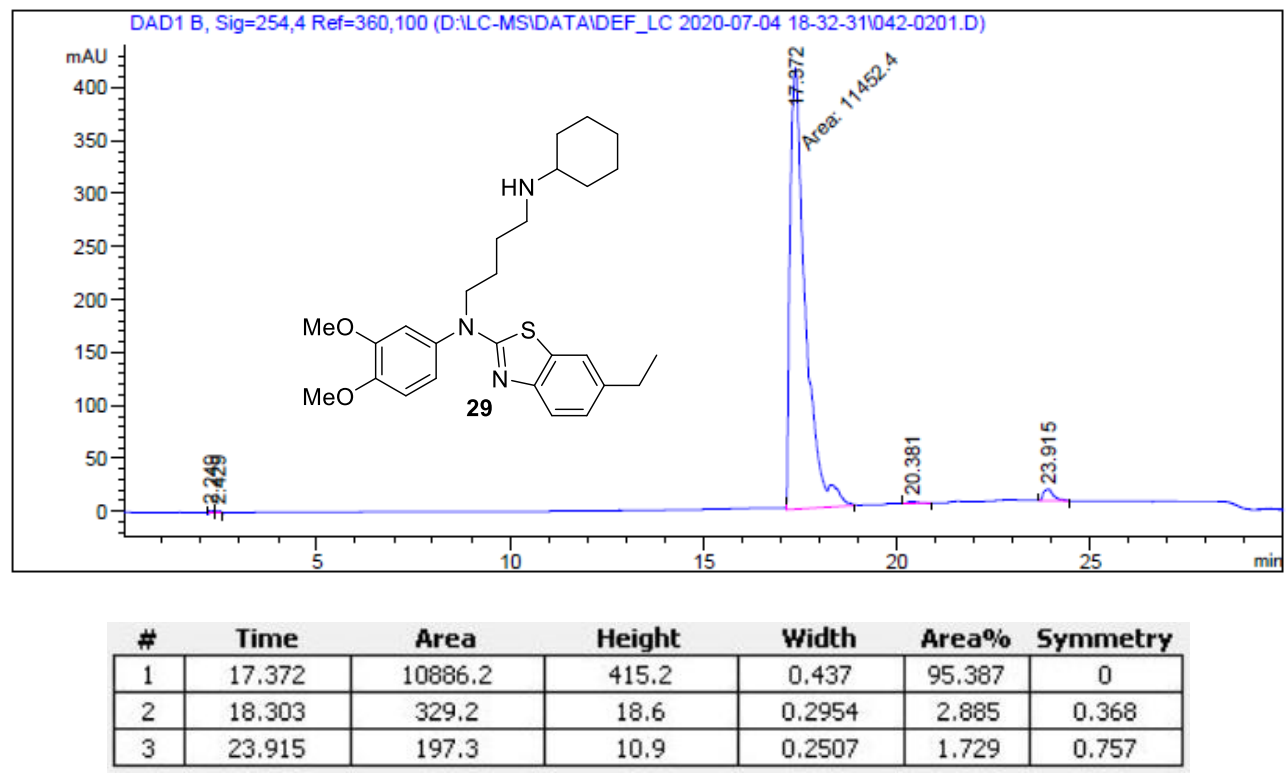

$N^{1}$-Cyclohexyl- $N^{5}$-(3,4-dimethoxyphenyl)- $N^{5}$-(6-ethylbenzo $[d]$ thiazol-2-yl)pentane-1,5-diamine (30)

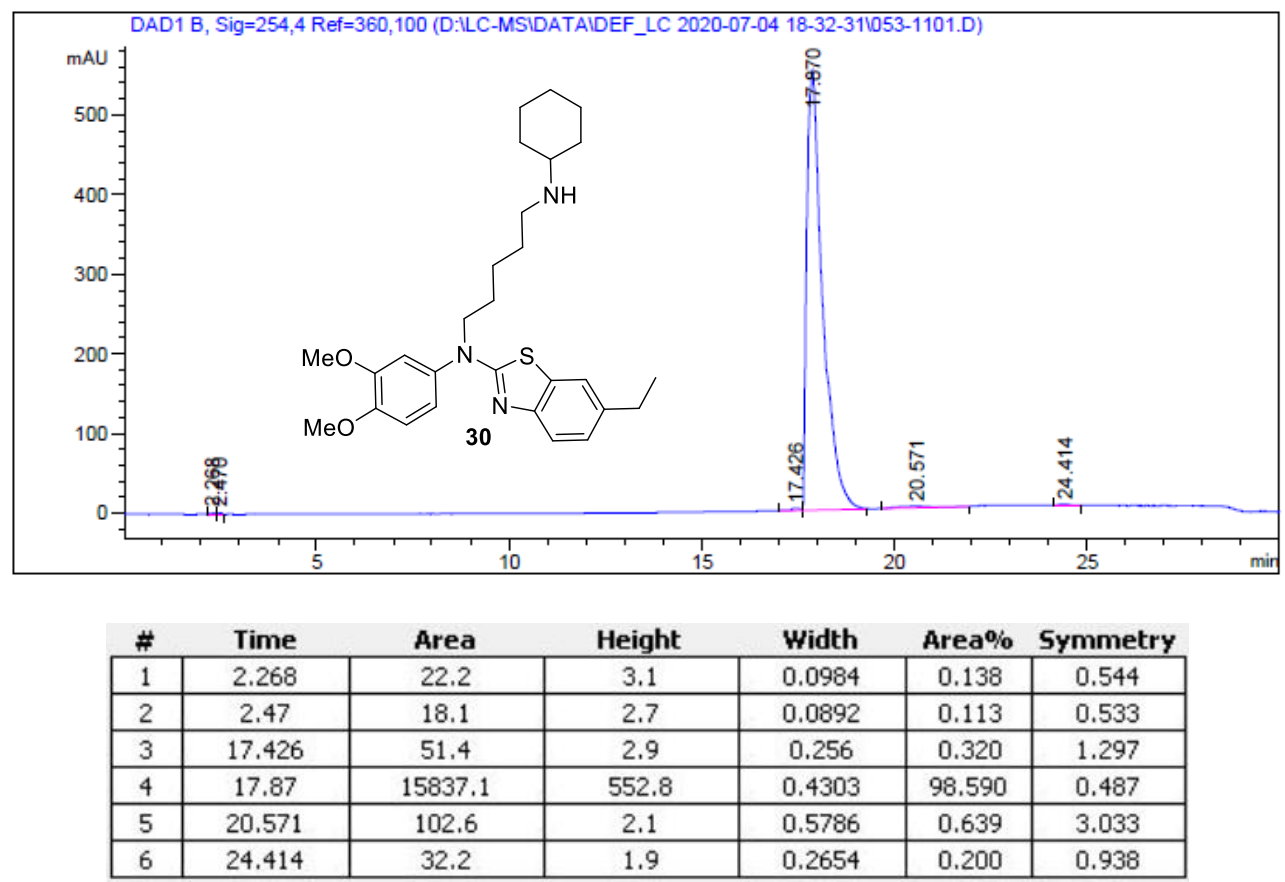


$N^{1}$-Cyclopentyl- $N^{2}$-(3,4-dimethoxyphenyl)- $N^{2}$-(6-ethylbenzo[d] thiazol-2-yl)ethane-1,2-diamine (31)

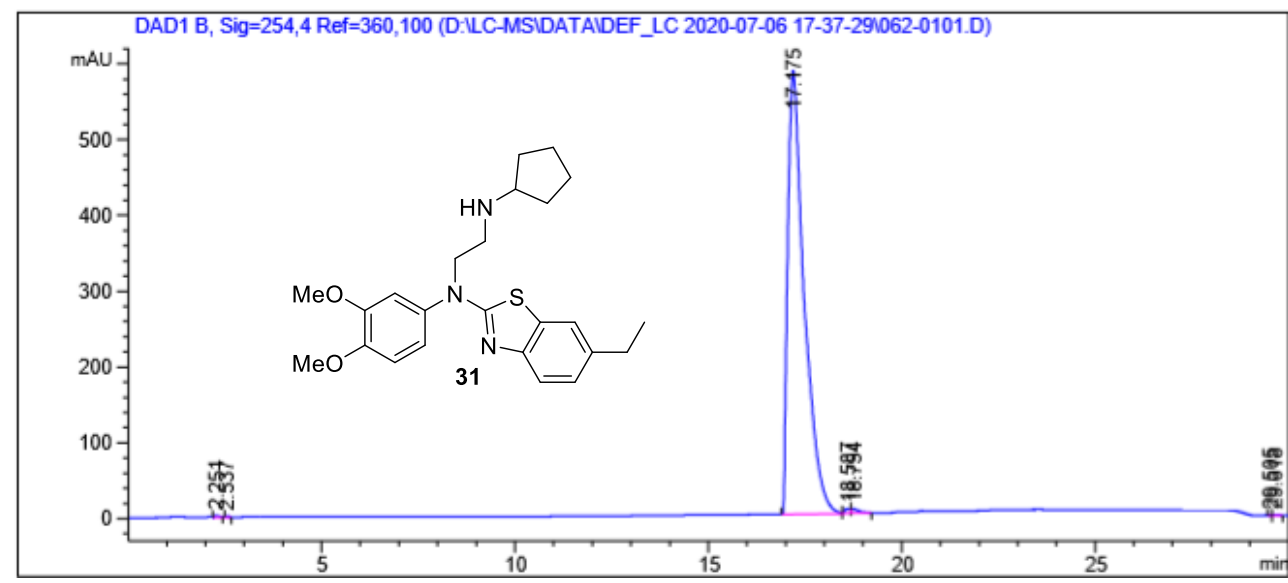

\begin{tabular}{|c|c|c|c|c|c|c|}
\hline $\begin{array}{c}\text { Peak } \\
\vdots\end{array}$ & $\begin{array}{c}\text { RetTime } \\
\text { [min] }\end{array}$ & Type & $\begin{array}{l}\text { Width } \\
\text { [min] }\end{array}$ & $\begin{array}{c}\text { Area } \\
{\left[\mathrm{mAU} \mathbf{U}^{*} \mathrm{~s}\right]}\end{array}$ & $\begin{array}{l}\text { Height } \\
\text { [mAU] }\end{array}$ & $\begin{array}{c}\text { Area } \\
*\end{array}$ \\
\hline & & & 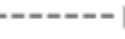 & $----n---n$ & ------ & $-0-$ \\
\hline 1 & 2.251 & BB & 0.1097 & 20.03531 & 2.66661 & 0.1152 \\
\hline 2 & 2.537 & BV & 0.0953 & 14.38633 & 2.01369 & 0.0827 \\
\hline 3 & 17.175 & BV & 0.4028 & $1.72052 \mathrm{e} 4$ & 585.60480 & 98.9026 \\
\hline 4 & 18.587 & VV & 0.1488 & 55.57937 & 5.74999 & 0.3195 \\
\hline 5 & 18.754 & VB & 0.1867 & 81.89280 & 5.73038 & 0.4708 \\
\hline 6 & 29.505 & VV & 0.0714 & 5.12052 & 1.07743 & 0.0294 \\
\hline 7 & 29.618 & vV & 0.1688 & 13.88304 & 1.08868 & 0.0798 \\
\hline
\end{tabular}


$N^{1}$-Cyclopentyl- $N^{3}$-(3,4-dimethoxyphenyl)- $N^{3}$-(6-ethylbenzo[d] thiazol-2-yl)propane-1,3-diamine (32)

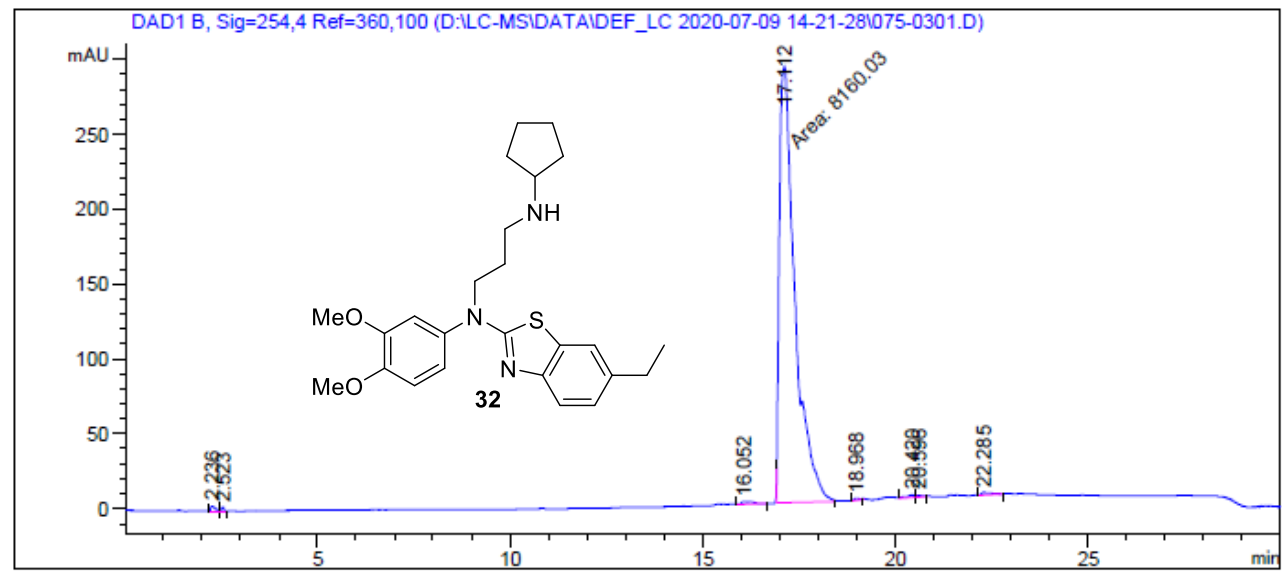

\begin{tabular}{|c|c|c|c|c|c|c|}
\hline$\#$ & Time & Area & Height & Width & Area\% & Symmetry \\
\hline 1 & 2.236 & 34.6 & 3.9 & 0.1263 & 0.409 & 0.244 \\
\hline 2 & 2.523 & 15.3 & 3.1 & 0.075 & 0.181 & 1.142 \\
\hline 3 & 16.052 & 42.6 & 2 & 0.2737 & 0.503 & 0.311 \\
\hline 4 & 17.112 & 8293.8 & 293.1 & 0.4717 & 97.936 & 0.451 \\
\hline 5 & 18.968 & 13.2 & 1.3 & 0.1437 & 0.156 & 0.383 \\
\hline 6 & 20.42 & 27.5 & 1.7 & 0.2241 & 0.325 & 2.322 \\
\hline 7 & 20.596 & 12.6 & 1.1 & 0.1573 & 0.149 & 0.519 \\
\hline 8 & 22.285 & 28.9 & 1.7 & 0.2304 & 0.342 & 0.269 \\
\hline
\end{tabular}

$N^{1}$-Cyclopentyl- $N^{4}$-(3,4-dimethoxyphenyl)- $N^{4}$-(6-ethylbenzo $[d]$ thiazol-2-yl)butane-1,4-diamine (33)

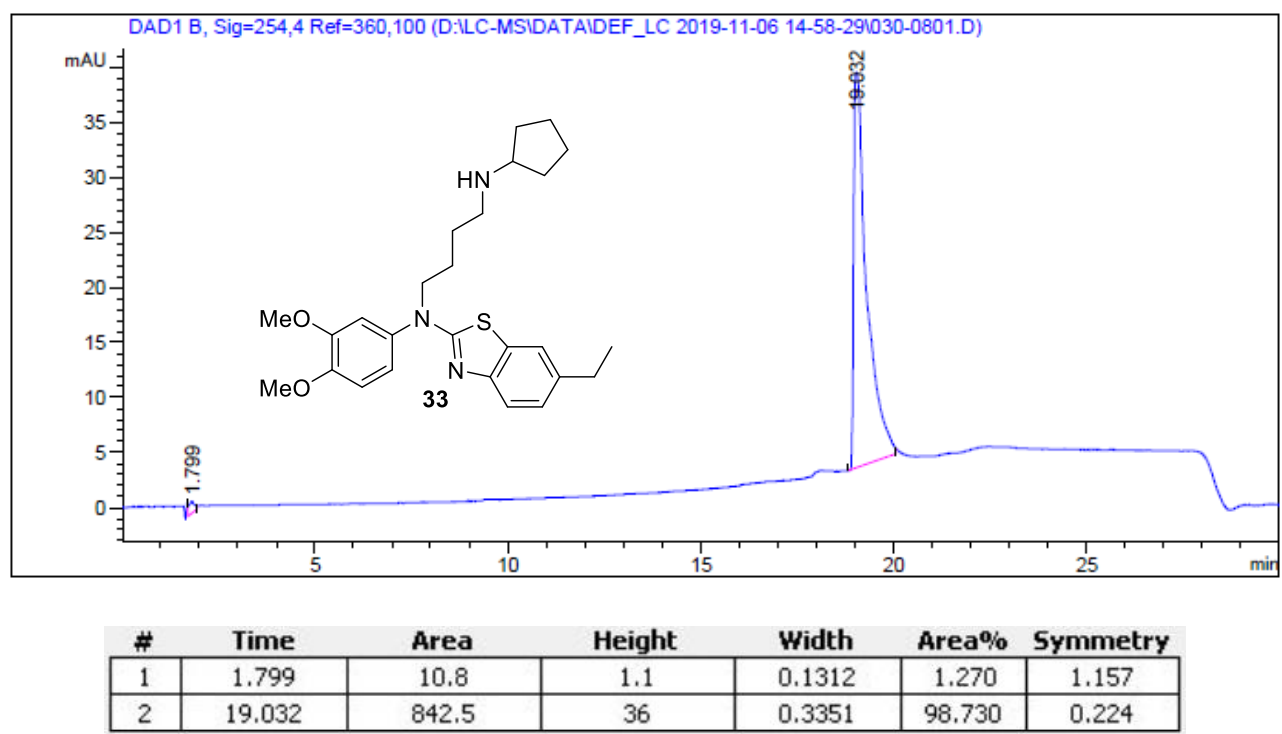


$N^{1}$-Cyclopentyl- $N^{5}$-(3,4-dimethoxyphenyl)- $N^{5}$-(6-ethylbenzo[d] thiazol-2-yl)pentane-1,5-diamine (34)

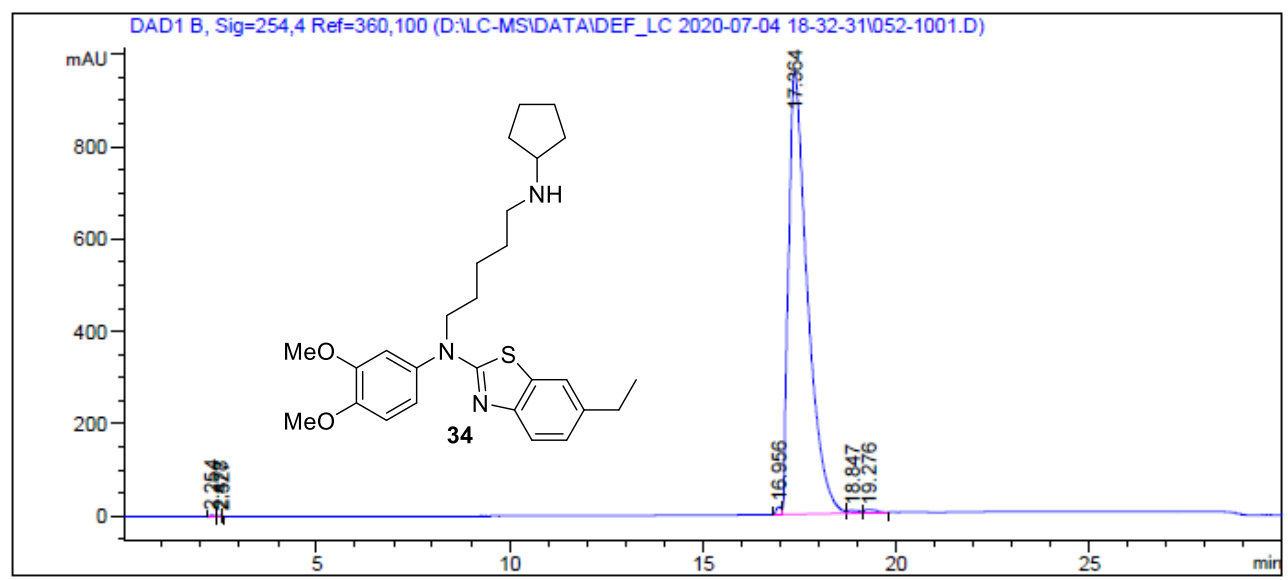

\begin{tabular}{|c|c|c|c|c|c|c|}
\multicolumn{1}{|c}{} & \multicolumn{1}{c}{ Time } & Area & Height & Width & \multicolumn{1}{c|}{ Area\% } & Symmetry \\
\hline 1 & 2.254 & 33.6 & 4.2 & 0.1057 & 0.102 & 0.308 \\
\hline 2 & 2.478 & 14.2 & 3.1 & 0.0649 & 0.043 & 1.781 \\
\hline 3 & 2.527 & 8.7 & 2.8 & 0.0487 & 0.026 & 0.506 \\
\hline 4 & 16.956 & 143.3 & 16.1 & 0.138 & 0.436 & 1.071 \\
\hline 5 & 17.364 & 32404.1 & 960.6 & 0.4976 & 98.611 & 0.421 \\
\hline 6 & 18.847 & 113.7 & 5.7 & 0.268 & 0.346 & 0.485 \\
\hline 7 & 19.276 & 143.1 & 7.3 & 0.2677 & 0.436 & 0.462 \\
\hline
\end{tabular}




\section{References}

1 Hirose, W.; Sato, K.; Matsuda, A. Fluorescence Properties of 5- (5,6-Dimethoxybenzothiazol-2yl)-2'-deoxyuridine (dbtU) and Oligodeoxyribonucleotides Containing dbtU. Eur. J. Org. Chem. 2011, 6206-6217.

2 Pfaendler, H. R.; Weimar, V. Synthesis of racemic ethanolamine plasmalogen. Synthesis 1996, $1345-1349$. 